\title{
ISSN 2367-7570
}

\section{Workshop \\ "Solar Influences on the Magnetosphere, Ionosphere and Atmosphere"}
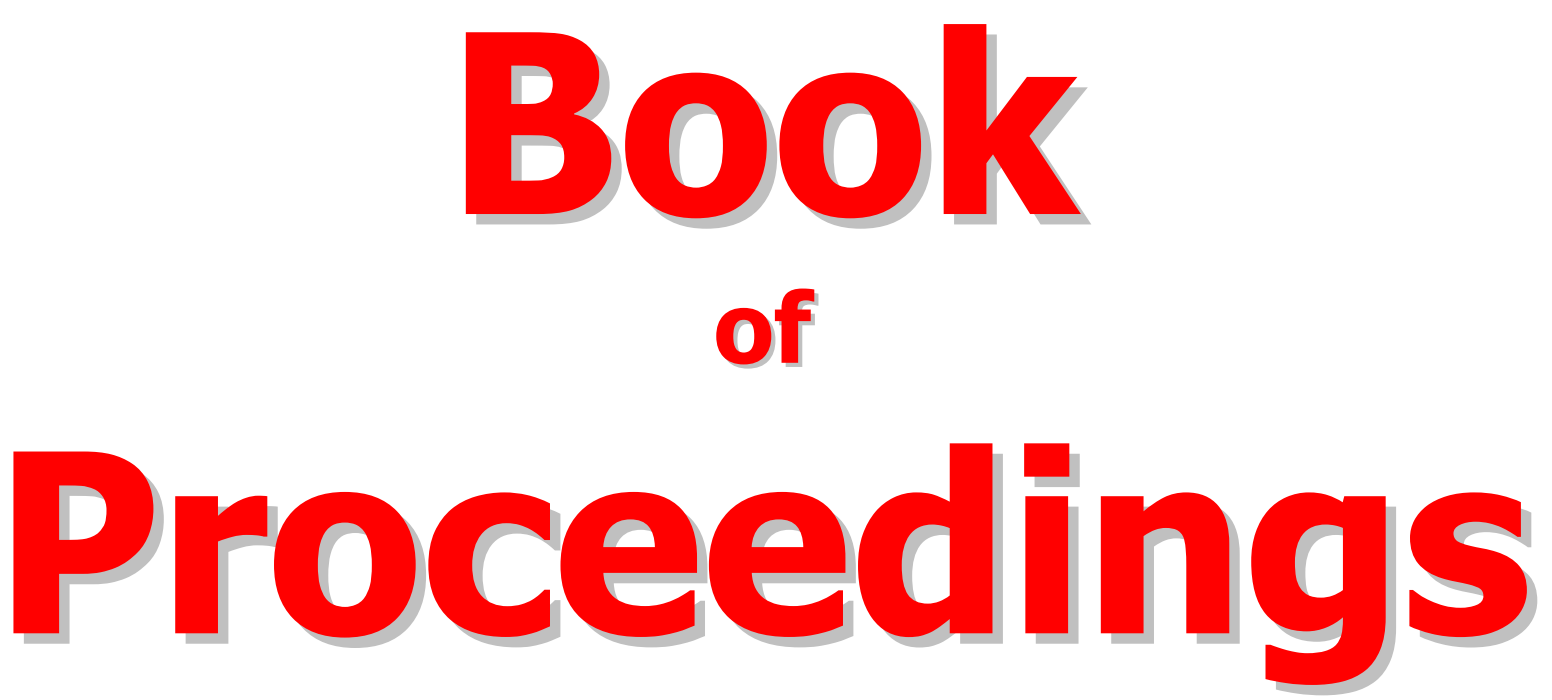

Twelfth Workshop

September, 2020 


\section{Organized by: \\ Space Research and Technologies Institute Bulgarian Academy of Sciences}

\section{Scientific Organizing Committee}

Katya Georgieva (Space Research and Technologies Institute, Bulgaria) - Chair

Crisan Demetrescu (Institute of Geodynamics, Romanian Academy)

Ana G. Elias (Universidad Nacional de Tucuman, Argentina)

Petra Koucká-Knižová (Institute of Atmospheric Physics, Czech Republic)

Olga Malandraki (IAASARS, National Observatory of Athens, Greece)

Dibyendu Nandi (Indian Institute for Science Education and Research, Kolkata, India)

Vladimir Obridko (IZMIRAN, Moscow, Russian Federation)

Atila Özgüc (Kandilli Observatory, Turkey)

Dragan Roša (Zagreb Astrronomical Observatory, Croatia)

Jean-Pierre Rozelot (Université de la Côte d'Azur, Grasse, France)

\section{Editors: Katya Georgieva, Boian Kirov, Dimitar Danov}

\section{DOI: 10.31401/WS.2020.proc}




\section{CONTENT}

\section{$\underline{\text { Sun and Solar Activity }}$}

Rozelot J.P., Kosovichev A.G., Kilcik A., Sahin S., Xu Y., Javaraiah J., Georgieva K., $\ddot{O}_{z g u ̈ c}$ A. Solar Multipolar Moments: an up-to-date Pedagogical Approach. Implications for Stellar Properties. Part I: an Analytical Overview (TUTORIAL)

Katsova M.M., Obridko V.N., Sokoloff D.D., Livshits I.M. On Superflares on the Sun and Stars

Koleva K., Chandra R., Joshi R., Devi P., Duchlev P., Dechev M. Morphological and Kinematical Study of Causally- Linked Filament Eruptions

Tsvetkov Ts., Petrov N. New 30-cm Solar Telescope at National Astronomical Observatory Rozhen

Tsvetkov Ts., Petrov N., Miteva R., Ivanov E., Popov V. Bulgarian Expedition for the Total Solar Eclipse on 2020 December 14

\section{Solar Wind-Magnetosphere-Ionosphere Interactions}

Bojilova R., Mukhtarov P. Functional Dependence of the Critical Frequency of the

Ionospheric F2-layer on Geomagnetic Activity

Bojilova R., Mukhtarov P. Relationship Between Short-Term Variations in Solar

Activity and Critical Frequency of the Ionospheric F2 Layer

Despirak I.V., Lubchich A.A., Kleimenova N.G. Certain Space Weather Conditions

for a Supersubstorm Appearance

Gromova L.I., Kleimenova N.G., Gromov S.V., Malysheva L.M. Local and Global

Longitudinal Geomagnetic SC Effects at High-Latitudes: Case Study of the

Interval of March 07-15, 2012

Guineva $V$., Werner R. Substorm Activity During the Geomagnetic Storm on 25

August 2018

Kleimenova N.G., Gromova L.I., Gromov S.V., Malysheva L.M. Strong High-Latitude

Geomagnetic Response to the Initial Phase of the Magnetic Storm on 5 April 2010

Lytvynenko O.A., Panishko S.K. Variations of the Ionosphere Scintillation

Parameters on the Observations of the Cosmic Radio Sources at the

Decameter Wave Range During Two Cycles of the Solar Activity

\section{Data Processing and Modeling}

Borisenko A.V., Podgorny I.M., Podgorny A.I. Using of Parallel Computing on Gpus

for MHD Modeling of Solar Flare Behavior in Real Time Scale

Shepherd, M.G., Shepherd, G.G., Barreto, C.S., Cho, Y.M., Chen, Y. Thermospheric

Wind Variability at High Latitudes - The Wind Wall

Podgorny A.I., Podgorny I.M., Borisenko A.V. Study of the Solar Flare Mechanism

by MHD Simulation in the Corona Above the Active Region in Real Time

Scale 


\section{Instrumentation for Space Weather Monitoring}

Dachev T., Semkova J., Tomov B., Matviichuk Y., Dimitrov P., Koleval R., Jordanova M., Bankov N., Litvak M., Mitrofanov I., Golovin D., Kozyrev A., Malakhov A., Mokrousov M., Sanin A., Tretyakov V., Fedosov F., Ploc O., Shurshakov V., Benghin V. A Comparison of the Neutron Dose Measurements Completed by the Bulgarian and Russian Instruments Outside the Zvezda Module of the ISS

Dachev T., Tomov B., Matviichuk Y., Dimitrov P., Semkova J., Jordanova M., Bankov N., Ploc O., Litvak M., Tretyakov V., Shurshakov V., Benghin V. Registration of Neutrons with a Single Detector Liulin Type Spectrometer

Kalinichenko M.M., Kuhai N.V., Konovalenko O.O., Bubnov I.M., Erin S.M.,

Romanchuk O.I. Giant Ukrainian Radio Telescope as an Instrument for Interplanetary Scintillation Observations

\section{Solar Influences on the Lower Atmosphere and Climate}

Chapanov Ya. Decadal NAO Oscillations Excited by TSI Cycles

Chapanov Ya., Ron C, Vondrák J. Solar Influence on Seismic Energy

Komitov B., Kaftan V. The Volcanic and Solar Activity Relationship During the

Last $\sim 460$ Years. Could a Significant Part of the "Sun-Climate" Relationship

Goes Through Lithosphere?

Komitov B., Kaftan V. The Beech Tree Ring Widths, Solar-Climatic Relationships and Solar Dynamo Regime Changes

Tonev P.T. Observations Supporting Hypothesis for Global Electrical Circuit as

Mediator between Solar Events and Weather

Veretenenko $S$. $V$. Effects of Solar Proton Events of January 2005 on Intensity of the Stratospheric Polar Vortex in the Northern Hemisphere

Belakhovsky V.B., Pilipenko V.A., Sakharov Ya.A., Selivanov V.N. The GIC growth in electric power lines during magnetic storm on 7-8 September 2017 


\section{Preface}

The Twelfth Workshop "Solar Influences on the Magnetosphere, Ionosphere and Atmosphere" was scheduled to be held from June 3 to 7, 2020 in Primorsko, Bulgaria. Due to the coronavirus pandemic, the Local Organizing Committee decided to postpone the workshop until September. Unfortunately, in September the situation was not better, and it was not possible to hold a face-to-face meeting. Therefore, the workshop was held online. 24 papers with a total of 76 authors from 11 countries are included in these Proceedings.

The Scientific Organizing Committee and the Editors of the Proceedings thank all the participants in the Workshop and contributors to the Proceedings. 


\title{
Solar Multipolar Moments: an up-to-date Pedagogical Approach. Implications for Stellar Properties. Part I: an Analytical Overview (TUTORIAL)
}

\author{
Rozelot $^{1}$ J.P., Kosovichev ${ }^{2}$ A.G., Kilcik ${ }^{3}$ A., Sahin ${ }^{3,4}$ S., Xu ${ }^{5}$ Y., Javaraiah ${ }^{6}$ J., Georgieva ${ }^{7}$ K., \\ $\ddot{O} z g \ddot{u} c^{8} A$. \\ ${ }^{1}$ Universit'e de la Cote d'Azur, Grasse, France \\ ${ }^{2}$ New Jersey Institute of Technology, USA \\ ${ }^{3}$ Akdeniz University, Antalya, Turkey \\ ${ }^{4}$ Northumbria University, UK \\ ${ }^{5}$ Shandong University, Wuhei, China \\ ${ }^{6}$ Retired from Indian Astrophysical Institute, Bangalore, India \\ ${ }^{7}$ BAS, Sofia, Bulgaria \\ ${ }^{8}$ Kandilli Observatory, Istanbul, Turkey \\ e-mail: jean-pierre.rozelot@univ-cotedazur.fr \\ jp.rozelot@orange.fr
}

\begin{abstract}
.
Solar gravitational multipolar moments have not been yet extensively analyzed. However, they are at the crossroads of solar physics, solar astrometry, celestial mechanics and General Relativity. Their values reflect the physics of solar models: non-rigid rotation, solar latitudinal rotation, solar- core properties, solar-cycle variations and structure evolution. Their temporal variations are still often neglected; they are yet an essential aspect for constraining solar-cycle modeling or solar-evolution theories. They induced planet-planet inclinations in multitransiting systems gravitating in the neighboring of a star, leading to key issues future studies. This paper is devoted to an analytical analysis; a second part will address an helioseismology analysis.

\section{Introduction}

It has been suggested by Dicke (1974) that the measured excess of solar oblateness over the oblateness due to the surface rotation alone might be due to the existence of solar gravitational moments, themselves inter alia, due to a rapidly solar core. This implies the knowledge of the mass distribution inside the Sun, and to first order, the gravitational quadrupole moment. The question is not completely solved and remains of great astrophysical interest, if, for example, the core is a fossil remnant of the rotation of the young Sun. The surface rotation of young solar-type stars, slightly more massive that the Sun decreases with age. If the surface rotation decreases, the central question is why a rapidly rotating core is still left in the solar/stellar interior? Another issue concerns the inclination of the planets inside our solar system, directly linked with the solar quadrupole moment. This point has been generalized for exoplanets, to calculate the mutual inclination induced between two (or more) planets as a function of the stellar oblateness (Spalding and Batygin, 2016).

Despite several attempts, we are still ignoring the exact determination of the successive solar gravitational moments, usually designed by $J_{n}$. By carefully analyzing in the existing literature the different techniques and models aiming at detecting $J_{n}$ for $n=2$, Pireaux and Rozelot (2003) assign $J_{2}$ to be $\approx(2.0 \pm 0.4) \times 10-7$, bearing in mind that $J_{2}$ is constraint by the lunar librations to be less than 3.10-6 [Rozelot and Bois, 1997]. Higher moments $(n>2)$ are not known with enough accuracy to be reasonably used today in modeling the solar interior
\end{abstract}


properties. Furthermore, we do not know their temporal dependence, even if some attempts have already been made. They are notoriously difficult to measure directly: detecting a so faint value is at the cutting edge of the current available techniques, even through space dedicated missions. Helioseismology may provide an indirect alternative, that indeed has been used. However, the analysis to infer their estimates requires the development of efficient inversion methods and algorithms. By contrast, the Sun's interior structure and dynamics affect our solar planets trajectories, Mercury at first. Confrontation of collected data from spacecraft orbit determination with computed ephemeris by various institutes, are well-suited to determine $J_{2}$ as a sub-product of the crossed analysis. It could be a mean to analyze their temporal variations, as today long-time series and space series of data are available.

To sum up, gravitational moments of a body in rotation are of fundamental interest, being at the crossroad of celestial mechanics, solar physics, solar astronomy-astrometry and General Relativity. Their accurate determinations, both theoretically and from observations lead to a better knowledge of the internal properties of the star, down to the core. By their external influences, they constrain planet-planet inclinations in multi-transiting systems gravitating in the neighboring of the rotating body -a forward thinking issue.

\section{Effects of rotation upon the internal structure of a self-gravitating body.}

Let us consider a single star that rotates along a fixed direction in space, with an angular velocity $\omega$. The question we are faced is to determine its external shape. First assume that, for $\omega=0$, the star is a gaseous body in gravitational equilibrium. In tridimensional space, for such a fluid mass at rest, it has been demonstrated that the sphere is the unique solution to the problem of hydrostatic equilibrium. The issue complicates when the initial (perfect) spherical body is set rotating at an angular velocity $\omega$. Axial rotation modifies the shape equilibrium configuration by adding a centrifugal acceleration term, breaking the spherical symmetry. The problem is then to determine the outer shape of the star, which is more complex than a simple ellipsoid, -then called spheroid- due to its stratification in density and the non-uniform distribution of the velocity rates of the matter at different latitudes and in depth. As a result, the exact equilibrium shape will show distortions at the free boundary, characterized by shape coefficients $s_{n}$ (related to asphericities coefficients as we will see in paragraph 2.3.1). Assuming spherical symmetry, the free surface is thus described as

$$
r(\theta)=R_{s p}\left[1+\sum_{n=0} s_{2 i} P_{2 n}(\cos \theta)\right]
$$

where $r$ is the vector radius, $R_{s p}$ the radius of a sphere of equivalent volume of the body, $\theta$ the polar angle (colatitude); $P_{2 n}(\cos \theta)$ are even Legendre polynomials. Such a development of the radius of a rotating body is of interest as the photospheric radius is one of the fundamental parameters governing the radiative equilibrium of a star. Moreover, it is straightforward to see that the flateness $f$ (in the general case of a fluid in rotation) is a linear function of the asphericities terms: $f=-(3 / 2) s_{2}-(5 / 8) s_{4}-(21 / 16) s_{6}-(\ldots)$.

\subsection{Enhancing the historical diagnostic.}

Considering the Earth as a rotating ellipsoid in uniform rotation $\omega$, Newton gave in his "Philosophiae Naturalis Principia Mathematica" in 1687, an approximate formulation of the Earth's flattening $f$, as a function of surface gravity $g_{s}: f=5 / 4 \omega^{2} \times R_{e q} / g_{s}$, where $R_{e q}$ was the equatorial radius (of the body). Huyghens, in 1690, reformulated the flattening in the form $f=$ $5 / 4 \omega^{2} \times R_{e q} / g_{s}$, still commonly used as a first approximation.

Since then, the question of the flattening of rotating bodies has been tackled by several authors and by several means. The "classical theory" goes back with Maclaurin (1742) and Clairaut (1743) who treated the case of a uniform ellipsoid. Then Jacobi (1834), Riemann 
(1860) and Chandrasekhar $(1965,1966)$ processed the spheroids case, Poincar'e (1891) those of a uniform torus, and Jeans (1914) polytropic cylinders. Solutions obtained by these methods are consistent only if the distributions of density and angular velocity are restricted to certain special well determined laws. However, the method seems not limited to slow rotation, and can goes to more rapidly rotation, but only when the quantity $|T / W|$, ratio of the kinetic energy of rotation $(T)$ to the (negative) gravitational energy $(-W=|W|)$ may approaches 0.5 [Lyttleton, 1953] for the most flattened Maclaurin spheroids. A star is thus considered to be "slowly rotating" if $|\mathrm{T}|<<|\mathrm{W}|$, without regard to the equatorial velocity or the ratio of centrifugal force to gravity at the surface. "Slowly rotating" stars may have surface layers which are severely distorted by rotation, with consequences of the distribution of the emitted light [Ostriker and Mark, 1968]. Since then, an extensive literature on the equilibrium structure of rotating bodies has been produced which can be roughly divided into three main approaches.

- The first and older one introduces level surfaces to describe the limb shape of the rotating body. The problem is carried to successively higher approximations by transforming the expressions for the gravitational potential to level surfaces. An extension of this method has been made by Bruns (1878), Radau (1885), Darwin (1899), Wavre (1932), Milne (1923), Chandrasekhar (1933) or Chandrasekhar and Lebovitz (1962) who treated objects with equipotentials which deviate only slightly from oblate surfaces. Extensive solutions have been widely studied by Modolenvsky (1988), who reached to the conclusion that the physics of equilibrium figures (density $\rho$, gravity $g$, pressure $p$ ) are completely determined by the geometrical stratification of the rotating body (Modolenvsky calls "stratification" the geometry of the successive equilibrium surfaces of a rotating body when "looking" progressively from the surface to the interior). Important contributions were latter on conducted by Hubbard (1975), Zharkov (1978), Moritz (1980), and many others. This theory, completed with results obtained from the advent of artificial satellites, has been widely used in geophysics and is still used in specific cases, such as for planets, with an incredible accuracy (ex: $J_{2}$ Mars $=$ $1.860718 \times 10^{-3}$ according to Yuan et al. (2002), from a 75th degree and order model).

- A second method avoids the difficulties encountered in accurately determining the potential, by treating it as a given quantity. For instance, in the Roche model (1849 for his first paper), the potential is taken to be that of a point mass, an approach taken again more recently by Essen (2014) who deduced accurate analytical results for the Sun and planets connecting the oblateness, the gravitational quadrupole moment and the angular velocity parameters.

- The most straightforward approach has been first taken by James (1964) and Stoeckly (1965), who numerically integrate the partial differential equations of equilibrium. The method has no obvious limitations and can be made as precise as is desired, although an accurate representation of the equidensity surfaces requires a fairly dense grid of points and correspondingly lengthy computations. The difficult part of this problem -satisfying Poissons equation in the interior with boundary conditions on a surface determined by the solution of the equations of equilibrium - can be solved by successive approximations. Closer to us, Maeder (1999) discussing the von Zeipel theorem ${ }^{1}$ generalized to account for differential rotation in the case of a "shellular" rotation law (i.e. when $\omega$ is constant on isobaric surfaces) has found that this differential rotation increases the oblateness. Mathis et al. (2018) reviewed the stars equilibrium forms, of arbitrary structure, distorted by rotation and tides. Up-to-date rotating models have been produced, such as those produced by the ESTER code [Rieutord et al., 2016], in which structure and rotation are inherently coupled in two dimensions.

\footnotetext{
${ }^{1}$ This theorem states that any internal source of distortion in the gravitational field at the surface will manifest itself as a change of shape in the surface layers. Thus, measuring the shape of these is equivalent to measuring surfaces of constant gravitational potential.
} 


\subsection{The Maclaurin's approach}

The stability of the spheroidal figures of equilibrium of a rotating fluid was studied by many authors at the end of the 19th century [such as Dedekink in 1860, Jacobi in 1834 or Rieman in 1876]. Maclaurin first established in 1742 that ellipsoids are stable or unstable according to whether their eccentricities are less than or greater than 0.9528. However, Rumiansev (1959) using a rigorous definition of the stability of figures of equilibrium proved that ellipsoids of revolution remain stable ${ }^{2}$ as long as their eccentricities are less than 0.8126 .

Defining $q$ by

$$
q=\frac{3 \omega^{2}}{4 \pi \rho_{m} G}\left(=\frac{\omega^{2} R_{e q}^{2} R_{p o l}}{G M}\right),
$$

where $G$ is the gravitational constant, $\rho_{m}$ the mean density of the body and $M$ its mass (to first order, $\left.M=(4 / 3) \pi \rho R^{2}{ }_{e q} R_{p o l}\right)$, equations are closed by $q=\left(3 /\left(2 l^{3}\right)\right)\left[\left(3+l^{2}\right) \arctan l-3 l\right]$ where $l$ is the inverse of the second eccentricity of the ellipsoid $l=R_{p o l} /\left(\mathrm{R}^{2} \text { eq }-\mathrm{R}^{2}{ }_{\mathrm{pol}}\right)^{1 / 2}$ related to its first eccentricity by $\varepsilon=1 /\left(1+l^{2}\right)^{1 / 2}$. The above equation in $q$ has one root $l_{o}=0.717$ corresponding to an eccentricity of the body of $\varepsilon_{0}=0.8126$. Moreover, for $l / 2(\omega 2 / \pi \rho G)=$ 0.1871 the axes $a$ and $b$ become equal and the Jacobi ellipsoid turns into an ellipsoid of revolution, which at the same time is also a Maclaurin ellipsoid. For $l / 2\left(\omega^{2} / \pi \rho G\right)>0.1871$ triaxial ellipsoids of equilibrium of a rotating fluid do not exist.

The Maclaurin spheroid is considered to be the simplest model of rotating ellipsoidal figures in equilibrium since it assumes uniform density. However, heterogeneous mass distribution has been studied using concentric Maclaurin spheroids (CMS). The method has been mainly developed by Hubbard $(2012,2013)$ and is consisting of a numerical hydrostatic scheme which decomposes a rotating celestial body into $N$ spheroids of constant density (the initial Maclaurin spheroid would correspond to the case $N=1$ ). For a given density profile, the gravitational potential of each spheroid (which is constant on the spheroid) is calculated. Then is computed on a self-consistently way the radius of each spheroid as a function of latitude. Such a method has been developed for solar system planets, Mars, Juno, Neptune or Uranus. The method is somewhat limited; to fulfill the Juno's observations of the gravitational moments for instance, at least 1500 spheroids must be used [Debras and Chabrier, 2017]. It gives nevertheless very satisfactory results, the fit being up to the 3rd digit at least. The CMS method has never been applied to the Sun.

For a constant density oblate region, the $J_{n}$ can be analytically determined (through what is called the "exact" Maclaurin solution), that are [Klioner, 2003; Hubbard, 2012; Panhans \& Soffel 2014]:

$$
J_{n}=-\frac{3(-1)^{\left(1+\frac{n}{2}\right)}}{(n+1)(n+3)}\left(\frac{\ell^{2}}{1+\ell^{2}}\right)^{\left(\frac{n}{2}\right)}
$$

where $l^{2}$ is the ellipticity of the oblate body given $l=1-\mathrm{R}^{2}{ }_{\mathrm{eq}} / \mathrm{R}^{2}{ }_{\mathrm{pol}}, R_{e q}$ and $R_{p o l}$ being respectively the equatorial and polar radius ${ }^{3}$.

Note that $l$ is related to $f$ by $l^{2}=2 f-f^{2}$ or conversely $f=1-\left(l-l^{2}\right)^{1 / 2}$. As an example, taking $R_{e q}=695509.9835 \mathrm{~km}$ and $R_{p o l}=695504.0331 \mathrm{~km}$ in such a way that $R=\left(R_{e q}^{2} \times R_{p o l}\right)^{1 / 3}=$ $695508.0000 \mathrm{~km}$ (astrometric accuracy!), it comes $f=8.56 \times 10^{-6}$ and

\footnotetext{
${ }^{2}$ Figures of equilibrium of a rotating fluid exist which have the form of ellipsoids of revolution (Maclaurin ellipsoids) when $a=b>c$, and of tri-axial ellipsoids (Jacobi ellipsoids) when the axis $c$ is the smallest axis of the ellipsoid.

${ }^{3}$ There is a mistake in equation (27) of Panhans \& Soffel (2014) for $n=1$. It reproduces incorrectly equation (56) of Klioner (2003) which, in fact, contains $(1)^{n+1}$ instead of $(1)^{n}$ entering equation (27) of Panhans \& Soffel (2014).
} 


$$
J_{2}=-3.42 \times 10^{-6}, \quad J_{4}=2.51 \times 10^{-11}, \quad J_{6}=-2.39 \times 10^{-16}, \quad J_{8}=2.60 \times 10^{-21} \ldots
$$

Such values are crude order of magnitudes, as the density is taken as constant (solar mean value) and the differential rotation is not taken into account. However, they show that solar gravitational moments are rather faint and are decreasing as far as the order $\mathrm{n}$ is increasing. Note that Essen (2014), using a point core model found the following estimates:

$$
q=1.15 \times 10^{-5}, f=6.3 \times 10^{-6} \text { and } J_{2}=3.7 \times 10^{-7} .
$$

\subsection{Analysis through the gravity field}

As the outer equilibrium surface of a rotating star differs from sphericity, and even if in general the deviations are small, it results that the matter is not evenly distributed. The flattening of a star (or the Sun), for which we have a priori no knowledge of its stratification, limb boundary, planes of symmetry, transfer of angular momentum in a differentially rotating body, etc, implies to first order a bulge at the equator, and to higher orders deviations to sphericity. These excesses (or deficit) of mass are due to the existence of the gravitational moments $J n$ in the expression of the total potential of the body (sum of the gravitational potential and the potential of centrifugal forces). The even order $n=2$ is called the quadrupole moment $J_{2}$. Higher orders are called dodecapole $J_{4}$ (sometimes also called octupole), hexadodecapole $J_{6}$, etc...

\subsubsection{The gravitational potential}

can be expressed as:

$$
\Phi_{g}(P)=\Phi_{g}(x, y, z)=G \iiint\left(\frac{d m}{l}\right)=G \iiint\left(\frac{\rho}{l} d v\right)
$$

where $P(x, y, z)$ denotes the point at which $\Phi_{g}$ is calculated, $d_{m}$ is the mass element for which $\rho=d m / d v$ and $l=\left(x^{2}+y^{2}+z^{2}\right)^{1 / 2}$. The Laplace's equation $\nabla \Phi_{g}=0$, written in spherical coordinates $(r, \theta, \lambda)$ can be solved by a product of three functions, each of which depending on only one coordinate: $\Phi g=-f(r) g(\theta) h(\lambda)$, whose solutions are:

$$
\begin{gathered}
f(r)=r^{n} \text { or } r^{-(n+1)} \\
g(\theta)=P_{n m}(\cos \theta) \\
h(\lambda)=\cos (m \lambda) \text { or } h(\lambda)=\sin (m \lambda)
\end{gathered}
$$

where

$n=0,1,2,3, \ldots$, is called the degree and $m=0,1, \ldots n$, is called the order of the function under consideration. $P_{n m}$ is the Legendre polynomial of degree $n$, for a given $m$.

In 1875, A.M. Legendre published his book "Sur l'attraction des spheroides" in which he developed the gravitational potential in terms of a power series

$$
V(r)=-\frac{G M}{r}+\mathcal{O}\left(\frac{1}{r^{n}}\right)
$$

where $M$ is the total mass and $G$ the Gravitation constant $\left(G=6.67259(85) \times 10-11 \mathrm{~m}^{3} \mathrm{~kg}^{-1}\right.$ $\left.\mathrm{s}^{-2}\right) . O\left(1 / r^{n}\right)$ indicates that for $r \rightarrow \infty$, this term tends to zero as $1 / r^{n}$. Following this formalism, the gravitational potential $\Phi_{g}(r, \theta, \lambda)$ can be developed under the series

$$
\Phi_{g}(r, \theta, \lambda)=-\sum_{n-0}^{\infty} \sum_{m=0}^{\infty}\left[\frac{1}{r^{n+1}}\left(C_{n m} P_{n m}(\cos \theta) \cos (m \lambda)+S_{n m} P_{n m}(\cos \theta) \sin (m \lambda)\right)\right]
$$


which is also

$$
\Phi_{g}(r, \theta, \lambda)=-\sum_{n=0}^{\infty} \frac{1}{r^{n+1}} \sum_{m=0}^{\infty}\left[C_{n m} \cos (m \lambda)+S_{n m} \sin (m \lambda)\right] P_{n m}(\cos \theta)
$$

Since the first term $n=0$ is nothing else but $G M / r$ (see Eq. 3), it turns out that

$$
\Phi_{g}(r, \theta, \lambda)=-\frac{G M}{r}\left[1-\sum_{n-1}^{\infty} \sum_{m=0}^{\infty}\left(\frac{a}{r}\right)^{n}\left[C_{n m} \cos (m \lambda)+S_{n m} \sin (m \lambda)\right] P_{n m}(\cos \theta)\right]
$$

The coefficients $C_{n m}$ are called the tesseral coefficients; for $m=0, C_{n 0}$ are the zonal coefficients; $C_{20}$ (namely zonal harmonic of degree-2 order- 0 ) is the dynamical flattening of the body (but not the flatness, as sometimes written, mainly in planetary ephemerides papers), and is usually designed by $J_{2, \text { Sun }}$ or if no ambiguity, more simply by $J_{2}$. Note that the degree $n$ is linked with the wavelength $\varsigma$ of the mass anomalies inside the body: $n=2 \pi a / \varsigma$. The larger the $n$ are, the finer is the resolution.

For the general case, the rotating body takes the shape of a spheroid, which can be described by successive parameters, $f$ to first order (as above-mentioned), $h$ and $k$ for higher orders, and is either oblate (elongation along the equatorial axis, $n=1, m=1$-called oblateness-), or prolate (elongation along the polar axis $n=1, m=2$-called prolateness-. Figure 1 visualizes the different cases.

For the Sun (or stars), for which an axially symmetric distribution of the rotating matter can be assumed (not for the Earth), $S_{n m}=0, m=0$, so that Eq. 4 reduces to:

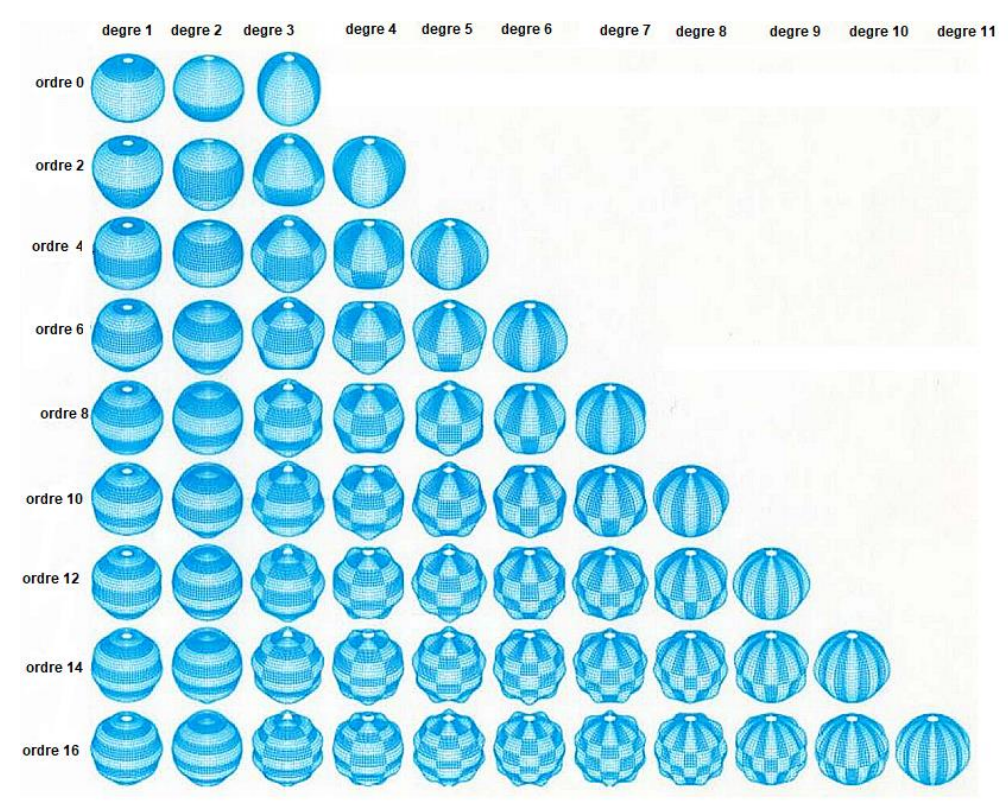

Figure 1: Laplace spherical harmonics showing the surface distortions of a rotating fluid body in the general case. The gravitational moment $J_{2}$ is given by its degree $n=2$ order $m=0$, which is the dynamical, flateness, $c_{20}$. (After R. Biancale, 2006 personal communication).

$$
\Phi_{g}(r, \theta)=-\frac{G M}{r}\left[1-\sum_{n=1}^{\infty}\left(\frac{a}{r}\right)^{2 n}\left[C_{2 n}\right] P_{2 n}(\cos \theta)\right]
$$


where $C_{2 n}$ are the degree $-n$ order -0 gravity field of the body under consideration. In the solar case, $n=2$ is commonly called Sun's gravitational degree 2 zonal harmonic, or more simply $J_{2}$ (which is not the oblateness but only an 'indicator').

\subsubsection{The centrifugal potential}

$\Phi_{c}$ is given by:

$$
\Phi_{c}=-\int_{0}^{r \sin (\theta)} \omega^{2} r \sin (\theta) d(r \sin \theta)
$$

for a body rotating at a velocity rate $\omega$, which is, integrated over the sphere $r=R_{s p}$

$$
\Phi_{c}=(1 / 2) \omega^{2} R_{s p}^{2} \sin ^{2} \theta=(1 / 3) \omega^{2} R_{s p}^{2}\left[1-P_{2}(\cos \theta)\right]
$$

\section{- The case study for a uniform rotation $\omega_{0}$}

From Eq. 1 and truncated to order 2, we get successively $1 / \mathrm{r}=\left(1 / R_{s p}\right) 1+2 / 3 f P_{2}+O\left(f^{2}\right)$ and the radius of the sphere $R_{s p}$, which is determined for $P_{2}=0: R_{s p}=R_{e q}(1-1 / 3 f)$. It results that the gravitational potential $\Phi_{g}$ can be expressed as a function of $R_{s p}$ as

$$
\Phi_{g}=-\frac{G M}{R_{s p}}\left[1+\frac{2}{3} f-J_{2} P_{2}\right], \text { accurate up to } \mathcal{O}
$$

The total potential is thus

$$
\Phi=-\frac{G M}{R_{s p}}\left[\left(1+\frac{1}{3} m\right)+\left(\frac{2}{3} f-J_{2}-\frac{1}{3} m\right) P_{2}\right],
$$

setting $\mathrm{q}=\omega^{2} R^{3}{ }_{s p} /(G M)$ as previously seen.

If the solar ellipsoid is a level surface, $\Phi$ must be constant on it; thus the coefficient of $P_{2}$ in Eq. 8 must vanish:

$$
\frac{2}{3} f-J_{2}-\frac{1}{3} q=0
$$

or

$$
J_{2}=\frac{2}{3} f-\frac{1}{3} q
$$

Using previous numerical values, inferring no differential rotation, and taking $\omega=2.85$ $\mu \mathrm{rad} / \mathrm{s}$ (i.e. the velocity rate at the equator), we get $q=2.05910^{-5}$, so that $J_{2}$ in this case and limited to order 2 is:

$$
J_{2}=-1.1610^{-6}
$$

\section{- Higher orders.}

Designing by $\mathrm{k}(\mathrm{Req})$ and $\mathrm{h}(\mathrm{Req})$ the second-order and third-order corrections to the equation of a spheroid whose semi-axes are Req and $\operatorname{Rpol}=\operatorname{Req}(1-f)$ is

$$
\begin{aligned}
& r(\theta)=R_{e q}\left[1-f \cos ^{2} \theta-\left(\frac{3}{8} f^{2}+k\right) \sin ^{2} 2 \theta+\right. \\
& \left.+\frac{1}{4}\left(\frac{1}{2} f^{3}+h\right)\left(1-5 \sin ^{2} \theta\right) \sin ^{2} 2 \theta+\cdots\right] .
\end{aligned}
$$


hence it comes:

$$
R_{e q}=s\left(1+\frac{1}{3} f+\frac{2}{9} f^{2}+\frac{8}{15} k+\frac{14}{81} f^{3}+\frac{26}{105} h+\frac{16}{63} f k\right)
$$

or conversely

$$
\mathrm{s}=R_{e q}\left(1-\frac{1}{3} f-\frac{2}{9} f^{2}-\frac{8}{15} k-\frac{5}{81} f^{3}-\frac{26}{105} h-\frac{32}{315} f k\right),
$$

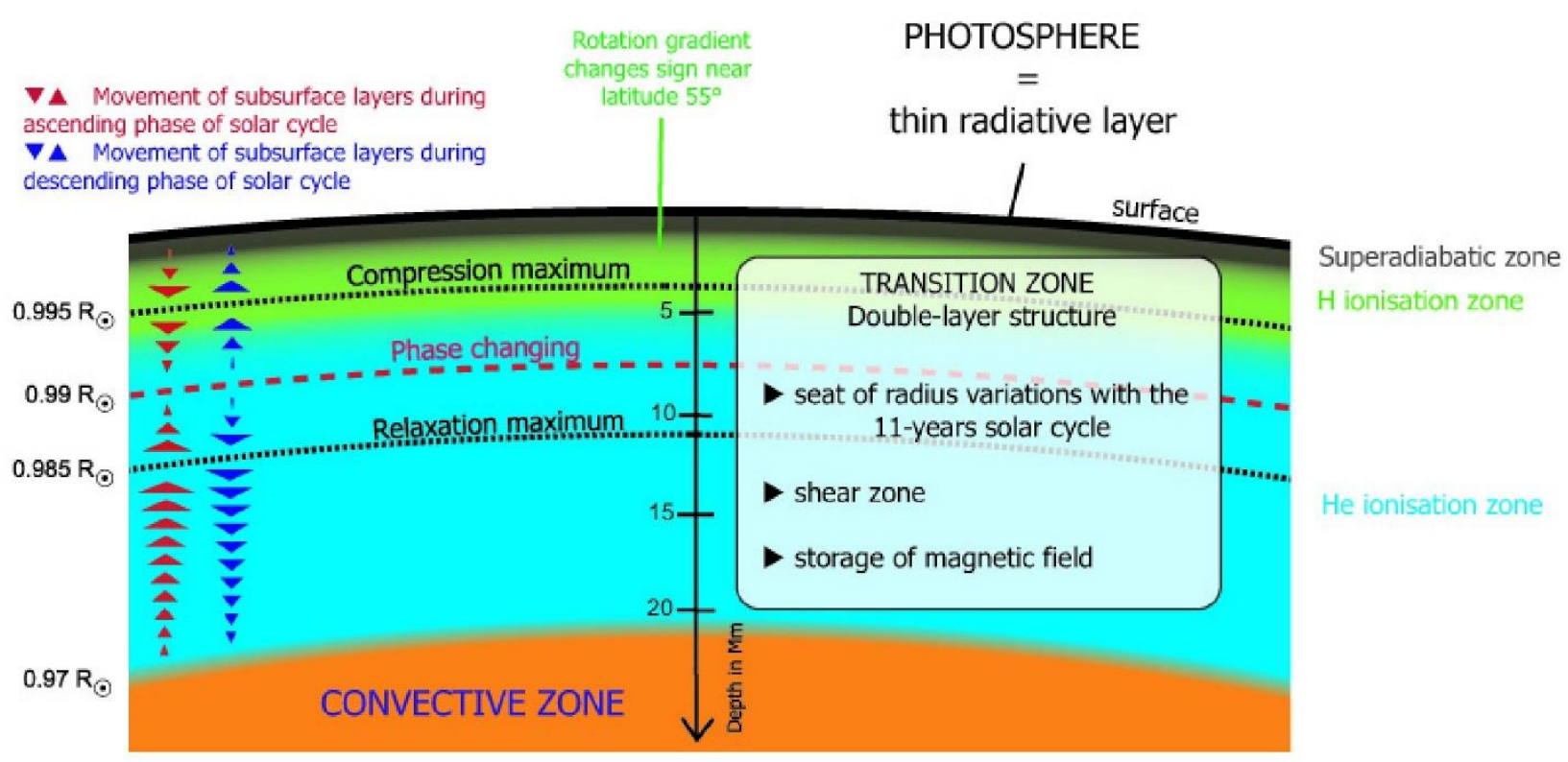

Figure 2: The differential rotation discovered at the surface of the Sun through spots is extended into the interior and is detected by helioseismology (left). Two main layers can be seen, the tachocline, seat of the differential rotation, and the leptocline, a near sub surface layer (NSSL) where signicant physical phenomena seem to be anchored (right): non-homologous radius changes in time and in depth, shearing, disturbance of the turbulent pressure, constraints upon the magnetic field, processes of ionization, etc.

After some algebra, the analytical form of the Jn can be found as:

$$
\begin{gathered}
J_{2}=+\left[\frac{2}{3} f-\frac{1}{3} q-\frac{1}{3} f^{2}+\frac{8}{21} k(1+q)+\frac{3}{7} f q+\frac{40}{147} f k-\frac{50}{294} f^{2} q-\frac{2}{21} h\right] \\
J_{4}=-\left[-\frac{4}{5} f^{2}-\frac{32}{35} k+\frac{4}{5} f^{3}-\frac{50}{49} f^{2} q+\frac{3616}{2695} f k-\frac{4}{7} f q+\frac{208}{385} q k-\frac{192}{385} h\right] \\
J_{6}=+\left[\frac{8}{7} f^{3}-\frac{20}{21} f^{2} q-\frac{160}{231} q k+\frac{128}{77} f k+\frac{80}{231} h\right] .
\end{gathered}
$$

\subsection{Case of the differential rotation}

The main difficulty in our problem comes from the differential rotation of the Sun, that is to say the variation of the rotation rate with latitude: the equatorial zones of the Sun rotate a little faster than the polar ones. This phenomenon has been detected immediately after the discovery of the sunspots when astronomers saw that their apparent motion took a little longer time to cross the solar disc at higher solar latitude than near the equator. The first major work 
on this subject was written in 1630 by Scheiner (Rosa Ursina sive sol) and since then, this physical phenomenon has never ceased to attract researchers mainly for two reasons: (i) its origin remains unsolved and (ii) it never stopped: in other words, there is no regulating process which would tend to standardize rotation over long periods of time.

The differential rotation is continuous in the sense that the rotation rate is slowly varying from the equator to the pole. This phenomenon goes further inside, down to the tachocline, a transition region interfacing the radiative interior and the differentially rotating outer convective zone. Helioseismology measurements indicate that the tachocline is located at a radius of at most 0.70 times the solar radius, with a thickness of 0.04 times the solar radius. The rotation rate through the interior is roughly equal to the rotation rate at mid-latitudes (i.e. approximately in-between the rate at the slow poles and the fast equator). Below the tachocline, the rotation is more uniform, but increasing with depth (see paragraph 4.1).

Solar rotation has been investigated by a variety of techniques. Observed data can be fitted through a law usually taken as

$$
\omega=\omega_{0}+\omega_{1} \sin ^{2}(\theta)+\omega_{2} \sin ^{4}(\theta)
$$

where $\omega_{0}$ is the angular velocity rate at the equator (and $\theta$ the heliographic latitude). The coefficient $\omega_{0}$ represents the equatorial rotation rate and the coefficients $\omega_{1}$ and $\omega_{2}$ measure the latitudinal gradient in the rotation rate, with $\omega_{1}$ representing mainly low latitudes whereas $\omega_{2}$ represents largely higher latitudes [Javaraiah 2009].

Equation 15 can be developed over the Legendre's polynomials basis, which leads to

$$
\omega=\left(\omega_{0}+\frac{1}{3 \omega_{1}}+\frac{1}{5 \omega_{2}}\right)+\left(\frac{2}{3 \omega_{1}}+\frac{4}{7 \omega_{2}}\right) P_{2}+\frac{8}{35 \omega_{2} P_{4}}
$$

Putting (16) into (7), the centrifugal potential is of the form

$$
\Phi_{c}=-\frac{1}{3} w_{0}^{2} \operatorname{Req}^{2}\left[A_{0}+A_{2} P_{2}+A_{4} P_{4}\right]
$$

The total potential $\Phi$, is the sum of the gravitational potential and the centrifugal one, $\Phi_{g}$ and $\Phi_{c}$, both depending on the radius $r$ and the colatitude $\theta$ :

$$
\Phi(r, \theta)=\Phi_{g}(r, \theta)+\Phi_{c}(r, \theta)
$$

which can be expressed as

$$
\Phi(r, \theta)=K\left[B_{0}+B_{2} P_{2}+B_{4} P_{4}+\ldots\right]+\mathcal{O}\left(f^{n}\right)
$$

On a level surface, $\Phi_{g}$ must be constant on it; thus the coefficient of $P_{n}$ must vanish, and the $J_{n}$ can be derived from $B_{n}=0$.

\section{Numerical expression}

Without removing generality, it is possible to compute $J_{n}$ by remembering that in Eq. 10 , it can be seen that the excess of its radius vector over that of the true ellipsoid is obtained at the 
colatitude for which the change occurs in the external contour shape, i.e. when the radial rotation gradient $\partial \omega / \partial \mathrm{r}$ changes in sign. Indeed $\partial \omega / \partial r$ is $<0$ at the equator up to $\theta \approx(40-55)^{\circ}$ and is positive thereafter ${ }^{4}$.

Adopting for instance the Javaraiah's (2002) law, $\omega=2.936-0.56 \sin ^{2}(\theta)$, it comes at $\theta=$ $46^{\circ}$

$$
J_{2}=-2.10 \times 10^{-7}, \quad J_{4}=1.45 \times 10^{-10} \text { and } J_{6}=-5.22 \times 10^{-16}
$$

which can be compared to the values obtained by Armstrong and Kuhn (1999):

$$
J_{2}=-2.22 \times 10^{-7}, J_{4}=3.84 \times 10^{-9}
$$

We can deduce the shape coefficients (limited to order 2) $s_{2}=-J_{2}-(1 / 3) * q$ and $s_{4}=-J_{4}+$ $(6 / 35) * q$, which are

$$
s_{2}=-5.71 \times 10^{-6}, s_{4}=3.04 \times 10^{-6}
$$

and again can be compared to the values deduced by Armstrong and Kuhn (1999):

$$
s_{2}=-5.84 \times 10^{-6}, s_{4}=0.593 \times 10^{-6}
$$

It can be checked that $f$, which is $\approx(3 / 2) s_{2}-(5 / 8) s_{4}$, is thus $6.66 \times 10^{-6}$ in the first case and $3.63 \times 10^{-6}$ in the second one (to be compared with the adopted value of $8.56 \times 10^{-6}$ ).

For the time being, we have no explanation for the discrepancy on the $n=4$ term. Armstrong and Kuhn (1999) have provided a more sophisticated analysis, taking into account the non uniform rotation over latitudinal cylinders. However, these authors have claimed that 'the quadrupole (and hexadecapole) limb shape are mildly inconsistent with current solar rotation models' and that the discrepancy will probably be removed by a better understanding of the solar core rotation. Progress on this problem will depend on improved measurements of the limb shape (through dedicated missions), but also by a better understanding of the twodimensional solar interior rotation rate. However, the $\mathrm{J} 2$ estimates, as well as their order of magnitudes, are in good agreement with these deduced from other methods. Thus Paterno (1996), Godier and Rozelot (2001), Pireaux and Rozelot (2003), Eren and Rozelot (2020, weighted estimate) found respectively (in $10^{-7}$ ), $-2.08 \pm, 0.14,-2.0 \pm 1.4,-2.00 \pm 0.40$ and $2.17 \pm 0.06$. Pijpers (1998) found $+2.18 \pm 0.06$; if the order of magnitude is respected, the sign is not and this is not only a matter of convention: its value is questionable. From observations, the discrepancies found may be due to the temporal dependence of the gravitational moments as will see latter on. Thus we emphasize the need to accurately measure the limb shape and its variation with time.

\section{- Second order analysis of the differential rotation.}

The usual centrifugal potential $\Phi_{c}$ must be rewritten to take into account the differential rotation on a physical point of view; in this case, the problem is no longer conservative, and the star is baroclinic. However, we can postulate that the centrifugal force must derive from a potential, in such a way that it must be possible to find a function $U$ which satisfies (Lefebvre 2003):

$$
\vec{F}_{\text {centrifugal }}=-\vec{\nabla} U
$$

At a depth $r_{p}$, one can use an equation of the form

\footnotetext{
${ }^{4}$ The order of magnitude of this outward gradient is $5.7 \times 10^{16} \mathrm{~m}^{-1} \mathrm{~s}^{-1}$ at the equator.
} 
and $\omega_{p o l}=2.399 \mu \mathrm{rad} / \mathrm{s}$.

$$
\Omega=\Omega_{p o l}\left[1+\sum_{i=1}^{\infty} a_{2 i} r_{p}^{2 i} \cos ^{2 i}(\theta)\right]^{\frac{1}{2}}
$$

which derives from a potential. Using the solar Greenwich database that records the sunspots position as a function of time, Javaraiah and Rozelot (2002) have computed $a_{i}$ for $i=1$ and $i=$ 2, which are:

The reader will be able to verify the perfect adjustment of the two curves given by Eq. 15 and 18, using the numerical values given here. Fig. 3 shows the rotation rate with depth (from $r_{p}=1$ down to $0.75 R$ ). The inversion of the radial gradient rotation rate can be seen at $\theta=37^{\circ}$ of latitude, within the leptocline. This mechanism signs the main difference with a stellar structural approach and put in evidence the key role of the solar gravitational moments $J_{2}$ and $J_{4}$.

Best known estimates of $\omega 0, \omega 1$ and $\omega 2$, according to several authors, are given in Table 2 published in [Rozelot 2003b]. The following two examples differ from the way sunspots and faculae rotation were analyzed (coefficients in $\mu \mathrm{rad} / \mathrm{s}$ ):

$\omega 0=2.913, \omega 1=-0.283$ and $\omega 2=-0.269$ (Kuiper 1972),

$\omega 0=2.82, \omega 1=-0.33$ and $\omega 2=-0.53$ (Howard 1980).

Numerical values are of importance, as a small change could have a relatively large effect on the implied multipolar moments.

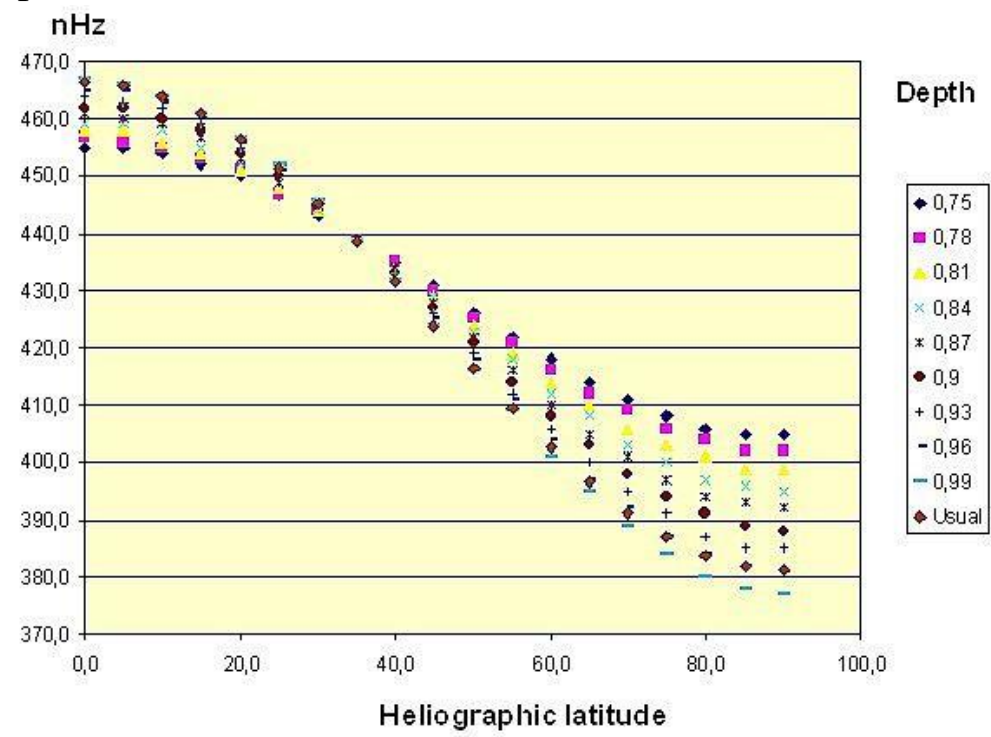

Figure 3: Differential rotation (velocity rate in $n \mathrm{~Hz}$ versus the heliographic latitude), for the solar case and according to a law deriving from a potential. The different depth are listed in the right box. One can perfectly see the inversion of the radial gradient of rotation at $\theta=37^{\circ}$ of latitude (Influence of the leptocline at 0.99 R. See Lefebvre et al., 2007).

The rotational potential $\Phi_{c}$ can then be expressed in terms of Legendre's polynomials

$$
\Phi_{c}=-\chi \frac{G M}{R_{s p}}\left[A_{0}+A_{2} P_{2}+A_{4} P_{4}\right]
$$


with $\chi$ defined as $\chi=\omega^{2}{ }_{e q} R^{3}{ }_{e q} / G M$. (Note that $q$ and $\chi$ are related in terms of each other and are expressed in terms of $s_{0}, s_{2}, s_{4} \ldots$, as:

$q / \chi=(R e q / s)^{3}=1-3 / 2 s_{2}+\left(3 / 4 s^{2}{ }_{2}+3 s_{0}+9 / 8 s_{4}\right)+\ldots$, . It turns out that

$A_{2}=\left[\left(-\frac{46832}{135135} a_{4}-\frac{3064}{10395} a_{2}-\frac{82}{315}\right) f^{2}+\left(-\frac{160}{693} a_{4}-\frac{16}{63} a_{2}-\frac{20}{63}\right) f+\left(-\frac{8}{63} a_{4}-\frac{4}{21} a_{2}-\frac{1}{3}\right)\right]$

and

$$
A_{4}=\left[\left(\frac{9552}{25025} a_{4}+\frac{77456}{225225} a_{2}+\frac{76}{231}\right) f^{2}+\left(\frac{96}{455} a_{4}+\frac{256}{1155} a_{2}+\frac{8}{35}\right) f+\left(\frac{24}{385} a_{4}+\frac{2}{35} a_{2}\right)\right]
$$

Writing that on the surfaces of constant potential $\Phi$ must not depend on the heliographic latitude, it comes after computations, and accurate up to $O\left(f^{3}\right)$

$J_{2}=\frac{2}{3} f-\frac{1}{3} f^{2}+\chi A_{2}-\frac{26}{21} \chi A_{2} f-\frac{20}{21} \chi A_{4} f+\frac{521}{2205} \chi A_{2} f^{2}+\frac{3350}{4851} \chi A_{4} f^{2}+(\ldots)$

and

$J_{4}=-\frac{4}{5} f^{2}+\chi A_{4}-\frac{36}{35} \chi A_{2} f-\frac{502}{231} \chi A_{4} f+\frac{8309318}{3468465} \chi A_{4} f^{2}+\frac{4866}{2695} \chi A_{2} f^{2}+(\ldots)$

where $A_{2}$ and $A_{4}$ are determined by the $a_{\mathrm{i}}$.

With the numerical values already given, one obtains $A_{2}=-0.42(4638)$ and $A_{4}=+$ $0.028(751)$ at $r_{p}=1 R$

It is obtained in such a way $J_{4}=(4.36 \pm 0.2) 10^{-7}$.

Another approach is to develop the solar rotation described by Eq.15, by means of a set of disc-orthogonal functions:

$\mathrm{T}^{0}{ }_{1}(\sin \theta)=1$,

$\mathrm{T}^{1}{ }_{2}(\sin \theta)=5 \sin ^{2} \theta-1$, and

$\mathrm{T}^{1}{ }_{4}(\sin \theta)=21 \sin ^{4} \theta-14 \sin ^{2} \theta+1$,

which leads to the following expansion:

$$
\omega(\theta)=\bar{A}+\bar{B}\left(5 \sin ^{2} \theta-1\right)+\bar{C}\left(21 \sin ^{4} \theta-14 \sin ^{2} \theta+1\right) .
$$

The coefficients $A, B$, and $C$ are free of crosstalk, $A$ represents the 'rigid body' (or 'mean') component in the rotation, $B$ and $C$ are the components of the differential rotation. If the polynomial expansion is terminated at $C$, the coefficients $A, B$, and $C$, are related to the standard $A, B$, and $C$ coefficients as follows:

$\bar{A}=A+\left(\frac{1}{5}\right) B+\left(\frac{3}{35}\right) C$,

$\bar{B}=(1 / 5) B+2 / 15) C$,

$\bar{C}=(1 / 21) C$.

Using Legendre polynomials $P_{0}, P_{2}$ and $P_{4}$ as a set of orthogonal functions, the differential rotation can be described by:

$$
\omega(\theta)=D P_{0}+E P_{2}(\cos \theta)+F P_{4}(\cos \theta) .
$$


If the expansion is truncated at the third term, the coefficients $D, E$, and $F$ are related to the coefficients $A, B, C$ in Eq. 25 as follows:

$$
\begin{aligned}
& D=A+(1 / 3) B+(1 / 5) C, \\
& E=(2 / 3) B+(4 / 7) C, \\
& F=(8 / 35) C .
\end{aligned}
$$

- Discussion. The accurate determination of the $J_{4}$ term is not easy, as it can be seen from several papers already published on this question. Taking into account the role of the solar magnetic field, it has been found $J_{4}=6.2911^{-7}$ (Ajabzirizadeh et al., 2008; error bars can be set up at $2.010^{-7}$ ).

These values are an order of magnitude less that those obtained through the theory of stellar structure (see Pireaux and Rozelot, 2005). In the helioseismology analysis, the kernels for the high-order multipole moments are more concentrated near the surface, and may be responsible for the difference. However, our estimate are consistent with observations of Lydon \& Sofia (1996), leading to $J_{4}=9.83 \times 10^{-7}$ (and $J_{2}=1.84 \times 10^{-7}$ ).

We think that the contribution of $J_{4}$ - if its value is confirmed to be of the same order of magnitude than $J_{2}-$ is a key to explain solar observations. Let us report here the results obtained from space missions:

1. Emilio et al. (2007) reported a solar shape distortion using the Michelson Doppler Imager (MDI) aboard the Solar and Heliospheric Observatory (SOHO) satellite, after correcting measurements for bright contamination. It was found that the shape distortion is nearly a pure oblateness term in 2001, while 1997 has a significant hexadecapolar $\left(J_{4}\right)$ shape contribution (Fig. 4).
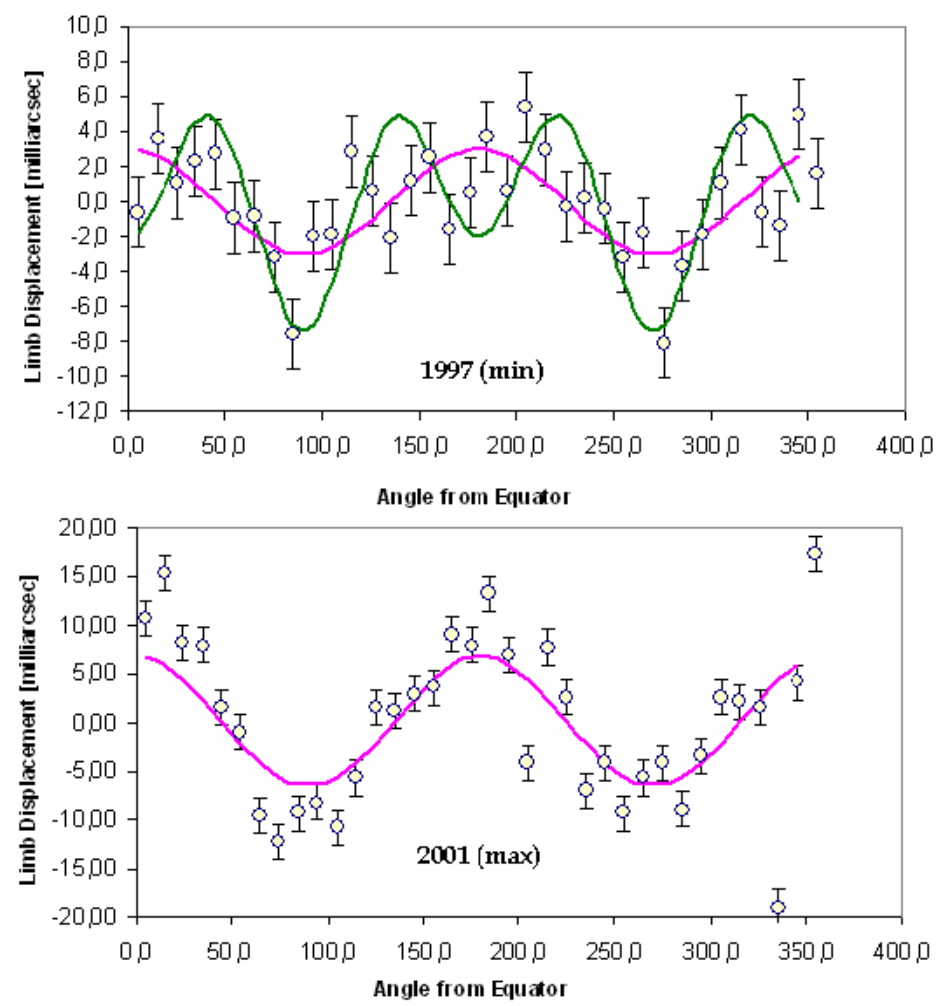

Figure 4: The solar shape variations in time deduced from SOHO. The oblateness contribution (linked to the quadrupolar term $J_{2}$ term) is the only one in 2001, albeit a significant hexadecapolar $\left(J_{4}\right)$ contribution is predominant in 2007. (After Emilio et al., 2007).

2. Antia et al. (2008) analyze on a timescale of the solar cycle, the variation of the angular momentum of the Sun and the associated variations in the gravitational multipole moments, by inverting helioseismic rotational splitting data (obtained by the Global Oscillation Network 
Group and by the Michelson Doppler Imager on the Solar and Heliospheric Observatory. They found a temporal variation in angular momentum at high latitudes $\left(>45^{\circ}\right)$ through the convection zone positively correlated with the level of solar activity, whereas at low latitudes it is anticorrelated, except in the top $10 \%$ by radius where both are correlated positively. The variation (in relative value, not in absolute one) is less than the one we found from the sinusoid fitted to the $s_{4}$ coefficients derived from observations. The difference could partly originate from errors in the distortion observed, but it is likely to be due to the direct effects of the centrifugal force and the magnetic field.

3. Observations from RHESSI [Fivian et al. 2008] shows in the data obtained since 2004, an excess of oblateness of around 2 mas that the authors attribute to the EUV limb brightness. This excess could be merely the increase of oblateness due to the differential rotation. In other words, the excess could be explained by the combined contribution of the oblateness (quadrupolar term) and the hexadecapolar term.

It results from this discussion that the solar shape is nearly purely oblate near solar maximum, but has a signicant hexadecapole shape near minimum.

\section{Exploring the Temporal Variation of the Solar Gravitational Moments}

Various authors have independently developed high precision of Lunar and Planetary Ephemerides, which has served as a basis to set up celestial and nautical almanacs. They are considered as a worldwide resource for fundamental astronomical data, often being the rst publications to incorporate new International Astronomical Union resolutions. Planetary Ephemerides are developed on the basis of numerical integration of the motion of the nine planets and the Moon fitted to the most accurate available observations. They progressively integrated the motion of perturbing main belt asteroids (up to 300), the Earth's rotation and Moon libration. These datasets comprise of a variety of planetary observations, such as, spacecraft radio science and tracking data (VLBI), direct radar measurements, lunar laser ranging (LLR), astrometric photographs, CCD and occultations to name a few. $J_{2}$ is obtained as a sub-product of the fitting process assuming a complete consistency of the dynamical modeling since Earth rotation, Moon libration and asteroid orbits are integrated with the main equations of the planetary motions (see for instance a discussion by Hilton and Hohenkerk, 2010). The $J_{2}$ estimate has thus been regularly published as shown in Table 1.

A careful analysis of this set of data leads to a possible temporal dependance of $J_{2}$ as shown in Fig. 5, redrawn from Fig.1 taken in Eren \& Rozelot (2020).

A rather complete approach was first made by Komm et al. (2003), who derived from helioseismic data a temporal variation of the coefficient of $P_{4}$ (in the Legendre expansion of the rotation law), indicative of torsional oscillation patterns extending deep into the convection zone, while the angular momentum shows a 1.3-year period that hints at a long-term trend likely related to the solar activity cycle. Antia et al. (2004) found also through an analysis of the helioseismic rotational splitting data a significant temporal variation in the angular momentum and in the gravitational multipole moments. For these authors, the quadrupole moment $J_{2}$ exhibits no noticeable temporal variation, while $J_{4}$ up to $J_{12}$ do show a stronger variability.

To interpret the discrepancy with the Armstrong and Kuhn (1999) results, Antia et al. (2008) argue that "most of the distortion from sphericity is the direct response to the centrifugal force on the rotating surface layers, and not from the asphericity of the gravitational field". This argument is in contradiction with Dicke's statement: "a solar ellipticity requires a rotating distorted gravitational potential function, the most likely being a fast rotating solar core. There are only two feasible sources of internal stress to distort the solar core, a magnetic field and a Reynolds stress (fluid motion). The latter does not readily yield a rotating distortion, but a magnetic field demands it". Investigations of the properties of the solar interior provide 
additional indirect support, both experimental and theoretical, for the hypothesis that the Sun may contained a decoupled rapidly rotating core. Such a premise seems confirmed by Fossat $e t$ al. (2017) who analyzed the very low frequency $g$ modes in the asymptotic regime by statistical means of the GOLF measurements on-board the SOHO satellite [Gabriel et al. 1995]. They showed a rapid rotation frequency of $1644 \pm 23 \mathrm{nHz}$ of the solar core, which is a factor of 3.8 \pm 0.1 faster than the rotation of the radiative envelope. If such a fast solar rotator is confirmed, for a rigid rotation, this would imply a high solar core quadrupole moment (of about $3.6 \times 10^{-5}$ ), in any case greater than the one which has been observed up to now (at the surface, but integrated from the core). The question which arise, and presently into consideration, is how to take into account such an inside quadrupole moment with an integrated one of lower estimate. Two possibilities occur: one is through the Brans-Dicke theory, for which it would be conceivable to determine the $v$ coupling factor; the other one would be through an internal magnetic field compatible with the axial symmetry required by a field locked in the rotating core. Such a rapid rotation has not been confirmed and remains difficult to explain by models describing a pure angular momentum evolution without adding new dynamical processes such as internal magnetic breaking, which could have appeared when the Sun was young [TurckChieze et al. 2010]. Such an interpretation is requiring further theoretical work which is also in progress.

Analysis of the perihelion precession of planetary orbits computed in the solar equatorial coordinate system, instead of the ecliptic coordinate system usually used, shows that a periodic variation of the $\mathrm{J} 2$ term rather than of a simple constant, must be considered, and can be described as followed [Xu et al., 2017].

\begin{tabular}{|c|c|c|}
\hline $\begin{array}{l}\text { Name of the ephemeris } \\
\text { \& Institute }\end{array}$ & $\begin{array}{l}\text { Quadrupole moment } \\
\text { (in } 10^{-7} \text { ) }\end{array}$ & Author \& Refer \\
\hline DE405 JPL (USA) & $1.9 \pm 0.3$ & Standish 1998 \\
\hline EPM2004 IAA (Russia) & $1.9 \pm 0.3$ & $\begin{array}{l}\text { Pitjeva 2005; } \\
\text { Pitjeva } 2013\end{array}$ \\
\hline INPOP06 IMCCE (F) & $1.95 \pm 0.55$ & Fienga 2011 \\
\hline EPM2008 IAA (Russia) & $1.92 \pm 0.30$ & Pitjeva $2014 a$ \\
\hline INPOP08 IMCCE (F) & $1.82 \pm 0.47$ & Fienga 2011 \\
\hline DE423 JPL (USA) & 1.80 & Folkner 2015 \\
\hline INPOP10e IMCCE (F) & $1.8 \pm 0.25$ & Verma 2014 \\
\hline EPM2011 IAA (Russia) & $2.0 \pm 0.2$ & Pitjeva 2014a \\
\hline DE430 JPL (USA) & $2.1 \pm 0.7$ & Folkner 2015 \\
\hline EPM2013 IAA (Russia) & $2.22 \pm 0.23$ & Pitjeva $2014 a$ \\
\hline INPOP13a IMCCE (F) & $2.40 \pm 0.20$ & Verma 2014 \\
\hline INPOP13c IMCCE (F) & $2.3 \pm 0.25$ & Fienga 2015 \\
\hline MESSENGER JPL (USA) & $2.26 \pm 0.09$ & Park 2017 \\
\hline
\end{tabular}

Table 1: Estimates of the solar quadrupole moment $J_{2}$, with their uncertainties, deduced from the Ephemerides. IAA: Institute of Applied Astronomy in Saint Petersburg (Russia). IMCCE: Institut de Mecanique Celeste et de Calcul des Ephemerides in Paris (F). INPOP13c is an upgraded version of INPOP13a, fitted to LLR (Laser Lunar Ranging) observations, including new observations of Mars and Venus deduced from MEX, Mars Odyssey and VEX tracking data. 


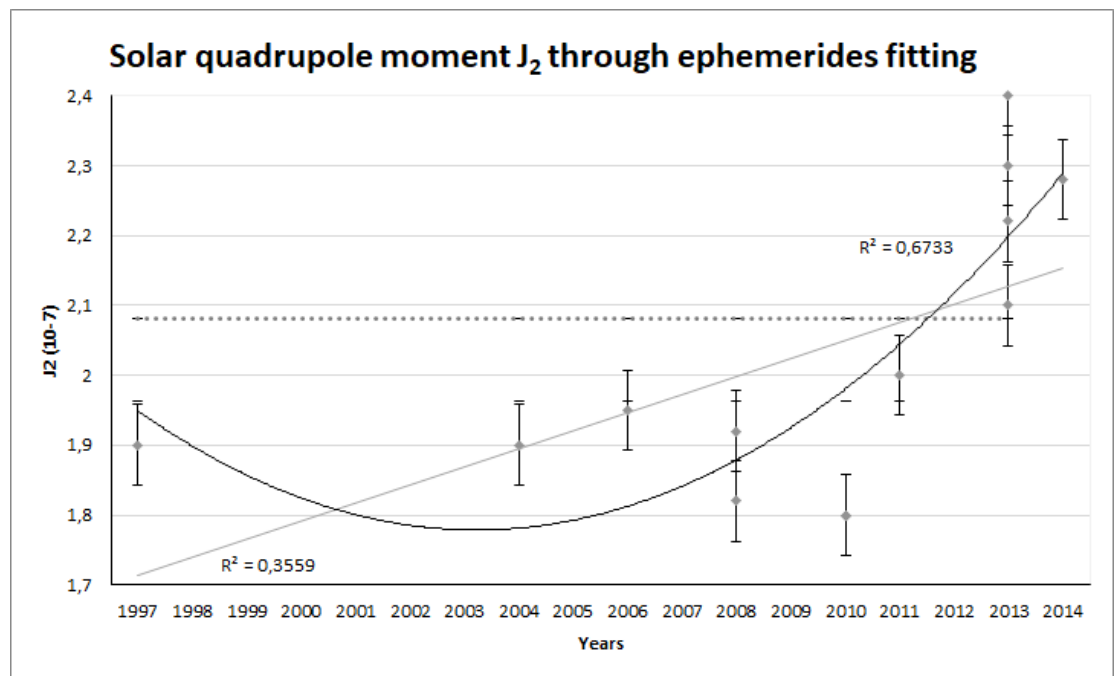

Figure 5: Solar quadrople moment $J_{2}$ estimates obtained as a sub-product of analytical solutions for the motions of planets deduced from high precision Lunar and Planetary Ephemerides. The best fit is a quadratic curve, but to be confirmed, would require a longer time scale. The main interest lies in the visible trend which is far from the (horizontal) doted line which shows the weighted mean estimate over the ranging time.

According to its time dependence, the $J_{2}$ term is redefined as $J_{2}=J_{20}+J_{21}$, where $J_{20}$ remains as the constant part of $J_{2}$ while $J_{21}$ denotes the temporal variation of $J_{2}$ and can be expressed as $J_{21}=A \cos \left(\omega J_{21} t\right)+B \sin \left(\omega J_{21} t\right)=\left(A^{2}+B^{2} \sin \left(\omega J_{21} t+\phi\right)^{1 / 2}\right.$. The disturbing part of the heliopotential of Eq. 5 can be written as

$$
\Phi=-\frac{G R_{e q}^{2}}{2 r^{3}} J_{2}\left(3 \sin ^{2} \theta-1\right)
$$

so that the secular effects of the solutions obtained by the integration for the Lagrangian equations of planetary motion can be developed according to $J_{21}$. Results are given in Xu et al. (2017) and the perihelion precession of Mercurys orbit caused by the periodic variation of $J_{2}$ is given in Fig. 6. It can be seen that the maximum perihelion precession of Mercurys orbit caused by a periodic variation of $J_{2}$ (of one solar cycle of 11.4 year, as suggested by Dicke, Kuhn \& Libbrecht (1986) or Rozelot, Damiani \& Pireaux (2009b)) is about $1700 J_{20}$, which is nearly 0.8 per cent of the secular perihelion precession.

\section{Relevance of accurate solar gravitational moments 4.1.Solar Astrophysical Quantities}

A number of questions are still pending about solar global properties. Among them: what is the influence of solar core dynamics on the values of the global solar spin $[J]$, on the multipolar moments $\left[J_{n}\right]$ and on the solar shape coefficients $\left[s_{n}\right]$ (which reflect the internal nonhomogeneous mass distribution and non-uniform angular velocity)? How to reconcile different estimates of $J_{n}$ ? How may the temporal dependence of $J_{n}, c_{n}$, and $J$ with the solar cycle affect the planets' motion?

All of these quantities are at the crossroad of solar physics, astrometry, and celestial mechanics. From the point of view of solar physics, their values reflect the physics of solar models: non-rigid rotation, solar latitudinal rotation, solarcore properties, solar-cycle variations, and stellar evolution. The knowledge of their temporal variations is essential to set constraints on solar-cycle modeling or solar-evolution theories. Another issue addresses the response of the rotating body: why it does include higher multipole moments than the first one which is represented by the rotational potential $\left[(1 / 2) \omega^{2} \sin ^{2}(\theta)\right]$ ? Hubbard (1975) postulated that the 
"higher moments would be zero if there were no contribution from the most outer shell, or, in other words, that the quadrupole response to a quadrupole perturbation does not produce a purely quadrupole structure in the shell, which, in turn excites the higher multipole moments".

In anycase, the anomalous precession of the orbit of Mercury and other planets, primarily due to GR effects, could be reconsidered through corrections due either to the presence of the Telisto/Planet Nine or that of a rapid solar core. More stringent tests rely on measuring the orbital precessions with much greater precision.

\subsection{Relativistic Astrometry}

Space-time is shaped by the presence of solar system bodies. The space-time curvature induced by the Sun leads to light deflection or corresponding timedelays in the propagation of signals. Precise astrometry in the solar neighborhood will thus require precise knowledge of the solar quadrupole moment and spin. Indeed, in addition to the solar mass monopole contribution $(\approx 1.75$ arcsec light deflection at grazing incidence $)$, there is a quadrupolar one $(\approx 0.4-0.3$ $\mu \operatorname{arcsec}$ at grazing incidence) and rotational solar contribution $(\approx \pm 0.7 \mu \operatorname{arcsec}$ at grazing incidence) [Pireaux 2002]. Unfortunately, the contribution of the $J_{2}$-term to light deflection drops dramatically as the angle of incidence (i.e. the closest approach to the Sun) increases (non-grazing incidence). Hence arises the need for determining an accurate $J_{2}$.

\subsection{Relativistic Celestial Mechanics}

The solar quadrupole moment also plays a role in celestial mechanics. The avance of the relativistic precession of the perihelion of planets is a known phenomenon [Pireaux and Rozelot, 2003], $\approx 43$ arcsec per century in the case of Mercury (exactly 42 ' '9794). As planetary ephemerides are today fitted to observational data sets (tracking data of spacecrafts, VLBI angular positions, or flyby normal points), a precise knowledge of $J_{2}$ and its variation are necessary to improve the fitting process. The statistics of the obtained postfit residuals may help to better constrain ephemerides. As an example, the knowledge of Mercury's orbit has made significant progress using this method, reaching a few meters to be compared with $800 \mathrm{~m}$ obtained through direct radar ranging [Cavanaugh et al., 2007].

Furthermore, the gravitational field associated with the mass quadrupole of the Sun creates a Newtonian perturbation on the orbit of the planets that has an effect on planetary spins and on the ecliptic plane. Up to now, the terms that contain the contribution to $J_{2}$ implicitly assume that the Sun's equatorial plane and the orbital plane of the planet in question coincide, which is an approximation. Here also, progress must be undertaken even if some work has been done in this direction [Iorio, 2011; Xu, 2011]. Finally, through solar system spin-orbit coupling, $J_{2}$ and $J$ will indirectly influence the orbital parameters of solar system bodies. For example, the Moon-Earth spin-orbit coupling propagates the influence of the solar quadrupole moment to the Moon. This has allowed, as soon as 1997, to set a dynamic upper limit to the solar quadrupole moment of $J_{2}$ to be $\leq 3 \times 10^{-6}$ [Rosch and Rozelot, 1996, Rozelot and Rosch, 1997; Rozelot and Bois, 1997; Bois and Girard, 1999], through observed lunar librations, but a more improved approach remains to be made.

\subsection{Possible Alternative Theories of Gravitation?}

Presently, there is still a strong correlation between post-Newtonian parameter $[\beta]$ and $J_{2}$ in planetary ephemerides. Hence, one cannot fit simultaneously for those two parameters, but a better knowledge of $J_{2}$ would thus help long-term Solar system modeling.

Indeed, together with $[\gamma]$, which encodes the amount of curvature of spacetime per unit restmass, the post-Newtonian parameter $[\beta]$ contributes to the relativistic precession of planets. The latter parameter encodes the amount of non-linearity in the superposition law of gravitation (with $\beta \equiv 1$ in General Relativity (GR)). Using a reasonable value for $J_{2}$ and present best 
constraints on post-Newtonian parameters $\gamma$ and $\beta$, GR is still in the contention, but there is room for alternative theories too [Pireaux and Rozelot, 2003]. Today, the best values available for $\beta$ and $\gamma$ are

$(\gamma-1) \times 10^{5}=2.1 \pm 2.3$ [Cassini mission, Bertotti et al., 2003]

$(\beta-1) \times 10^{4}=1.2 \pm 1.1$ [Lunar Laser Ranging, LLR: Williams et al., 2009, Equation 27]

and $\eta \times 10^{4}=(4 \beta-\gamma-3)=4.4 \pm 4.5$, through LLR also [Williams et al., 2009, Equation 26].

By accurately measuring the perihelion advances of several planets, it will be possible in the near future to decorrelate all of these quantities. Such a test is the purpose of several space missions, such as Beppi-Columbo, Gaia and Lator. This last mission has for its primary objective the measurement of the key post-Newtonian Eddington parameter $\gamma$ with an accuracy of one part in $10^{9}$ (a factor 30000 beyond the present best result obtained by means of Cassini [Bertotti et al., 2003]. Direct measurement of the solar quadrupole moment [ $\left.J_{2}\right]$ to an accuracy of one part in 200 of its size of around $10^{-7}$ is expected [Turyshev et al., 2009].

Currently, a first analysis of the Messenger flybys (Iorio 2011) shows that the LenseThirring precession in the case of Mercury may have been canceled to a certain extent by the competing precession caused by a small mismodeling in the quadrupole mass moment $\left[J_{2}\right]$ of the Sun, which could be estimated at $1.8 \times 10^{-7}$, thus more compatible with the RHESSI results $\left(J_{2}=(1.46 \pm 1.0) \times 10^{-7}\right.$ : Fivian et al., 2008).

\subsection{Accurate determination of the perihelion advance of a planet}

The first time that the solar quadrupole moment was associated with the gravitational motion of Mercury was in 1895, when Newcomb (1895 attempted to account for the anomalous perihelion advance of this planet with a modified gravitational field manifested by an oblateness $f$ of the Sun (the difference between the equatorial and polar radius $\Delta r$, reported to the equatorial one). Indeed, a few years before in 1859, Le Verrier had observed a deviation of Mercury's orbit from Newtonian's predictions, that could not be due to the presence of known planets. But, the difference between the equatorial and polar diameters of the Sun of $\Delta r=500$ mas (milli-arc-sec), as advocated by Newcomb, was soon ruled out by solar observations. And Einstein's new theory of gravitation, General Relativity, could account for almost all the observed perihelion advance. So, Mercury's perihelion advance readily became one of the cornerstones for testing General Relativity; even though, now, a contribution to the perihelion shift from the solar figure (though very less important than first suggested by Newcomb) cannot be discarded.

Keplerian laws of planet motions are solutions of the $n$-body gravitational problem. However, solar gravitational moments distort the gravitation force acting on the planets and disturb their Keplerian motions. Analytic solution of a planet orbit disturbed by the solar gravitational moments (solar oblateness, but also star oblateness in the case of exoplanets) can be derived. Except short -and long- periodic disturbances, secular disturbances lead to a perihelion precession and a nodal regression as well as a mean motion advancing. The magnitudes of the short-periodic perihelion precession could disturb the observation of the secular effect if the survey is made shorter than one Julian year. Transformation of the formulas from the solar equatorial plane to the ecliptic one has been discussed by $\mathrm{Xu}$ et al. (2011) who deduced the Mercury's, Venus' and Mars' secular perihelion precession as a function of $J_{2}$. Numerical estimates in the following are in $\operatorname{arcsec}$ per Julian century (as/Jc) taking $J_{2}=2 \times 10^{-7}$ [Pireaux \& Rozelot 2003, Pitjeva 2005]:

- perihelion precession of Mercury's orbit: $\Delta \omega_{1}=2.95694 \times 10^{5} J_{2}$, which is $0.0591(\mathrm{as} / \mathrm{Jc})$, 
- perihelion precession of Venus's orbit: $\Delta \omega_{1}=6.28801 \times 10^{4} J_{2}$, which is $0.01258(\mathrm{as} / \mathrm{Jc})$,

- perihelion precession of Mars's orbit: $\Delta \omega_{1}=2.95694 \times 10^{3} J_{2}$, which is 0.0013 (as/Jc).

Such numerical values are in good agreements with published results, namely those of Iorio (2004), noticing that the observations for the perihelion precession must be done long-yearly to be not disturbed by the short-periodic effects. Inversely, the solar oblateness could be determined through observation of perihelion precession of a planet.

\section{Conclusion}

We emphasized here the need to accurately capture real solar latitudinal intensity limb variations over a long period of time, a still challenging task. We highlighted that limb's tiny departure from perfect circularity, i.e. the asphericity, is sensitive to the Sun's otherwise invisible interior conditions ${ }^{5}$ allowing us to learn empirically about flows and motions there that would otherwise only be guessed from theoretical considerations. We then recalled that the knowledge of precise solar gravitational moments $\left[J_{n}\right]$ and their temporal dependence, can be used for the precise determination of solar parameters such as the angular momentum and its variation on the long term, that may help to locate the seat of the dynamo [Komm et al., 2003]. The observed oblateness temporal variations, which remain very faint (a few mas in amplitude), lag the solar activity cycles by about 3 years. A major change, which could be due to a possible exchange in magnitude between the two first solar moments ( $n=2$ and 4), occurred between solar cycles 23 and 24: the oblateness was greater in cycle 24 despite the lower solar activity level [Rozelot and Kosovichev, 2020].

Furthermore, $J_{2}$ at first, and surely $J_{4}$, are at the crossroads of relativistic astrometry and relativistic celestial mechanics. Indeed, (i) one of the major effects of a dynamical flattening of the Sun due to its oblique rotation with respect to the ecliptic plane is a secular variation in the orbital elements of the planet, which is not negligible, and must not be neglected in the computation of the ephemerides of celestial bodies. And (ii) they must be used, via their decorrelation with PPN parameters, as tests of alternative theories of gravitation.

Detailed analysis of the photospheric intensity distribution, including the brightness at the extreme limb, is important to determine the solar shape and its underlying parameters, shape coefficients, and oblateness, for which the most likely value of $\Delta r$ is 7.77 mas at null activity (best observed values give 8.21 mas \pm 1 mas, enclosing the theoretical estimate). The accurate knowledge of all above mentioned quantities is certainly of broad importance in solar and stellar physics, mainly concerning solar-cycle modeling and time evolution. The possible brightness variations include not only the classical limb-darkening function but also several other capabilities that enable us to deal with both the surface and the interior structure as well. The analysis must completely eliminate possible contributions to the solar photospheric intensity from an abundance of unresolved magnetic elements of very low magnetic flux density. First results obtained so far in this way from space missions (such as SDO - Solar Disk ObservatoryScherrer et al., 2012) seem to be reached. Furthermore, associated with other space missions dedicated to test the curvature of the solar system's gravity field with an accuracy better than 1 part in $10^{9}$, the determination of the gravitational moments will allow us to reach the solar core velocity rate with an unequaled precision.

At last, the solar gravitational moments knowledge can be applied to stars and their planets. The dynamical influence of stellar oblateness may be approximated using the leading order quadrupole terms, neglecting those of order $O(f 2)$.

\footnotetext{
${ }^{5}$ Isaak (1999) called this peering inside the Sun from its surface "a window open to the solar interior"
} 
In the case of an exoplanet of mass $m_{p}$ orbiting around its host star of radius $\mathrm{r}_{*}$ and mass $\mathrm{M}_{*}$, the disturbing part of the stellar potential is thus written as

$$
D=\frac{G M_{*} m_{p}}{2 a_{p}}\left(\frac{R_{*}}{a_{p}}\right)^{2} J_{2}\left(\frac{3}{2} \sin ^{2} i_{p}-1\right)
$$

where $a_{p}$ is the distance of the planet from its host and $i$ its inclination orbit. As an example, the precession rates of planets orbiting the rapidly-rotating main sequence stars WASP-33, Kepler$13 \mathrm{~A}$ and HAT-P-7 reveal associated values of $J_{2, \text { stars }}$ of the order of $10^{4}$.

\section{Annex to subsection "Surfaces of equal potential and level surfaces. Classical mathematical formalism"}

1. Motion for an ideal fluid. The starting point is to consider the equation

$$
\stackrel{\vec{x}}{\rho x}=\rho \vec{g}-\vec{\nabla} p
$$

where $\sim \ddot{x}$ is the acceleration of the fluid, $\rho$ the density, $\sim g$, the force acting on particles, and $\nabla \sim$ $p$, the pressure gradient. For hydrostatic equilibrium, there is no motion, hence $\sim \ddot{x}=0$. For a nonrotating body, the force acting on particles is given by $g=-\nabla \phi_{\text {grav }}$, where $\varphi_{\text {grav }}$ is the gravitational potential. Hence Eq. 27 reduces to

The curl of this equation is $\nabla \rho \mathrm{X} \nabla \phi=0$, so that the normals to surfaces of constant $\rho$ and $\phi$ point in the same direction. As a consequence, these surfaces coincide. Using the perfect gas equation of state (with constant chemical composition), it can be shown that surfaces of constant $\rho, \phi$, temperature $T$ and pressure $P$ all coincide. This is known as the Von Zeipel theorem: any internal source of distortion in the gravitational field at the surface will manifest itself as a change of shape in the solar surface layer. Thus, measuring the shape of the surface layers is equivalent to measuring surfaces of constant gravitational potential.

$$
0=-\rho \vec{\nabla} \phi_{g r a v}-\vec{\nabla} p
$$

When the body is rotating, the force acting on particles is the gradient of the gravity potential $\left(\phi_{\text {total }}=\phi_{\text {grav }}+\phi_{\text {rotation }}\right)$; so we must add the centrifugal force, so that Eq. 28 becomes

$$
\vec{\nabla} p=-\rho \vec{\nabla} \phi_{g r a v}+\zeta^{2}(r, \theta) \vec{s}
$$

where $\zeta(\mathrm{r}, \theta)$ is the angular velocity and $s$ a vector perpendicular to the rotation axis directed outwards.

The equation to a surface of a spheroid whose semi-axes are $R_{e q}$ and $R_{p o l}=R_{e q}(1-f)$ is

$$
\frac{r^{2}(\theta) \cos ^{2} \theta}{R_{e q}^{2}(1-f)^{2}}+\frac{r^{2}(\theta) \sin ^{2} \theta}{R_{e q}^{2}}=1
$$

where $r$ is the radius vector and $\theta$ the colatitude measured from the axis of revolution. Expanding $r(\theta)$ in powers of $f$ it comes 
$r(\theta)=R_{e q}\left[1-f \cos ^{2} \theta-\frac{3}{2} f^{2}\left(\sin ^{2} \theta \cos ^{2} \theta\right)+\frac{1}{8} f^{3}\left(1-5 \sin ^{2} \theta\right) \sin ^{2} 2 \theta+\cdots\right]$

to which corrections can be added to take into account the distorted shape.

\section{Density and pressure surfaces.}

As defined in Armstrong and Kuhn (1999), let $\rho$ the density (function of the radius $r$ ) and denote with a subscript 0 , the lowest order $l$, spherically symmetric. Asphericities, described as

$$
\begin{gathered}
c_{l}=-\frac{\frac{\rho_{l}}{d \rho^{(0)}}}{d r},(\text { density }), \\
s_{l}=-\frac{\frac{p_{l}}{d p^{(0)}}}{d r},(\text { pressure })
\end{gathered}
$$

measure the perturbation (nonspherically symmetric) and are usually expressed in terms of the normalized potential defined by $J_{l}=K \varphi_{l}$, where $K=R / G M$ at the solar surface. The different gravitational moments can be written as

$$
J_{2 l}=\frac{R_{\odot}}{G M_{\odot}} \phi_{2 l}\left(R_{\odot}\right)
$$

where $\phi_{21}=0$ at the surface $r=R$. The function $\phi_{21}$ is the solution to a differential equation requiring the knowledge of $\rho(r)$ and $\omega(\mathrm{r}, \theta)$, where $\theta$ is the colatitude. A complete expression of $\phi_{2}$ and $\phi_{4}$ was provided by Armstrong and Kuhn (1999), using the standard rotation law, which permits to deduce $c_{l}$ :

$$
\begin{gathered}
\frac{d^{2} \Phi_{2}}{d r^{2}}+\frac{2}{r} \frac{d \Phi_{2}}{d r}-6 \frac{\Phi_{2}}{r^{2}} \\
=\frac{4 \pi r^{2}}{M_{r}}\left[\Phi_{2} \frac{d \rho_{0}}{d r}-\frac{8}{21} \frac{2 \omega_{2}}{\omega_{0}} r \rho_{0} \omega_{0}^{2}-\frac{r^{2}}{3} \frac{d}{d r}\left(\rho_{0} \omega_{0}^{2}+\frac{3}{7} \rho_{0} \frac{2 \omega_{2}}{\omega_{0}} \omega_{0}^{2}\right)\right] \\
\frac{d^{2} \Phi_{4}}{d r^{2}}+\frac{2}{r} \frac{d \Phi_{4}}{d r}-\frac{20}{r^{2}} \Phi_{4}=\frac{4 \pi r^{2}}{M_{r}}\left[\Phi_{4} \frac{d \rho_{0}}{d r}+\frac{4}{35} \frac{2 \omega_{2}}{\omega_{0}} r \rho_{0} \omega_{0}^{2}-\frac{2}{35} r^{2} \frac{d}{d r}\left(\frac{2 \omega_{2}}{\omega_{0}} \rho_{0} \omega_{0}^{2}\right)\right]
\end{gathered}
$$

Results are given in Armstrong and Kuhn (1999) showing that the observations are somewhat inconsistent with current rotation models. However, it could be noted noted that a subsurface shear layer results when the helioseismically obtained internal rotation is matched with the surface rotation. Such an approach, using helioseismic data will be made in a second paper. 


\section{References}

Ajabshirizadeh, A., J.P. Rozelot, J.P., Fazel, Z., Contribution of the Solar magnetic field on Gravitational Moments, Scientia Iranica, Vol. 15, No. 1, 144-149 (2008).

Antia, H.M., Chitre, S.M., Gough, D.O., Temporal variations in the Suns rotational kinetic energy. A \& A, 477, 657663 (2008).

Armstrong, J., Kuhn, J.R., Interpreting the solar limb shape distortions. Astrophys. Jour. 525, 533 (1999).

Bertotti, B., Farinella, P., Vokrouhlicky, D., in "Physics of the Solar System. Dynamics and Evolution, Space Physics, and Spacetime Structure", Springer, ed. (2003).

Bois, E., Girard, J.-F., Impact of the quadrupole moment of the Sun on the dynamics of the EarthMoon system, Cel. Mech. 73, 329338 (1999).

Bruns, H., "Die Figur der Erde", Pub. Konigl. Preuss. Geodidat Inst., Berlin (1878).

Cavanaugh, J.F., Smith, J.C., Sun, X., Bartels, A.E., Ramos-Izquierdo, L., Krebs, D.J., McGarry, J.F., Trunzo, R.M., Novo-Gradac, A., Britt, J.L., Karsh, J., Katz, R.B., Lukemire, A.T., Szymkiewicz, R., Berry, D.L., Swinski, J.P., Neumann, G.A., Zuber, M.T., Smith, D.E., Space Sci. Rev. 131, 451 (2007).

Chandrasekhar, S. \& Lebovitz, W., On the oscillations and the stability of rotating gaseous masses., Astrophys. Jour., 136, 1069-1081 (1962).

Chandrasekhar, S., The Equilibrium and the Stability of the Riemann Ellipsoids. I, Astrophys. Jour., 142, 890-921 (1965).

Chandrasekhar, S., The Equilibrium and the Stability of the Riemann Ellipsoids. II, Astrophys. Jour.., 145, 842877 (1966).

Clairaut, A.C., "Thorie de la Figure de la Terre", in Principes de l'Hydrostatique, Durand ed., Paris (1743).

Darwin, G.H., The theory of the figure of the Earth carried to the second order, Monthly Not. Roy. Astro. Soc., 60, 82-124, (1899).

Debras and Chabrier, A complete study of the precision of the concentric MacLaurin spheroid method to calculate Jupiters gravitational moments, A \& A, 609, A97 (2018).

Dicke, R.H., The Oblateness of the Sun and Relativity, Science, 184, Issue 4135, 419-429 (1974).

Dicke, R.H., Kuhn, J.R., Libbrecht, K.G., The variable oblateness of the Sunmeasurements of 1984, Astrophys. Jour. 311, 10251030 (1986).

Emilio, M., Bush, R.I., Kuhn, J., Sherrer, P., A changing solar shape, Astrophys. Jour., 660, L161L163 (2007).

Essen, H., The Physics of Rotational Flattening and the Point Core Model, International Journal of Geosciences, 2014, 5, 555-570 (2014).

Fienga, A., Laskar, J., Kuchynka, P., Manche, H., Desvignes, G., Gastineau, M., Cognard, I., Theureau, G., The INPOP10a planetary ephemeris and its applications in fundamental physics. Celest. Mech. Dyn. Astr. 111, 363385 (2011).

Fienga, A., Laskar, J., Exertier, P., Manche, H., Gastineau, M., Numerical estimation of the sensitivity of INPOP planetary ephemerides to general relativity parameters. Celest. Mech. Dyn. Astr. 123, 325349, (2015).

Fivian, M.D., Hudson, H.S., Lin, R.P., Zahid, H.J., Solar shape measurements from RHESSI: a large excess oblateness, Science 322 (5901), 560562 (2008).

Folkner, W.M., Williams, J.G., Boggs, D.H., Park, R.S., Kuchynka, P., IPN Progress Report 42-196 February 15, 2014. The Planetary and Lunar Ephemerides DE430 and DE431 (2014).

Fossat, E., Boumier, P., et al., Asymptotic g modes: Evidence for a rapid rotation of the solar core, A \& A, 604, 17 (2017).

Godier, S., Rozelot, J.P., A new outlook on the 'Differential Theory' of the solar Quadrupole Moment and Oblateness, Solar Phys., 199, 217-229 (2001).

Hilton, J.L., Hohenkerk, C.Y., A comparison of the high accuracy planetary ephemerides DE421, EPM2008, and INPOP08. Proceedings of the Journees 2010 Systemes de reference spatio-temporels (JSR2010): New challenges for reference systems and numerical standards in astronomy, Observatoire de Paris, 2022 September 2010, Edited by Nicole Capitaine, p. 7780 (2010).

Howard, R., Gilman, P. A., Gilman, P. I., Rotation of the Sun measured from Mount Wilson white-light images, Astrophys. Jour., 283, 373-384 (1984).

Hubbard, W.B., Gravitational field of a rotating planet with a polytropic index of unity, Sov. Astron. Vol. 18, No5 621-625 (1975).

Hubbard, W.B., Effects of differential rotation on the gravitational figures of Jupiter and Saturn, Icarus, 52, 509515 (1982).

Hubbard, W.B., High-precision Maclaurin-based models of rotating liquid planets, Astrophys. Jour. L., 756, L15 (2012). 
Iorio, L., Mercury and frame-dragging in light of the MESSENGER flybys: conflict with general relativity, poor knowledge of the physical properties of the Sun, data reduction artifact, or still insufficient observations? arXiv:1109.0266 (2011).

Isaak, G.R., Solar core rotation, in European Meeting on Solar Physics, 12-18 September 1999, Florence (I), ESA SP-448 (1999).

Jacobi, C. G. J., De usu legitimo formulae summatoriae Maclaurinianae, Journal fur die Reine und Angewandte Mathematik, Volume 1834: Issue 12, 263-281 (1834).

James, R. A., The structure and stability of rotating gas masses, Astrophys. Jour., 140, 552-582 (1964).

Javaraiah, J. and Komm, R.W.: "Sun's Rotation", Nova Science Publishers, Inc., Javaraiah, J. and Gokhale, M.H. eds. (2002).

Javaraiah, J., Rozelot, J.P., technical papers CERGA observatory (2002).

Jeans, J. H., Gravitational instability and the nebular hypothesis, Phil. Trans. Roy. Soc. London, 213, 457-485 (1914).

Klioner S. A., A Practical Relativistic Model for Microarcsecond Astrometry in Space, Astronom. Jour., 125, 1580-1597 (2003).

Komm, R., Howe, R., Durney, B.R., Hill, F., Temporal Variation of Angular Momentum in the Solar Convection Zone, Astrophys. Jour., 586, 650-662 (2003).

Kuiper, J., in "The Sun”, Chicago University Press (USA) (1972).

Lefebvre, S., Kosovichev, A.K., Rozelot, J.P., Helioseismic Test of Nonhomologous Solar Radius Changes with the 11 Year Activity Cycle, Astrophys. Jour., 658, L135-L138 (2007).

Lydon, T.J., Soa,S., XXX, Phys. Rev. Lett. 76, 177 (1996).

Lyttleton, R.A., The Stability of Rotating Liquid Masses, Cambridge University Press (1953).

Maclaurin, C., in Thomson, W. \& Tait, P. G., Treatise on Natural Philosophy, Cambridge University Press, Part II, Art. 772 (1742).

Maeder, A., Stellar evolution with rotation IV: von Zeipels theorem and anisotropic losses of mass and angular momentum, A \& A, 347, 185-193 (1999).

Milne, E.A., The equilibrium of a rotating star, Monthly Notices Royal Astron. Soc., 83, 119-147 (1923).

Modolenvsky, M.S., XXX, Geodezika i Kartografiya, 5, 11 (1988).

Moritz, H., "The Figure of the Earth", Wichmann ed. Paris (1980).

Newcomb, S., Constants of Astronomy, in: "The Elements of the Four Inner Planets and the Fundamental Constants of Astronomy" US GPO, Washington, DC (USA), p. 111 (1865).

Ostriker, J.P., Mark, J.W.K., Rapidly rotating stars. I. The self-consistent-field method, Astrophys. Jour., 151, 1075-1088 (1968).

Panhans M., Soffel M. H., Gravito-magnetism of an extended celestial body, Classical Quant. Grav., 31, 245012 (2014).

Park, R.S., Folkner, W.M., Konopliv, A.S., Williams, J.G., Smith, D.E., Zuber, M.T., Precession of Mercurys Perihelion from Ranging to the MESSENGER spacecraft. Astronom. Jour. 153 (3), 121 (2017).

Paterno, L., Sofia, S., DiMauro, M.P., The rotation of the Suns core. A \& A 314, 940946 (1996).

Pijpers (1998) Pijpers, F.P., Helioseismic determination of the solar gravitational quadrupole moment, Monthly Notices Roy. Soc. 297, L76L80 (1998).

Pireaux, S., Signature dobservables en th'eories alternatives de la gravitation. Relativite Generale et Cosmologie Quantique. Universit'e Catholique de Louvain, (2002).

Pireaux, S., Rozelot, J.P., Solar quadrupole moment and purely relativistic gravitation contributions to Mercurys perihelion advance, Astrophys. Space Sci. 284, 11591194 (2003).

Pireaux, S., Lefebvre, S., Rozelot, J.P., Solar gravitational moments and solar core dynamics, SF2A-2005: Semaine de l'Astrophysique Fran, caise, meeting held in Strasbourg, France, June 27-July 1, 2005, Edited by F. Casoli, T. Contini, J.M. Hameury and L. Pagani. Published by EdP-Sciences, Conference Series, 2005, p. 121 (2005).

Pitjeva, E.V., Relativistic effects and solar oblateness from radar observations of planets and spacecraft, Astron. Lett. 31 (5), 340349 (2005).

Pitjeva, E.V., Updated IAA RAS Planetary Ephemerides-EPM2011 and their use in scientic research. Solar System Research, Vol. 47, No. 5, pp. 386402. Original Russian Text published in Astronomicheskii Vestnik, 2013 , Vol. 47, No. 5, pp. 419435 (2013).

Pitjeva, E.V., Pitjev, N.P., Development of planetary ephemerides EPM and their applications, Celest. Mech. Dyn. Astr. 119 (3), 237256 (2014).

Poincare, H., in "Tisserand, F., Mcanique Celeste”, Gauthier-Villars ed., Paris, 2, chap. xi (1891).

Radau, R., "Remarques sur la Theorie de la Figure de la Terre", Bull. Astron., 2, 157 (1885).

Riemann, B., Abb. d. Konigl. Gessel, der Wis. d. Gottingen, 9, 3-36 (1860). 
Rieutord, M., Espinosa Lara, F., \& Putigny, B. J., Problems raised by (fast) rotating stars, Comput. Phys., 318, 277 (2016).

Rozelot and Rosch, An Upper Bound to the Solar Oblateness, Sol. Phys., 11-18 (1997).

Rosch, J., Rozelot, J.P., Le Soleil change-t-il de forme?, Comptes Rendus Acad. Sci. Paris serie II, 322637644 (1996).

Rozelot, J.P., Lefebvre, S., Desnoux, V., Solar limb shape distortions, Solar Phys. 217, 3952 (2003).

Rozelot, J.P., Bois, E., New results concerning the solar oblateness and consequences on the solar interior. In: Balasubramaniam, K.S., Rabin, D.M. (Eds.), 18th NSO Workshop, Sacramento Peak, USA, Conference of the Pacic Astronomical Society, no. 140, pp. 7582 (1997).

Rozelot, J.P., Lefebvre, S., The Figure of the Sun, astrophysical consequences. In: "The Sun's Surface and subsurface, Investigating Shape and Irradiance", Lecture Notes in Physics, vol. 599, Springer, Heidelberg (D), pp. 427 (2003).

Rozelot, J.P., Damiani, C., Pireaux, S., Probing the solar surface: the oblateness and astrophysical consequences, Astrophys. Jour. 703, 17911796 (2009).

Rozelot, J.P., Fazel, Z., Revisiting the Solar Oblateness: Is Relevant Astrophysics Possible? Sol. Phys., 287, 161170 (2013).

Rozelot, J.P., Eren, S., Exploring the temporal variation of the solar quadrupole moment from relativistic gravitation contributions: A fortuitous circumstance? Ad. Sp. Research, 65, 2821-2827 (2020).

Rozelot, J.P., Kosovichev, A.K., Kilcik, A., Solar oblateness \& asphericities temporal variations: outstanding some unsolved issues, in "Solar and Stellar Magnetic Fields: Origins and Manifestations", IUA symposium 354, A.G. Kosovichev, K. Strassmeier \& M. Jardine, eds., 232-237 (2020).

Rumiantsev, V.V., The stability of Maclaurin ellipsoids of a rotating fluid, PMM Vol.23, No.3, 494-504, (1959).

Scheiner, C., "Rosa Ursina sive sol ex admirando facularum (...)" (1630).

Scherrer, P. H., Bogart, R. S., Bush, R. I., Hoeksema, J. T., Kosovichev, A. G., Schou, J., Rosenberg, W., Springer, L., Tarbell, T. D., Title, A., Wolfson, C. J., Zayer, I., MDI Engineering Team, The solar oscillations investigation - Michelson Doppler Imager, Solar Phys., 162, 129-188 (1995).

Spalding, C., Millholland, S.C., Stellar Oblateness versus Distant Giants in Exciting Kepler Planet Mutual Inclinations, Astronomical Journal, 160, Issue 3, id.105 (2020).

Standish, M., JPL Interoffice Memoradum, IOM 312.F - 98-048, August 26, 1998, 18pp.(1998).

Stoeckly, R., Polytropic Models with Fast, Non-Uniform Rotation, Astrophys. Jour., 142, 208-228 (1965).

Turck-Chieze, Palacios, A., Marques, J. P., Nghiem, P. A. P.S., Seismic and Dynamical Solar Models. I. The Impact of the Solar Rotation History on Neutrinos and Seismic Indicators, Astrophys. Jour., 715, 1539-1555 (2010).

Verma, A.K., Fienga, A., Laskar, J., Manche, H., Gastineau, M., Use of MESSENGER radioscience data to improve planetary Ephemeris and to test general relativity. Astron. Astrophys. 561, A115 (2014).

Wavre, R., "Figures plantaires et godsie", Gauthiers-Villars ed., Paris (1932).

Williams J. G., Turyshev, S. G., Slava, G., Boggs, Lunar Laser Ranging Tests of the Equivalence Principle with the Earth and Moon, D. H., Int. J. Mod. Phys. D, 18, 1129-1175 (2009).

Xu, Y., Shen, Y., Xu, G., Shan, X., Rozelot, J.P., Perihelion precession caused by solar oblateness variation in equatorial and ecliptic coordinate systems, Monthly Notices Roy. Soc., 472, 26862693 (2017).

Xu, Y., Xu Y., Yang Y., Zhang Q., Xu G., Solar oblateness and Mercury’s perihelion precession, Monthly Notices Roy. Soc., 415, 3335-33 (2011).

Yuan, D-N., Sjogren, W.L., Konopliv, A.S., Kucinskas, A.B., J. Geophys. Res. E, 106, 23377 (2002).

Zharkov, V.N. \& Trubitsyn, Physics of Planetary Interiors, Pachart ed., Arizona Press (USA) (1978). 


\title{
On Superflares on the Sun and Stars
}

\author{
Katsova M.M. ${ }^{1}$, Obridko V.N. ${ }^{2}$, Sokoloff D.D. ${ }^{2,3,4}$, Livshits I.M. ${ }^{1}$ \\ ${ }^{1}$ Sternberg State Astronomical Institute, Lomonosov Moscow State University, Moscow, \\ Russia \\ ${ }^{2}$ Pushkov Institute of Terrestrial Magnetism, Ionosphere and Radiowave Propagation of \\ Russian Acadmy of Sciences, Troitsk, Moscow, Russia \\ ${ }^{3}$ Faculty of Physics, Lomonosov Moscow State University, Moscow, Russia \\ ${ }^{4}$ Moscow Center of Fundamental and Applied Mathematics, Moscow, Russia \\ E-mail: mkatsova@mail.ru
}

\begin{abstract}
.
We discuss the current observations of the most powerful non-steady phenomena on solarlike stars. While remaining within even the most extreme solar ideas, there is problematic to get the flare energy more than (3-5) $\times 10^{34} \mathrm{erg}$, which is apparently an absolute upper limit for solar-type flares. For explanation of the higher flare energy, about of $10^{36} \mathrm{erg}$, one need to adopt that spots with the magnetic field strength of a few $\mathrm{kG}$ should cover more than $30 \%$ of a hemisphere. This estimate leads to a mean magnetic field around $1 \mathrm{kG}$. New observational evidences for a presence of the strong relict magnetic fields on solar-like stars appeared recently. We discuss to what extent it is necessary to change the mechanisms of convection and dynamo with a corresponding change in the models of the atmosphere. We consider possible ways of solving the problem of the energy of superflares.
\end{abstract}

\section{Introduction}

As it is known, the total energies of solar flares from weakest events to the strongest ones span in the wide range $10^{24}-10^{32} \mathrm{erg}$. Stellar flares are best studied on low-mass, red dwarf stars, and their total energies exceed the maximal solar value by several orders of magnitudes. Besides, the most powerful of these flare phenomena were mainly registered on very young active stars (pre-main-sequence $\mathrm{T}$ Tau-type stars, members of the young open clusters), on chromospherically active RS CVn-type stars that are components of close binary systems as well. The total energies of the strongest of stellar flares there can reach values of $\geq 10^{36} \mathrm{erg}$ in the optic waveband. As for flare activity of solar-like single $\mathrm{G}$ dwarf stars, there was very little information prior the Kepler mission.

The superflare concept applicable to powerful non-stationary stellar phenomena has been introduced when the first results of the Kepler mission operated in 2009-2018 with a detection of huge flares on G-type stars were published [Maehara et al. 2012, 2015]. They reported on registration of large stellar flares on solar-like stars with the total energies from $10^{33}$ to $>10^{37}$ ergs at optical wavelengths. The more detail analysis showed that the most of flares have the total energies $10^{33}-10^{34} \mathrm{erg}$, while only a small fraction of the phenomena can be considered as superflares with the energies $\sim 10^{35}-10^{36}$ эрг. Later on it became clear that the most powerful events with $\mathrm{E}>10^{36} \mathrm{erg}$ occur either on components of close binary stellar systems or on giants and subgiants or on very young stars that have not reached the main sequence [Katsova and Nizamov, 2018].

An analysis of multiwavelength observations of stellar flares and other non-steady phenomena on red dwarfs and solar-like stars gives evidences for their common physical nature to solar ones [Gershberg 2005, Gershberg et al. 1987, 2020]. This is about a deposit of the free energy of the non-potential magnetic field in a certain volume, its impulsive release during nonsteady process, a subsequent response of the atmosphere to arising acceleration particles and plasma heating. At the same time, already Gershberg et al. [1987] paid attention to the unsatisfactory of modern models of solar flares for explanation the strongest stellar flares. 


\section{An accumulation of the free energy for the generation of solar flares}

Traditionally, the total energy E, required for a flare can be calculated as follows $E=f B^{2} L^{3} / 8 \pi$ Essentially, it is supposed that the magnetic field is enclosed in a volume with characteristic dimensions $\boldsymbol{L}$, and it is constant there. The factor $\boldsymbol{f}$ shows that the only nonpotential fraction of the field dissipates. Assuming that $\boldsymbol{B}=3000 \mathrm{G}$ and $\boldsymbol{L}=3 \times 10^{9} \mathrm{~cm}$, we get $\sim 3 \times 10^{32} \mathrm{erg}$. This value is close to the energy evaluation for the most powerful solar flares and this situation could be satisfactory. However, some parameters in these calculations are overstated and, in any case, significantly differ from statistical properties of the largest sunspots.

\section{Evaluations of the magnetic field strength and the structure in sunspots at the photosphere level}

The sunspot is a area of an enhanced strength of the magnetic field. However, so far there is no reliable data on the magnetic field strength on a boundary of the spot. This value is very important, because namely it determines a decrease of the convective heating of the photosphere occurs this as well as an appearance of the dark formation. It is difficult to measure this value due to the irregularity of boundaries and difficulties in drawing isolines. This is even more difficult to do this on high-resolution magnetograms. Therefore, we proposed a new approach - calculation of area in which the field is above a certain threshold [Obridko and Shelting, 2018]. Then the threshold value at which the total "magnetic" area coincides with the total spot area measured on that day can be called the spot boundary.

We used daily SDO/HMI data on a longitudinal component of the magnetic field during 2375 days from 01. May 2010 to 31. October 2016. Daily sunspot number data were taken from the site WDC-SILSO, Royal Observatory of Belgium, Brussels http://sidc.oma.be/silso/datafiles (version 2). The total daily values of sunspot areas were adopted in NASA site https://solarscience.msfc.nasa.gov/greenwch.shtml.

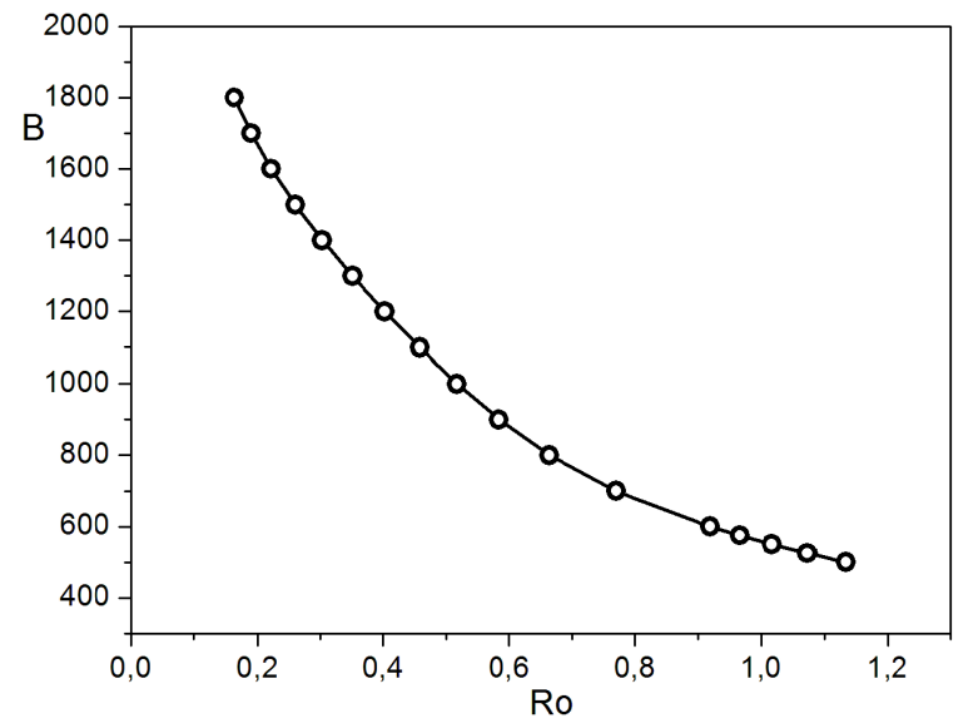

Fig.1. The magnetic field strength, $\boldsymbol{B}$, versus the relative distance in the spot, covered by the field above the threshold value in real spots, $\boldsymbol{R o}$.

It turned out that the spot boundary corresponds to the magnetic field value of $550 \mathrm{G}$. The stronger magnetic fields above $2000 \mathrm{G}$ occupy an extremely small part of the spot, no more than $20 \%$. The field falls off rather quickly with distance from the center of the spot (Fig.1). 


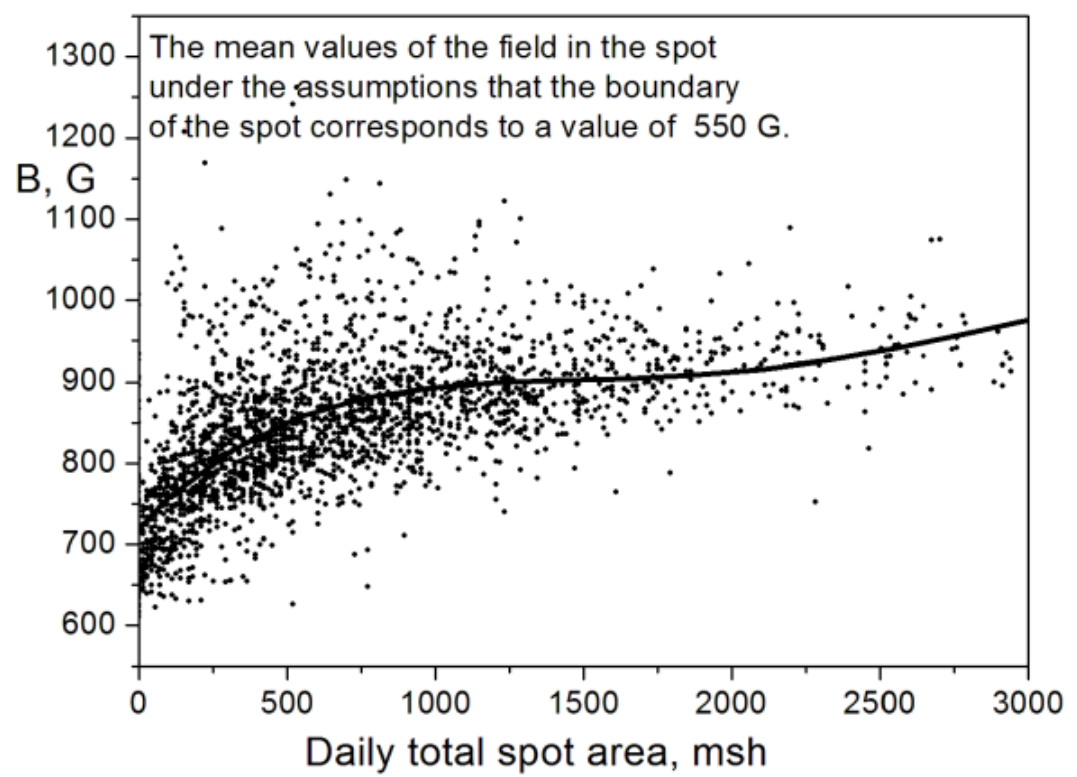

Fig.2. The mean magnetic field strength values in spots.

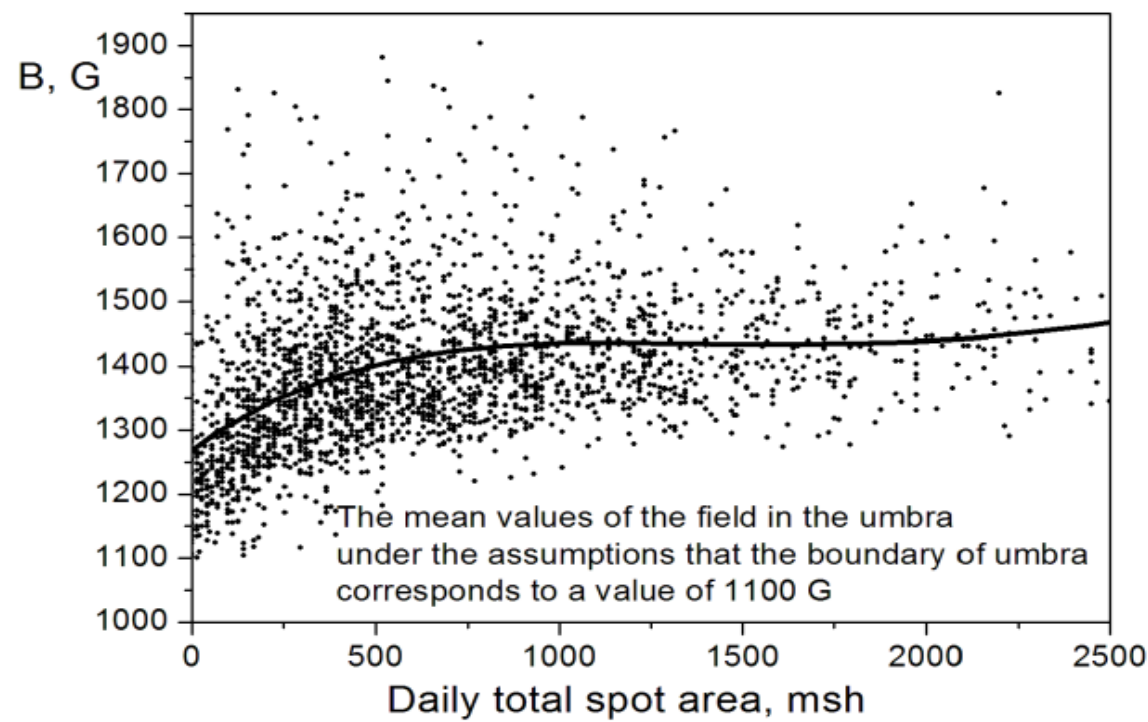

Fig.3. The mean values of the field in the umbra The line presents the approximation by the 4th degree polynomial.

The mean value $\boldsymbol{B}$ is around $750 \mathrm{G}$ for the smallest spots, it increases slightly up to $900 \mathrm{G}$ in those days, when the total spot area on the Sun is 500-2000 msh, and then grows up a little more. This value seems rather small, but it should be taken into account that the main contribution here comes from penumbra, which occupies about $80 \%$ of the sunspot area [Bludova et al. 2014]. It is not easy to highlight here the average field values in the total umbrae of the spots, since the magnetic boundary of the umbra is even more undefined than the boundary of the umbra. The photometric boundary of the umbra (i.e., the inner boundary of the penumbra) is usually more blurred than its outer one. Based on the relative umbra area, it can be assumed that the boundary of the umbra corresponds to the magnetic field strength of 1000$1100 \mathrm{G}$. The mean field values in the spot umbrae under such assumptions are shown in Fig. 3. 
Thus, the average field values in the sunspot umbra are $1400 \mathrm{G}$ and also weakly depend on the area. These values obtained look rather low compared to the average value given in [Obridko and Nagovitsyn, 2017], which was $2050 \mathrm{G}$, and it was obtained from the results of the 1957-1997 database http://www.gao.spb.ru/database/mtbase/. It should be noted, however, that the purpose of these observations was mainly to find the maximum field value for each spot.

In this case, the relative fraction of the area of the umbra is about $20 \%$ and very weakly decreases with the spot area (Fig. 4).

It is much more difficult to estimate the height at which the reconnection zone $\mathbf{L z}$ is located. According to various estimates, this should be the lower corona with a density $\boldsymbol{n}_{e^{\sim}} 3 \times 10^{8} \mathrm{~cm}^{-3}$ or somewhat more. The geometrical height of this area can vary significantly. It is higher in undisturbed region $(5-7$ thousand $\mathrm{km})$ and is lowered over the spot to values of the order of 1-2 thousand $\mathrm{km}$. Some observational values can be used to estimate the magnetic field at this altitude (see Obridko, 1985, and references therein). The field gradient falling with height may be less than $0.7 \mathrm{G} / \mathrm{km}$ at different spots. This means that one can expect the field of about $1000 \mathrm{G}$ at an altitude of 1-2 thousand $\mathrm{km}$ above the sunspot.

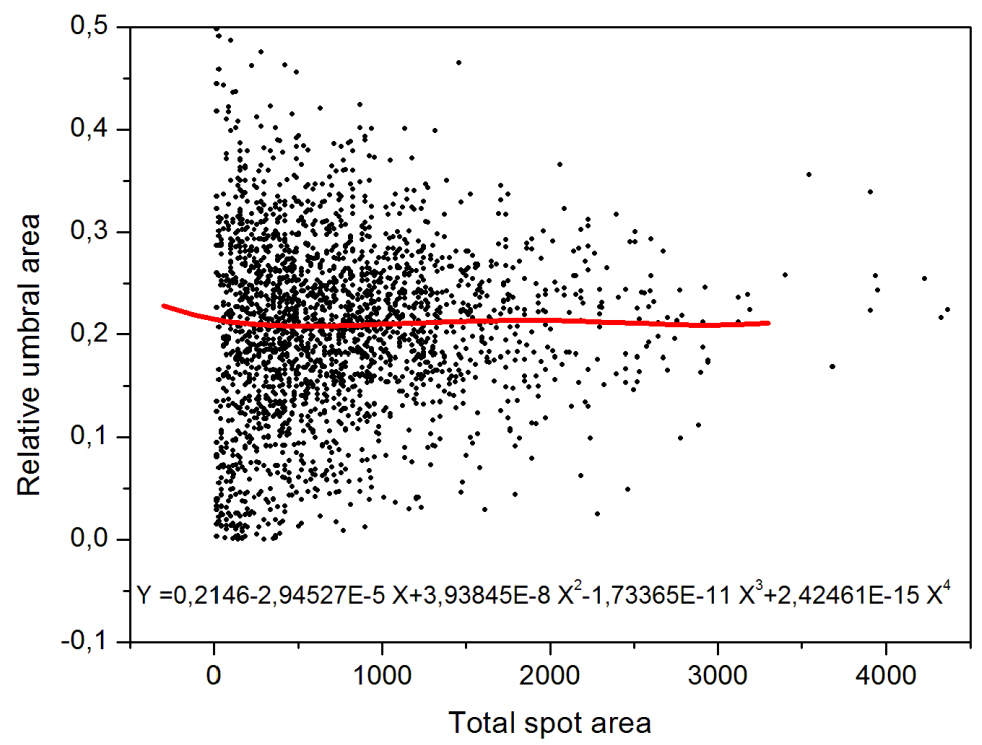

Fig.4. The relative fraction of the umbra area versus the total spot area, msh

Thus, evaluating the energy stored in the flare region, should be calculated on a more complicated formula $\boldsymbol{E}=\boldsymbol{S} \boldsymbol{L}_{z} \boldsymbol{B}^{2} / \mathbf{8} \pi$, where $\boldsymbol{S}$ is a flare area, $\boldsymbol{B}$ is a volume-averaged field strength. For the above values in a very large spot (although not the largest one) with an area of $1000 \mathrm{msh}$ and an average field value of $1400 \mathrm{G}$ and a height of $\boldsymbol{L}_{z}=10^{8} \mathrm{~cm}$, we obtain $2.4 \times 10^{32} \mathrm{erg}$, which is also close to the observed values for the most powerful solar flares.

However, it should be noted that our calculations were guided by the averages rather than the maximum values observed in the past. Let us now estimate the energy that would correspond to the simultaneous appearance of all extreme parameters. The largest spots reach an area of $4000 \mathrm{msh}$. For the entire time of observations of the magnetic fields of the spots, 55 active regions were registered, with a magnetic field above $4000 \mathrm{G}$. Note that these "superfields" occupied an extremely small effective area in active regions. Finally, recently, based on observations of SDO/HMI and Hinode SOT/SP by calculations of a force-free magnetic field, a magnetic field of $4000 \mathrm{G}$ was found at an altitude $10^{8} \mathrm{~cm}$ [Afinogentov et al. 2019]. Assuming that the average field is $4000 \mathrm{G}$ over the entire area of $4000 \mathrm{msh}$, we get 
$\boldsymbol{E}=8 \times 10^{33} \mathrm{erg}$. This value can be considered as the absolute upper limit for the energy of solar flares.

\section{On the energies of stellar superflares}

Let us consider the possibility of accumulating energy sufficient for stellar superflares with energies up to $10^{36} \mathrm{erg}$. In this case, we will be guided by the already used concept of the similarity of flares on the Sun and stars, mentioned at the beginning of the article, and the second expression for the energy in the previous section. It is necessary to change the parameters of the star so as to obtain $\boldsymbol{E}=10^{36} \mathrm{erg}$. Since we assume that we are dealing with a solar-like star, the characteristics of the corona should be the same. As a consequence, the dependence of the density in the corona should be preserved and, accordingly, the value of $\boldsymbol{B}_{z}$ changes a little. The most promising is to increase the area. But, it is impossible to increase the area by 4 orders of magnitude, the average area of a large spot $(300 \mathrm{msh})$ limits our capabilities. If we suppose an increase in 1000 times, while the spotted area should be 0.3 of the hemisphere area. Then we get the energy of $3 \times 10^{35} \mathrm{erg}$, which in principle is already enough. The rest can be added by a slight $10 \%$ increase in the remaining parameters.

However, the difficulties/problems did not disappear, but moved to another plane.

In fact, the mean magnetic field at such a star will be $1-2 \mathrm{kG}$, which is not observed. There is no way to avoid this sad conclusion. You can try to increase the $\boldsymbol{L}_{z}$, but it's not much help. An increase in $\boldsymbol{L}_{z}$ means that the flare occurs at heights of a few tenths of the star's radius. Anything strong magnetic field at this height is impossible. In addition, such star should exhibit considerable variability in the magnetic field strength with amplitude 800-1200 G, brightness $-10 \%$ and several times in EUV - and microwave range. A spot of such a huge size should lead to a decrease in the average brightness by $20-25 \%$ and a certain change in the spectral type towards the K-type. A region of strong overheating should appear under such a spot. There is such an area under the sunspot, but there heat diffuses into the lateral regions and comes out to the surface in the form of a light ring surrounding the sunspot. There will be nowhere to diffuse. In the subsurface layers, convection will be stopped over a large area. It is not yet clear, how the dynamo will operate under such conditions and whether it is compatible with the generation of strong magnetic fields. However, it is possible that recent observations of magnetic fields on the young solar twins [Kochukhov et al. 2010] can be understood as an indication of the possibility of just such a stellar dynamo operation. Apparently, such a dynamo must operate directly on the surface of the star, so that a hypothetical spot, occupying almost the entire surface of the star, should be continuously regenerated by the dynamo mechanism. A powerful coronal condensation should appear over such a giant spot, and such stars should have very strong ultraviolet, X-ray and radio emission.

The duration of the solar and stellar flares could not fit on one line. These distributions of solar and stellar flares cannot be explained by the same power-law relationship, and the durations of superflares are an order of magnitude shorter than those extrapolated from the power.

Thus formulated more than 30 years ago [Gershberg et al. 1987], the difficulties not only failed to overcome, but with the availability of new data matching problem of solar flares and stellar superflares become even more acute. Despite the similarity of general physical manifestations, it must be admitted that there are significant differences either in the mechanisms of magnetic field generation, or in the main flare model, or in both.

These results were obtained with the partial support by Russian Foundation of Basic Researches, project No 19-02-00191. 


\section{References}

Anfinogentov, S., Stupishin, A.G., Mysh'yakov, I.I., Fleishman G.D. (2019). Record-breaking Coronal Magnetic Field in Solar Active Region 12673, Astrophys. J. Let. 2019, 880, Issue 2, article id. L29, 5 pp.

Bludova, N.G., Obridko, V.N., Badalyan, O.G. (2014). The Relative Umbral Area in Spot Groups as an Index of Cyclic Variation of Solar Activity, Solar Physics, 289, Issue 3, 1013-1028.

Gershberg, R.E., Kleeorin, N. I., Pustilnik, L.A., Shlyapnikov, A.A. (2020). Физика звёзд средних и малых масс с активностью солнечного типа. Москва, Физматлит. 768 с.

Gershberg, R.E., Mogilevskii, E.I., Obridko, V.N. (1987). Energetics of activity of flare stars and the Sun: a synergetical approach, Kinematika Fiz. Nebesn. Tel. (Кинематика и физика небесных тел), 3 No 5, 3-17 (in Russian).

Katsova, M.M., Nizamov, B.A. (2018). Properties of Kepler Stars with the Most Powerful Flares, Geomagnetism \& Aeronomy, 58, No 7, 899-904.

Kochukhov, O, Hackman, T., Lehtinen, J.J., Wehrhahn, A. (2020). Hidden magnetic fields of young suns, Astron. Astrophys., 635, id. A142, 23 pp.

Maehara, H., Shibayama, T., Notsu, S., et al. (2012). Superflare on solar-type stars, Nature, 485, Issue 7399, 478.

Maehara, H., Shibayama, T., Notsu, Y., et al. (2015). Statistical properties of superflares on solar-type stars based on 1-min cadence data, Earth, Planet \& Space, 67, 59.

Obridko, V.N. (1985) Солнечные пятна, комплексы активности, 1985, Москва, Наука.

Obridko, V.N., Nagovitsyn, Yu.A. (2017). Солнечная активность, цикличность и методы прогноза. с.248, СПб, ВВМ.

Obridko, V.N., Shelting, B.D. (2018). Some Statistical Properties of Magnetic Fields and Sunspots, Research Notes of the American Astronomical Society, 2, Issue 1, article id. 40 (March 13) DOI: 10.3847/25155172/aab55c. 


\title{
Morphological and Kinematical Study of Causally- Linked Filament Eruptions
}

\author{
Koleva K. ${ }^{1}$, Chandra R. ${ }^{2}$, Joshi $R^{2}{ }^{2}$ Devi $P^{2}{ }^{2}$, Duchlev $P .^{3}$, Dechev $M .^{3}$ \\ ${ }^{1}$ Space Research and Technology Institute, Bulgarian Academy of Sciences \\ ${ }^{2}$ Department of Physics, DSB Campus, Kumaun University, Nainital - 263 002, India \\ ${ }^{3}$ Institute of Astronomy and NAO, Bulgarian Academy of Sciences
}

\section{E-mail: kkoleva@space.bas.bg}

\begin{abstract}
In this study we present the multi-wavelength observations of three linked filament eruptions occurring on 2015 July 19. The events were observed in UV/EUV wavelengths by Atmospheric Imaging Assembly (AIA) onboard the Solar Dynamics Observatory (SDO) and GONG in H-alpha wavelength. The third filament eruption was associated with GOES C2.1 class flare and coronal mass ejection (CME). These three filament eruptions are successive and sympathetic in nature. Using the time-distance analysis technique, we have discussed the kinematics of the erupting filaments.
\end{abstract}

\section{Introduction}

Eruptive prominences (EPs) (or filaments if observed on the solar disk) are large-scale phenomena occurring in the solar atmosphere. They are frequently associated and physically related to coronal mass ejections (CMEs) and flares (Tandberg-Hanssen, 1995; Munro et al., 1979; Chandra et al., 2017). Such close relationship between EPs and the other eruptive solar occurrences (Lin et al, 2003; Priest and Forbes, 2002) suggests that the three eruptive events are different manifestations of a same huge physical process, whose energy source is the free energy stored in coronal magnetic fields (Forbes, 2000). Observations show that prominences display a wide range of eruptive activity. For example, according to observational definition of Gilbert et al. (2007) three basic types of the filaments/prominences eruptions exist, based on the relation between the filament mass and the corresponding supporting magnetic structure: full, partial, and failed.

Among the variety of solar eruptions there are cases when two and more filaments erupt in a close temporal and spatial correlation with linkage between them. Such eruptions are so-called "sympathetic" eruptions. Sympathetic filament eruptions are defined as consecutive linked eruptions occurring within a relatively short time period in different but physically related locations (Moon et al., 2002; Yang et al., 2012; Joshi et al., 2016; Wang et al., 2016). Observational and numerical research point that the physical linkages between the sympathetic eruptions have a magnetic nature (Ding et al., 2006; Wang et al., 2007; Jiang et al., 2008; Török et al., 2011; Wang et al., 2016). Török et al. (2011) did 3-dimensional MHD simulations of sympathetic eruptions, observed on 2011 October 01, and suggested two possible magnetic trigger mechanism. One possibility is the presence of a separator above closed flux system and second possibility is the presence of an adjacent closed flux system overlying a flux rope.

In this work we present the study of the successive sympathetic eruptions of three quiet filaments, namely F1, F2 and F3, that occurred on 2015 July 19 in the western solar hemisphere. The used data, morphology and events timing are described in Section 2. The kinematics is presented in Sections 3. Finally the summary is given in Section 4.

\section{Observations}

\subsection{Data Sets}


In the present work we used high spatial and temporal resolution data in Extreme Ultraviolet (EUV) channels from the Atmospheric Imaging Assembly (AIA; Lemen et al., 2012) on board the Solar Dynamics Observatory (SDO, Pesnell et al., 2012). The filament eruption kinematics and morphology are studied by data in He II $304 \AA$ channel of AIA/SDO. H-alpha images from Global Oscillation Network Group (GONG; Harvey et al. 2011) were used to investigate the location and chromosphere nature of filament eruption. In order to search for a possible association with CMEs, data from LASCO/SoHO CME catalog were also used: https://cdaw.gsfc.nasa.gov/CME_list/(Gopalswamy et al., 2009).

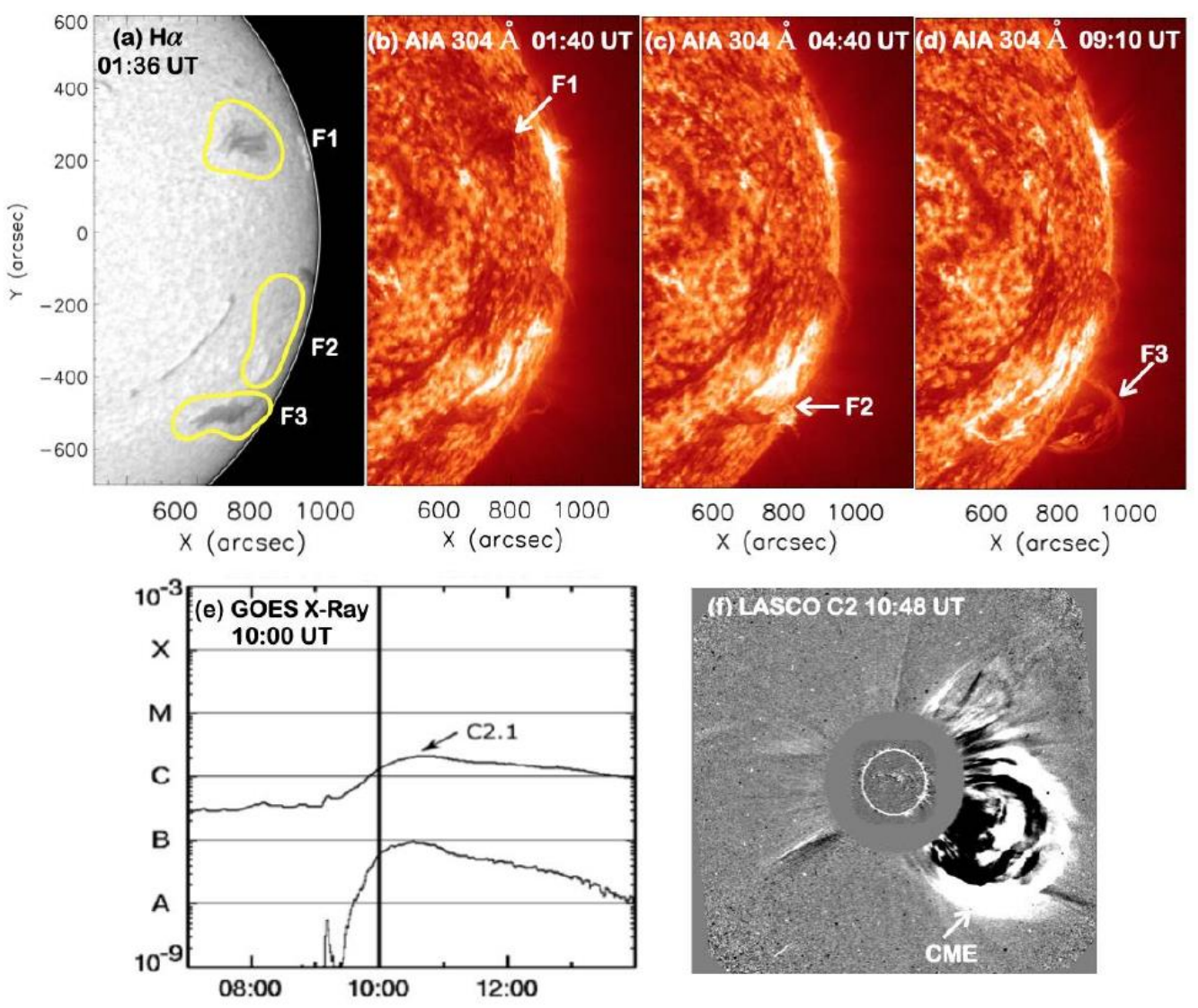

Figure 1: (a) Yellow contours indicate the location of filament F1, F2 and F3 before the eruption on $\mathrm{H}$ alpha image. $(b-d)$ AIA $304 \AA$ images during the three filament eruption. $(e, d)$ GOES flare and $C M E$ associated with F3 filament eruption.

\subsection{Morphology and events timing}

On 2015 July 19, we have observed the successive eruptions of three quiet filaments from the western hemisphere. Afterwards, we will refer them as F1, F2 and F3 respectively. Based on the eruptions evolution and the closeness in time of the erupting filaments, we can interpret them as sympathetic eruptions. The location of filaments before the eruptionis is labeled in Figure 1a.

Initially the quiet filament F1 located in northern hemisphere stared to erupt around 01:00 UT. We found some part of the filament was erupted mostly radiallyin the north-west direction. However, the eruption could not evolved into CME. Together with this eruption some part of the filament was observed to fall back towards the south direction, where F2 filament was located. Due to the fall back of this material, the filament F2 located in southern hemisphere becomes unstable and starts to erupt in west-south direction. Afterward some part of this filament falls down to the solar surface. The downfall material of the filament $\mathrm{F} 2$ again triggered another filament F3, which is located very close to the filament F2 (about 50 arcsec). As a result 
of this trigger, the filament F3 started to erupt and the filament material is gone in the west direction. The F3 eruption produced a pair of long flare ribbons, which became a gradual GOES C2.1 class flare. The Figure 1(b-d) shows the images of filament eruption in AIA 304 A.

Further the eruption of filament F3 was associated with a partial halo CME, observed by the Large Angle and Spectrometric Coronagraphs (LASCO) aboard SOHO. The angular width and the speed of the CME are $194 \mathrm{deg}$ and $782 \mathrm{~km} / \mathrm{s}$, respectively. The morphological evolution of these eruptions is displayed in Figure 1 in $\mathrm{H}$-alpha and UV/EUV wavelengths. The associated GOES flare and the CME are also depicted in same figure.

\section{Eruption Kinematics}

To explore the temporal relation between these sympathetic eruptions, we tracked the eruption kinematics using the slice-time plots. For this, we have selected three artificial slices viz. S1, S2 and S3 respectively. The direction of the slices are selected according to the direction of filament material ejection. The location of slices and the results of this analysis as a timedistance plots are shown in Figure 2 and 3 respectively.
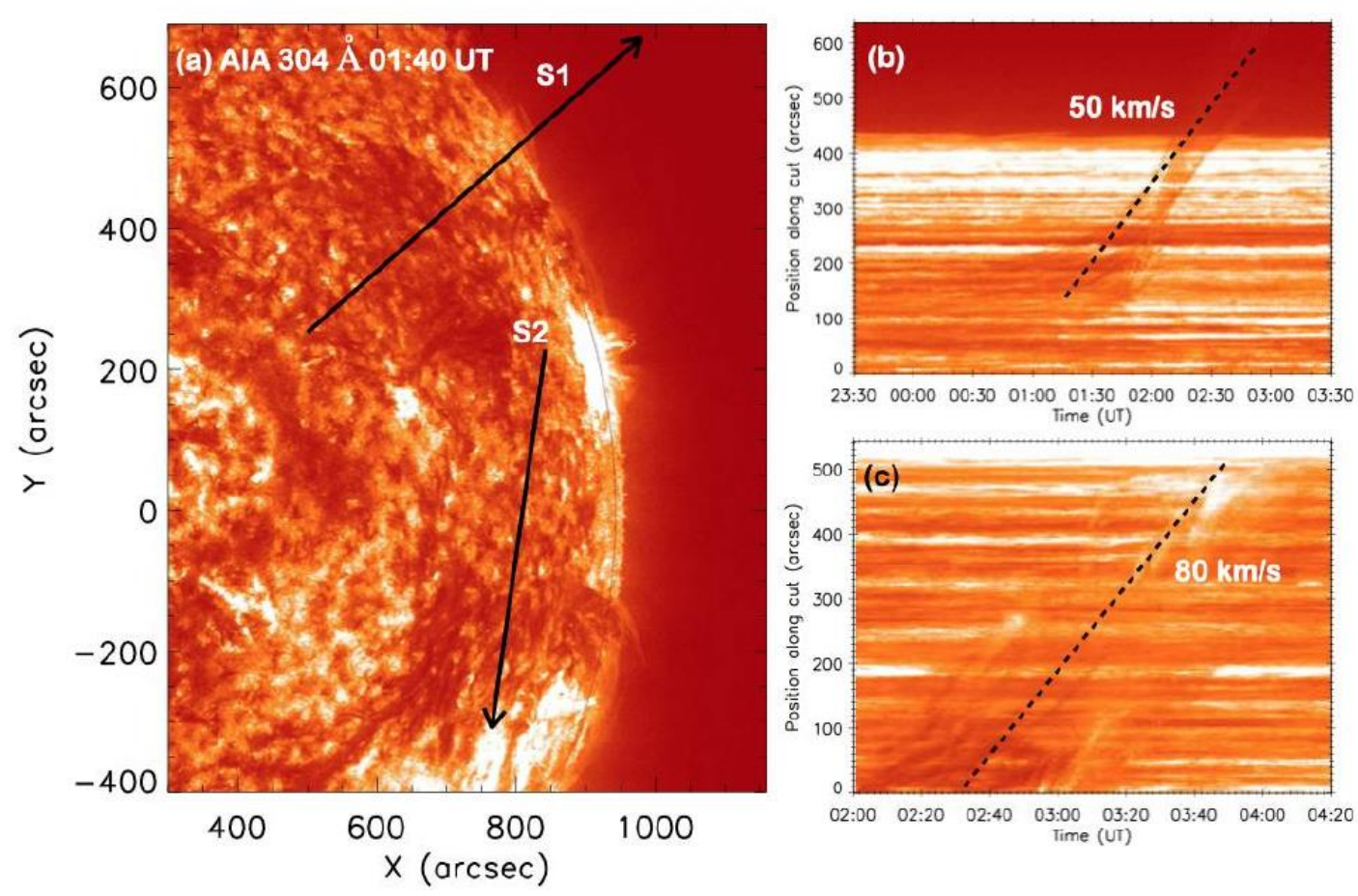

Figure 2: (a) Location of the two slices selected for the height-time plot of F1 eruption in North-West direction and the filament material flow in south direction. $(b, c)$ The height-distance plot along the slits $S 1$ and $S 2$ respectively.

Eruption of F1 stared around 01:00 UT in the west direction as well as some material erupted in south direction. We have computed the speed of eruption in both the direction. The observed speed in north-west direction $50 \mathrm{~km} / \mathrm{s}$. While the observed speed in south direction computed along the slice S2 direction (see Figure 3) is about $80 \mathrm{~km} / \mathrm{s}$. From the time-distance plot and movie also, we found that the erupting material reached close to the location of filament F2 around 04:00 UT and made it unstable. The unstable F2 started to erupt towards west-south direction. Lateron some part of this filament falls down to the solar surface and this material made unstable the filament F3. 

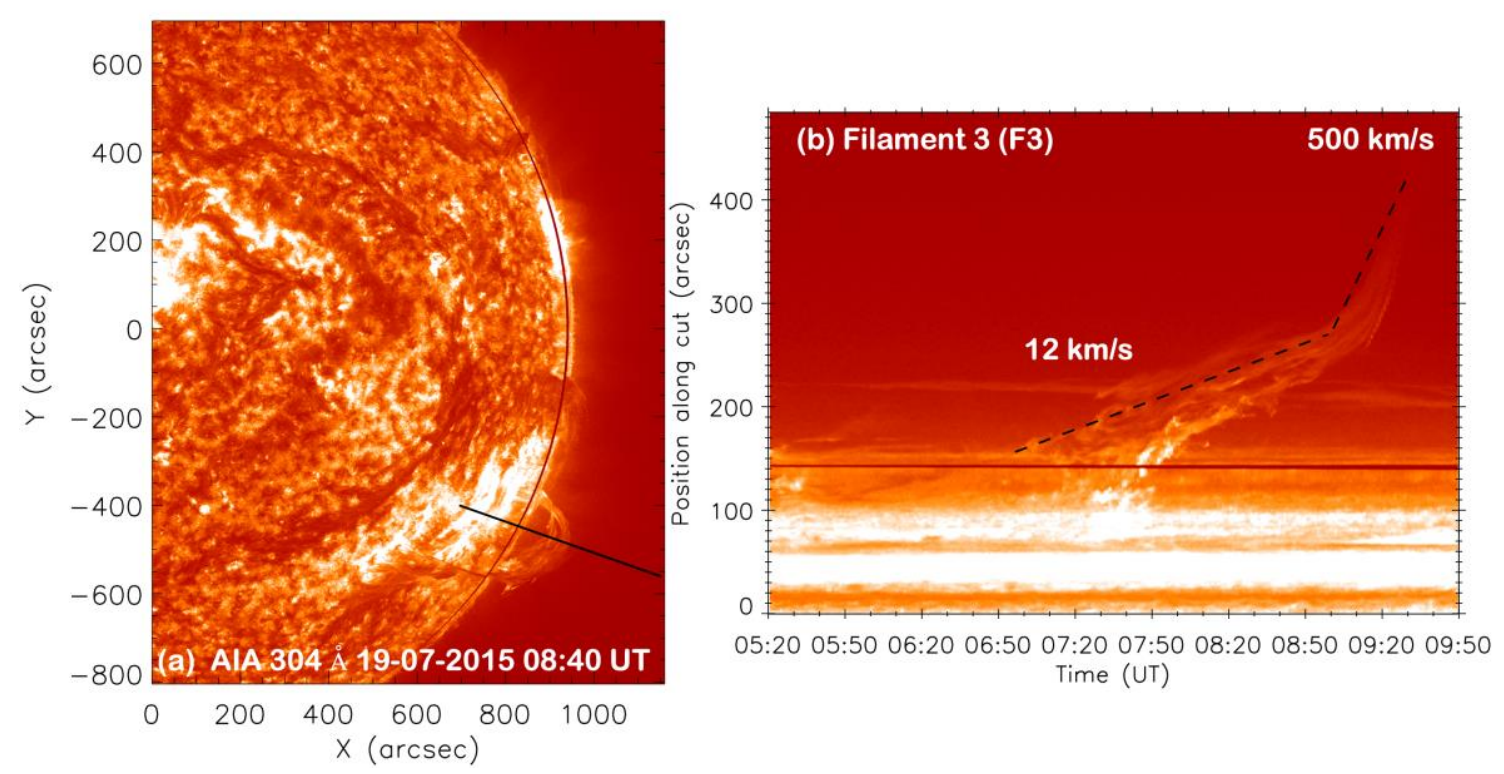

Figure 3: (a) Location of time slice selected for the F3 eruption direction (b) Corresponding timedistance plot.

Again, for the kinematics of filament F3 eruption, we have created a slice drawn in the left panel of Figure 3. The corresponding time-distance plot is presented in right panel of the same figure. From the time-distance plot, we have computed the eruption speed of F3 filament, which very clearly showed two eruptive phases (slow and fast). The speed of the slow phase is about $12 \mathrm{~km} / \mathrm{s}$, whereas the fast phase eruption speed is very fast and its average value is about 500 $\mathrm{km} / \mathrm{s}$. The two eruption phases are very clear and easily distinguishable. The slow rise phase is longer (about two hour), while the fast eruptive phase lasts about one hour.

\section{Summary}

We have studied three linked quiet filament eruptions that occurred on 2015 July 19, namely F1, F2 and F3. Initially the filament F1 starts to erupt due to some kind of instability, may be torus instability, and triggers the filament F2 eruption. The erupting F2 filament in turn causes the eruption of the filament F3. Finally F3 is fully erupted. The eruption of F3 filament was associated the a C2.1 solar flare and with a CME. This is a nice example of sympathetic eruption and can be explained by the MHD numerical simulation proposed by Török et al,. (2011). In future we plan to explore these sympathetic eruptions in more detail. 


\section{References}

Chandra, R., Filippov, B., Joshi, R., \& Schmieder, B. 2017, SoPh, 292, p. 81

Ding, J. Y.; Hu, Y. Q.; Wang, J. X.: 2006, SoPh, 235, p. 223

Forbes, T. G.: 2000, JGR, 105, Issue A10, p. 23153

Gilbert, H. R., Alexander, D., \& Liu, R. : 2007, Sol. Phys., 245, p. 287

Gopalswamy, N., Yashiro, S., Michalek, G., Stenborg, G., Vourlidas, A., Freeland, S., Howard, R., 2009. The SOHO/LASCO CME Catalog. Earth Moon and Planets 104, 295-313

Harvey, J. W., Bolding, J., Clark, R., et al. 2011, American Astronomical Society, SPD meeting, 42, p. 17.45

Jiang, Y., Shen, Y., Yi, B., Yang, J., \& Wang, J. 2008, ApJ, 677, 699

Joshi, Navin Chandra; Schmieder, Brigitte; Magara, Tetsuya; Guo, Yang; Aulanier, Guillaume: 2016; ApJ, 820, p. 126

Lemen, J. R., Title, A. M., Akin, D. J., et al.: 2012, Sol. Phys., 275, p.17

Lin, J., Soon, W., Baliunas, S. L.: 2003, New Astronomy Reviews, 47, Issue 2, p. 53

Moon, Y.-J., Choe, G. S., Park, Y. D., et al. 2002, ApJ, 574, p. 434

Munro, R. H., Gosling, J. T., Hildner, E., et al. 1979, Sol. Phys., 61, p. 201

Pesnell, W. D., Thompson, B. J., \& Chamberlin, P. C. 2012, SoPh, 275, p. 3

Priest, E. R., Forbes, T. G.: 2002, The Astronomy and Astrophysics Review, 10, Issue 4, p. 313

Tandberg-Hanssen, E., ed. 1995, Astrophysics and Space Science Library, 199, The nature of solar prominences Török, T.; Panasenco, O.; Titov, V. S.; Mikić, Z.; Reeves, K. K.; Velli, M.; Linker, J. A.; De Toma, G.: 2011; ApJL, 739, L63

Wang, Rui; Liu, Ying D.; Zimovets, Ivan; Hu, Huidong; Dai, Xinghua; Yang, Zhongwei: 2016; ApJL, 827, L12

Wang, Y., Sheeley, N. R., Jr., \& Rich, N. B. 2007, ApJ, 658, 1340

Yang, J., Jiang, Y., Zheng, R., Bi, Y., Hong, J., Yang, B.: 2012; ApJ, 745, p. 9

\section{Acknowledgments}

The AIA data used here is the courtesy of SDO (NASA) and AIA consortium. This work also utilizes data obtained by the Global Oscillation Network Group (GONG) Program, managed by the National Solar Observatory, which is operated by AURA, Inc. under a cooperative agreement with the National Science Foundation. The work is supported by the Bulgarian Science Fund under Indo-Bulgarian bilateral project INT/BLG/P-11/2019 (KP-06India/14 19 -Dec-2019). 


\title{
New 30-cm Solar Telescope at National Astronomical Observatory Rozhen
}

\author{
Tsvetkov Ts., Petrov N. \\ Institute of Astronomy and National Astronomical Observatory, BAS, Bulgaria \\ E-mail: tstsvetkov@astro.bas.bg
}

\begin{abstract}
A new solar telescope is about to be established at National Astronomical Observatory Rozhen, Bulgaria. Its construction and commissioning mark a new chapter in the development of solar observations in the region after the beginning of coronal monitoring in 2005. The new $30-\mathrm{cm}$ reflector is going to be focused on chromospheric observations. Its main parameters, scientific purposes and development status are presented in the current work.
\end{abstract}

\section{Introduction}

Over the last two decades there is a serious progress regarding new possibilities and methods of solar observations and their physical interpretation. Nevertheless, the modern solar physics is still facing lots of unanswered questions. Finding the answers is directly bound to a permanent monitoring of different manifestations of solar activity. There are ground-based and space-based observations now. The space-based observatories (e. g. SOHO, STEREO, SDO, etc.) together with the modern ground-based solar telescopes make possible to take a look at the Sun in a large range of the electromagnetic spectrum - gamma rays, ultraviolet, visible and infrared light, radio waves. This allows studies of the same active processes at different layers of solar atmosphere (so called "solar tomography"). All these observations widen our understandings of the physics of the Sun.

Historically, the development of Bulgarian astronomy naturally leads to the development of solar research. We owe its historical roots to the activity of Prof. Marin Bachevarov - Dean of the Faculty of Physics and Mathematics of Sofia University, Rector of the University and a pioneer for the construction of the first modern observational astronomical instrument in Bulgaria - a 6-inch refractor. From 1899 to 1905 Prof. Bachevarov, together with a group of students, began regular observations of sunspots from the Observatory in Sofia [Petrov N., et $a l ., 2018]$. Years later, in 1961, the first research expedition to observe a total solar eclipse on February 15 was organized in Bulgaria [Dermendjiev V. N., et al., 1999].

In the very beginning of 1990s the foundations of modern observational solar physics were laid in Bulgaria by Prof. Vladimir Dermendzhiev, who built solar tower with $15-\mathrm{cm}$ refractor for white-light observations of active processes. It was the first important step to the realization of the idea of own observational base in the Institute of Astronomy. As a result of even more efforts, a solar coronagraph was built in the solar tower of National Astronomical Observatory (NAO) Rozhen in 2005 [Petrov N., et al., 2018]. The renovation and progress of solar observational infrastructure in Bulgaria continued in 2019 with laying the foundations of building the first chromospheric telescope in Bulgaria.

This paper gives a preliminary information about the project for construction of new solar telescope in Bulgaria - its future location, main characteristics, scientific purposes and development status.

\section{Scientific purposes}

Scientific purposes change over time. New objectives appear due to the development of solar physics. The telescope should, therefore, be designed so as to be adapted to new scientific challenges. Bellow, we present objectives of the new 30-cm solar telescope planned to installed in NAO Rozhen, which can be formulated in terms of requirements of modern solar and solarterrestrial physics. 
Nowadays ground-based imaging of the Sun can be used as a complement monitoring of the solar activity. Its main advantages in the era of space-borne instruments are the relatively low cost of implementation and the ability access the received data immediately.

A $\mathrm{H} \alpha$ solar observations reveal the active nature of the solar atmosphere. The interaction between the convective motions in the photosphere and the dynamics in the hot corona happen in-between in the chromosphere - a layer where the kinetic energy of the plasma gradually becomes weaker than the energy of the magnetic field and the plasma parameter $\beta$ shifts from $\beta>1$ (photosphere) to $\beta \leq 1$ [Rodriguez Gomez J. M., et al., 2019] before reaching low-beta values in the corona and in the active region chromosphere $(\beta<<1)$ [Sakurai T., 2017]. The chromosphere is also a region from solar atmosphere where the temperature rises in height as it is situated right above the temperature minimum layer.

Prominences/filaments, fibrils, Ellerman bombs, flares and more are only part of the phenomena observable in $\mathrm{H} \alpha$. One of the main scientific purposes of the new solar telescope is dedicated to make regular observations of various manifestations of solar activity. A free-access online database is going to be established and updated as soon as the daily observations begin.

A network with amplified magnetic field situated above the photospheric network is visible in the chromosphere during $\mathrm{H} \alpha$ observations. It is called chromospheric network and is outlining the supergranulation cells. A downward flow of material with typical velocity of about 1-2 $\mathrm{km} \mathrm{s}^{-1}$ marks their boundaries [Rieutord M. and Rincon F., 2010]. Bright streams of luminous gas about $10000 \mathrm{~km}$ high, called spicules originate from the chromospheric network [Sterling A. C., 2000]. They are one of the smallest Quiet-Sun structures available for observations. The surface of the spicules is assumed as the actual boundary between the chromospheric and coronal material.

\section{Telescope parameters and capabilities}

The telescope is currently under construction. It is designed as Schmidt-Cassegrain reflector with diameter of the main mirror $\mathrm{D}=305 \mathrm{~mm}$ and a focal length $\mathrm{F}=3050 \mathrm{~mm}$ (Figure 1). It is equipped with Hinode solar guider and a flat $\mathrm{K} 8$ glass filter with a bandwidth $6560 \pm 500$ $\AA$ that provides reflection of at least $94 \%$ of the light in non-working areas of the spectrum to prevent overheating. A system of telecentric lens and a focal reducer $(0.75 \mathrm{x}-0.4 \mathrm{x})$ provides the opportunity for correction of the focal length. The effective focal length $F_{\text {eff }}$ will vary in range 5000 to $15000 \mathrm{~mm}$ and, respectively, the field of view will between $2.5 \times 2.5$ arcmin and $10 \times 10$ arcmin. With this telescope, we will be able to obtain observational material from active regions in the solar chromosphere with a line-of-sight velocity resolution between 0 and $10 \mathrm{~km}$ $\mathrm{s}^{-1}$. At the end of the tube will be placed a $\mathrm{H} \alpha$ filter $(\lambda=6562.8 \AA)$ with a bandwidth $\sim 0.3 \AA$ and a possibility for displacement of the centre of the bandpass at $\pm 0.5 \AA$ by $0.1 \AA$ step. The telescope was planned to receive its first light from the territory of NAO Rozhen in the first half of 2020, but now the deadline is indefinitely extended due to the pandemic situation. 


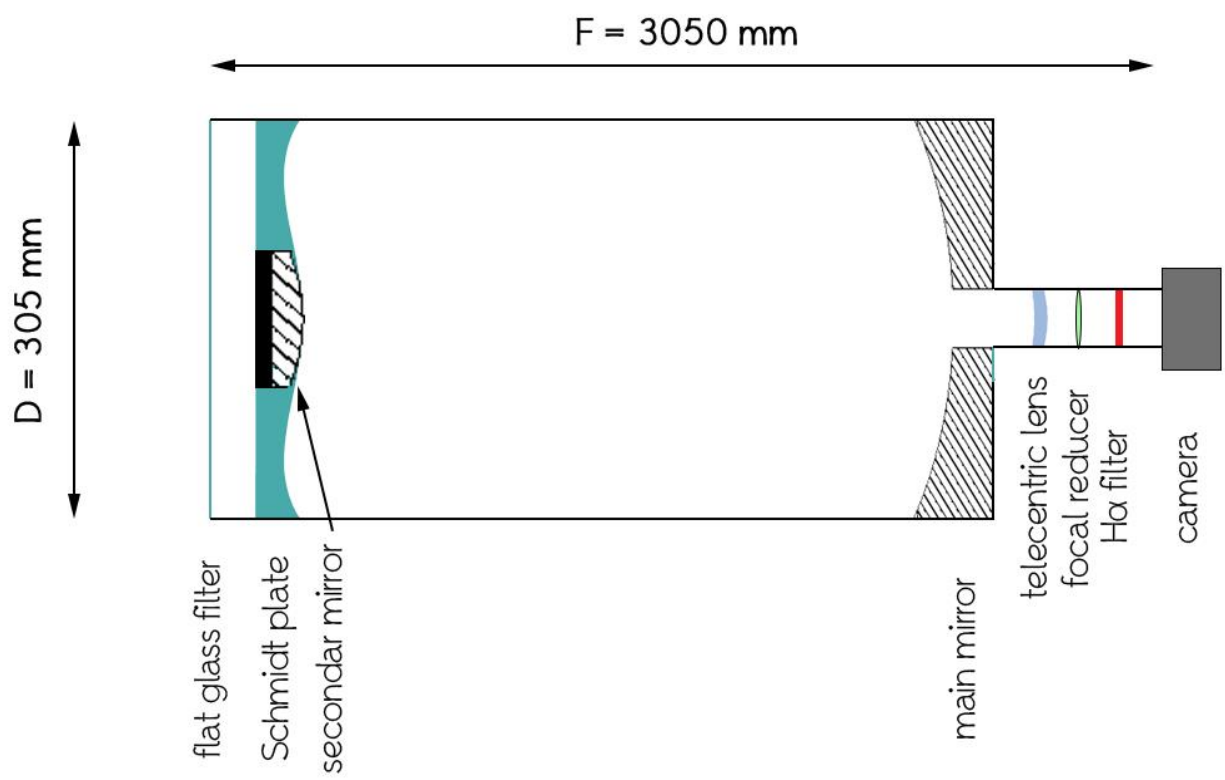

Fig. 1 Optical scheme of the Schmidt-Cassegrain telescope.

Nevertheless, the optical part of the telescope is already constructed and the first calibration test image, taken at the factory is now released (Figure 2). It is captured when to the telescope $\mathrm{F} / 10 \mathrm{~F}=3050 \mathrm{~mm}$ is attached a telecentric focal extender $4.3 \mathrm{x}$ equal to $\mathrm{F} / 43 \mathrm{~F}=13115 \mathrm{~mm}$ and a focal reducer $0.7 \mathrm{x}$ that, finally, makes $\mathrm{F}=9180 \mathrm{~mm}$. The scale of the image is $0.13 \mathrm{H} / \mathrm{px}$ in unfavourable weather conditions with high seeing.

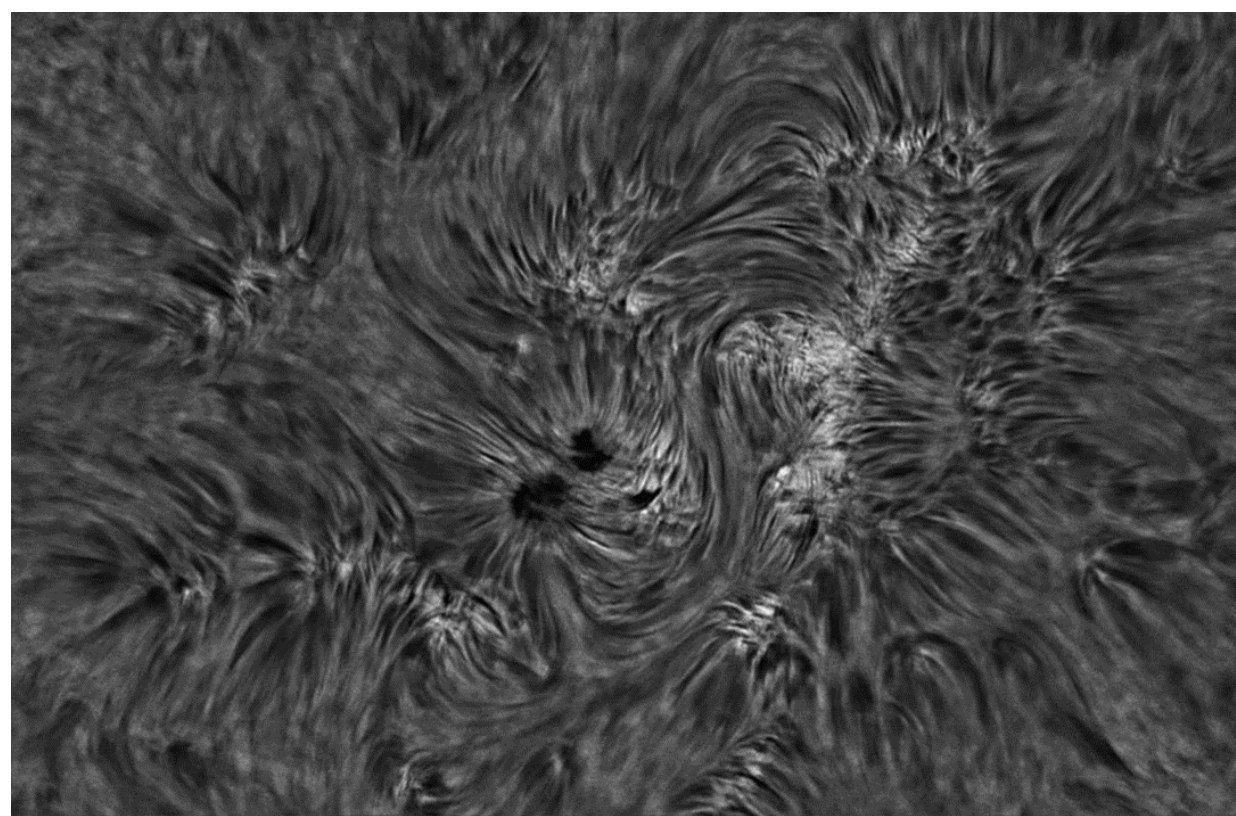

Fig. 2 A preliminary shot of the telescope, taken on 2020 August 10, showing the active region 12770.

The telescope will be equipped with a digital camera with high-performance, high-quality imaging by combining FLIR expertise with the latest in CCD and CMOS technology.

Apart from the operational software for telescope control, for the analysis of the observational data, a new software product founded on existing procedures in the IDL-based software product SolarSoftware will be developed. Precise data processing is the basis for quality scientific production, which necessitates the development of a specific algorithm that 
will facilitate and universalize the preliminary data analysis. Processed data will be provided through a specially designed website.

Upon its completion, the telescope will serve same tasks, both in regular solar observations and in the effective training, qualification and educational activities related to heliophysics and astronomy.

\section{Conclusions}

We present an overview of the capabilities and technical specifications of the new $30-\mathrm{cm}$ solar telescope that is going to receive its first light from the territory of NAO Rozhen soon. The telescope suggests a $\mathrm{H} \alpha$ view to the structures and dynamics of solar chromosphere in precise detail. Unlike most of the $\mathrm{H} \alpha$ telescopes used today it is not going to capture full disk images of the Sun. The changeable field of view that varies between $2.5 \times 2.5 \operatorname{arcmin}$ and $10 \times 10$ arcmin will offer observations of particular separate chromospheric parts. A database with free access that is going to archive daily observations is currently under construction.

\section{Acknowledgment}

This study is supported by the National Science Fund of Bulgaria with contract No. KP-06M38/3.

\section{References}

Dermendjiev, V. N., Mishev, D., Koleva, K. (1999). Solar eclipses. Prof. Marin Drinov Academic Publishing House, Sofia, Bulgaria.

Petrov, N., Kjurkchieva, D., Tsvetkov, Ts. (2018). Astronomical and Astrophysical Transactions, Vol. 30, Issue 4, pp. 441-452.

Rieutord, M., Rincon, F. (2010). Living Reviews in Solar Physics, vol. 7, article number: 2.

Rodriguez Gomez, J. M., Palacios, J., Vieira, L. E. A., Dal Lago, A. (2019). The Astrophysical Journal, Vol. 884, Issue 1 , article id. 88, 7 pp.

Sakurai, T. (2017). Proceedings of the Japan Academy, Series B Physical and Biological Sciences, vol. 93(2), pp. 87-97.

Sterling, A. C. (2000). Solar Physics, vol. 196, p. 79.

Yashiro, S., Gopalswamy, N., Michalek, G., St Cyr, O.C., Plunkett, S.P., Rich, N.B. and Howard, R.A. (2004). J. Geophys. Res., 109, 7105. 


\title{
Bulgarian Expedition for the Total Solar Eclipse on 2020 December 14
}

\author{
Tsvetkov Ts. ${ }^{1}$, Petrov N. ${ }^{1}$, Miteva R. ${ }^{2}$, Ivanov E. ${ }^{3}$, Popov $V .{ }^{3}$ \\ ${ }^{1}$ Institute of Astronomy and National Astronomical Observatory, BAS, Bulgaria \\ ${ }^{2}$ Space Research and Technology Institute, BAS, Bulgaria \\ ${ }^{3}$ Konstantin Preslavsky University of Shumen, Bulgaria \\ E-mail: tstsvetkov@astro.bas.bg
}

\begin{abstract}
Total solar eclipses are not only an impressive phenomenon, but also a chance for scientists to find the answers of some still unanswered questions. Even today, in the era of space-based solar observations, total solar eclipses provide opportunities for studying the physical processes of the corona and the solar-terrestrial interactions. The possibility of studying the outermost layer of the solar atmosphere in details is important part of astronomy since the models of its formation and dynamics are the basis for understanding the structure of all other sun-like stars in our galaxy.

We are preparing an expedition to observe the total solar eclipse on 2020 December 14 from Argentina and here we present our preliminary research of choosing the location, preparing the equipment and planning the experiments.
\end{abstract}

\section{Introduction}

The total solar eclipses (TSEs) reveal some already established global parameters of the solar corona such as its shape, electron density, physical parameters of temperature and the magnetic field, as well as the presence of emission lines of highly ionized chemical elements. And yet the contemporary observation methods show us that the physics of dynamic processes related to energy transport in the corona, the connection between local active processes in the lower solar atmosphere and coronal mass ejection are not yet well studied. Every TSE is unique itself in its details and in actual fact an exact repetition could never be observed. To expect local active processes on the Sun and in the solar corona, the phase of solar activity (mainly connected to the 11-year cycle of solar activity) is of great importance. The presence of those activity processes (sunspots and prominences mostly) provides the basis for the solar-terrestrial interactions and determination of the space weather.

A total solar eclipse will occur on 2020 December 14 for observers situated in South America. The Moon's umbral shadow will successively pass through the South Pacific Ocean, central parts of Chile and Argentina and the South Atlantic Ocean [Mobberley, M., 2007]. A partial eclipse will be visible from a much wider corridor, which includes a few islands located west of South America, parts of Uruguay, Paraguay, Brazil, Bolivia, Peru, Ecuador as well as the southwestern parts of Africa (Angola, Namibia, Botswana, South Africa, and others.).

The greatest eclipse will be visible from central Argentina, near Sierra Colorada (about $1000 \mathrm{~km}$ southwest of Buenos Aires $)-40^{\circ} 20.135^{\prime} \mathrm{S}$ and $67^{\circ} 57.533^{\prime} \mathrm{W}$, where the totality $\begin{array}{llll}\text { will continue } & 2 & \mathrm{~min} & 09.8\end{array}$

(http://xjubier.free.fr/site_images/TSE_2020/TSE_2020_GeneralCircumstances.jpg).

We already did a preliminary research for choosing the best location on the path of totality for scientific observations. Taking into account the weather conditions we decided to position our team at Rio Negro province (Figure 1a). Data about the average cloud amount in December from MODIS instrument onboard Aqua satellite helped us choose a spot about $90 \mathrm{~km}$ northwest from Los Menucos (Figure 1b). Eclipse details for the preferred location (BG on Figure 1b) compared to these for the point of greatest eclipse (GE on Figure 1b) are listed in Table 1. 
Table 1. Eclipse details for the chosen observational area near Los Menucos (BG) compared to the spot of greatest eclipse (GE) (https://www.timeanddate.com/eclipse/solar/2019-july-2).

\begin{tabular}{|c|c|c|c|c|c|}
\hline Location & Magnitude & $\begin{array}{c}\text { Duration } \\
\text { (C1 to C4) } \\
\text { [hh:mm:ss] }\end{array}$ & $\begin{array}{c}\text { Duration of } \\
\text { the totality } \\
\text { [mm:ss] }\end{array}$ & $\begin{array}{c}\text { Maximum } \\
\text { [UT] }\end{array}$ & $\begin{array}{l}\text { Sun's altitude } \\
\text { at maximum }\end{array}$ \\
\hline BG & 1.01262 & $2: 50: 36$ & $2: 09.7$ & $16: 12: 17$ & $72.7^{\circ}$ \\
\hline GE & 1.01268 & $2: 51: 38$ & $2: 09.8$ & $16: 13: 30$ & $72.7^{\circ}$ \\
\hline
\end{tabular}

Another important aspect of the chosen location is its altitude $-980 \mathrm{~m}$ above the sea level, which contributes to greater transparency of the atmosphere. The observational are is secluded place, situated outside big cities in uninhabited part of Argentina to reduce effects of light pollution or unforeseen interruptions by random citizens.

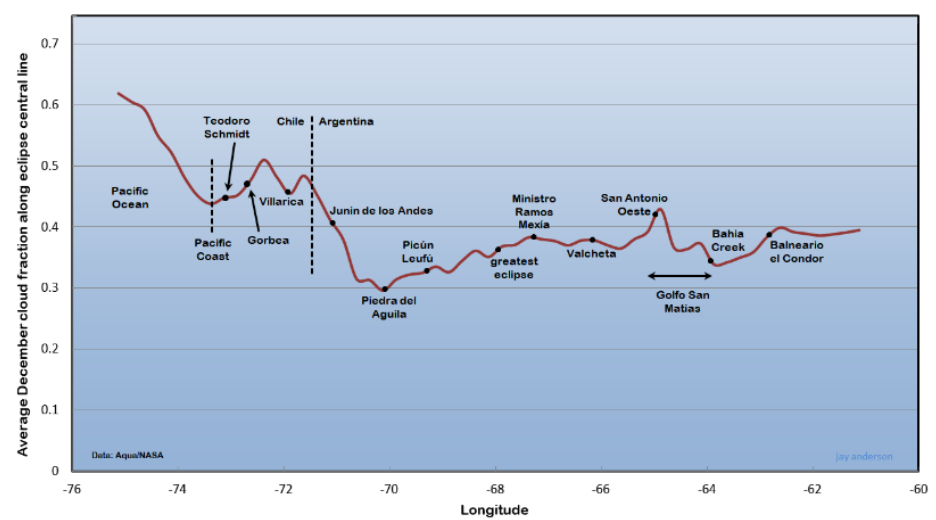

a)

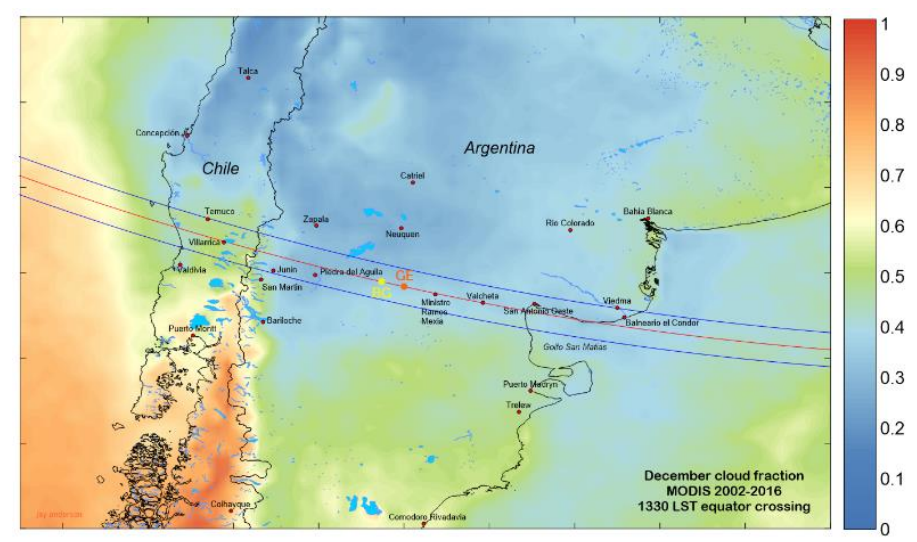

b)

Fig. 1 a) Average cloudiness along the centerline of the eclipse in December. $b$ ) Average December fractional cloud amount along the eclipse track over South America. BG notes the location that we chose and GE - the point of greatest eclipse. Data are extracted from 17 years (2002-2015) of observations from the Aqua satellite. (https://eclipsophile.com/tse2020/).

\section{Planned experiments and tasks}

\section{a) White-light observations of solar corona}

It is well known that the shape of the corona is subject to significant variations during the solar cycle [Gulyaev R. A., 1997]. The quantitatively description of the general coronal shape can be estimated by the method first suggested by Ludendorff more than 90 years ago by the definition of the flattening index [Ludendorff H., 1928]. For the purpose we need to obtain long exposure photos of the white-light corona. On the other hand, processing various pictures of the corona with different lenses, exposure times and cameras can reveal the fine structure of the corona.

We prepared a couple of different instruments to photograph the corona with different lenses, exposure times and cameras to be able to capture the shape of the white-light corona at high altitudes, but also to be able to combine different images to achieve details of coronal fine structure [Pasachoff J. M., et al., 2007]. Structures hard to be seen before a few decades, such as tiny loops, inhomogeneities in coronal streamers and cavities, polar plumes, etc. are now becoming observable. Such data, in combination with the ones from space-based solar observatories, are also useful for investigation of the connections between the different processes and formations in the solar atmosphere.

\section{b) Polarimetric observations of corona and prominences}


TSEs offer an opportunity to measure the polarization of the coronal emission at altitudes up to 5 solar radii. Since the intensity of the white-light corona is in linear correlation with the density of the free electrons, the polarimetric observations allow not only differentiation of the emission from different components of the corona ( $\mathrm{K}$ - and $\mathrm{F}$ - corona), but also a direct measurements of coronal density.

Our task will include determination of the degree of polarization in the different parts of the corona. We also plan to investigate the electron distribution in the corona as a function of the distance from the centre of the Sun and the relationship between the degree of polarization and the wavelength. A comparison with white-light data will reveal the changes in the regions around the coronal structures.

In case that there are prominences visible on the solar limb during the eclipse we will pay special attention on their surroundings and supporting magnetic structures. The polarimetric observations will also be useful for determination of solar magnetic field parameters in these regions [Lin H., 2005].

\section{c) Shadows bands and atmospheric conditions during the eclipse}

In the last moments right before and after the totality an atmospheric phenomenon called "shadow bands" that occurs as a result of interference of the light emitted from a thin solar crescent being refracted by the Earth's turbulent atmosphere could be observed [Codona J. L., 1998]. Although they were first noticed almost three centuries ago [Littmann M., et al., 2009], but even nowadays they are not fully studied yet, because of the difficulties connected to their registration.

During our expeditions for total solar eclipse on 2019 July 2 we performed a relatively new experiment looking for the possible influence on the shadow bands pattern by the wind [Tsvetkov Ts., et al., 2019]. We successfully performed a videotaping of the bands and measured the wind speed and direction (4 times/second) using ultrasound anemometer. It is largely accepted that the "eclipse winds" depend on the topography of the terrain. Since our observational area was situated in the outskirts of the Atacama Desert and only $35 \mathrm{~km}$ east of the Pacific Ocean, we plan to reproduce the experiment again during the TSE 2020 as we plan to be located at the inner part of South America (Figure 1b). A possible successful implementation of the experiment will assure the possibility to compare the results and form hypothesis on the relationship between the wind properties and the shadow bands observed during TSEs.

Another aspect of the planned experimental tasks is connected to the eclipse meteorology. In addition to the wind properties, we will monitor the air temperature near the ground during the different phases of the eclipse as it usually drops between $5^{\circ}$ and $15^{\circ}$ during the totality [Calmas D. M., et al., 2019; Szalowski K., 2002].

\section{Conclusions}

For almost a year and a half South America will host the umbral path for a second time on 2020 December 14. Our team plans an expedition to observe the totals solar eclipse from the territory of Argentina. We already chose an observational location in Patagonia region, at Rio Negro province far from in inhabited places at almost $1000 \mathrm{~m}$ altitude.

The tasks we plan to perform are dedicated to 3 main topics: observations of the whitelight solar corona, registration of polarized emission from the corona and typical coronal structures, detection of the pre- and post-totality shadow bands and monitoring the meteorological parameters of the wind and air temperature during the eclipse.

Some of the planned experiments are similar to the obtained during 2017 August 21 and 2019 July 2 total solar eclipses observed in the USA and Chile, respectively, and the results will be compared after the upcoming expedition. 


\section{Acknowledgment}

This study is supported by the National Science Fund of Bulgaria with contract No.: KP06-H28/4 and the Bulgarian Ministry of Education and Science under the National Research Programme "Young scientists and postdoctoral students" approved by DCM \#577/17.08.2018.

\section{References}

Calamas, D. M., Nutter, C., Guajardo, D. N. (2019). Int J Energy Environ Eng 10, 147-156.

Codona J. L. (1998). Astronomy and Astrophysics, 164, no. 2, 415-427.

Gulyaev, R. A. (1997). Astronomical and Astrophysical Transactions, vol. 13, Issue 2, pp.137-144.

Lin, H. (2005). Astronomical Polarimetry: Current Status and Future Directions, vol. 343, 357.

Littmann, M., Espenak, F., Willcox, K. (2009). Totality: Eclipses of the Sun, Oxford University Press.

Ludendorff, H. (1928). Sittungsberichte Preuss. Akad. Wiss., Phys.-Math. IClasse 16, 185.

Mobberley, M. (2007). Total Solar Eclipses and How to Observe Them, Springer, New York, US.

Pasachoff, J., Rušin, V., Druckmüller, M., Saniga, M. (2007) The Astrophysical Journal, 665, Issue 1, 824-829.

Szalowski, K. (2002). Journal of Atmospheric and Solar-Terrestrial Physics, 64, 1589-1600.

Tsvetkov, Ts., Miteva, R., Ivanov, E., Popov, V., Nakeva, Y., Bojevski, L., Damm, T., Petrov, N. (2019).

Proceeding of Fifteenth International Scientific conference "Space, Ecology, Safety - SES2019", held 6-8 November 2018 in Sofia, Bulgaria, 52-56. 


\title{
Functional Dependence of the Critical Frequency of the Ionospheric F2-layer on Geomagnetic Activity
}

\author{
Bojilova R. ${ }^{1}$, Mukhtarov P. ${ }^{1}$ \\ ${ }^{1}$ National Institute of Geophysics, Geodesy and Geography, Bulgarian Academy of Sciences, \\ Acad. G. Bonchev St, Bl. 3, 1113 Sofia, Bulgaria \\ E-mail: rbojilova@geophys.bas.bg
}

\begin{abstract}
.
This paper presents the functional dependence of the ionospheric characteristic foF2, representing the critical frequency of the ionospheric F2-layer, on the geomagnetic activity produced by regression analysis. The data for the ionosphere were obtained from the ionosonde station at Sofia (National Institute of Geophysics, Geodesy and Geography, Bulgarian Academy of Sciences) for the period of 1995-2014. The geomagnetic activity is represented by the planetary Kp-index derived from the NOAA. Some examples defining the use of both filtered Kp values and relative deviations of foF 2 are shown in this study. The results of the obtained seasonal dependencies demonstrate that the response in winter is weaker than that in summer and equinoxes. The considered dependencies are significantly nonlinear, requiring a thirddegree polynomial approximation. It is noted that the range of the geomagnetic activity variation is different in different months; this does not allow reliable values of the average response to strong geomagnetic disturbances to be obtained in some cases. The differences found by using the regression analysis between the ionosphere responses in day and night conditions necessitate the generation of an empirical foF 2 model separately for day and night conditions. These results can be used to establish an empirical model for predicting the state of the ionosphere over Bulgaria.
\end{abstract}

\section{Introduction}

The ionospheric structure and variability are related to changes in solar radiation and geomagnetic activity, together with the subsequent response of the thermosphere-ionosphere system [Roble, 1995]. The ionosphere also varies in response to neutral winds [Schunk et al., 2009], electrodynamical coupling with the overlying plasmasphere and magnetosphere [Huba et al., 2005], and dynamical coupling with the underlying atmosphere particularly effective during low solar activity conditions [Mendillo et al., 2002; Rishbeth, 2006]. Geomagnetic storms are generated through the interaction between coronal mass ejections and the Earth's magnetic field. Such disturbances can lead to a significant perturbation of the "quiet-time" ionosphere due to large variability in the electron density distribution, total electron content (TEC) and the ionospheric current system [Prölss, 1995].

The midlatitude F2-layer response to geomagnetic storms is mainly based on storm-induced changes in the neutral atmosphere, which are primarily a consequence of a strong Joule heating in the auroral thermosphere. At lower heights the role of the ionization and photochemical processes increases due to shorter electron lifetimes. The altitude dependence of the ionospheric response is due to the differences in physical mechanisms responsible for the changes of the electron concentration. While in the $\mathrm{E}$ and $\mathrm{D}$ regions the primary reason for the electron concentration changes is the variation of the ionization rate because of corpuscular intrusions, in the F2 layer the electron density variations are predominantly caused by such factors as neutral composition changes and dynamical processes. 
There is one more difference in the electron concentration behavior in various ionospheric regions. All "disturbances" in the lower ionosphere (poststorm effects, auroral absorption, polar cap absorption (PCA)) are actually an increase of electron concentration above some "quiettime" background level. Contrary to that, the F2-layer response to a geomagnetic disturbance (so called ionospheric storm) consists of effects of both signs. Both the depletion and enhancement of the electron density relative to the background level are observed during storms and are called "negative" and "positive" phases of the storm, respectively.

The latitude and longitude, season, as well as both the storm onset time and maximum are the main factors which define the positive/negative response [Mukhtarov et al., 2013; Mukhtarov and Bojilova, 2017].

The ionospheric storms are usually manifested by variations of the electron concentration in a wide altitude range from about $200 \mathrm{~km}$ to $800-1000 \mathrm{~km}$. Therefore, the basic feature of the storms is also the TEC variations observed from the satellite-to-ground radio wave propagation.

The basic aim of this study is to find a relationship between the critical frequency of the F2-layer and the geomagnetic activity represented by the Kp-index. The requested functional dependence between the considered parameters is obtained by applying regression analysis. The regressions of the seasonal dependencies are presented separately for day and night conditions and a proper justification for this is made. The results also confirm the expediency of introducing the relative deviation of the critical frequencies of the F2-layer from the geomagnetic activity as a third degree polynomial. All these results can be used for producing an empirical model for forecasting the state of the ionosphere over Bulgaria.

\section{Data and methods}

The geomagnetic activity, described by the planetary $\mathrm{Kp}$-index is provided from: https://omniweb.gsfc.nasa.gov/. The foF2 values are derived from the ionosonde station SofiaSQ143 $\left(42.4^{\circ} \mathrm{N}, 23.2^{\circ} \mathrm{E}\right)$ that belongs to the National Institute of Geophysics, Geodesy and Geography, Bulgarian Academy of Sciences.

To find the dependence of the ionospheric characteristics on the geomagnetic activity it is convenient to use not the specific values of these characteristics, which have a strong daily and seasonal course, but their relative deviations from the sliding medians. The relative deviations of the critical frequencies of the F2-layer are obtained using the formula:

$$
r f o F 2(t)=\frac{f o F 2(t)-f o F 2 m e d(U T)}{\text { foF } 2 \text { med }(U T)}
$$

From the value of the critical frequency of the F2-layer measured at some moment $t$ (noted by $f o F 2(t))$, subtract the median value measured at the same universal time for an interval of 15 days centered to the current day (marked as $f o F 2_{\text {med }}(U T)$ ) and the difference is divided by the same median value.

The filtered Kp (filt_Kp) is calculated by subtracting from each value in the time series the average one obtained for a segment of time with a length of 27 days and centered on the current time. This conversion ensures that the variations in the Kp-index with a time scale greater than 27 days are removed. This is very useful procedure because the relative values of the ionospheric characteristics do not contain variations with a time scale longer than 27 days.

A criterion used to determine whether an hour belongs to day- or night-time is the cosine of the Sun's zenith angle; the negative values indicate night-time conditions. The cosine values of the zenith angle $\chi$ are determined by using the simplified astronomical formula: 


$$
\begin{aligned}
& \cos \chi=\sin \left(\frac{\pi}{180} \varphi\right) \sin \varphi_{0}+\cos \left(\frac{\pi}{180} \varphi\right) \cos \varphi_{0} \cos \left(\frac{\pi}{180}(15(U T-12)-\theta)\right) \\
& \varphi_{0}=\frac{\pi}{180} 23.45 \sin \left(\frac{2 \pi}{365}(D O Y-21)\right)
\end{aligned}
$$

The formula includes the latitude and longitude of the station $\varphi$ and $\theta$ respectively, the universal time UT, and the serial number of the day of the year, counted from January 1, DOY.

\section{Results}

This section illustrates some examples of the group regressions between the relative values of the critical frequencies of the F2-layer and the filtered $\mathrm{Kp}$-index, presented in Fig. 1, for selected calendar months representing winter (January, upper row of plots), summer (July, middle row of plots) and equinoxes (September, bottom row of plots) as the regressions are given for day- (red color, left column of plots) and night-time (blue color, right column of plots) conditions.

A careful inspection of the regression dependencies shown in the Fig. 1 reveals a significant nonlinear relationship between the considered parameters, requiring a third-degree polynomial approximation. It is noted that the range of variation of geomagnetic activity is different in the different months, which does not allow in some cases (January) reliable values of the average response to strong geomagnetic disturbances to be obtained. This is due to the insufficient statistical material available for this study.

A characteristic feature of the anomalies of geomagnetic origin is the amplification of the deviations mainly in the negative direction, i.e. nonlinearity of the reaction is enhanced during strong magnetic storms, especially during the summer months. The comparison between the forms of the regressions separately for day- and night-time conditions indicates significant differences, especially during the winter months. For example, in January, the day-time dependence shows a positive (increasing) change with respect to the average values of the geomagnetic activity, whereas at night conditions, opposite, at high geomagnetic activity values, a negative change is observed. This can be explained by the predominance of the positive ionospheric response to geomagnetic storms in day-time winter conditions [Bojilova and Mukhtarov, 2019]. 

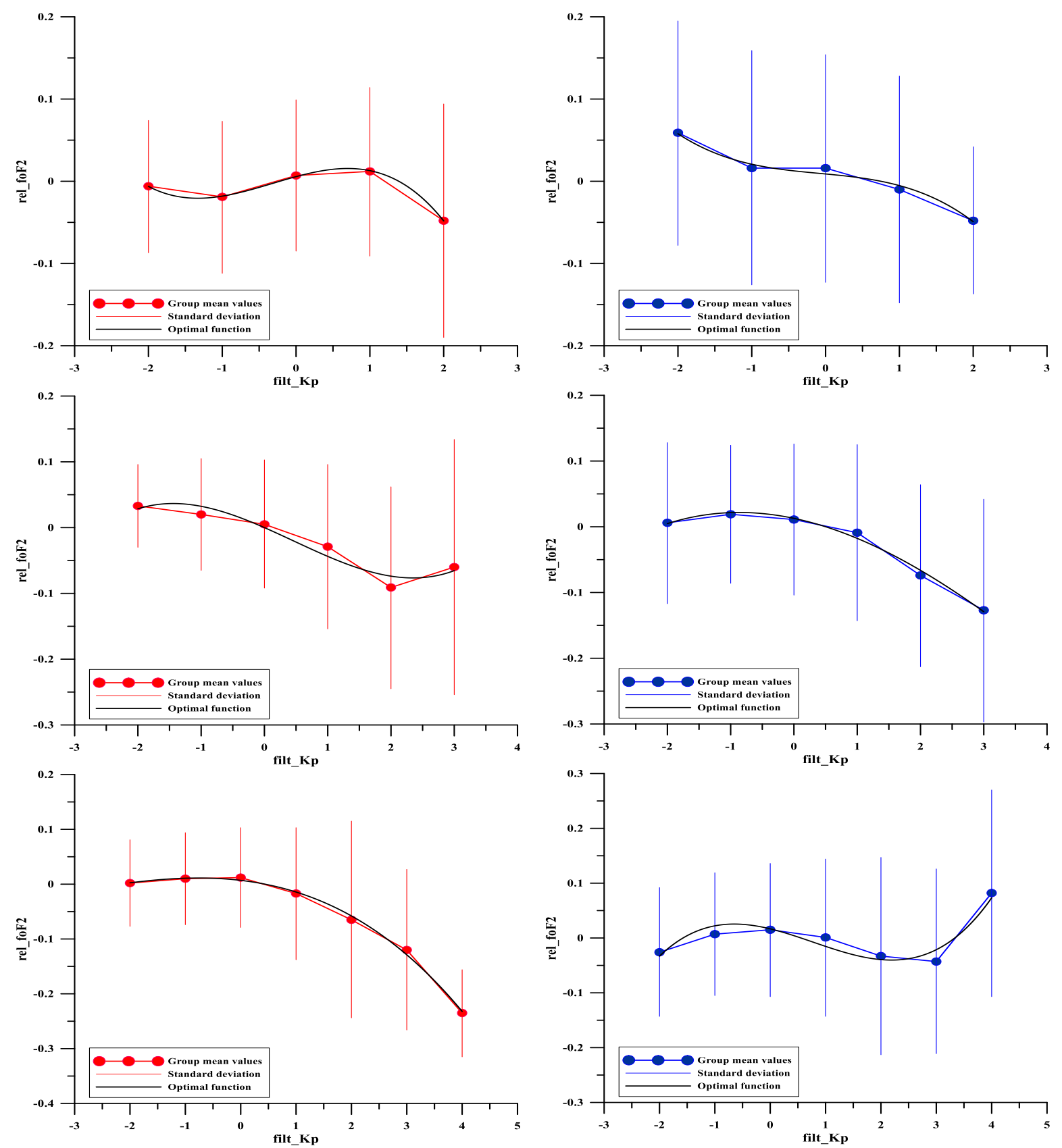

Fig. 1 Comparison of the monthly group regressions between the filtered values of the geomagnetic activity Kp-index and the relative values of foF 2 as the regressions are divided for day (left panel) and night (right panel), as well as their optimal approximations. The following months are shown: January (upper row of panels); July (middle row of panel) and September (bottom row of panel). The error bars are represented by the standard devisions.

There is no significant difference between day and night regressions in the summer and equinoctial months which have a definite negative course. This result reflects the known fact that the geomagnetic storms cause mainly negative reactions of the F2-layer critical frequencies in summer and equinoxes.

The results obtained will be used for producing of an empirical model intended to predict the F2-layer critical frequencies of the ionosphere over Bulgaria. 


\section{Conclusions}

The presented results in this study justify the use of both the relative values of the critical frequency of the F2-layer and the filtered the Kp-index. The necessity of introducing a third degree polynomial dependence of the relative deviation of the critical frequency of the F2-layer on the geomagnetic activity is also confirmed. The differences between the response of the F2layer critical frequency in the day and night conditions demonstrated in the presented regression analysis justify the necessity to synthesize an empirical model for forecasting the ionosphere separately for day and night. The results obtained will be used to establish an empirical model intended to predict the state of the ionosphere over Bulgaria.

\section{Acknowledgment}

This work was partially supported by the Bulgarian Ministry of Education and Science under the National Research Programme "Young scientists and postdoctoral students" approved by DCM № 577 / 17.08.2018 and by Project FA8655-12-1-57.

\section{References}

Bojilova, R., Mukhtarov, P. (2019). Response of the electron density profiles to geomagnetic disturbances in January 2005. Stud Geophys Geod 63, 436-454

G. Roble, R. (1995). Major greenhouse cooling (yes, cooling): The upper atmosphere response to increased CO2, Reviews of Geophysics, 33, 539-546.

Huba, J. D., Joyce, G., Sazykin, S., Wolf, R., \& Spiro, R. (2005). Simulation study of penetration electric field effects on the low-to mid-latitude ionosphere, Geophysical Research Letters, 32.

Mendillo, M., Rishbeth, H., Roble, R. G., \& Wroten, J. (2002). Modelling F2-layer seasonal trends and day-today variability driven by coupling with the lower atmosphere, J. Atmos. Sol.-Terr. Phys., 64(18), 1911-1931.

Mukhtarov, P., B. Andonov, and D. Pancheva (2013). Global empirical model of TEC response to geomagnetic activity, J. Geophys. Res. - Space Physics 118.10, 6666-6685.

Mukhtarov, P., R. Bojilova (2017). Influence of Solar and Geomagnetic Activity on the Ionosphere over Bulgaria, C.R. Acad. Bulg. Sci., 70 (9), 1289-1296.

Prölss, G.W. (1995). Ionospheric F-region storms. In: Volland H. (Ed.), Handbook for Atmospheric Electrodynamics, vol. 2, CRC Press, Boca Raton, pp. 195-248

Rishbeth, H. (2006). F-region links with the lower atmosphere? J. Atmos. Sol.-Terr. Phys, 68(3-5), 469-478.

Schunk, R., \& Nagy, A. (2009). Ionospheres: physics, plasma physics, and chemistry, Cambridge university press. 


\title{
Relationship Between Short-Term Variations in Solar Activity and Critical Frequency of the lonospheric F2 Layer
}

\author{
Bojilova R. ${ }^{1}$, Mukhtarov P. ${ }^{1}$ \\ ${ }^{1}$ National Institute of Geophysics, Geodesy and Geography, Bulgarian Academy of Sciences, \\ Acad. G. Bonchev St, Bl. 3, 1113 Sofia, Bulgaria \\ E-mail: rbojilova@geophys.bas.bg
}

\begin{abstract}
.
The present work is devoted to the finding of a functional dependence of the critical frequency of the ionospheric F2 layer, denoted by foF2, on the short-term variations in solar activity represented by the solar radio flux F10.7. The foF2 data are obtained from the ionosonde station at Sofia for the period of 1995-2014, while those for F10.7 were taken from the website of NASA https://omniweb.gsfc.nasa.gov/. The examples considered in this work demonstrate the need of pre-processing the selected parameters by introducing a relative deviation from the median value for each of the quantities. The use of a group regression to represent the required relationship is also justified. The result of the regression analysis revealed that the optimal functional dependence between the critical frequency of the F2 layer and F10.7 is close to linear one. These results can be used for building an empirical model that could predict the state of the ionosphere over Bulgaria.
\end{abstract}

\section{Introduction}

Solar radiation has a significant impact on the electron concentration of the ionosphere. The solar activity dependence of the ionosphere is fundamental issue in ionospheric physics, providing information essential for understanding the processes occurring in the ionosphere.

The ionosphere is an open system that strongly couples with the magnetosphere and thermosphere [Huba et al., 2005; Liu et al., 2011]. Various photo-chemical and chemical reactions and dynamical and electro dynamical processes in the system exchange and transport mass, momentum and energy in a complex manner. The regular and irregular variability of the solar activity strongly affects the chemical reactions and physical processes and it further strongly modulates the structure and development of the ionosphere and thermosphere. An essential issue in ionospheric physics is to study ionospheric characteristics and processes under different solar activity conditions. The study of solar activity modulation of the ionosphere will improve our understanding of the ionospheric structure and its development. This is critical in determining ionospheric climatology and long-term trends, and will also deepen our knowledge of chemical and physical processes in the ionosphere and thermosphere [Schunk and Nagy, 2009]. On the other hand, the solar activity dependence of the ionosphere is one of the core issues for ionospheric empirical models. Moreover, it is realized that the ionospheric climatology, especially the solar activity effects of the ionosphere, provides an insight into ionospheric weather problems.

Solar activity, as discussed in the scientific community, can have different meanings. First, it refers to the level of solar activity, indicating the intensity of solar electromagnetic ra-diation, particularly notable at wavelengths of solar X-rays and the extreme ultraviolet (EUV) (XUV). Second, refers to solar activity events; e.g. coronal mass ejection and solar proton events. The two meanings have a close relationship as well as distinct differences. Both the variability of solar activity and its relationship with the ionosphere have received a renewed interest recently. 
The Earth's upper atmosphere absorbs solar radiation, resulting in heating, dissociation and ionization. The ionosphere is mainly produced via the ionization effect of solar XUV. It is well established that the solar XUV fluctuates regularly and irregularly over timescales from minutes (flares) and roughly 27 days (solar rotation) to decades (11-year solar cycle), with amplitudes varying up to more than 1000 times. The variability of the solar activity initiates large variations in the neutral density and temperature, ion and electron densities and temperatures, neutral winds, and electric fields in the ionosphere [Rishbeth, 2006].

The purpose of this study is to find a relationship between the critical frequency of the F2layer and the solar activity, represented by F10.7. This work presents a regression analysis between the relative deviations from the median value of the considered parameters. The result of the analysis shows that the optimal function describing the relationship between the relative deviations of foF 2 and relative F10.7 is close to linear one. In the example shown, the hours at which the geomagnetic activity index $\mathrm{Kp}$ is greater than 5 are ignored. In this way the contradiction between variations in solar activity causing usually a positive ionospheric response and the geomagnetic disturbances that most often generate a negative response is significantly reduced. All these results can be used for establishing of an empirical model for forecasting the state of the ionosphere over Bulgaria.

\section{Data and methods}

Traditionally, in the ionospheric studies, the daily and seasonal fluctuations of the ionospheric characteristics and its dependence on long-term changes in solar activity are given by the monthly median of hourly values. The advantage of using the median value over the average one is due to the elimination of the extreme values connected with geomagnetic disturbances [Pancheva and Mukhtarov, 1996]. For this reason, the median value is used as a characteristic of the calm in the geomagnetic sense conditions of the ionosphere in a given calendar month. Traditionally, as a characteristic of solar activity, the smoothed values of the radiation flux of the Sun with a wavelength of $10.7 \mathrm{~cm}, \mathrm{~F} 10.7$, are used.

The values of the solar radio flux F10.7 are provided from: https://omniweb.gsfc.nasa.gov/. The foF 2 values are derived from the ionosonde station Sofia-SQ143 $\left(42.4^{\circ} \mathrm{N}, 23.2^{\circ} \mathrm{E}\right)$ that belongs to the National Institute of Geophysics, Geodesy and Geography, Bulgarian Academy of Sciences.

The use of raw F10.7 data is incorrect when using the relative values of the ionospheric characteristics in which the 11-year variability is filtered out. It is therefore necessary to work with the relative values of F10.7 obtained by the formula:

$$
r F 10.7(t)=\frac{F 10.7(t)-F 10.7_{m}}{F 10.7_{m}}
$$

where $\mathrm{F} 10.7 \mathrm{~m}$ is the average value calculated for a segment of time with a length of 27 days and centered on the current time. Thus the relative F10.7 values contain variations related to the rotational solar period and its harmonics but not variations due to the 11-year cycle of solar activity [Mukhtarov and Bojilova, 2017].

Fig. 1 shows a comparison of the raw values of solar radio flux F10.7 (marked in red) and the obtained relative values of the same parameter (blue) according to the formula above for October 2013. 


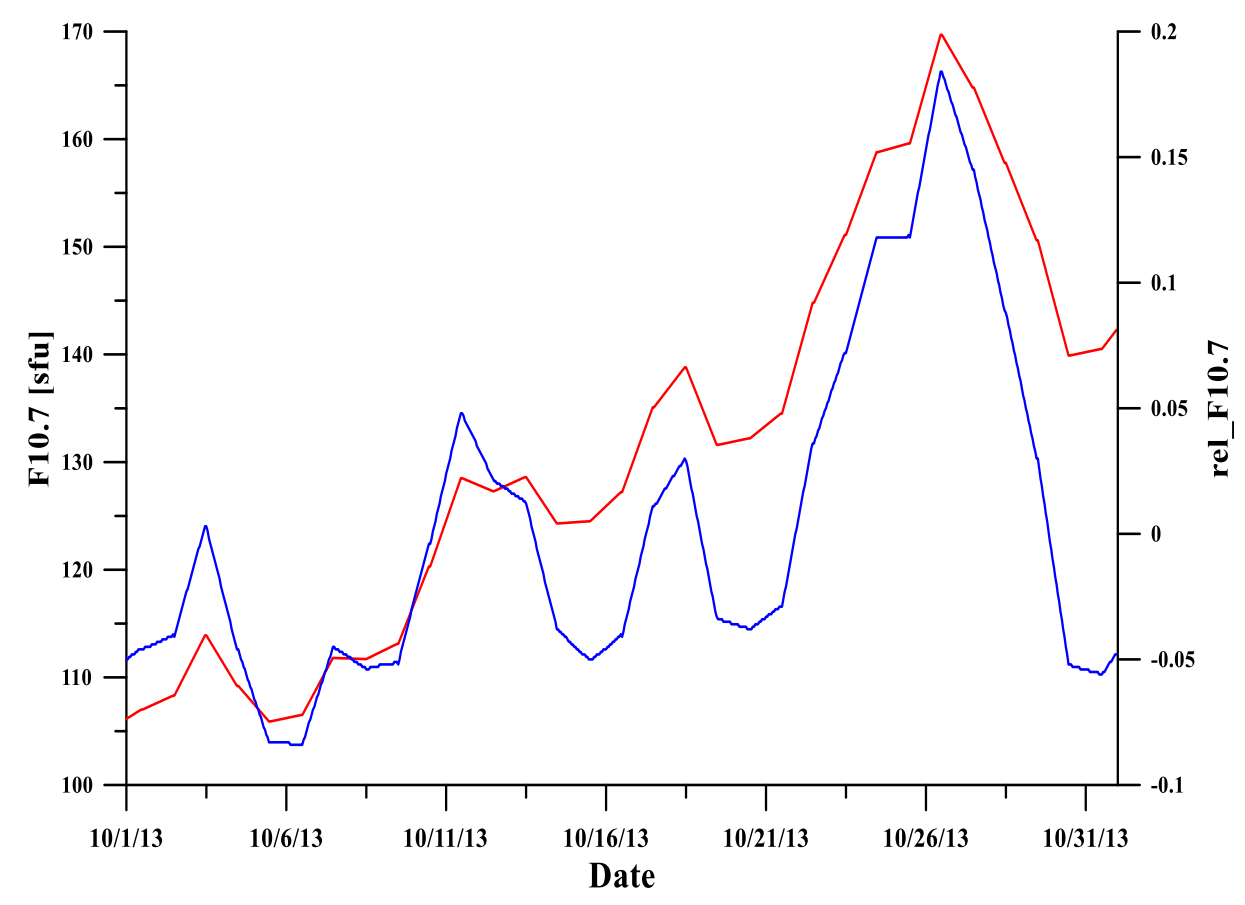

Fig. 1. Comparison between the values of F10.7 (red line) and the calculated relative values (blue line) for October 2013.

\section{Results}

To find an optimal functional dependence between the short-term variability of the solar activity and the ionospheric parameters foF2 we perform a regression analysis. The latter is between the relative values of F10.7 and the relative values of foF2. The obtained optimal functional dependence will be later the justification for producing an empirical model for forecasting the ionosphere over Bulgaria.

In this case the group regression analysis is used. For this purpose the range of variation of the „cause "value is divided into intervals, and for each such interval the mean values of the „cause" and the mean value of its corresponding "consequence" values are determined. The functional dependence is obtained by using the least squares method and is calculated on the basis of these group averages. The advantage of this approach in this case is based on the fact that solar and geomagnetic indices have many values under conditions close to calm ones (the modified indices under calm conditions have values close to zero) and only few values under disturbed conditions. If the regressions are calculated based on the regular manner, then the small cases representing the ionospheric response to strong solar variability will be suppressed by prevailing cases of mean solar activity.

Fig. 2 presents the group regression between the relative deviation of foF 2 and the relative values of solar activity, marked by rel_F10.7. The error in determining the average of rel_foF2 for a given rel_F10.7 interval is represented by the standard deviation. Fig. 2 also represents the number of values in a given interval of rel_F10.7 shown in red color. The graph clearly shows the predominance of cases of calm in solar activity. The number of cases with extreme elevation is two or more orders of magnitude smaller; this fact clearly confirms the correctness of using the group regression. The graph shows that the optimal function describing the obtained results is close to linear one; the latter provides justification for describing the ionospheric response to solar activity in the generating a model for the prediction of the ionospheric characteristics over Bulgaria. 


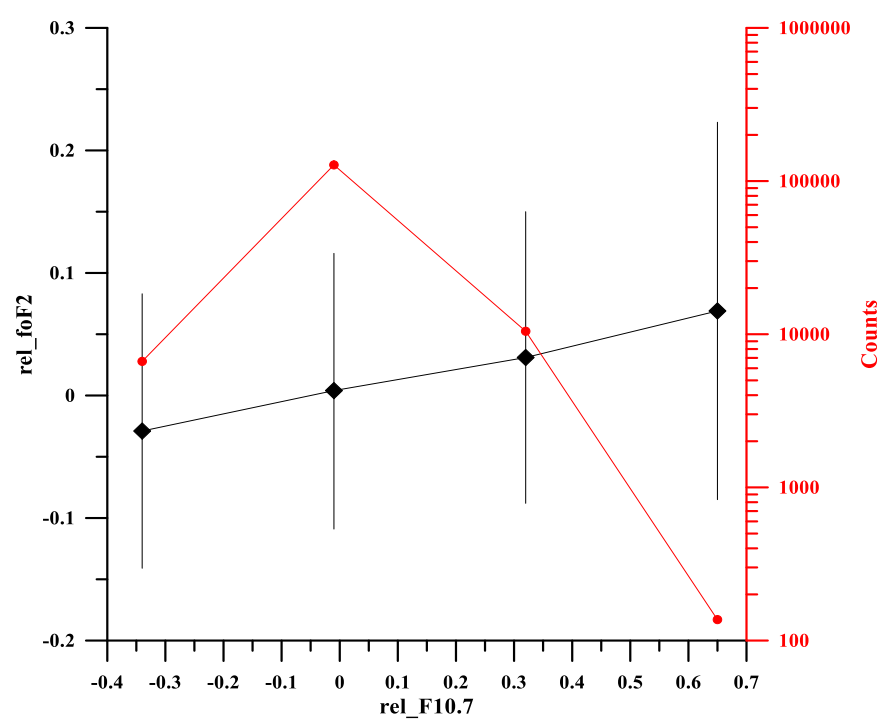

Fig. 2. Group regression between the rel_F10.7 and the relative deviations of the ionospheric characteristic foF 2 represented by black color line and the number of hits in the corresponding rel_F10.7 intervals shown in red color.

\section{Conclusions}

The group regression analysis revealed that the optimal relationship between the ionospheric critical frequency and the short-term variability of the solar activity over Bulgaria can be described by a linear function. It is important to note that this relationship is found only at times when the geomagnetic activity index $\mathrm{Kp}$ is below 5. In this way the contradiction between variations in solar activity that usually cause a positive ionospheric reaction and the geomagnetic disturbances that cause mostly negative response has been reduced. All these results can be used to produce an empirical model for predicting the state of the ionosphere over Bulgaria.

\section{Acknowledgment}

This work was partially supported by the Bulgarian Ministry of Education and Science under the National Research Programme "Young scientists and postdoctoral students" approved by DCM № 577 / 17.08.2018 and by Project FA8655-12-1-57.

\section{References}

Huba, J. D., Joyce, G., Sazykin, S., Wolf, R., \& Spiro, R. (2005). Simulation study of penetration electric field effects on the low-to mid-latitude ionosphere, Geophysical Research Letters, 32.

Liu, L., Wan, W., Chen, Y., \& Le, H. (2011). Solar activity effects of the ionosphere: A brief review. Chinese Science Bulletin, 56(12), 1202-1211.

Mukhtarov, P., R. Bojilova (2017). Influence of Solar and Geomagnetic Activity on the Ionosphere over Bulgaria, C.R. Acad. Bulg. Sci., 70 (9), 1289-1296.

Pancheva, D. V., \& Mukhtarov, P. Y. (1996). A single-station spectral model of the monthly median F-region critical frequency. Annals of Geophysics, 39(4)

Rishbeth, H. (2006). F-region links with the lower atmosphere? J. Atmos. Sol.-Terr. Phys, 68(3-5), 469-478.

Schunk, R., \& Nagy, A. (2009). Ionospheres: physics, plasma physics, and chemistry, Cambridge university press. 


\title{
Certain Space Weather Conditions for a Supersubstorm Appearance
}

\author{
Despirak I.V. ${ }^{1}$, Lubchich A.A. ${ }^{1}$, Kleimenova N.G. ${ }^{2}$ \\ ${ }^{1}$ Polar Geophysical Institute, Apatity, Russia \\ ${ }^{2}$ Schmidt Institute of the Earth Physics RAS, Moscow, Russia \\ E-mail: despirak@gmail.com
}

\begin{abstract}
Analysis of the space weather periods associated with five events of the supersubstorms (SSS) was carried out. Two magnetic storms, on 8 September 2017 and 09 March 2012, have been studied. During these two storms, there were registered 5 supersubstorms. It was shown that the onsets of the SSS events were preceded by strong jumps in the dynamic pressure and density of the solar wind, which were observed against the background of a high solar wind speed. Besides, the high values of the Interplanetary Magnetic Field (IMF $\mathrm{B}_{\mathrm{T}}>10 \mathrm{nT}$ ) and high values of the southern $\mathrm{B}_{\mathrm{Z}}$ component of the IMF were also observed before the SSS occurrence. In additional, the values of the $\mathrm{B}_{\mathrm{Y}}$ component of the IMF were small positive or turned from negative to positive values.
\end{abstract}

\section{Introduction}

In the last 5 years there has been a rapid increase in interest in research of extremely intense substorms [Tsurutani et al., 2015; Haira and Tsurutani, 2018; Despirak et al., 2019, 2020a, 2020b; Nishimura et al., 2020]. The new type of very strong magnetic disturbances named as supersubstorms (SSS for shorthand) was first seen in the study by [Tsurutani et al., 2015, Haira et al., 2016] using the ground-based data from the SuperMAG magnetometers network. The supersubstorms were determined as very intense magnetic substorms with very large negative values of SML index $(\leq-2500 \mathrm{nT})$. This index is similar to the widely used AL-index, but derived from the 88 auroral SuperMAG stations [Gjerloev et al., 2012]. However, in our opinion, this definition of the SSS intensity (SML index threshold $\leq-2500 \mathrm{nT}$ ) should be extended, at least, up to SML values $\leq-2000 \mathrm{nT}$ [Despirak et al., 2020b], because this threshold proposed in the paper [Tsurutani et al., 2015] was an arbitrary one.

The SSS events as well as the classical substorms are typically observed during the main phase of a strong magnetic storm. However, the question arises whether the nature of the SSSs differs from classical substorms or not.

We found [Despirak et al., 2020a, 2020b] that in some SSS cases, the spatio-temporal development of the westward electrojet has a specific feature showing an almost global distribution in longitude from the evening to noon sector surrounding the Earth. The largest intensity of these SSS events was observed in the post-midnight sector. This activity was accompanied by the bay-like disturbances at dayside polar latitudes with the significantly reduced magnitude. The initial case studies of auroral disturbances during the SSS events showed that the development of the visible auroras is also nonstandard; the intense auroras were registered in the pre-midnight and morning sectors of magnetic local time (MLT) [Haira and Tsurutani, 2018].

Two examples of the SSS events are shown in Fig.1 as the time variations of the SML index on 11-12 April 2005, the moments of SSSs are pointed by the blue arrows. It is seen, that the SSSs were registered at 16:09 UT on 11 April (SML -2920 nT) and at 20:24 UT on 12 April 2001 (SML -2450 nT). 


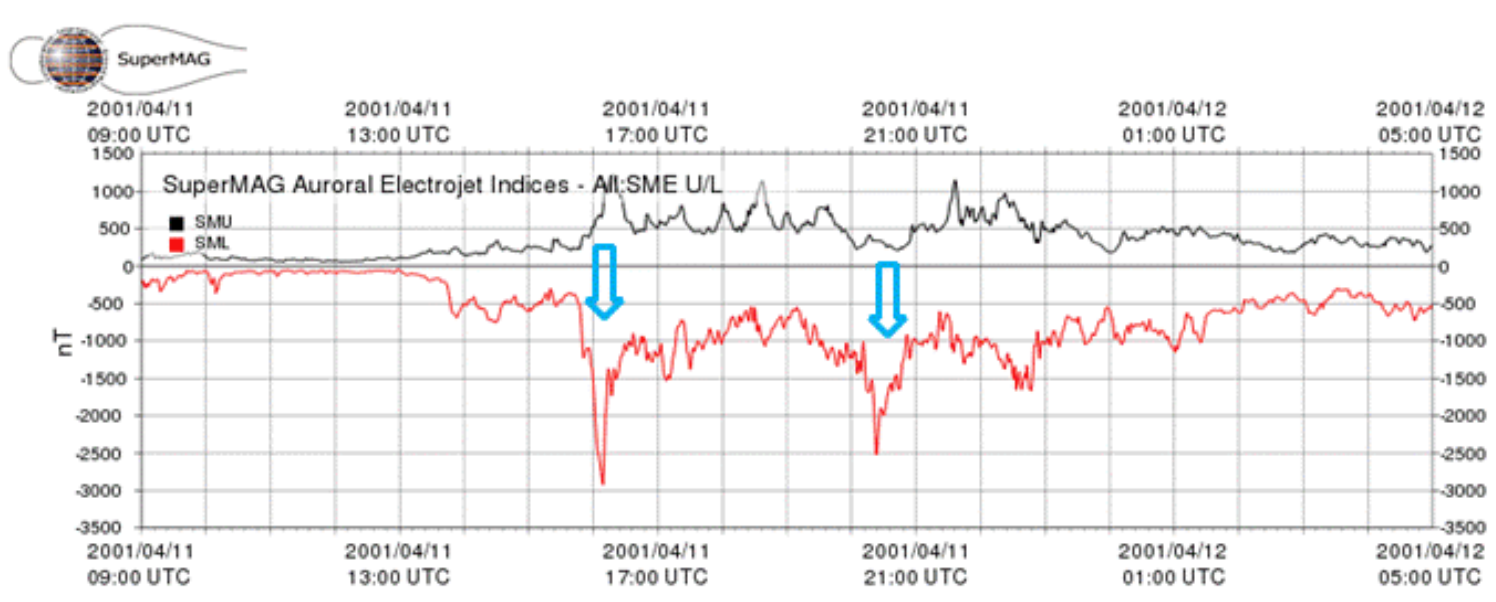

Fig.1. Variations of the indexes of geomagnetic activity from 09 UT on 11 April to 05 UT on 12 April 2001 by SuperMAG data are presented. The SMU index is shown at the top panel by the black line, SML index - at the bottom panel by the red line. Two moments of SSS observations are shown by the blue arrows.

Recently it was shown [Despirak et al., 2019] that SSSs occur during the certain types of the large-scale solar wind streams, namely, the SSSs are mainly observed during the approach to the Earth's magnetosphere of the solar wind magnetic clouds (MC) and SHEATH plasma compression regions. Thus, the SSS events are caused by interplanetary coronal mass ejections and are, in fact, unassociated with high-speed streams from coronal holes. Considering the solar wind types have a fairy long duration, there is a question if there are local drivers for supersubstorms.

The aim of our work is the search of the solar wind and interplanetary magnetic field (IMF) drivers of SSSs during two strong magnetic storms with SYM/H $150 \mathrm{nT}$. In our study, we considered the local behavior of the solar wind and IMF before the onset of the SSSs. For this purpose, the data of SuperMAG network and CDAWeb database were combined.

\section{Data}

We used the OMNI data base and the catalog of large-scale solar wind phenomena for determination of the solar wind types [Yermolaev et al., 2009]. These parameters were taken from the 1-min sampled OMNI data base of the CDAWeb (http://cdaweb.gsfc.nasa.gov/cgibin/eval2.cgi).

Extremely intense substorms (SSS) were determined by the SML index, based on the data from the magnetic ground-based stations of the SuperMAG network (http://supermag.jhuapl.edu/) and Scandinavian IMAGE network (http://space.fmi.fi/image/). Supersubstorms are defined as those events with the SML (AL) peak < -2000 nT.

\section{Observation results}

Here we present some results of our study of the magnetic storms on 8 September 2017 and 9 March 2012.

\section{a) Solar wind and IMF conditions for SSSs on 8 September 2017}

Figure 2 shows the several solar wind and IMF parameters: magnitude of magnetic field $\left(\mathrm{B}_{\mathrm{T}}\right), \mathrm{B}_{\mathrm{Y}^{-}}$and $\mathrm{B}_{\mathrm{Z}^{-}}$components of IMF, the flow speed and the dynamic pressure of the solar 
wind, SYM/H and SML indexes of geomagnetic activity. From the left panel of Fig. 2, it is seen that two consecutive structures in the solar wind were observed: SHEATH with EJECTA and SHEATH with Magnetic Cloud (MC). As result, there were two severe magnetic storms on 7 and 8 September 2017. The main phase of the first magnetic storm began with the arriving of the second SHEATH with the strong negative values of the IMF $\mathrm{B}_{\mathrm{Z}}$. The second magnetic storm was caused by MC with the negative IMF $\mathrm{B}_{\mathrm{Z}}$ component. Against the background of these two storms, two SSSs were registered, which were marked in Fig. 2 by the vertical, dotted blue lines. The first SSS $(\sim 3500 \mathrm{nT})$ was observed during second SHEATH, and the second SSS ( $2500 \mathrm{nT}$ ) was registered during MC. In the right panel of Fig. 2 shows the behavior of the solar wind parameters in more detail. It is seen that before both SSS occurrence, the local jump of the dynamic pressure was observed. The By component of the IMF was turned from the negative to positive values in the moment of the SSS occurrence. Besides, the high values of the southern $\mathrm{BZ}_{\mathrm{Z}}$ component of the IMF and the total magnetic field were also registered before the SSSs onsets.
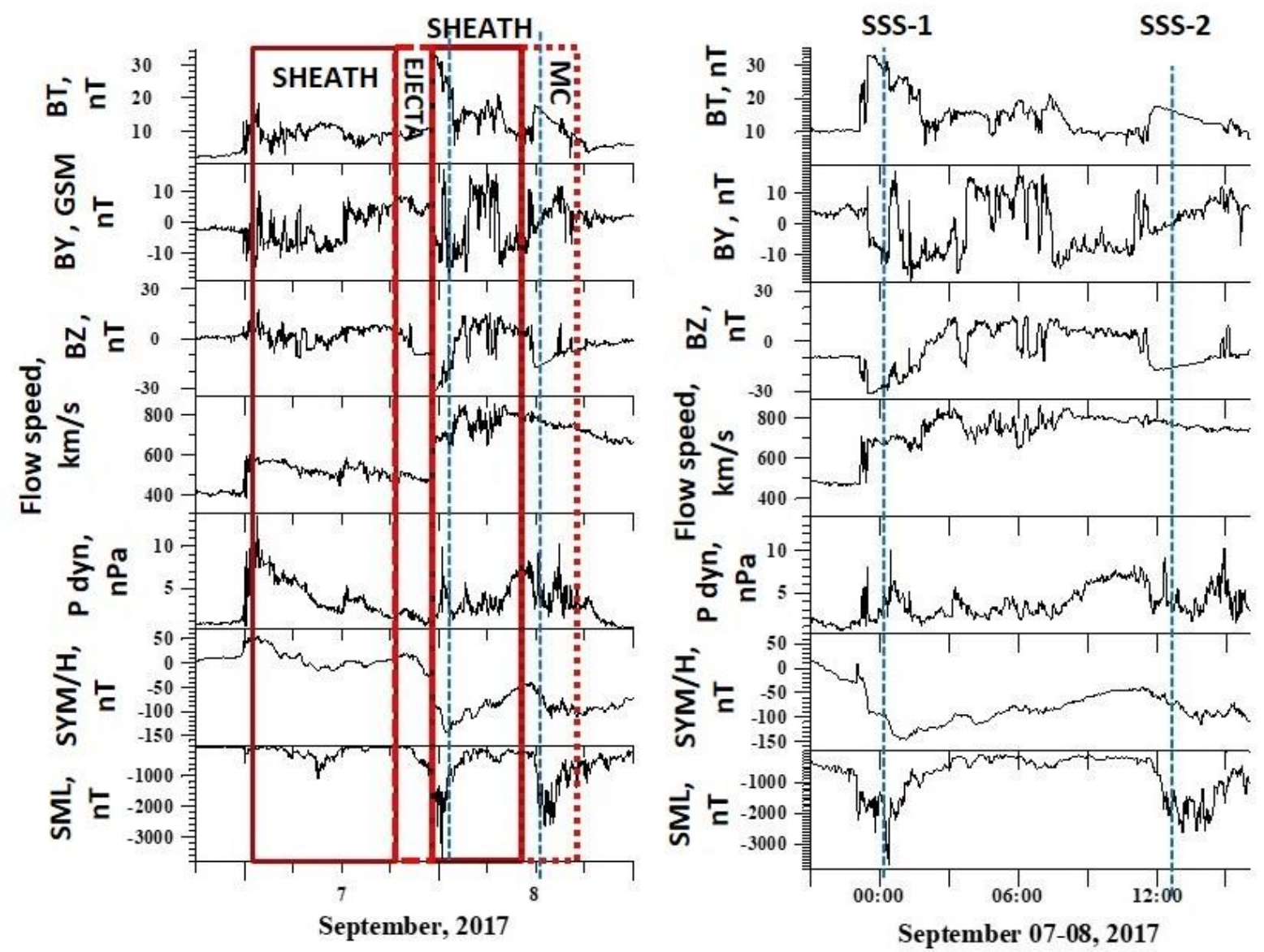

Fig.2. Variations of the solar wind and IMF parameters for two time periods: from 18 UT on 6 September to 24 UT on 8 September 2017 (a) and from 21 UT on 7 September to 16 UT on 8 September 2017 (b). The boundaries of the solar wind types are marked by the red rectangles: SHEATH - by the solid lines, MC and EJECTA - by the dotted lines. The moments of the SSS are shown by the vertical dotted blue lines.

\section{b) Solar wind and IMF conditions for SSSs on 9 March 2012}


Figure 3 shows the several solar wind and IMF parameters during the time period of 8 - 9 March 2012. The format of Fig. 3 is the same as Fig. 2. The boundaries of the magnetic cloud (MC) and the region of the compressed plasma (SHEATH) are indicated by the red rectangles: SHEATH - by the solid lines, MC- by the dotted lines.

It can be seen that at the beginning of MC, an increase in the southward IMF component ($14 \mathrm{nT}$ ) was observed, which occurred against the background of the high solar wind speed (700$750 \mathrm{~km} / \mathrm{s}$ ), which led to the development of a magnetic storm. The intensity peak of this storm $(\mathrm{SYM} / \mathrm{H} \sim-140 \mathrm{nT})$ was recorded at $\sim 08 \mathrm{UT}$ on 9 March. At the main phase of the magnetic storm, at 06-16 UT, a sharp increase in the module of the SML index was observed in the form of a sequence of substorms, it was the complex pattern of the disturbances from which it is difficult to distinguish an individual substorm. In Fig. 3, at least 4 minima in the SML index are seen: $\sim-2800 \mathrm{nT}, \sim-2500 \mathrm{nT}, \sim-2370 \mathrm{nT}$, and $\sim-2200 \mathrm{nT}$, three of them will be considered in this work as SSS events: at 06:55 UT (SSS-1), at 09:19 UT (SSS-2) and at 11:57 UT (SSS3). In Fig. 3 these events are marked by the vertical blue dashed lines and designated as SSS-1, 2 , 3. It is seen that all these SSS events were observed during MC, in the main phase of the magnetic storm, closely to the minimum of the SYM/H index.

In Fig. 3b, the behavior of the solar wind parameters shows in more details. It is seen that before occurrence of each SSS event, there was registered a local jump of the solar wind dynamic pressure. Note, that these jumps often occurred under the high values of the solar wind speed. In additional, before the SSS-1, the BY component of the IMF was turned from the negative to positive values and the values of this component were small positive before both the SSS-2 and SSS-3. Besides, the high values of the southern $\mathrm{B}_{\mathrm{Z}}$ component of the IMF and the total magnetic field were also registered before each of the SSS onsets.

\section{Discussion}

Thus, our analysis of the space weather conditions before and during the occurrence of five SSS events during two strong magnetic storms (on 8 September 2017 and 9 March 2012) showed that there were the specific conditions in the solar wind and IMF leading the generation of huge intensity substorms (so called supersubstorms) with the magnitudes higher $2000 \mathrm{nT}$.

We found that all five SSS events under consideration were observed during the main phases of the magnetic storms, four of the SSS events were observed during the magnetic storm associated with MC, and one SSS event was observed during the magnetic storm associated with SHEATH. All five studied SSS events were observed under high values of the solar wind velocity (Vsw $\sim 700 \mathrm{~km} / \mathrm{s})$ and strong total magnetic field $\left(\mathrm{B}_{\mathrm{T}}>10 \mathrm{nT}\right)$.

Moreover, all SSS events were generated only after the occurrence of the very strong values of the southern $\mathrm{B}_{\mathrm{Z}}$ component of the IMF (up to $-30 \mathrm{nT}$ ). That fact indicates a huge input solar wind energy into the magnetosphere which is proportional to the product of $\mathrm{Vsw}$ by $\mathrm{B}_{\mathrm{T}} \mathrm{IMF}$ or $\mathrm{Vsw}^{*}\left(\mathrm{BzIMF}^{2}+\mathrm{ByIMF}^{2}\right)^{1 / 2}[$ Kan and Lee, 1979; Troshichev et al., 2014].

We found also that before each of the SSS events, there was observed a jump of the solar wind dynamic pressure. This jump was strong in the magnetic storm on 8 September 2017 reaching $\sim 10 \mathrm{nPa}$ and much smaller in the magnetic storm on 9 March 2012. We suppose that the sudden compression of the magnetosphere by a sharp jump of the solar wind dynamic pressure could be local driver of a supersubstorm generation. The substorm onset triggering by interplanetary shocks was previously discussed in the paper [Zhou and Tsurutani, 2001]. 

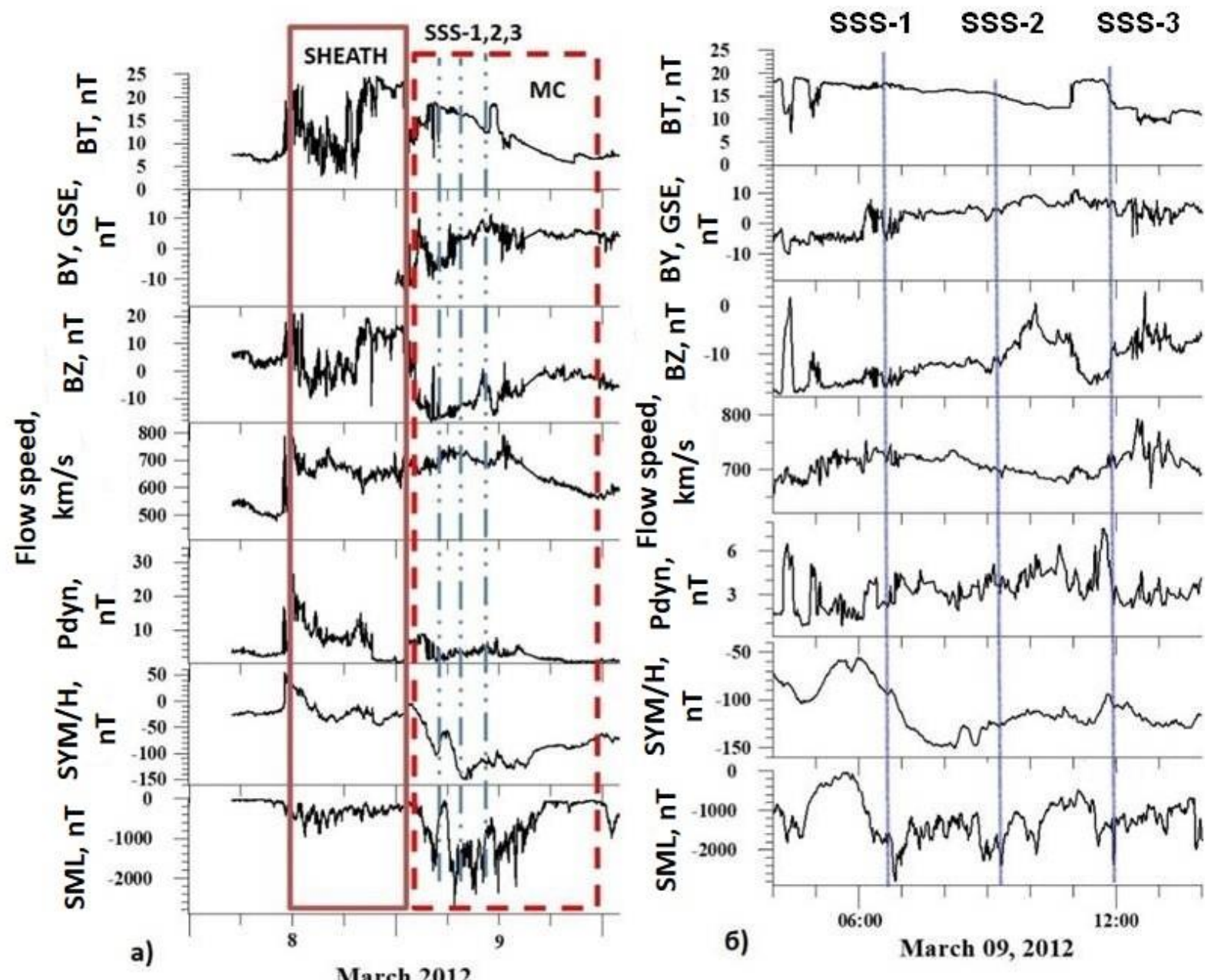

Fig.3. Variations of the solar wind and IMF parameters for two time periods: from 00 UT on 8 March to 24 UT on 9 March 2012 (a) and from 03 tol4 UT on 9 March 2012 (b). The boundaries of the solar wind types are marked by the red rectangles: SHEATH - by the solid lines, MC - by the dotted lines. The moments of SSSs are shown by the blue vertical dotted (a) and solid (b) lines.

\section{Conclusions}

We considered the space weather conditions for occurrence of the supersubstorms (SSS) during two magnetic storms. The analysis of five SSS events shown that before SSS were registered some local features in the solar wind and IMF behavior:

1) the local jump of the dynamic pressure

2) the high values of the southern $\mathrm{Bz}$ component of the IMF (up to $-30 \mathrm{nT}$ ) and the total magnetic field $\mathrm{B}_{\mathrm{T}}(\sim>10 \mathrm{nT})$.

3 ) the By component of the IMF was small positive or turned from negative to positive values We may suggested that the most probable source for the appearance of supersubstorms may be an increase in the dynamic pressure by the background of the southward direction of the interplanetary magnetic field and the solar wind speed.

\section{References}

Despirak, I.V., Lyubchich, A.A., N.G. Kleimenova, N.G. (2019). Supersubstorms and conditions in the solar wind, Geomag. Aeron., 59, no 2, 170-176. 
Despirak, I.V., Kleimenova, N.G. Gromova, L.I., Gromov, S.V., Malysheva, L.M. (2020a). Supersubstorms during storms of September 7-8, 2017, Geomag. Aeron., 60, no 3, 308-317.

Despirak, I., Lubchich, A., Kleimenova, N. Gromova, L., Gromov S., Malysheva L. (2020b). Supersubstorms during two strong magnetic storms, Proceedings of International Conference "Atmosphere, ionosphere, safety", Kaliningrad, 2020, 97-99.

Gjerloev, J.W. The SuperMAG data processing technique (2012). J. Geophys. Res., V. 117(A9), A09213. https://doi.org/10.1029/2012JA017683

Hajra, R., Tsurutani, B.T., Echer E., Gonzalez, W.D., Gjerloev, J.W. (2016). Supersubstorms (SML < -2500 nT): Magnetic storm and solar cycle dependences, J. Geophys. Res., 121, 7805-7816, doi: 10.1002/ 2015 JA021835.

Hajra, R., Tsurutani, B.T. (2018). Interplanetary shocks inducing magnetospheric supersubstorms (SML $<-2500$ nT): Unusual auroral morphologies and energy flow, Astrophys. J., 858, no. 2, id 123. https://doi.org/10.3847/1538-4357/aabaed.

Kan, J. R., Lee, L. C. (1979). Energy coupling function and solar wind-magnetosphere dynamo, Geophys. Res. Lett., 6(7), 577-580, 1979.

1. Nishimura, Y., Lyons, L.R., Gabrielse, C., Sivadas, N., Donovan, E.F., Varney, R.H., Angelopoulos, V., Weygand, J.M., Conde, M.G., Zhang, S.R. (2020). Extreme magnetosphere-ionosphere-thermosphere responses to the 5 April 2010 supersubstorm, J. Geophys. Res. https://doi.org/10.1029/2019JA027654

2. Troshichev, O. A., Podorozhkina, N. A., Sormakov D. A., Janzhura A. S. (2014), PC index as a proxy of the solar wind energy that entered into the magnetosphere: Development of magnetic substorms, J. Geophys. Res. Space Physics, 119, 6521-6540, doi:10.1002/2014JA019940

Tsurutani, B.T., Hajra, R. Echer, E., Gjerloev, J.W. (2015). Extremely intense (SML $\leq-2500 \mathrm{nT}$ ) substorms: Isolated events that are externally triggered? Ann. Geophys., 33 (5), 519-524.

Yermolaev, Yu.I., Nikolaeva, N.S., Lodkina, I.G. et al.. (2009). Catalog of large-scale solar wind phenomena for the period 1976-2000, Cosmic Res. (Engl. Transl.), 47, 81-94.

Zhou, X.-Y., Tsurutani, B. T. (2001). Interplanetary shock triggering of nightside geomagnetic activity: Substorms, pseudobreakups, and quiescent events, J. Geophys. Res., 106, 18957-18967. 


\title{
Local and Global Longitudinal Geomagnetic SC Effects at High- Latitudes: Case Study of the Interval of March 07-15, 2012
}

\author{
Gromova L.I. ${ }^{1}$, Kleimenova N.G. ${ }^{2}$, Gromov S.V. ${ }^{1}$, Malysheva L.M. ${ }^{2}$ \\ ${ }^{1}$ IZMIRAN, Moscow, Russia \\ ${ }^{2}$ Schmidt Institute Physics of the Earth RAS, Moscow, Russia \\ e-mail: gromova@izmiran.ru
}

\begin{abstract}
The geomagnetically active interval of March 7-17, 2012 was the first long interval with high geomagnetic activity in the ascending phase of Solar Cycle 24. It was selected by SCOSTEP for intensive study of the space weather features. Four different magnetic storms with SC have been observed during this time interval It was found that this storm development was accompanied by an enhancement of the magnetic activity at the high latitudes of the Earth. Here we present the results of our study of the SC longitudinal effects on the high-latitude geomagnetic disturbances. The analysis of the ground-based magnetic observations showed that the longitudinal distribution of the high-latitude geomagnetic effect of the SC events depends on the Interplanetary Magnetic Field (IMF) direction as well as on the amplitude of a jump in the solar wind dynamic pressure $(P s w)$.
\end{abstract}

\section{Introduction}

The overview of major solar, interplanetary, magnetospheric, and ionospheric features of the geomagnetically active interval of March 7-17, 2012 was reported in [Tsurutani et al., 2014, Gopalswamy et al, 2015]. These authors particularly discussed the interplanetary conditions in this interval leading to the developing of the magnetic storms on 7, 9, 12, and 15 March with the peaks of the $S_{y m H_{\min }}$ of $-98 \mathrm{nT},-148 \mathrm{nT},-75 \mathrm{nT}$, and $-79 \mathrm{nT}$, respectively, associated with the arriving of the magnetic clouds (MC). They found that there were strong interplanetary shocks preceding these magnetic storms. Tsurutani\& Zhou (2003) found that under the southward IMF, such shocks could cause the substorm occurrence at auroral latitudes.

However, the global scale high-latitude geomagnetic activity in this interval remained outside the scope of these papers. The aim of our paper is to show the global high-latitude geomagnetic disturbances associated with the interplanetary shocks observed as four SC events during the interval of March 7-15, 2012

\section{Observations and discussion}

Figure 1 shows the development of the global $(\mathrm{SymH})$ and auroral $(A L)$ geomagnetic activity, and the IMF the and solar wind conditions as variations of the IMF $B z$ component and the solar wind dynamic pressure during the interval under study during the interval of March 7-15, 2012

Four magnetic storms with SC have been observed during this time interval. They are shown by arrows on the SymH index panel. SC-1 occurred under the strong negative IMF $B z$, during SC-2 the IMF $B z$ was strong positive. During SC-3, SC-4 the IMF $B z$ changed from negative to positive, the amplitudes of jump of the solar wind dynamic were different from each other that should caused different magnification of SC events as well. 


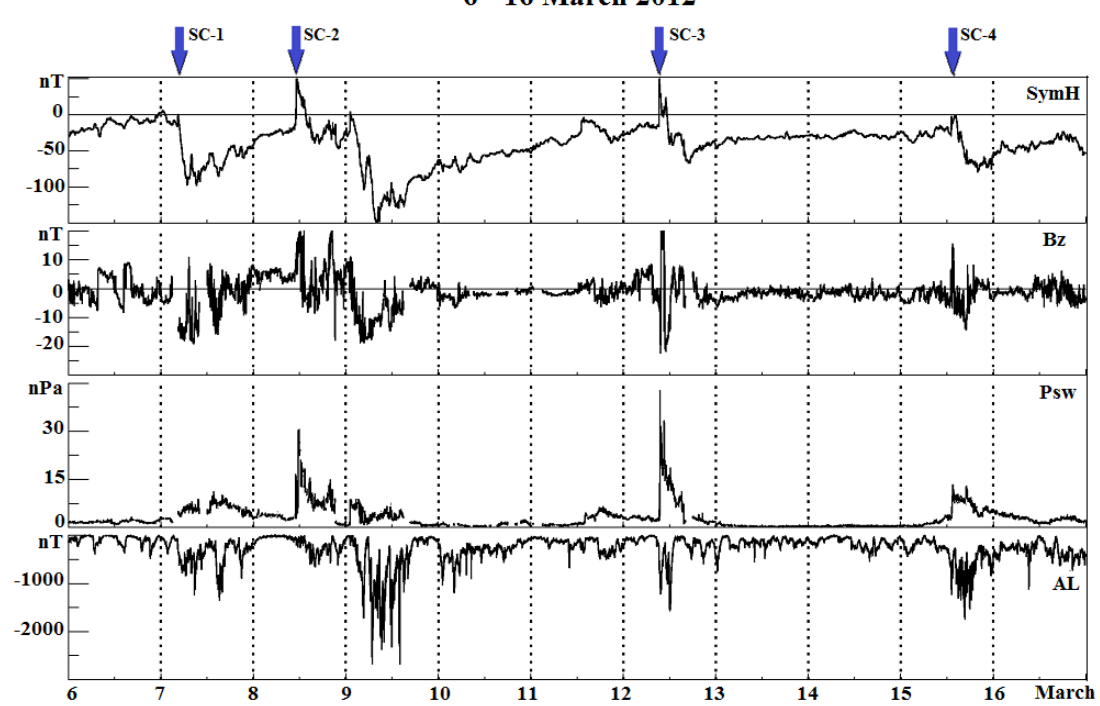

Fig. 1. Interval of March 6-16,2012. Variations of the index of a magnetic storm intensity SymH, IMF $B z$ component, solar wind dynamic pressure Psw, and index of auroral activity AL Blue arrows point the UT moment of the SCs arrival.

\section{SC-1 event}

The IMF $B z$ was stable strong negative and isn't changed during 2,5 hours after SC-1. The amplitude of the Psw jump was small (Fig.2a).

\section{March 2012}
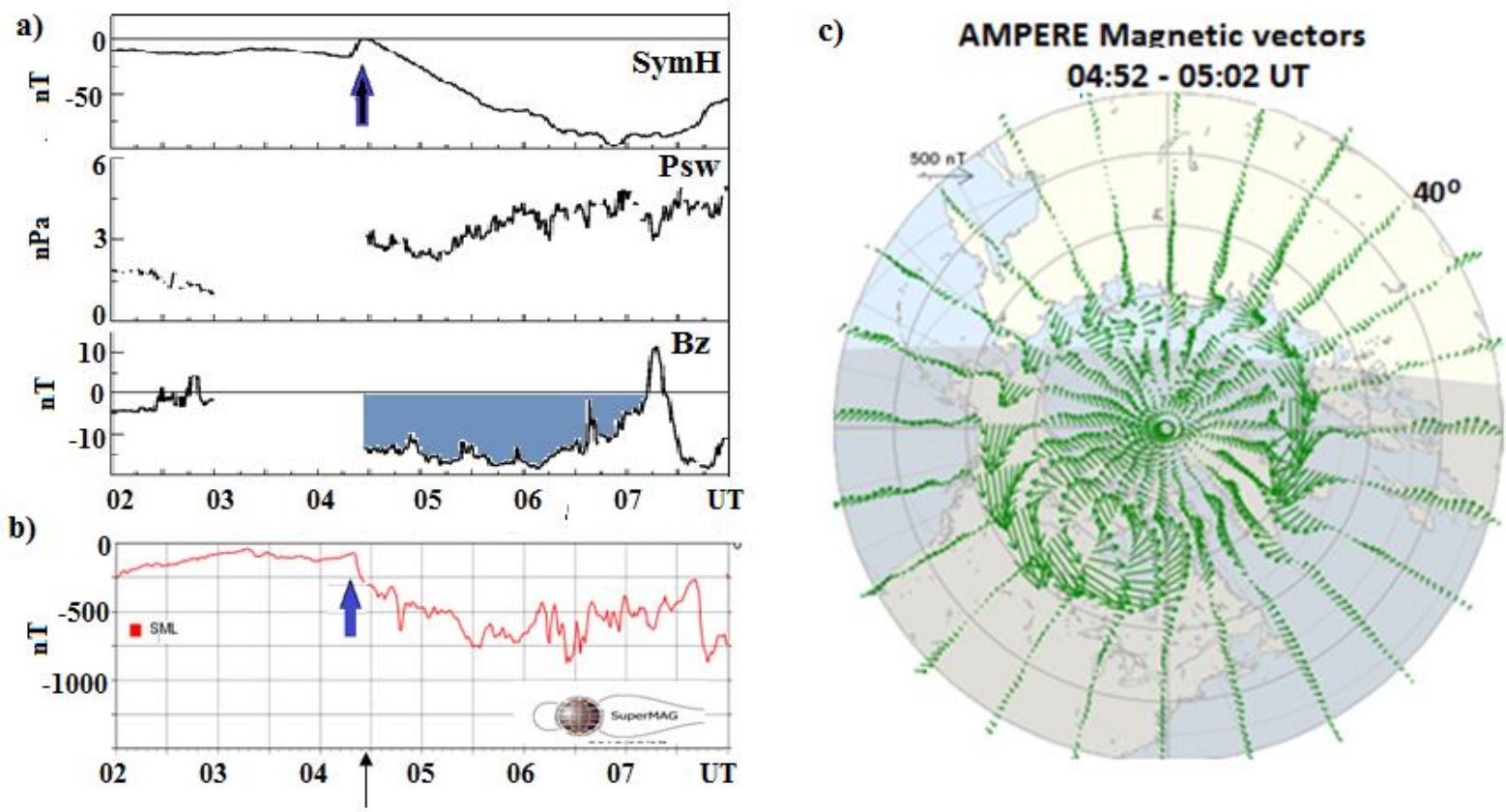

Fig.2. SC-1 on 7 March 7, 2012: (a) Variations of the SymH index, Psw and the IMF Bz; the interval of the negative IMF Bz are marked by blue; thick arrow shows the SC-1 time. (b) SML index of the auroral activity. (c) The AMPERE magnetic field perturbations over the highlatitude region as the result of spherical harmonic analysis; location of some polar and auroral stations is shown by circles. Arrow on the UT-time axis of (b) points the map moments.

Negative strong Bz and the small jump of Psw (SC-1) initiated strong beginning of the magnetic storm that always accompanied by a sequence of substorms (Fig.2b) that is seen on 
SML index panel. AMPERE map shows the geomagnetic disturbances at auroral latitudes (Fig. 2c), no noticeable geomagnetic disturbances were observed at the polar latitudes.

\section{SC-2 event}

The IMF $B z$ was stable strong positive and isn't changed during long time interval before and after the PSW jumps. The first $P s w$ jump wasn't large (SC-2). Later the IMF $B z$ graduallyincreasing, and the $P s w$ sharply jumped to large value (Fig.3 a).

Positive Bz and large jump of Psw led to magnetic bays observed at the polar stations of the dayside, (NAL, HOR), morning (THL) and night (RES) sectors (Fig.3b,c). But the auroral activity was weak (Fig. 3d).

a)

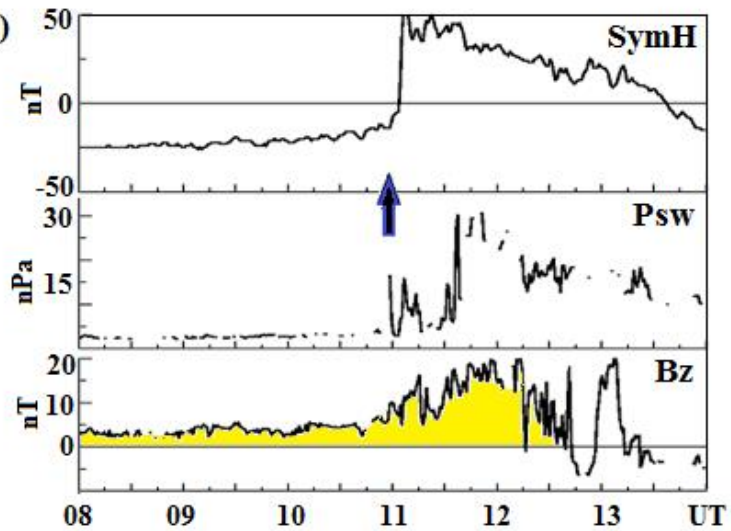

\section{March 2012}

c)

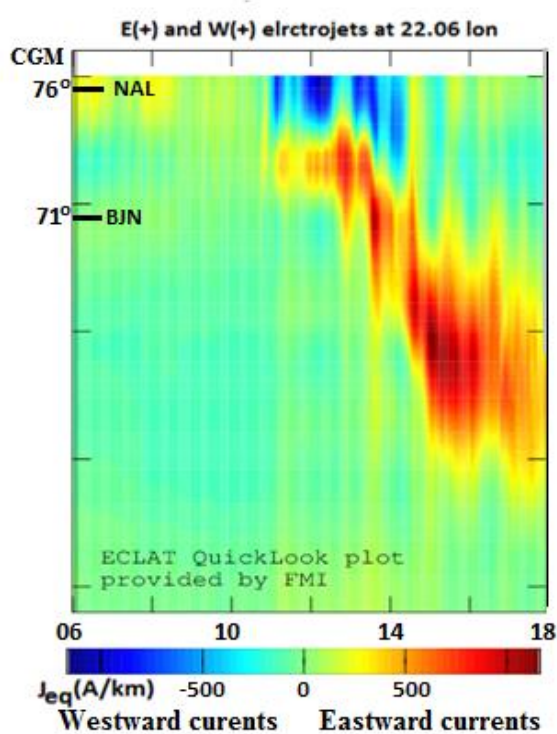

d)

AMPERE Magnetic vectors 11:58 - 12:08 UT

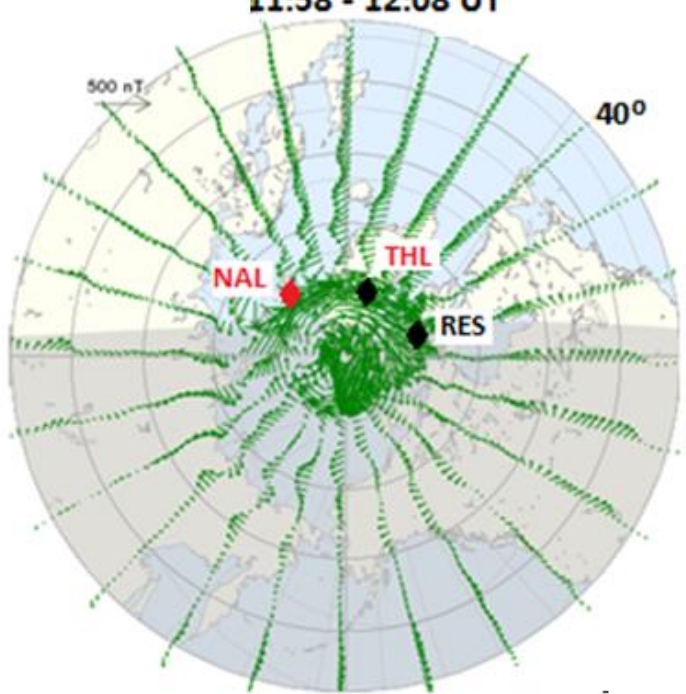

Fig.3. SC-2 on March 8, 2012: (a) Variations the index SymH, Psw and the IMF Bz; thick arrow the time of SC-2 arrival. (b) IMAGE and Intermagnet magnetograms of the high latitude stations, triangles show the local geomagnetic time at the 09 UT. (c) Equivalent ionospheric currents by ECLAT model. (d) The AMPERE distribution of the magnetic field as it is shown in Fig. $2 c$. 


\section{SC-3 event}

The negative IMF $B z$ was stable in long interval before the large Psw jump (SC-3). Later Bz briefly changed from negative to strong positive and remained stable during $\sim 1$ hour, at the same time $P s w$ sharply jumped to very large value. Then the IMF $B z$ returned to negative value and was stable about 2,5 hours. (Fig. 4a).

a)

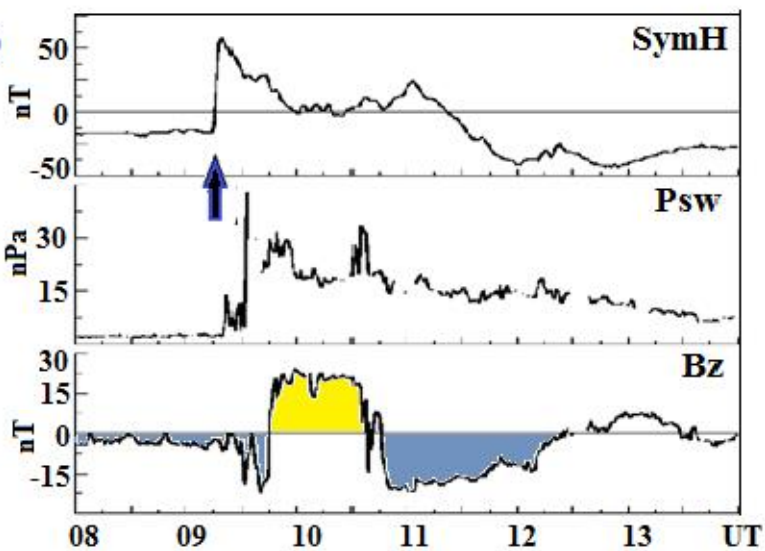

b)
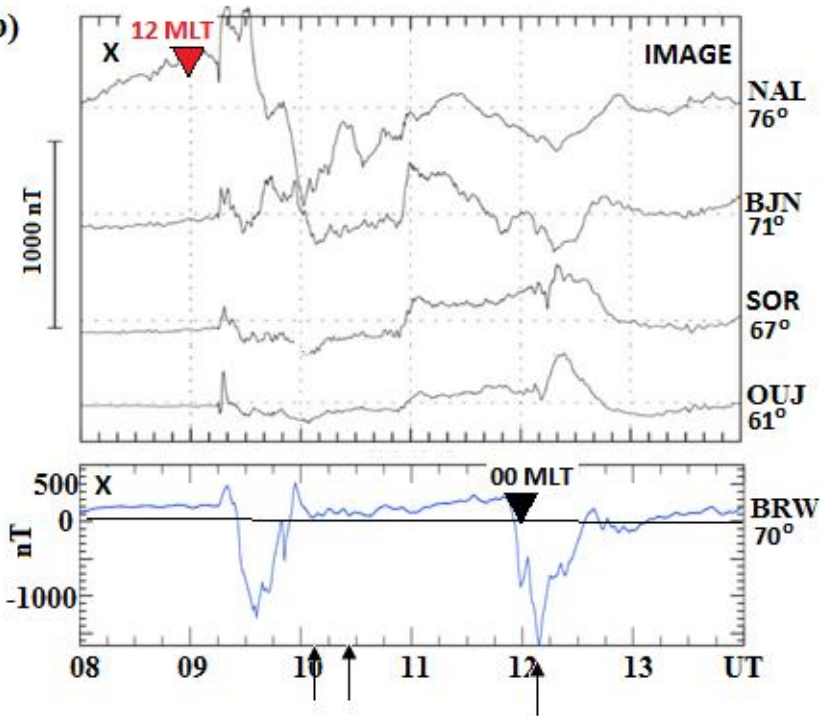

12 March 2012

c)

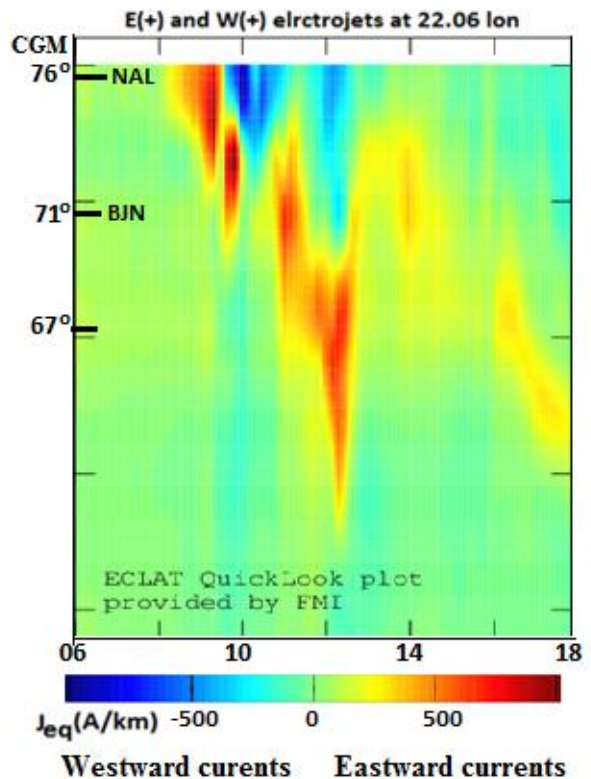

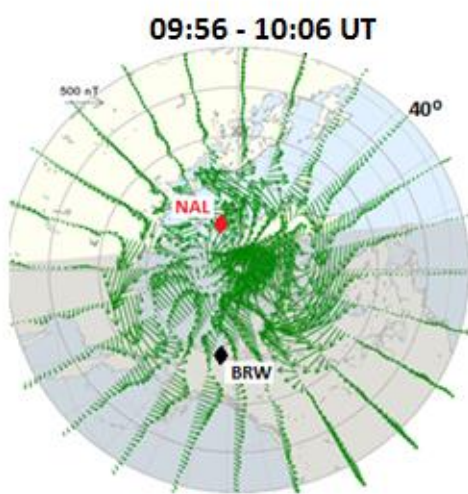

AMPERE magnetic vectors
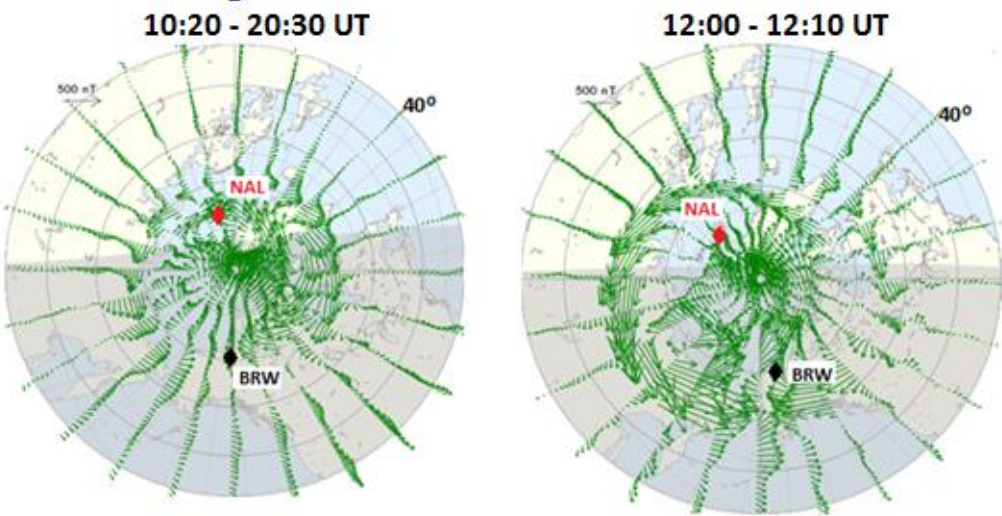

Fig.3. SC-2 on March 8, 2012: (a) Variations the index SymH, Psw and the IMF Bz; thick arrow the time of SC-2 arrival. (b) IMAGE and Intermagnet magnetograms of the high latitude stations, triangles show the local geomagnetic time at the 09 UT. (c) Equivalent ionospheric currents by ECLAT model. (d) The AMPERE distribution of the magnetic field as it is shown in Fig. $2 c$. 
The long interval of the negative IMF Bz preceding the SC-4 and large jump of Psw led to the development of global substorms, which were observed in all sectors of the polar and auroral latitudes. With the IMF $B z$ sharp changing to large positive values, the substorm in auroral latitudes stopped, and the polar disturbances are observed in the daytime sector only (Fig. 4b, c, d).

\section{SC-4 event}

As in SC-3 event, the negative IMF $B z$ was stable in long interval before the small Psw jump (SC-4). Then $\mathrm{Bz}$ briefly changed from negative to positive and gradually increasing, remained stable during $\sim 1$ hour, at the same time $P s w$ gradually reached large value. 3)Then the IMF Bz returned to negative value remained stable in a long time (Fig.5a).

\section{March 2012}

a)

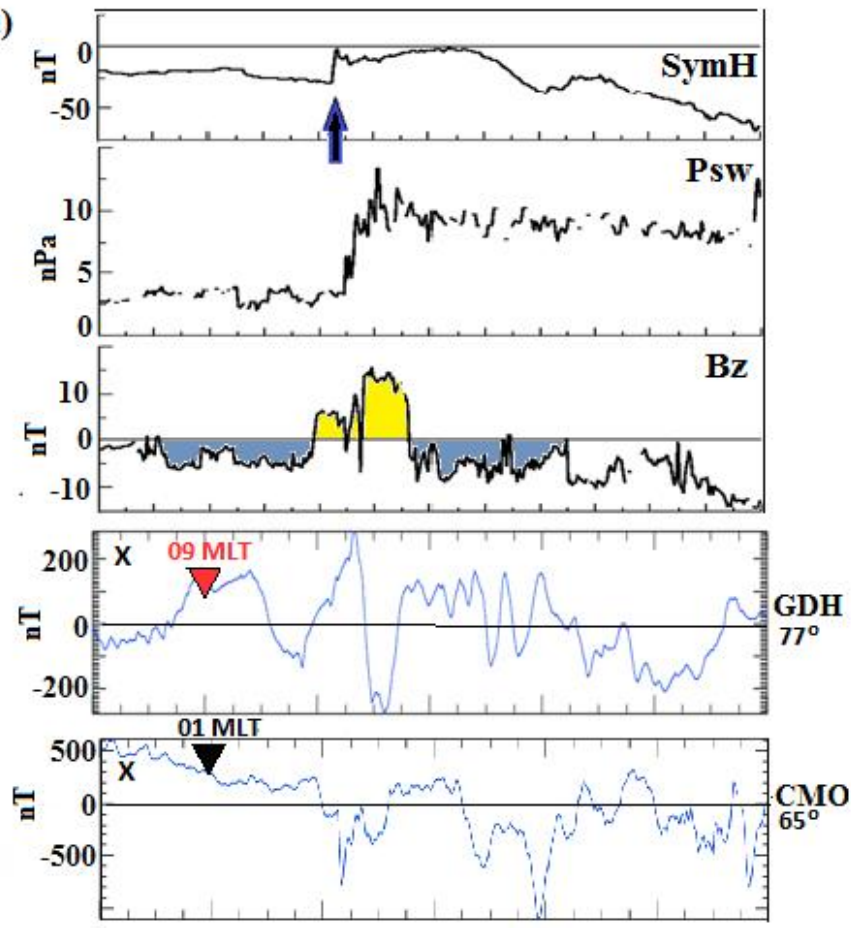

b)

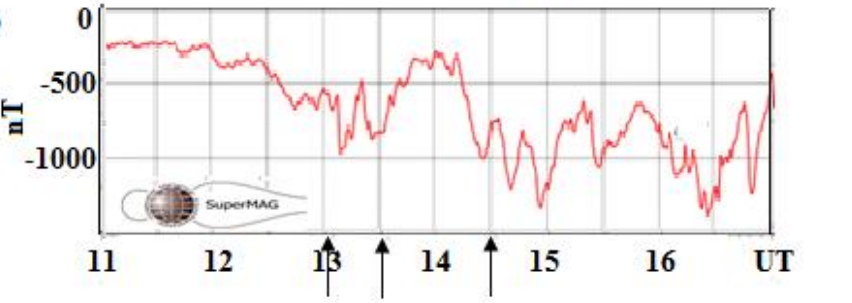

\section{c) AMPERE magnetic vectors}
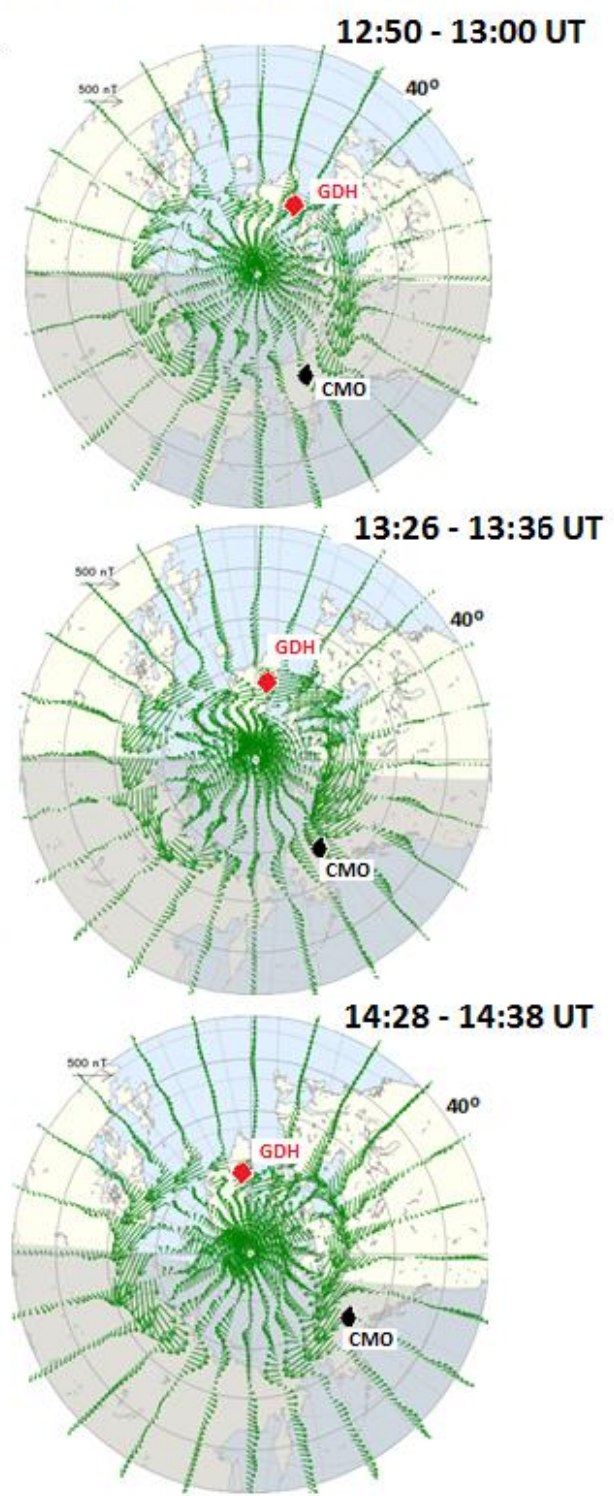

Fig. 5. SC-4 on March 15, 2012: (a) Variations the SymH index, the IMF Bz and Psw; thick arrow shows the time of SC-4 arrival. (b) Intermagnet magnetograms of the high latitude stations, triangles point the local geomagnetic noon (red) and midnight(black). (c) The AMPERE distribution of the magnetic field as it is shown in Fig.2c. 
The long interval of the negative IMF Bz preceding the arrival of SC-4 event and small jump of the PSW under positive IMF $B z$ did not change common signature of the geomagnetic activity at the high latitude region. The sharp small Psw jump under the positive IMF Bz led to development of the magnetic activity in the dayside sector of the polar latitudes without any auroral activity. The IMF $B z$ changing from positive to negative leds to development of the auroral activity with weak disturbances in the polar region. (Fig.5b.c.d).

\section{Conclusion}

The magnification of four SC events during the interval of March 7-15, 2012 has been studied.

The analysis of the ground-based magnetic observations showed that the longitudinal distribution of SC high-latitude geomagnetic effect depended on the interplanetary magnetic field (IMF) direction as well as on the amplitude of a jump in the solar wind dynamic pressure (Psw).

We found that under the positive IMF Bz and the large amplitude ( 15 nPa) of the Psw jump, the high-latitude SC effects were observed around the pole in all longitudes at the polar latitudes with weak auroral activity. But under a small Psw jump ( 3-4 nPa), this effect was observed only in the dayside sector of the polar latitudes.

However, under the negative IMF Bz and the large Psw jump, the high-latitude SC effects were observed as a development of the substorms in the global scale, i.e. in all longitudes of the auroral and polar latitudes, but under the small Psw jump, this effect was observed only at the night-side auroral latitudes.

\section{Data}

Our study is based on: OMNI database of the Interplanetary Magnetic field (IMF) data of the 1-min resolution (http://omniweb.gsfc.nasa.gov); ground-based magnetic data of the IMAGE magnetometer chain (http://space.fmi.fi/image/) and the global network of observatories INTERMAGNET (http://www.intermagnet.org/)); SML-index derived as analog of AL-index from the magnetometer data of all available ground magnetometer stations at geomagnetic latitudes between $40^{\circ}$ and $80^{\circ}$ provided by the global SuperMAG network (http://supermag.jhuapl.edu/indices); the AMPERE data, based on the ionospheric magnetic measurements on 66 low-altitude globally distributed Iridium communication satellites (http://ampere.jhuapl.edu/products/plots); the model ECLAT of the equivalent ionospheric currents (http://space.fmi.fi/image/).

\section{References}

Gopalswamy, N., Tsurutani, B., Yan, Y.(2015), Short-term variability of the Sun-Earth system: an overview of progress made during the CAWSES-II period, Progress in Earth and Planetary Science, 2, 13.

https://doi.org/10.1186/s40645-015-0043-8

Tsurutani, B.T., and Zhou, X.-Y. (2003), Interplanetary shock triggering of substorms: WIND and POLAR, Adv. Spa. Res., 31, 1063, 2003. https://doi.org/10.1016/S0273-1177(02)00796-2

Tsurutani, B, Echer, E, Shibata, K, Verkhoglyadova, O, Mannucci A, et al. (2014), The interplanetary causes of geomagnetic activity during the 7-17 March 2012 interval: a CAWSES II overview. J. Space Weather Space Clim., 2014, 4, A02. https://doi.org/10.1051/swsc/2013056 


\title{
Substorm Activity During the Geomagnetic Storm on 25 August 2018
}

\author{
Guineva V., Werner R. \\ Space Research and Technology Institute BAS, Stara Zagora Department, Bulgaria \\ E-mail:v_guineva@yahoo.com
}

\begin{abstract}
.
The substorms originated during the geomagnetic storm on 25 August 2018 have been studied. This storm developed unexpectedly at the very end of the 24 solar cycle. It was associated with a CME, related to a filament eruption on 20 august 2018. A magnetic cloud (MC) reached the Earth, followed by a corotating interaction region (CIR) in the solar wind. The Bz component of the interplanetary magnetic field (IMF) fold sharp and stayed negative for about 18 hours. This storm appears to be the third by minimal SYM/H value after the storms on 15 March and 22 June 2015. SYM/H reached -206 nT in 7:11 UT on 26 August 2019. The storm was a strong one, $\mathrm{K}_{\mathrm{pmax}}=7^{+}$in 6-9 UT on 26 August 2018.

Several substorms were observed during the main and recovery phase of the storm. The occurrence and spreading of the magnetic substorms were studied by IMAGE and INTERMAGNET networks data. For the solar wind and Interplanetary Magnetic Field parameters data from the OMNI data base have been used. SML index was taken from the SuperMag database, and the $\mathrm{K}_{\mathrm{p}}$ index - from the Kioto Center for geomagnetism website. The substorms development was examined. The substorms latitudinal extent in the range $92^{\circ}-108^{\circ}$ CGMlon was studied. The magnetic substorms were observed also at middle latitudes as positive magnetic bays. The peculiarities of the substorms and their display at mid-latitudes have been analysed.
\end{abstract}

\section{Introduction}

Substorms are a typical event at auroral latitudes. It is well known that during the substorm expansion phase, the westward electrojet moves fast poleward, usually by consecutive jumps, and under highly enhanced magnetic activity could reach latitudes well above the typical location of the night side auroral oval [e.g., Akasofu S.-I., 1964; Kisabeth J.L. et al., 1974; Wang H. et al., 2005; Despirak I.V. et al., 2008; Tanskanen E.I. et al., 2009, Clausen et al., 2013; Despirak I. V. et al., 2014]. It should be noted also that under disturbed conditions, at increased magnitude of the IMF negative $\mathrm{B}_{\mathrm{Z}}$ component, the equatorward boundary of the oval shifts down to about $50^{\circ}$ geomagnetic latitude, while its poleward boundary extends to higher latitudes than the usual ones, i.e. the so called "expanded oval" is formed [e.g. Feldstein Y.L. et al., 1967]. It was found out, that the most intensive substorms occur on expanded auroral oval [Clausen L.B.N. et al., 2013]. It is well known also, that in such conditions substorms can be observed at middle and even low latitudes as positive magnetic bays [e.g., McPherron et al., 1973]. Akasofu et al., (1965) explained the positive bays as a result of the low-latitude return currents from the westward electrojet. Later Akasofu and Meng (1969) and Meng and Akasofu (1969) explained the positive bays as a result of the field aligned currents. McPherron R.L. et al., (1973) developed further the concept of the current system, responsible for the substorm onset and expansion - the Substorm Current Wedge (SCW). By the up-to-date views, the positive bays at mid-latitudes are observed in the substorm expansion phase and are caused by the SCW [McPherron R.L. et al., 2017, 2018].

The aim of this paper is to study the magnetic substorms development under highly disturbed interplanetary and geomagnetic conditions, during the strong magnetic storm on 25 August 2018, and analyze their appearance at middle latitudes. 


\section{Data used}

The data sources used for the study of magnetic substorms are listed in detail in Guineva et al, (2019).

We used the magnetic data from the IMAGE and INTERMAGNET networks. From the IMAGE set, we considered data from the meridional chain stations Polesie (PPN) - Ny Ålesund (NAL), situated in the longitudinal range $98^{\circ}-112^{\circ} \mathrm{CGMLon}$, and covering the latitudinal range from $47^{\circ}$ to $75^{\circ} \mathrm{CGM}$ lat. The chosen INTERMAGNET stations are in the longitudinal range of $92^{\circ}-104^{\circ}$ CGMLon, from $35^{\circ}$ to $64^{\circ}$ CGMLat. The westward electrojet development was estimated by the time evolution of the equivalent ionospheric currents, computed by the Finish Meteorological Institute (FMI) on-line tool for $22.06^{\circ}$ lon. ( 112 ${ }^{\circ}$ CGMLon).

The solar wind and Interplanetary Magnetic Field parameters data were taken from the OMNI data base, and the data for the structures in the solar wind - from the catalog of largescale solar wind phenomena [Yermolaev, Yu.I. et al., 2009]. The SML index was provided from the SuperMag database, and the $\mathrm{K}_{\mathrm{p}}$ index - from the Kioto Center for geomagnetism website.

\section{Interplanetary and geomagnetic conditions on 25-29 August 2018}

Fig.1 illustrates the interplanetary and geomagnetic conditions in the time period 25-29 August 2018. From up to down the following quantities are displayed: the magnitude of the interplanetary magnetic field (IMF) $B_{T}$, the IMF $B_{Z}$, the flow velocity $V$, the flow velocity $X$ component $\mathrm{V}_{\mathrm{X}}$, the plasma density, temperature (T), pressure (P), and the SML, SYM/H and $K_{P}$ geomagnetic indices. A filament eruption was registered on the Sun on 20 August 2018, and a related coronal mass ejection (CME) propagated towards the Earth. A magnetic cloud (MC) reached the Earth in 14 UT on 25.08.2018, followed by a high speed stream (HSS). The structures in the solar wind during the examined period are indicated in Fig.1. The Sheath in front of MC initiated a strong storm ( $\mathrm{k}_{\mathrm{pmax}}=7^{+}$in 6-9 UT on 26 August 2018). The initial storm phase began at 6:30 UT, 25.08.2018, and the main phase - from 17:40 UT on 25.08.2018. This storm was the third by minimal SYM/H value after the storms on 15 March and 22 June 2015. SYM/H reached -206 nT in 7:11 UT on 26 August 2019. Ap index was 67.

\section{Magnetic substorms}

During the geomagnetic storm, 6 substorms were observed in the examined area $\left(\sim 93^{\circ}\right.$ $112^{\circ}$ CGMLon, $35^{\circ}-75^{\circ}$ CGMLat) by magnetic data. 3 substorms occurred during the main storm phase, and 3 - during the recovery phase. In all cases, positive magnetic bays were present at middle latitudes. 3 substorms were considered in details: 2 substorms on 25 August 2018, in the beginning of the main storm phase, and one substorm on 27 August 2018, in the late recovery phase. The substorms development is shown in Fig.2 and Fig.3. In Fig. 2, the equivalent ionospheric currents (upper panel) and the X-component of the magnetic field from the IMAGE latitudinal chain PPN-NAL (bottom panel) are given for the substorms on 25 August 2018 (left panels) and 27 August 2018 (right panels). The upper panels demonstrate the westward electrojet dynamics, estimated at the $22.06^{\circ}$ geographic longitude ( $112^{\circ}$ CGMLon). In Fig. 3 the magnetic field X-component at the selected INTERMAGNET stations during the considered substorms (upper panels) and SML indexs, By, Bz, and Vx are presented. The stations CGMLat is indicated to the right. In the figures, the stations are arranged by their magnetic latitude locations. The substorm onsets are indicated by the red vertical lines (determined by PEL, OUJ, OUJ stations data, respectively). 


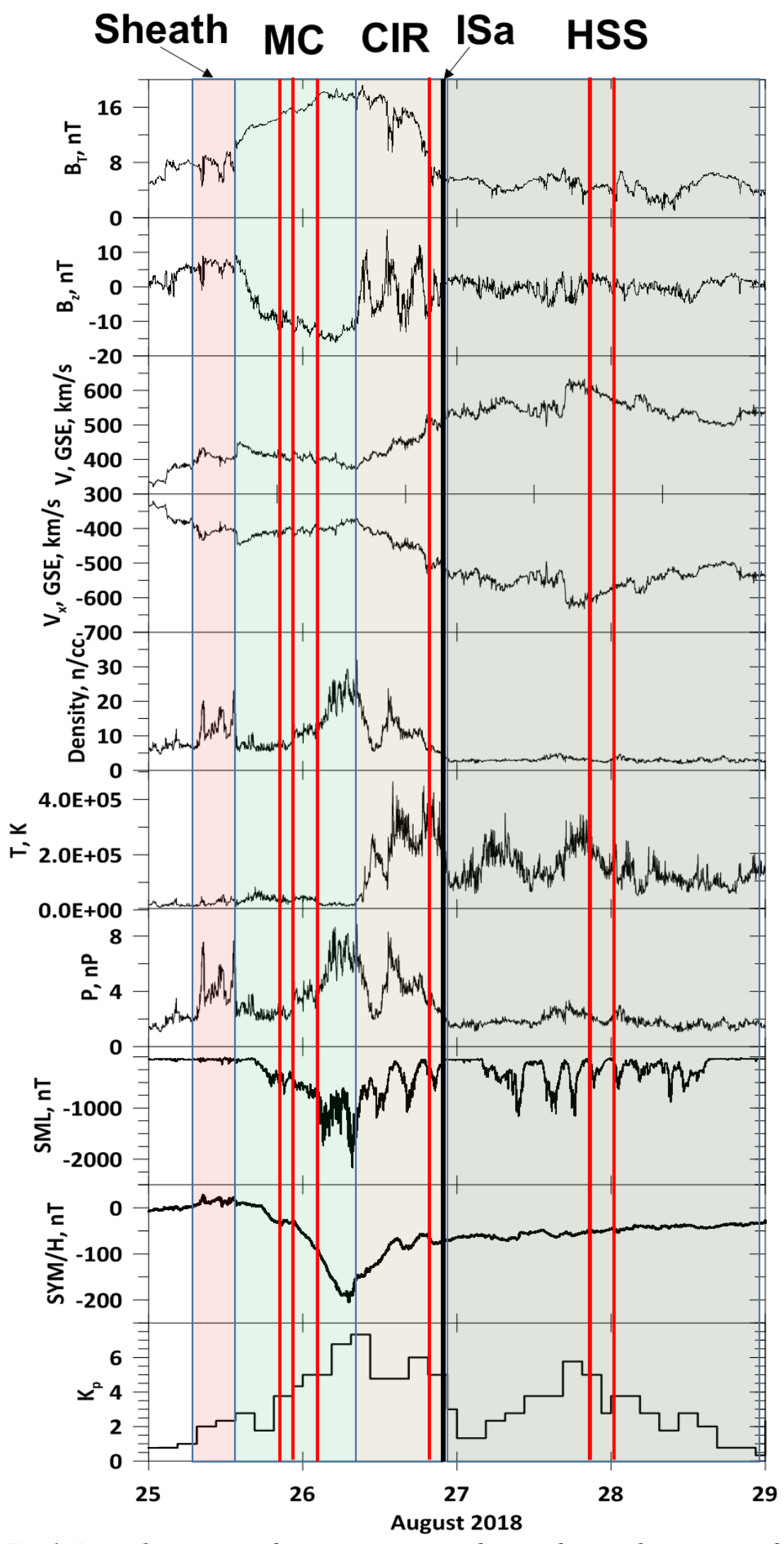

Fig.1. Interplanetary and geomagnetic conditions during the storm on 25 August 2018..The structures in the solar wind are marked by rectangles in different colours and inscribed in the upper part of the figure. The moment of interplanetary shock (IS) arrival is indicated by straight vertical line. The time of the observed substorms are marked by red vertical lines. 


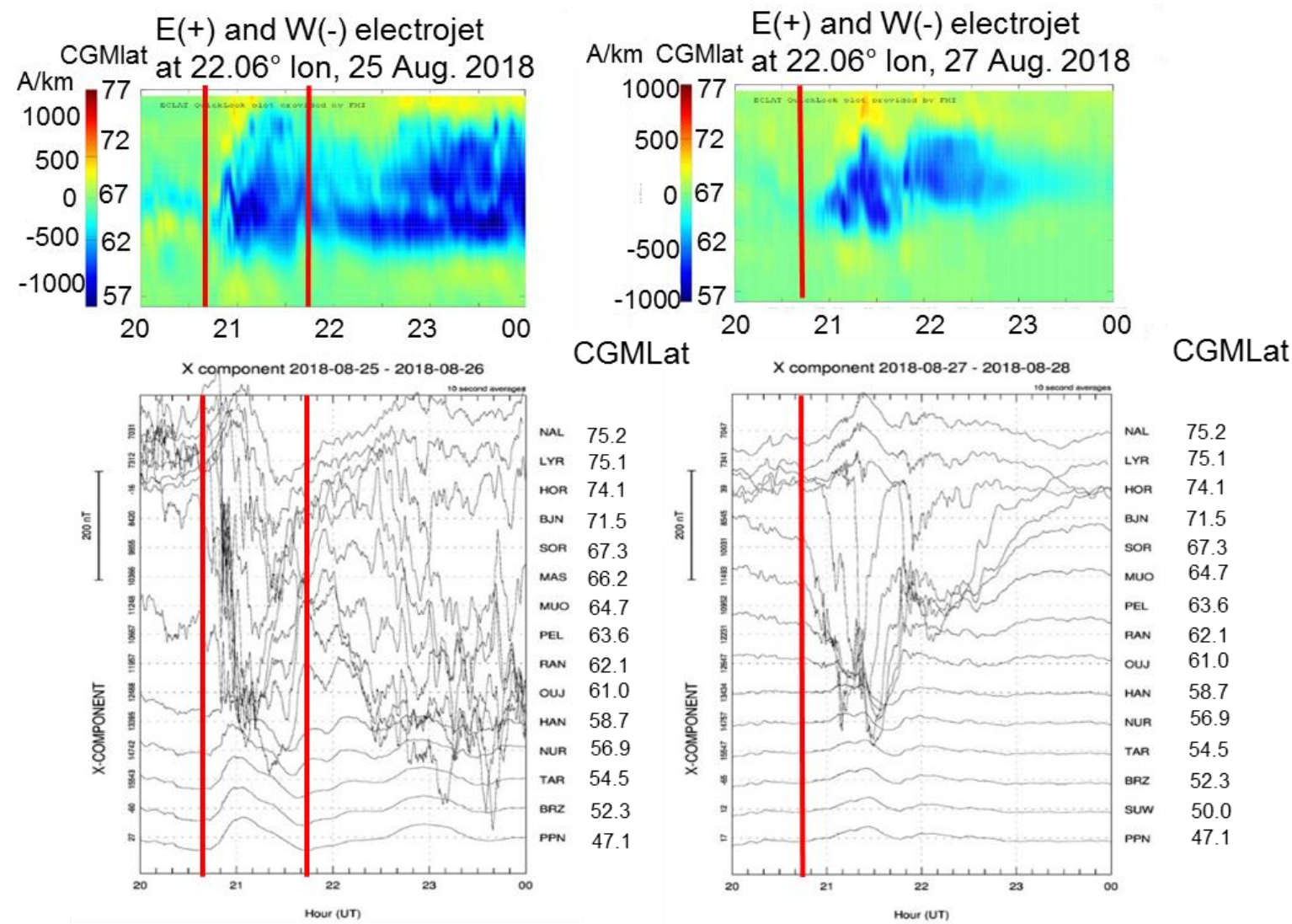

Fig.2. Ionospheric equivalent currents (up), and $B_{x}$ data from IMAGE latitudinal chain for the substorms (down). The substorms onset times are marked by red vertical lines. The left panels show the substorms on 25 August 2018, during the main storm phase, and the right panels - the substorm on 27 August 2018, in the late recovery phase.

\section{Substorm at 20:40 UT on 25 August 2018}

This substorm developed in the beginning of the the main storm phase, at the time of the magnetic cloud (MC) in the solar wind (see Fig. 1). At the substorm onset, SYM/H was $-38 \mathrm{nT}$. The minimal value of SML index during the substorm was $-721 \mathrm{nT}$. The westward electrojet moved fast to the Nord, and surpassed $77^{\circ}$ CGMLat. A movement to the South was also observed (Fig.2, left upper panel). The substorm onset was at $\sim 64^{\circ}-65^{\circ}$ CGMLat (PEL-MUO stations). The disturbances in the X-component are clearly expressed to the North, to NAL (75.25 ${ }^{\circ}$ CGMLat) as well as to the South, to OUJ (61.0 CGMlat) (Fig. 2, left bottom panel). At the lower latitudes, a positive bay in the X-component was observed at all stations to the South from HAN (58. $7^{\circ} \mathrm{CGMlat)} \mathrm{(Fig.} \mathrm{2,} \mathrm{left} \mathrm{bottom} \mathrm{panel,} \mathrm{and} \mathrm{Fig.} \mathrm{3,} \mathrm{left} \mathrm{upper} \mathrm{panel).} \mathrm{The} \mathrm{positive}$ bay lasted about 1 hour.

\section{Substorm at 21:45 UT on 25 August 2018}

The second substorm originated just after the first one, also in the beginning of the main phase of the storm, during MC (Fig.1). At the onset, SYM/H was -33 nT. The minimal SML value was $-646 \mathrm{nT}$. The westward electrojet reached $73^{\circ}-74^{\circ}$ to the North and $\sim 61^{\circ}$ to the South (Fig.2, left upper panel). The strong disturbances in the X-component begun at $61^{\circ}-64^{\circ} \mathrm{CGMLat}$ (OUJ, SOD, LYC stations), reached LYR (75.1 ${ }^{\circ}$ CGMlat) to the North and OUJ $\left(61.0^{\circ}\right.$ CGMlat) to the South (Fig. 2, left bottom panel). A positive magnetic bay was registered at NUR (56.9 ${ }^{\circ}$ CGMlat) and at all stations to the South from NUR (Fig. 2, left bottom panel, and Fig. 3, left upper panel). The positive bay lasted about 1 hour 45 min. 

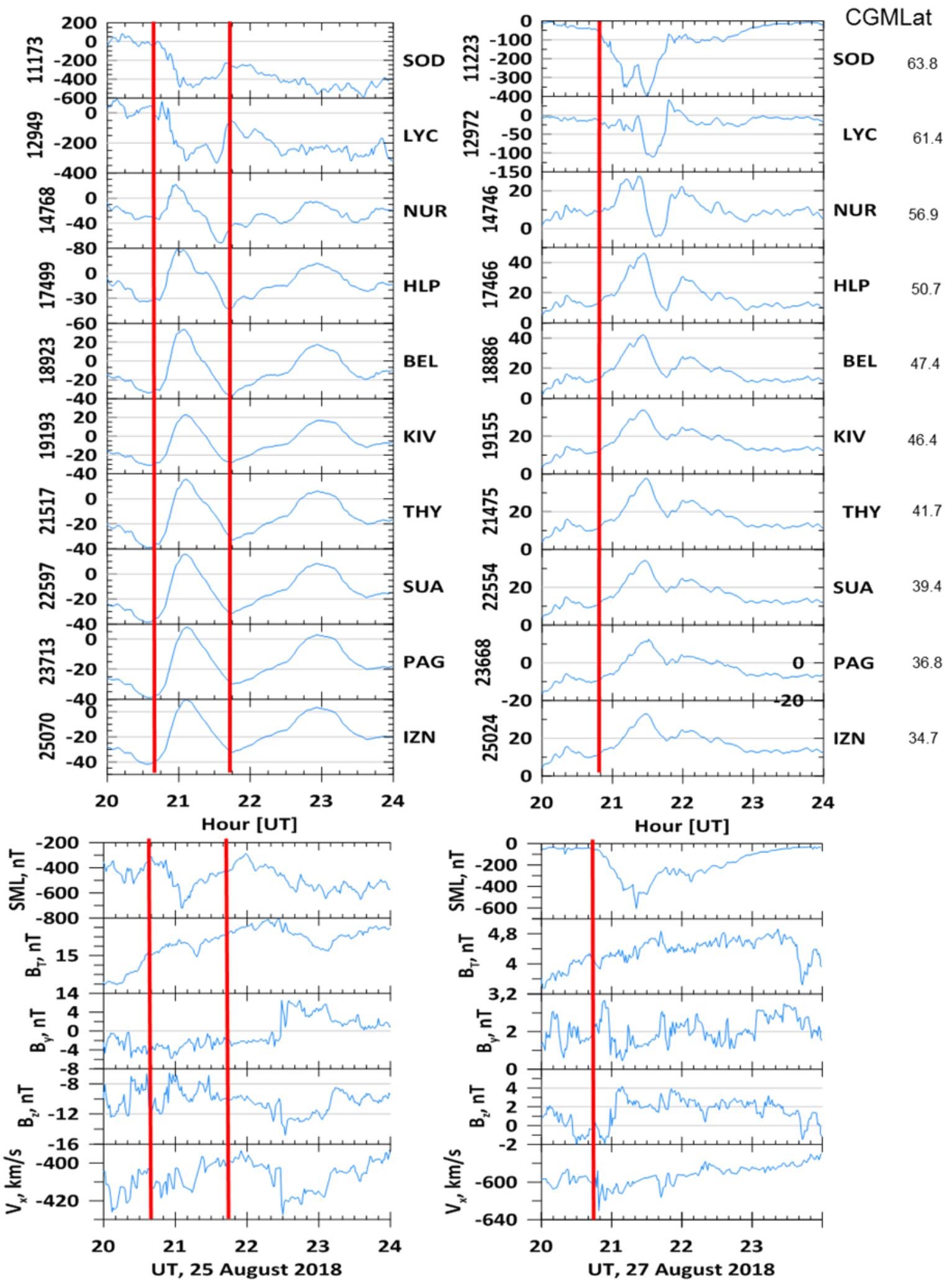

Fig.3. $B_{x}$ data from INTERMAGNET latitudinal chain for the examined substorms (up), and SML index and some characteristics of the SW and IMF during the same time (down).

\section{Substorm at 20:45 UT on 27 August 2018}

The third substorm developed during the late recovery phase of the storm, in the time of a HSS in the solar wind (Fig.1). At the onset, SYM/H was $-51 \mathrm{nT}$. The minimal SML value was $-607 \mathrm{nT}$. The westward electrojet reached $72^{\circ}-73^{\circ}$ to the North and $\sim 63^{\circ}$ to the South (Fig.2, 
right upper panel). The strong disturbances in the X-component begun in the range $62^{\circ}$ $67^{\circ} \mathrm{CGMLat}$ (RAN-SOR stations), reached BJN (71.5 ${ }^{\circ}$ CGMlat) to the North and OUJ $\left(61.0^{\circ}\right.$ CGMlat) to the South (Fig. 2, right bottom panel). A positive magnetic bay was registered at NUR (56.9 ${ }^{\circ}$ CGMlat) and at all stations to the South from NUR (Fig. 2, right bottom panel, and Fig. 3, right upper panel). The positive bay lasted about 1 hour.

\section{Conclusions}

The magnetic bay sign changed from negative to positive between $59^{\circ}$ and $61^{\circ}$ CGMlat (between HAN and OUJ stations) during the examined substorms. This boundary is higher than the observed in a previous study: $50^{\circ}-56^{\circ}$ CGMLat [Guineva et al., 2019]. Hence, this boundary depends on the stage of interplanetary and geomagnetic disturbance. The considered in this work cases happened in the beginning of the main phase and in the late recovery phase of the storm, and SYM/H was only several tens nT, while the previously studied events occurred near the maximal storm development or at a shock arrival, or SYM/H was below $-150 \mathrm{nT}$.

\section{References}

Akasofu S.-I. (1964). The development of the auroral substorm. Planetary and Space Science, 12(4), $273-282$. https://doi.org/10.1016/0032-0633(64)90151-5

Akasofu S.-I., S.Chapman, C.-I. Meng (1965). The polar electrojet. Journal of Atmospheric and Terrestrial Physics, 27(11-12), 1275-1305. https://doi.org/10.1016/0021-9169(65)90087-5

Akasofu, S.-I., \& Meng, C.I.: 1969, A study of polar magnetic substorms, J. Geophys. Res., 74(1), $293-313$. https://doi.org/10.1029/JA074i001p00293

Clausen L. B. N., S.E. Milan, J. B. H. Baker, J. M. Ruohoniemi, K.-H. Glassmeier, J. C. Coxon, B. J. Anderson (2013). On the influence of open magnetic flux on substorm intensity: Ground- and space-based observations. Journal of Geophysical Research. 118, 2958- 2969, doi:10.1002/jgra.50308.

Despirak I. V., A. A. Lyubchich, H. K. Biernat, A.G. Yakhnin (2008). Poleward expansion of the westward electrojet depending on the solar wind and IMF parameters. Geomagnetism and Aeronomy. 48(3), 284-292.

Despirak I. V., A. A. Lyubchich, N. G. Kleimenova (2014). Polar and High Latitude Substorms and Solar Wind Conditions. Geomagnetism and Aeronomy. 54(5), 619-626.

Feldstein Y. L., G.V. Starkov (1967). Dynamics of auroral belt and geomagnetic disturbances. Planetary and Space Science. 15(2), 209-229.

Guineva V.H., Despirak I.V., Kleimenova N.G. (2019). Substorms manifestation at high and mid-latitudes during two large magnetic storms, Aerospace Res. in Bulgaria, 31, 27-39, https://doi.org/10.3897/arb.v31.e03, http://journal.space.bas.bg/arhiv/n\%2031/Articles/3 Guineva.pdf

Kisabeth J. L., G. Rostoker (1974). The expansive phase of magnetospheric substorms: 1. Development of the auroral electrojets and auroral arcs configuration during substorm. Journal of Geophysical Research. 79(7), 972984.

McPherron R.L., C.T. Russell, M.P. Aubry, (1973). Satellite studies of magnetospheric substorms on August 15, 1968. 9. Phenomenological model for substorms. J. Geophys. Res. 78, 3131

McPherron, R. L., X. Chu (2017). The mid-latitude positive bay and the MPB index of substorm activity. Space Science Reviews, 206(1-4), 91-122. https://doi.org/10.1007/s11214-016-0316-6

McPherron, R. L., X. Chu (2018). The midlatitude positive bay index and the statistics of substorm occurrences. Jurnal of Geophysical Research: Space Physics, 123, 2831-2850. https://doi.org/10.1002/2017JA024766

Meng, C.I., Akasofu, S.-I.: 1969, A study of polar magnetic substorms .2. 3-dimensional current system, J. Geophys. Res., 74(16), 4035-4053. https://doi.org/10.1029/JA074i016p04035

Tanskanen E. I., T. I. Pulkkinen, A. Viljanen, K. Mursula, N. Partamies, J. A. Slavin (2011). From space weather toward space climate time scales: Substorm analysis from 1993 to 2008. Journal of Geophysical Research. 116, A00I34, doi:10.1029/2010JA015788.

Wang H., H. Lühr, S. Y. Ma, P. Ritter (2005). Statistical study of the substorm onset: its dependence on solar wind parameters and solar illumination. Annales Geophysicae. 23, 2069-2079. doi:10.5194/angeo-23-2069-2005.

Yermolaev, Yu.I., Nikolaeva, N.S., Lodkina, I.G., Yermolaev, M.Yu.: (2009). Catalog of large-scale solar wind phenomena during 1976-2000. Cosmic Research (Engl. Transl.), 47, 81-94. 


\title{
Strong High-Latitude Geomagnetic Response to the Initial Phase of the Magnetic Storm on 5 April 2010
}

\author{
Kleimenova N.G. ${ }^{1}$, Gromova L.I. ${ }^{2}$, Gromov S.V. ${ }^{2}$, Malysheva L.M. ${ }^{1}$ \\ ${ }^{1}$ Schmidt Institute Physics of the Earth RAS, Moscow, Russia \\ ${ }^{2}$ IZMIRAN, Moscow, Russia \\ e-mail:kleimen@ifz.ru
}

\begin{abstract}
The global high-latitude geomagnetic signature of the first magnetic storm (5-6 April 2010) of the very beginning of the solar cycle 24 has been studied using the ground-based magnetometer networks (SuperMAG, IMAGE and INTERMAGNET) data as well as the data of the ionospheric magnetometer satellite system AMPERE. Non-typical geomagnetic response of this storm was found: the strongest geomagnetic disturbances have been observed in the storm initial phase, but not in the storm main phase. The high-latitude dayside magnetic effects as the occurrence of the negative magnetic bays at polar latitudes and their association with a very strong night-side substorm are discussed. It was shown that this geomagnetic feature was caused by the peculiar properties of the solar wind and Interplanetary Magnetic Field (IMF) disturbances in the sheath ahead of the magnetic cloud.
\end{abstract}

\section{Introduction}

The fast-moving ( $V s w$ about $\sim 800 \mathrm{~km} / \mathrm{s}$ ) coronal mass ejection (CME) preceded by an interplanetary shock (SHEATH) arrived at the Earth at 08:26 UT of April 5, 2010 [Möstl et al., 2010; Liu et al. 2011], and was identified as SC of a moderate magnetic storm (Dst min $_{\min }=-81 \mathrm{nT}$ on 6 April). It was the first notable magnetic storm in the very beginning of the 24-th solar cycle, i.e. during the solar minimum, therefore different separate geophysical aspects of this event were widely discussed by many authors, e.g. [Kinrade et al., 2012; McComas et al., 2012; Shimeis et al., 2012; Kleimenova et al., 2013; Fathy et al., 2014; Lu et al., 2014; Baishev et al., 2015; Victor et al., 2016; Nishimura et al., 2020]. However, the high-latitude geomagnetic disturbances in the polar region of the dayside magnetosphere remained unexplored, despite these regions are the first magnetosheath areas where the solar wind impacts the magnetosphere. The aim of this paper is to study the dayside high-latitude geomagnetic disturbances associated with this magnetic storm and to show the global feature of the storm geomagnetic response. In our study we used the data from the globally distributed ground-based magnetometer networks: SuperMAG (http://supermag.jhuapl.edu/), IMAGE (http://space.fmi.fi/image/) and INTERMAGNET (http://www.intermagnet.org/)) as well as the ionospheric magnetometer data from the 66 Iridium Communications satellites AMPERE (http://www.ampere.jhapl.edu/).

\section{Interplanetary conditions}

The solar wind and Interplanetary Magnetic Field variations during the magnetic storm on 5 April 2010 were non-typical (Fig. 1): the main solar wind and IMF irregularities occurred not in the storm main phase, as it is typical, but during the ahead interplanetary shock (SHEATH) under strong solar wind dynamic pressure $(P s w)$ reached $\sim 10 \mathrm{nPa}$ and even more (Fig. 1). The duration of the southward IMF was only about 1 hour, up to 09:25 UT, thus, the significant Dst $(\mathrm{SymH})$ decreasing could not develop. The steady southward IMF occurred only after $\sim 13 \mathrm{UT}$, and the real storm main phase started. Thus, the preceded time interval (up to $\sim 13$ UT) can be attributed to the storm initial phase. After the SC, the estimated location [after Kuznetsov and Suvorova, 1998] of the dayside magnetopause shifted from $\sim 10.5$ Re to $\sim 7.5$ Re. 
a)

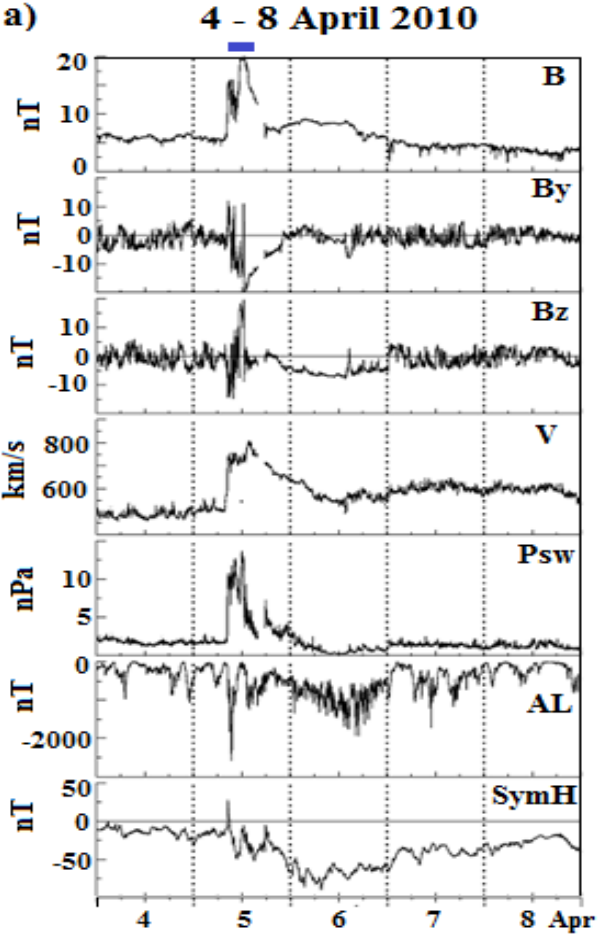

b) 5 April 2010

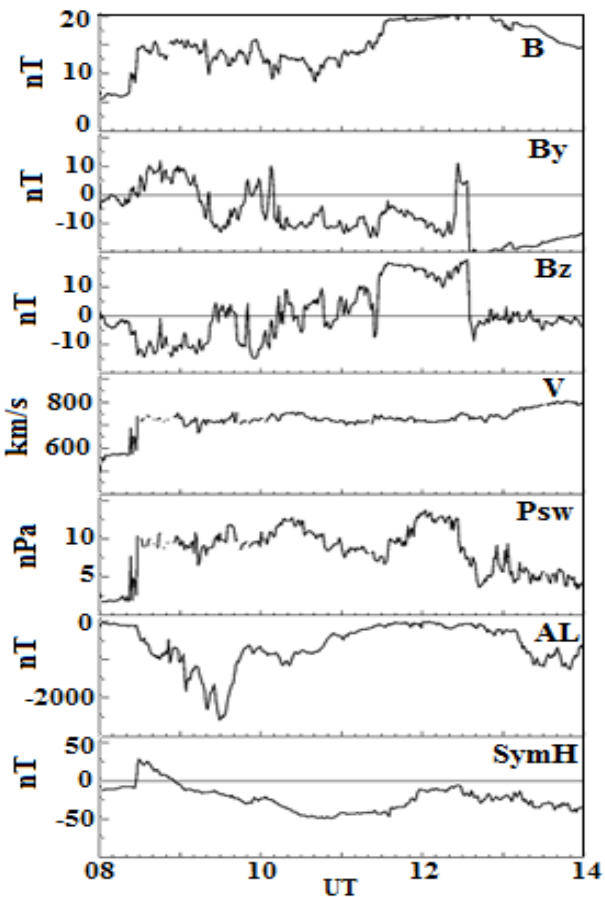

Fig. 1. The interplanetary conditions of the magnetic storm on 5-6 April 2010: a)-during 5 days around this storm, b) in the storm beginning before its main phase.

5 April 2010

a)

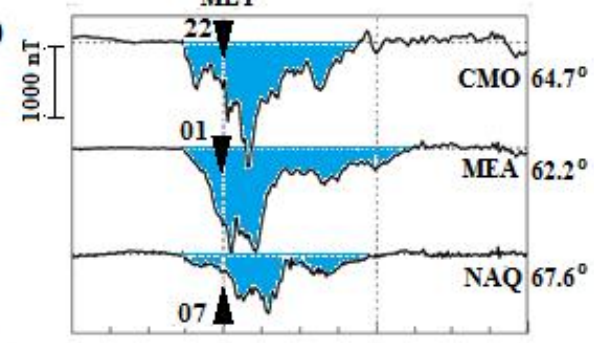

b)

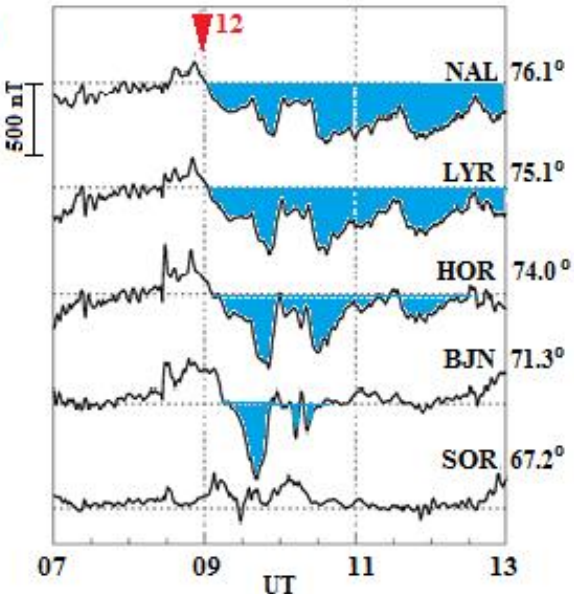

c)
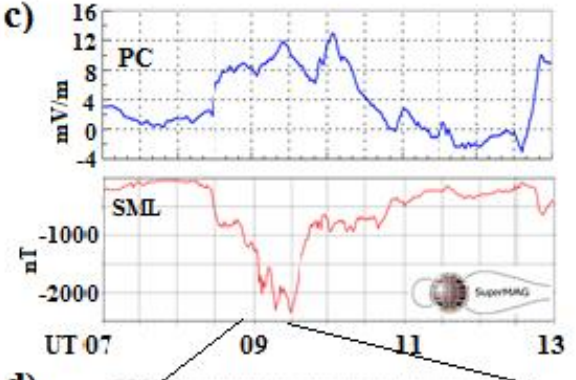

d)

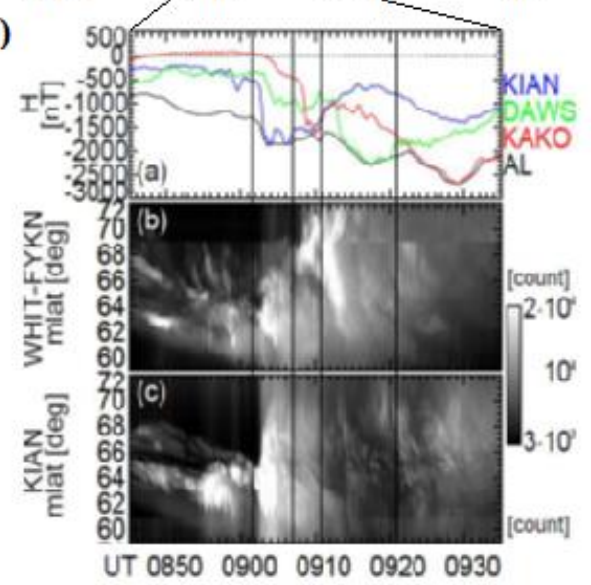

Fig. 2. Magnetograms from selected high-latitude stations: a) night-side, b) dayside; c) the variations of the PC and SML indexes; d) magnetograms from selected THEMIS ground-based stations and auroral keograms demonstrating the substorm growth phase and auroral breakup (adapted from [Nishimura et al., 2020]). 


\section{High-latitude geomagnetic response}

An interplanetary shock with a large southward IMF compressed the Earth's magnetosphere and created the shock aurora [Nishimura et al., 2020]. About 20 min later, the substorm growth phase arc can be seen as a latitudinally thin arc starting at 08:38 UT at $65^{\circ}$ MLAT (Fig. 2d) followed by rapid equatorward motion which was much faster than that for typical substorms. About 20 min later, the large dipolarizations ("overdipolarization") took place at GOES 11 at 09:03 and 09:08 UT in the midnight sector [Nishimura et al., 2020], and also by the three THEMIS spacecraft near $X=-11, Y=-2$ RE [Connors et. al., 2011]. That resulted the bright auroral breakup (Fig. 2d) followed by the very strong substorm (supersubstorm) as it seen in Fig. 2c demonstrated very strong values of the SML index (up to $2500 \mathrm{nT}$ ). This index is similar to the well-known AL-index, but derived from the 88 auroral SuperMAG stations [Gjerloev et al., 2012].

The magnetograms from the selected high-latitude nightside and dayside stations are shown in Fig. 2a, b for the time interval of the storm initial phase. It is seen that simultaneously with the strong nighttime negative magnetic bays, developed after the dipolizations and auroral breakup (Fig. 2d), the bay-like geomagnetic disturbances occurred at the dayside polar latitudes (71-76 ${ }^{\circ}$ MLAT) but with the significant smaller amplitudes (note the different scale of the night and day graphs). Moreover, there were two additional negative magnetic bays at the dayside 74-76 MLAT: at 10:20-11:20 UT (which is not discussed here) and 11:30-12:30 UT, both observed under the northward IMF (IMF Bz>0).

\section{Global feature of the geomagnetic response}

The global distribution of the magnetic vectors measured by the SuperMAG stations is shown in Fig. 3 for three intervals: a) few minutes after the SC, b) near the maximum of the SML-index, and c) in the middle of the last polar negative magnetic bay (Fig. 2b). It is shown (Fig. 3a) that the SC under the southward IMS caused the moderate geomagnetic disturbances on the day and night sides but with the different directions. The ground-based data (Fig. 2) indicate the negative magnetic deviation on the nightside and positive one on the dayside. The same effect is demonstrated by the AMPERE ionospheric satellites (Fig. 4). It is seen (Fig. 4a) that the ionospheric current direction is different in the afternoon (upward field aligned current FAC enhancement) and before noon (downward FAC enhancement).

In the supersubstorm maximum (near 09:30 UT), the strongest ground-based magnetic disturbances according to the SuperMAG data (Fig. 3b) were observed in the evening. They were accompanied by the high-latitude magnetic disturbances on the dayside and in the early morning.

\section{April 2010}

a)

b) c)

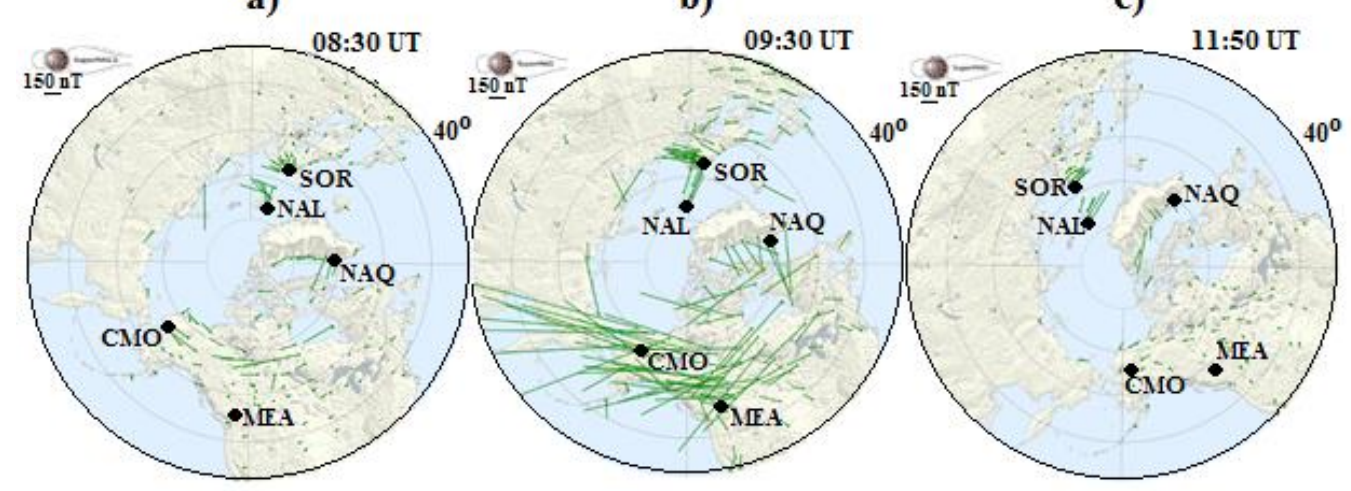

Fig.3. The global SuperMAG maps of the magnetic vectors rotated by $90^{\circ}$ to show the direction of ionospheric equivalent electric currents at selected UT moments 
That was confirmed by the AMPERE ionospheric measurement (Fig. 4b) demonstrated the strong vortex in the evening indicating the very enhanced upward FACs as well as the westward electrojet from the noon to the Harang discontinuity before the midnight.

The negative magnetic bay at 11:30-12:30 UT (Fig. 2b) was observed only at the polar latitudes in the dayside (Scandinavian IMAGE magnetometer chain). There was no notable magnetic activity in the night sector (Fig. 2a) as it is also seen by SuperMAG measurements (Fig. 3c). The ionospheric AMPERE satellite data (Fig. 4c) show the polar-latitudes vortex located near the noon and the correspondent enhancement of a pair of the downward and upward FACs at the dayside polar latitudes. The night sector was very slightly disturbed.

\section{April 2010}

\section{a) $08: 30 \mathrm{UT}$}

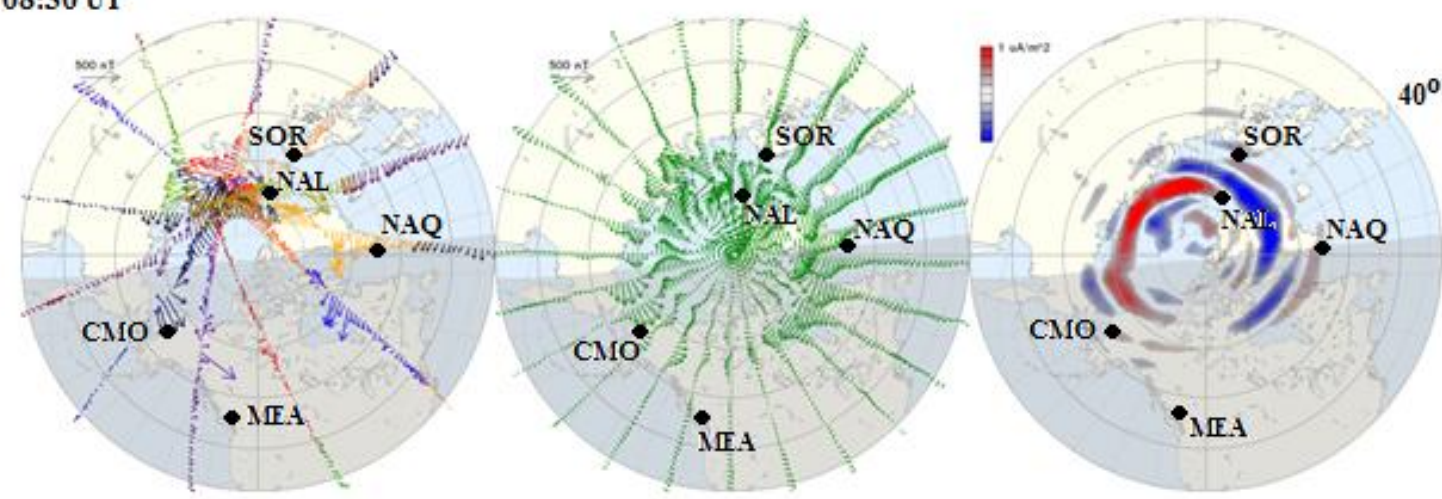

b) $09: 30 \mathrm{UT}$
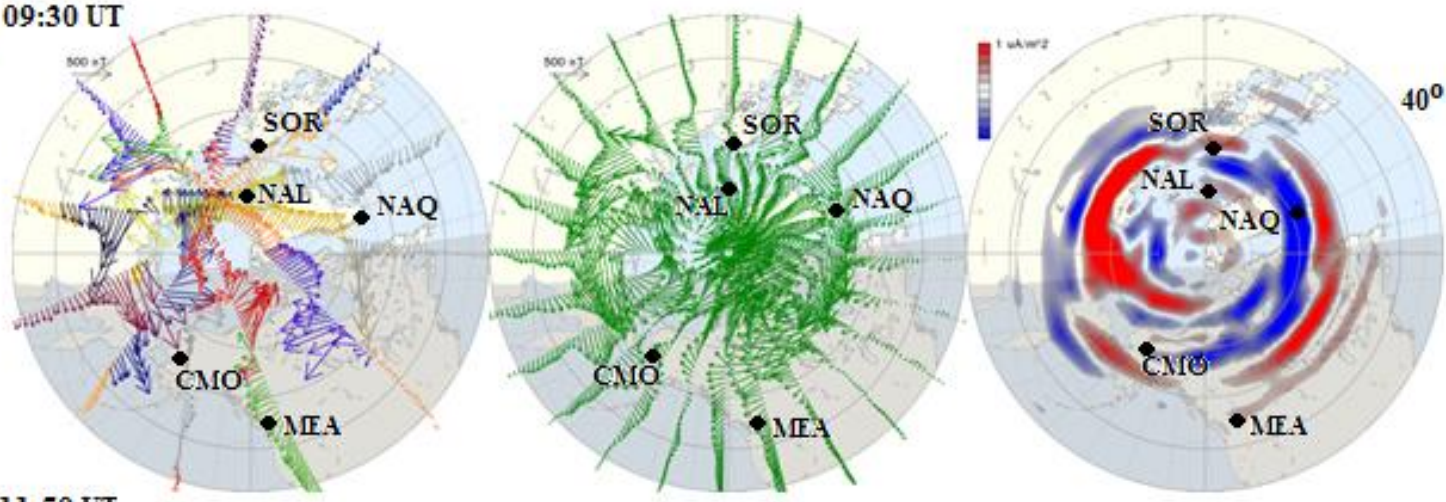

c) $11: 50 \mathrm{UT}$

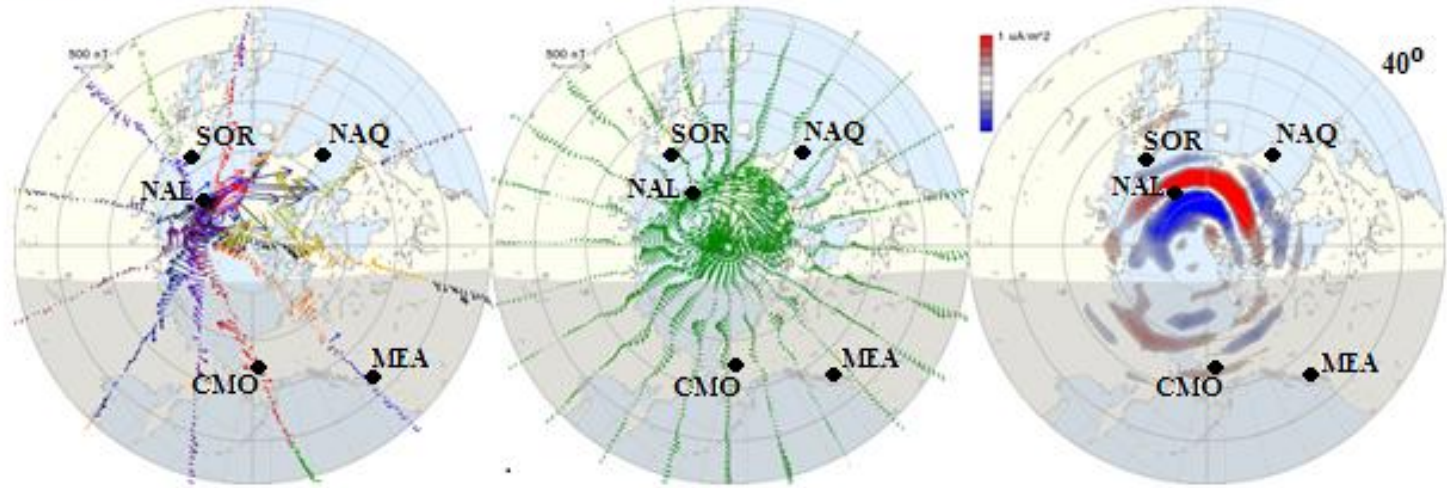

Fig. 4 The AMPERE magnetic field perturbations over the northern polar region (different color vectors represent measurements from the different satellites) and the result of their spherical harmonic analysis as well as calculated distribution of the field aligned currents (FACS) at the selected UT times where the blue marks the downward FAC and the red marks the upward FAC. 


\section{Discussion}

The first notable geomagnetic storm of the solar cycle 24 has happened on 5-6 April 2010. The storm was triggered by the compressed solar wind sheath region preceding a fast-moving CME. Despite being a relatively weak storm (the minimum $D s t$ was $\sim 80 \mathrm{nT}$ on 6 April), it had some devastating space weather impacts, including developing of the strong supesubstorm with the magnitude $\sim 2500 \mathrm{nT}$. This auroral electrodynamics is strongly driven but without the intense ring current injection which is typically associated with major storms. This is an interesting feature of the storm of 5 April 2010.

The strongest geomagnetic perturbations have been observed during the passage of the sheath ahead of the CME. A few minutes after SC under southward IMF and elevated solar wind dynamic pressure, the auroral oval moved equatorward and a supersubstorm started to develop. These processes in the nightside magnetosphere and ionosphere have been detailly analyzed in the paper [Nishimura et al., 2020]. It was shown that the supersubstorm exhibited multi-step enhancements of unusually large electrojets with an extremely large poleward boundary intensification (PBI). Very large vortex in the evening ionosphere and the corresponding enhanced FACs were observed by AMPERE data (Fig. 4). The magnetic disturbances spaced in the longitude to the day side where the negative magnetic bay was observed near the poleward boundary of the auroral oval (Fig. 2). Thus, the supersubstorm was recorded in the global scale.

At 09:25 UT the sign of IMF suddenly changed and the night side magnetic activity and SML index began to decrease, but the magnitude of dayside polar bay increased due to occurred and enhanced NBZ FACs caused by the positive IMF Bz.

The third interval from $\sim 11: 30$ to $\sim 12: 45$ UT shown in the SuperMAG and AMPERE data corresponds to the magnetosphere response of a jump of the strong northward field (IMF- $\mathrm{Bz} \sim$ $+15 \mathrm{nT}$ ) under strong negative IMF By. The negative magnetic bay was observed only in the dayside polar region. The fluctuations of $B z$ and $B y$ IMF components (Fig. 1) lead to conditions where the magnetic reconnection occurs near the cusp, generating intense dayside field-aligned currents (FACs) [Möstl et al., 2010] caused the considered polar magnetic bay.

One of the indicators of the solar wind energy input to the magnetosphere is an increasing of the polar cap PC-index [Stauning, 2013; Troshichev et al., 2014]. The strong enhancement of PC-index before the maximum of supersubstorm (SML-index minimum) is seen in Fig. 2c. The second PC-index remarkable enhancements were observed before the dayside polar negative magnetic bays supporting that their source is associated with the solar wind energy input.

\section{Conclusion}

The strong high-latitude geomagnetic response of the not strong magnetic storm was found. The significant geomagnetic disturbances have been observed in the storm initial phase during the passage of the SHEATH ahead a CME. The large supersubstorm occurred accompanying by the dayside negative magnetic bay observed near the poleward boundary of the auroral oval. The similar bays were also recorded at the cusp latitudes under the northward IMF appearance.

It was shown that this geomagnetic feature was caused by the peculiar properties of the solar wind and IMF irregularities in the SHEATH region ahead of the magnetic cloud that triggered this magnetic storm.

\section{References}

Anderson, B.J., Korth, H., Waters, C.L., Green, D.L, Merkin, V.G, Barnes, R.J., and Dyrud, L.P. (2014). Development of large-scale Birkeland currents determined from the Active Magnetosphere and Planetary Electrodynamics Response Experiment, Geophys. Res. Lett., 41(9), 3017-3025. https://doi.org/10.1002/2014GL059941

Baishev, D.G., Moiseyev, A.V., Boroyev, R.N., Kobyakova, S.E., Stepanov, A.E., Mandrikova, O.V., Solovev, I.S., Khomutov, S.Yu., Polozov, Yu.A., Yoshikawa, A., and Yumoto, K. (2015). Magnetic and ionospheric observations in the far eastern region of Russia during the magnetic storm of 5 April 2010, Sun and Geosphere, $10(2), 133-140$. 
Connors, M., Russell, C.T., and Angelopoulos, V. (2011). Magnetic flux transfer in the 5 April 2010 Galaxy 15 substorm: an unprecedented observation, Ann. Geophys., 29, 619-622. https://doi.org/ 10.5194/angeo-29-6192011

Fathy, I., Amory-Mazaudier, C., Fathy, A., Mahrous, A.M., Yumoto, K., and Ghamry, E. (2014). Ionospheric disturbance dynamo associated to a coronal hole: Case study of 5-10 April 2010, J. Geophys. Res. Space Physics, 119, 4120-4133. https://doi.org/10.1002/2013JA019510

Gjerloev, J.W. The SuperMAG data processing technique (2012). J. Geophys. Res., V. 117(A9), A09213. https://doi.org/10.1029/2012JA017683

Kinrade, J., Mitchell, C.N., Yin, P., Smith, N., Jarvis, M.J., Maxfield, D.J., Rose, M. C., Bust, G.S., and Weatherwax, A.T. (2012). Ionospheric scintillation over Antarctica during the storm of 5-6 April 2010, J. Geophys. Res., 117, A05304. https://doi.org/10.1029/2011JA017073

Kleimenova, N.G., Zelinskii, N.R., Kozyreva, O.V., Malysheva, L.M., Solov'ev, A.A., and Bogoutdinov, Sh.R (2013). Pc3 geomagnetic pulsations at near-equatorial latitudes at the initial phase of the magnetic storm of April 5, 2010, Geomagnetism and Aeronomy, 53 (3), 313-320. https://doi 10.1134/S0016793213030092

Kuznetsov, S.N. and Suvorova, A.V.(1998). An empirical model of the magnetopause for broad ranges of solar wind pressure and Bz IMF, in Polar Cap Boundary Phenomena, Moen, J., Egeland, A., and Lockwood, M., Eds., Norwell, MA: Kluwer, 1998, 51-61.

Liu, Y., Luhmann, J. G., Bale, S.D., and Lin, R.P. (2011), Solar source and heliospheric consequences of the 2010 April 3 coronal mass ejection: A comprehensive view, Astrophys. J., 734 (84), https://doi.org/10.1088/0004$\underline{637 X}$

Lu, G., Hagan, M. E., Häusler, K., Doornbos, E., Bruinsma, S., Anderson, B. J., and Korth, H. (2014), Global ionospheric and thermospheric response to the 5 April 2010 geomagnetic storm: An integrated data-model investigation, J. Geophys. Res. Space Physics, 119, 10,358-10,375, https://doi.org/10.1002/2014JA020555.

McComas, D.J., Buzulukova, N., Connors, M.G., Dayeh, M.A., Goldstein, J., Funsten, H.O., Fuselier, S., Schwadron, N.A., and Valek, P. (2012). Two wide-angle imaging neutral-atom spectrometers and interstellar boundary explorer energetic neutral atom imaging of the 5 April 2010 substorm, J. Geophys. Res., 117, A03225, https://doi.org/10.1029/2011JA017273

Möstl, C., Temmer, M., Rollett, T., Farrugia, C.J., Liu, Y., Veronig, A.M., Leitner, M., Galvin, A.B., and Biernat, H.K. STEREO and Wind observations of a fast ICME flank triggering a prolonged geomagnetic storm on 5-7 April 2010 (2010), Geophys. Res. Lett., 37, 24103, https://doi.org/10.1029/2010GL045175

Nishimura, Y., Lyons, L.R., Gabrielse, C., Sivadas, N., Donovan, E.F., Varney, R.H., Angelopoulos, V., Weygand, J.M., Conde, M.G., Zhang, S.R. (2020). Extreme magnetosphere-ionosphere-thermosphere responses to the 5 April 2010 supersubstorm, J. Geophys. Res. https://doi.org/10.1029/2019JA027654

Stauning, P. (2013). The Polar Cap index: A critical review of methods and a new approach. J. Geophys. Res. Space Physics, 118, 5021-5038. https://doi.org/10.1002/jgra.50462

Shimeis, A., Fathy I., Amory-Mazaudier, C., Fleury, R., Mahrous, A.M., Yoshikawa, A., and Groves, K. (2012). Signature of the coronal near the north crest equatorial anomaly over Egypt during the strong geomagnetic storm 5 April 2010, J. Geophys. Res., 117, A07309. https://doi.org/10.1029/2012JA017753

Troshichev, O.A., Podorozhkina, N.A., Sormakov, D.A., and Janzhura, A.S. (2014). PC index as a proxy of the solar wind energy that entered into the magnetosphere: Development of magnetic substorms, J. Geophys. Res.: Space Physics, 119, 6521-6540. https://doi.org/10.1002/2014JA019940

Victor, N.J., Manu, S., Frank-Kamenetsky, A.V., Panneerselvam, C., Anil Kumar, C.P., and Elango, P. (2016), Network of observations on the atmospheric electrical parameters during geomagnetic storm on 5 April 2010 , J. Geophys. Res. Space Physics, 121, 2407-2417. https://doi.org/10.1002/2015JA022080 


\title{
Variations of the lonosphere Scintillation Parameters on the Observations of the Cosmic Radio Sources at the Decameter Wave Range During Two Cycles of the Solar Activity
}

\author{
Lytvynenko O. A., Panishko S. K. \\ Observatory URAN-4, Institute of Radioastronomy NASU \\ E-mail: spanishko@ukr.net
}

\begin{abstract}
.
Effect of the ionosphere scintillations is caused by interaction of ionosphere plasma with radio emission that propagating through ionosphere and it is very essential at decameter radio wave range. Scintillations are reflected the state of irregularity structure of ionosphere in the time and in the certain place and thus they are connected with cosmic weather. Index (intensity), period (characteristic time) and also spectral index (slope of the time spectrum of intensity fluctuations) are used as characteristics of the scintillations. Estimations of the indices, periods and spectral indices of the ionosphere scintillations were obtained on the observations of the power cosmic radio sources on the radio telescope URAN-4 at the frequencies 20 and $25 \mathrm{MHz}$ for two time intervals from 1998 up to 2007 and from 2011 up to 2018. These time intervals are coinciding to a large extent with 23-th and 24-th cycles of the solar activity. This data allowed the studying of the long time variations of the irregularity structure of ionosphere that can be attributed to the category of cosmic climate and as well allow the investigations their connection with other parameters of the cosmic climate. Also the variations of the ionosphere scintillation parameters are considered for several time intervals during two cycles of the solar activity.
\end{abstract}

\section{Introduction}

Investigations of the variations of the cosmic weather components during time intervals comparable to duration of solar cycle allow getting more clear understanding about interconnection of active processes that occurred on the Sun and in the near-earth space. In particular, the variations of parameters of ionosphere scintillations which are part of the cosmic weather reflect the state of the irregularity structure of ionosphere. In early investigations that carried out in the equatorial and polar regions where scintillation effect expressed most strongly it was found that scintillations with large intensity sooner appeared during increasing of solar activity [Aarons J., 1982]. In the region of middle latitudes where scintillations are considered weak the investigations of ionosphere scintillations are not such numerous [Kintner P. M. et al, 2007]. Interaction of the parameters of the midlatitude ionosphere scintillations with the indices of solar and geomagnetic activity expressed not so clearly. Long series of measurements of the parameters of ionosphere scintillations were obtained from observations of the power cosmic radio sources on radio telescope of decameter wave range URAN-4 during two time intervals that coincide to a large extent with periods of two cycles of the solar activity. This data permits researching of long duration variations of irregularity structure of ionosphere that can be attributed to the category of cosmic climate. Further in the works the behavior of parameters of ionosphere scintillations during 23th and 24th cycles of solar activity were analyzed and also the comparison of these parameters with others indices of cosmic climate was fulfilled.

\section{Observations data}

Observations of the power cosmic radio sources - 3C144, 3C274, 3C405, 3C461 - are carry out on the radio telescope URAN-4 at frequencies 20 and $25 \mathrm{MHz}$ in monitoring regime. They have compact components that scintillate on ionosphere irregularities. The most complete series of observations with digital registration of data were obtained during two time intervals and information about them presented in Table 1. In result of the processing of radio source records 
the parameters of ionosphere scintillations were obtained for each record: $S I$ - index; $S T$ characteristic time (period); $S P$ - spectral index. The observation technique and processing method are presented in work [Derevyagin V. G. et al, 2019]. These parameters were compared with indices of solar and geomagnetic activity - with solar flux at radio wave $10.7 \mathrm{~cm} \mathrm{F10} \mathrm{and}$ with planetary $A p$-index.

Table 1. Characteristics of the solar cycles and the time intervals of observations

\begin{tabular}{ccccccc}
\hline $\begin{array}{c}\text { Number } \\
\text { of cycle }\end{array}$ & $\begin{array}{c}\text { Start } \\
\text { date }\end{array}$ & $\begin{array}{c}\text { Finish } \\
\text { date }\end{array}$ & $\begin{array}{c}\text { Date of } \\
\text { maximum }\end{array}$ & $\begin{array}{c}F 10, \\
\text { SFU }\end{array}$ & $\begin{array}{c}\text { Time intervals } \\
\text { of observations }\end{array}$ \\
\hline 23 & 05.1996 & 12.2008 & 11.2001 & 119.8 & 12.1 & $1998-2007$ \\
\hline 24 & 12.2008 & 05.2019 & 04.2014 & 99.6 & 8.0 & $2011-2018$ \\
\hline
\end{tabular}

Also the characteristics of two solar activity cycles are presented in Table 1. Values of solar flux measured in Solar Flux Units (SFU). Proceeding from the mean over the cycle values of solar flux and of index $A p$ you can see that the level of solar and geomagnetic activity much decreases in 24th cycle in comparison with 23th cycle. As can see from Table 1 observation intervals in a large extent coincide with periods of 23th and 24th solar cycles.

\section{Results and their discussion}

Mean values of parameters of ionosphere scintillations calculated over the cycle for 4th radio sources during 23th and 24th solar cycles are showed on Fig. 1. For regulation data for sources placed in dependence of right ascension of radio source. Total form of graphics is similar for both cycles. As peculiarities the following can be noted. Values of scintillation index at two frequencies change in larger limits in 24th cycle in comparison with 23th. At frequency $25 \mathrm{MHz}$ values of index SI decreased in 24th cycle and in case of frequency $20 \mathrm{MHz}$ the situation is not so clear. Values of scintillation period $S T$ increased in 24th cycle for two radio sources that observed on the lower heights $(3 \mathrm{C} 144,3 \mathrm{C} 274)$ and they do not change for radio sources that observed near zenith $(3 \mathrm{C} 405,3 \mathrm{C} 461)$. On the one hand such combination testifies to invariability of mean over the cycle drift speed of irregularities. On the other hand, one can assume that changing for 3C144 and 3C274 was associated with anisometry of ionosphere irregularities which was higher in 24th cycle that can be caused by the lower geomagnetic activity. Values of spectral index $S P$ increased or do not changed for 'low' sources and decreased for 'high' sources.

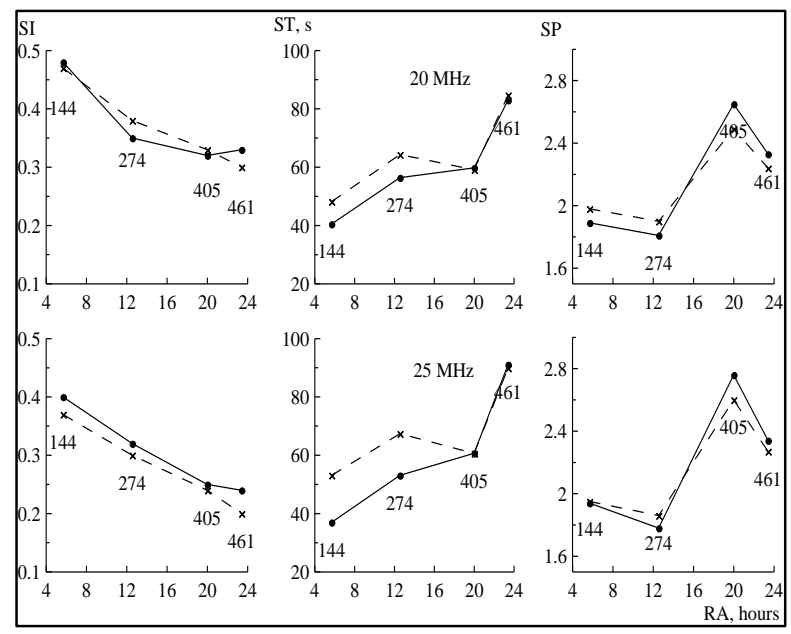

Fig. 1. Mean of the ionosphere scintillation parameters over the cycle for 4 th radio sources during 23th (dots and solid lines) and $24 \mathrm{~h}$ (crests and dash lines) cycles of the solar activity at frequency 20 $\mathrm{MHz}$ (top) and at frequency 25 $\mathrm{MHz}$ (bottom). Abscissa axis right ascension of the radio source

As shown earlier, for example, in work [Panishko S. K., Lytvynenko O. A., 2109] inversely proportional dependence exists between index and period of ionosphere scintillations therefore 
only values of index and spectral index will be considerate in further analysis. Investigating parameters of ionosphere scintillations have notable daily-seasonal dependence [Lytvynenko O. A., Panishko S. K., 2015] consequently the meaning of parameter values over year was used that allowed smoothing the daily-seasonal dependence.

Graphics of yearly mean values index and spectral index of ionosphere scintillations for 4th radio sources at two frequencies during 23th and 24th solar cycles were showed in Fig. 2 and 3. As you can see from figures the graphics of both index and spectral index cannot be interpreted unambiguously. In order to show the behavior of spectral index during 23th cycle of solar activity in work [Panishko S. K., Lytvynenko O. A., 2109] data was transformed in the following way. The polynomials of second order fitted to the yearly mean series of values of indices SI, SP, FIO and Ap for designation of low frequency trend. These trends were normalized to maximum value. Graphics of obtained rows are showed in Fig. 4 and 5 but already for two cycles of solar activity.

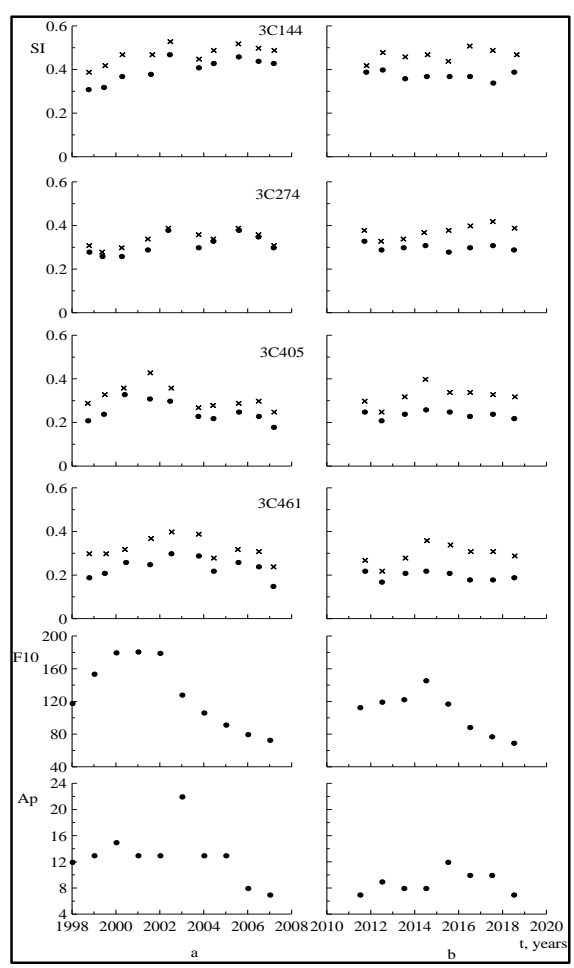

Fig. 2. Yearly mean indices of the ionosphere scintillations for 4 th radio sources: data at frequency $20 \mathrm{MHz}$ marked by crests, at frequency $25 \mathrm{MHz}$ - by dots. Graphics of solar flux on wavelength $10.7 \mathrm{~cm}$ (in SFU) and geomagnetic Ap-index are nrosontod on tho hottom $a-$ data

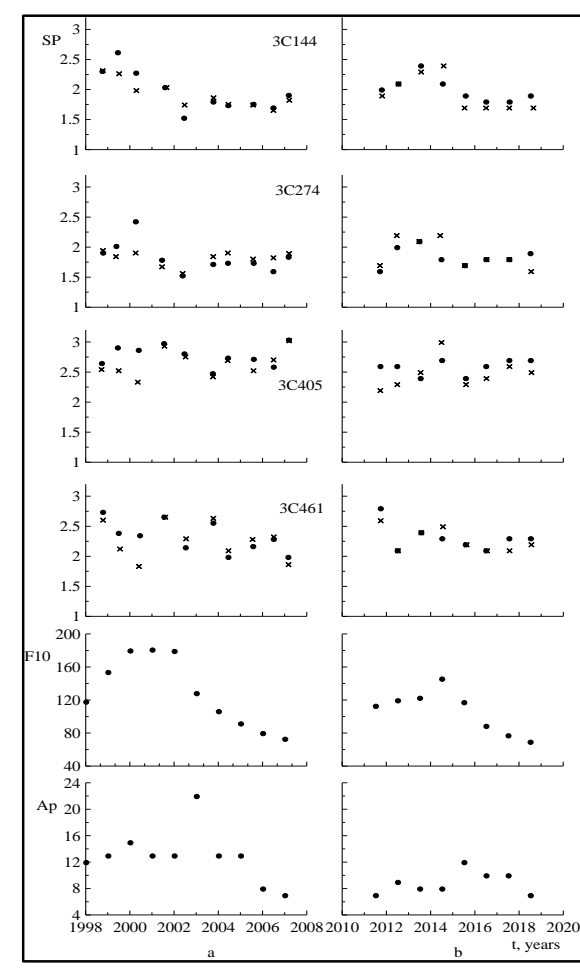

Fig. 3. Yearly mean spectral indices of the ionosphere scintillations for 4th radio sources: data at frequency $20 \mathrm{MHz}$ marked by crests, at frequency $25 \mathrm{MHz}-$ by dots. Graphics of solar flux on wavelength $10.7 \mathrm{~cm}$ (in SFU) and geomagnetic Ap-index are nrosented on the hottom $a$-data

In general, the trends of scintillation index during 23th cycle follow to trends of the indices of solar and geomagnetic activity with the exception of that the curves for two 'low' radio sources descend slower on the falling part of the cycle. During 24th cycle such behavior continues in the case of two 'high' radio sources. The rest data sooner is characterizes by the small correlation or weak anti-correlation. The values of spectral index change less than values of scintillation index during both cycles. The distinction in the behavior of this parameter is that 
the notable anti-correlation changes to the small correlation with the trends of solar radio flux and geomagnetic index.

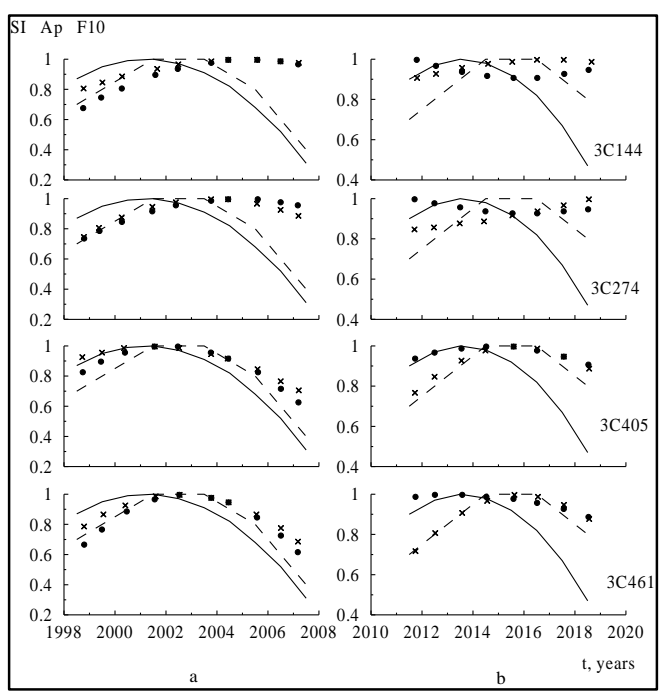

Fig. 4. Trends of the yearly mean values of index of scintillations, solar flux F10 (solid lines) and Ap-index (dash lines). Data at frequency $20 \mathrm{MHz}$ marked by crests, at frequency $25 \mathrm{MHz}-$ by dots; $a$ - data for 23th, $b-$ for

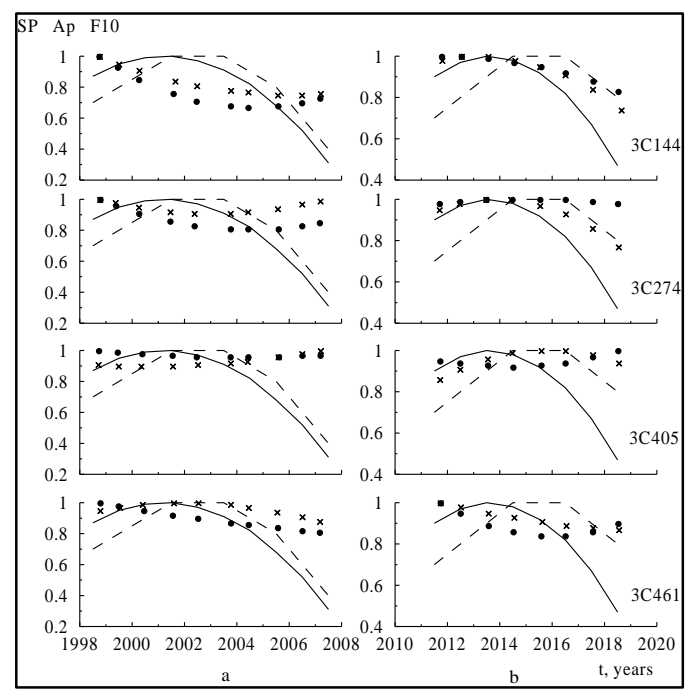

Fig. 5. Trends of the yearly mean values of spectral indices of scintillations, solar flux F10 (solid lines) and Apindex (dash lines). Data at frequency $20 \mathrm{MHz}$ marked by crests, at frequency $25 \mathrm{MHz}-$ by dots; $a$-data for 23 th, $b$-for 24 th

\section{Conclusions}

The analysis of behavior of the yearly mean values of index and spectral indices of ionosphere scintillations on observations obtained on the radio telescope URAN-4 of power cosmic radio sources - 3C144, 3C274, 3C405, 3C461, at the frequencies 20 and $25 \mathrm{MHz}$ during 23th and 24th solar cycles was fulfilled. The distinction is observes in the behavior of the ionosphere scintillation characteristics during the investigating cycles of solar activity. Scintillation index sooner follows to trends of indices of solar and geomagnetic activity during 23th cycle and scintillation index values have weak anti-correlation with them in 24th cycle. Spectral index is more characterizes by the small anti-correlation in 23th cycle and by the weak correlation in the next. This data agrees with others investigations which carried out in the region of the midlatitude ionosphere [Aarons J., 1982]. The preliminary conclusion was made about the increasing of the anisometry of ionosphere irregularities in 24th solar cycle.

\section{References}

Aarons, J. (1982) Global morphology of ionospheric scintillations. Proc. IEEE, 70, 360-378.

Kintner P. M., Ledvina B. M., de Paula E. R. (2007) GPS and ionospheric scintillations. Space Weather, 5, S09003, doi:10.1029/2006SW000260.

Derevyagin V. G., Kravetz R. O., Lytvynenko O. A., Panishko S. K. (2019) Regular observations of the power cosmic radio sources on the radio telescope URAN-4. Processing method, results keeping system and their applying in ionosphere investigations. Proc. of Eleventh Workshop, Primorsko, Bulgaria, June 3-7, 84-88, DOI: 10.31401/WS.2019.proc.

Panishko S. K., Lytvynenko O. A. (2109) Peculiarities in the ionospheric scintillation spectral index behavior by observations of the cosmic radio sources at the decameter wavelengths. Radiofizika i radioastronomia, 24, No $1,44-54$.

Lytvynenko O. A., Panishko S. K. (2015) Seasonal variations of the ionosphere scintillations parameters obtained from the long observations of the power cosmic radio sources at the decameter wave range. Odessa Astronomical Publications, 28, 235-237. 


\title{
Using of Parallel Computing on Gpus for MHD Modeling of Solar Flare Behavior in Real Time Scale
}

\author{
Borisenko A.V. ${ }^{1}$, Podgorny I.M. ${ }^{2}$, Podgorny A.I ${ }^{1}$ \\ ${ }^{1}$ Lebedev Physical Institute of the RAS, Moscow, Russia \\ ${ }^{2}$ Institute of Astronomy of the RAS, Moscow, Russia \\ E-mail: sunw77@mail.ru
}

\begin{abstract}
.
The forecast of solar flares and the appearance of solar cosmic rays (SCR) and other solar flare manifestations is an important scientific and technical task. Magnetohydrodynamic (MHD) modeling in corona above the active region allows us to find the appearance of current sheets in the solar atmosphere which are sources of magnetic energy of flares. In the conditions of complicated configuration of magnetic field in the solar corona the appeared current sheets can be found using specially developed graphical system of search basing of the founding of local current density maximums. MHD simulation in strongly reduced time scale showed the appearance of numerical instability near the photospheric boundary due to unnaturally fast magnetic field increase on the photosphere. To avoid the large number of current density maximums caused by instability near the photospheric boundary, it is necessary to perform MHD simulation in corona in the real scale of time. MHD simulation in real time scale on the ordinary computer took several years. The results of MHD simulation in real scale of time obtained using parallel computing on Nvidia Tesla GPUs showed acceleration of calculations more 100x speed up. The time of calculation of was strongly reduced due to the correct choice of computing equipment and software and using the capabilities of the selected equipment for parallelizing the calculation. The first results of the GPU MHD simulation of flare situation in real time scale for the AR 10365 showed the appearance of current density maximums with Xtype configurations of magnetic field and with a plasma flow which cause to the formation of a current sheet. The simulation in real time scale confirmed the solar flare mechanism based on release of energy accumulated in magnetic field of current sheet.
\end{abstract}

\section{Introduction. The need to apply parallel computations for MHD simulation in the solar corona.}

In view of the appearance of solar flares high in the corona, as well as the impossibility of obtaining from observations the configuration of the magnetic field in the corona, in order to study the mechanism of flares and their prognosis, MHD simulation in the corona above the active region (AR) is necessary [Podgorny et al., 2020]. Flares appear as a result of the release of magnetic energy accumulated in the field of a current sheet, which is formed in the vicinity of a X-type singular line of magnetic field [Podgorny and Podgorny, 2012]. For the magnetic field found as a result of MHD simulation, in a complex configuration above the AR, the position of the current sheet can be determined only with the help of a specially developed graphical search system [Podgorny et al., 2017; Podgorny et al., 2018]. The search system is based on determining the positions of local maxima of the current density, in which the configuration of the magnetic field and plasma flow is analyzed. MHD simulation on the usual computer (Intel (R) Core (TM) i7 CPU 920 @ 2.67GHz) was only carried out in a $10^{4}$ times reduced time scale. Due to the appearance of instability at the photospheric boundary, caused by an unnaturally rapid change in the magnetic field, a large number of fictitious current density

\footnotetext{
81 Topic: Data Processing and Modeling
} 
peaks appeared, masking the current sheets, which made it difficult to use the graphical search system. To get rid of a large number of current density maxima masking current sheets, as well as to establish the evolution of processes in time, it is necessary to perform MHD simulation in real time.

Calculations have shown that using a specially developed difference scheme [Podgorny and Podgorny 2008, 2004; Podgorny et al., 2020], MHD simulation in real time, requires that the time step does not exceed $4 \times 10^{-7}$ days in the main part of the calculated interval. Such simulation cannot be carried out on the usual computer - it would require 8 years of calculation. It becomes necessary to use a 'home supercomputer' with many computational threads, for parallelizing the numerical solution of the used finite-difference scheme.

\section{Selection of computing equipment and software and the use of their capabilities to optimize parallelization of computations}

To use parallel computations, the optimal parameters of the finite-difference scheme were selected and a number of optimizations of the parallel computation algorithm were carried out, primarily related to the exchange of values specified in the computational domain between the main memory of the computer and the memory of the graphics card (GPU), [Podgorny et al., 2020].

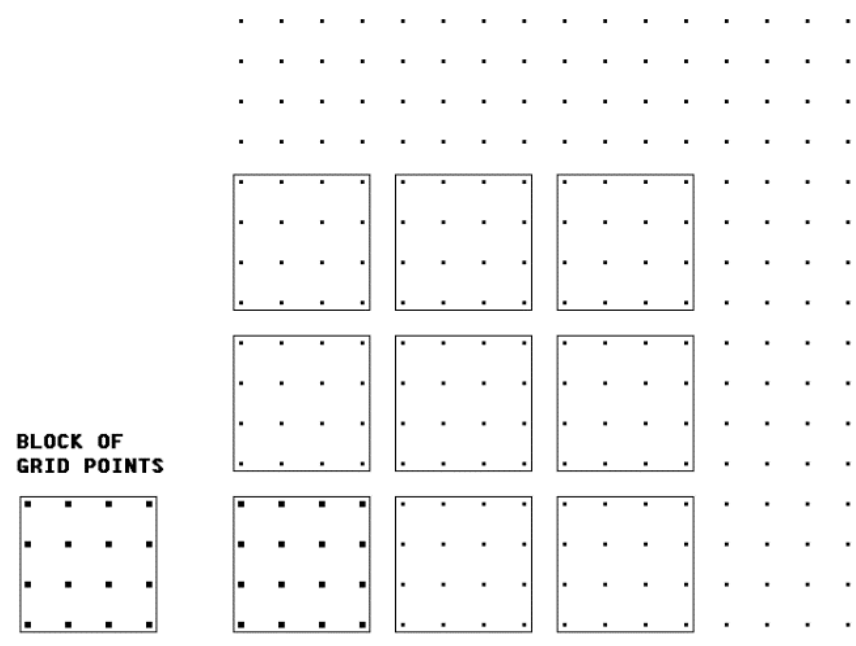

Fig. 1. Coverage of a two-dimensional grid with two-dimensional blocks of points, each of which corresponds to a computational thread.

Parallel computations were carried out using CUDA technology on graphic cards, the computational threads of which carry out parallel computations. The problem was set on 'home supercomputers' rented in the clouds with modern graphics cards. For the calculation, we chose graphic cards with a high computation speed for each thread. The installed software provided additional opportunities for optimizing the parallelization algorithm.

To parallelize computations, modern graphics cards (GPU) Titan-V (Volta), V100 (Volta100), P100 (Pascal-100) were used. The use of the modern language Fortran PGI (Portland Group - Fortran, created specifically for parallelization using graphics cards GPU) made it possible to carry out parallel computation at points of a spatial grid in sequentially selected three-dimensional blocks of threads. To calculate the boundary conditions in the planes bounding the computational domain, two-dimensional blocks of threads were used. An example of covering a two-dimensional grid with two-dimensional flow blocks is shown in Fig. 1, covering the 3D mesh with 3D blocks is similar.

In the majority of calculations 3D blocks were choses $\mathrm{NX} \times \mathrm{NY} \times \mathrm{NZ}=4 \times 4 \times 4=64$ points in block (64 parallel calculating threads, each find values it its point). We also took blocks of 
$8 \times 4 \times 4=128,8 \times 8 \times 4=256$ and more, but this did not lead to an increase in the calculation speed (even led to a slight slowdown in the calculation; a very large number of points in a block led to a significant deceleration). So, so far, we have not managed to increase the calculation speed by 1024 or even 512 times, as we hoped, since so many computational threads physically exist on the graphics card. Now the calculation speed has been increased by parallelizing calculations by 120 times. Experts explain the reason in the waste of time for exchanging data between memory registers.

Also, the use of the selected computing equipment and software made it possible to transfer a large number $(\sim 100)$ of parameters to subroutines that carry out parallelization on graphics cards.

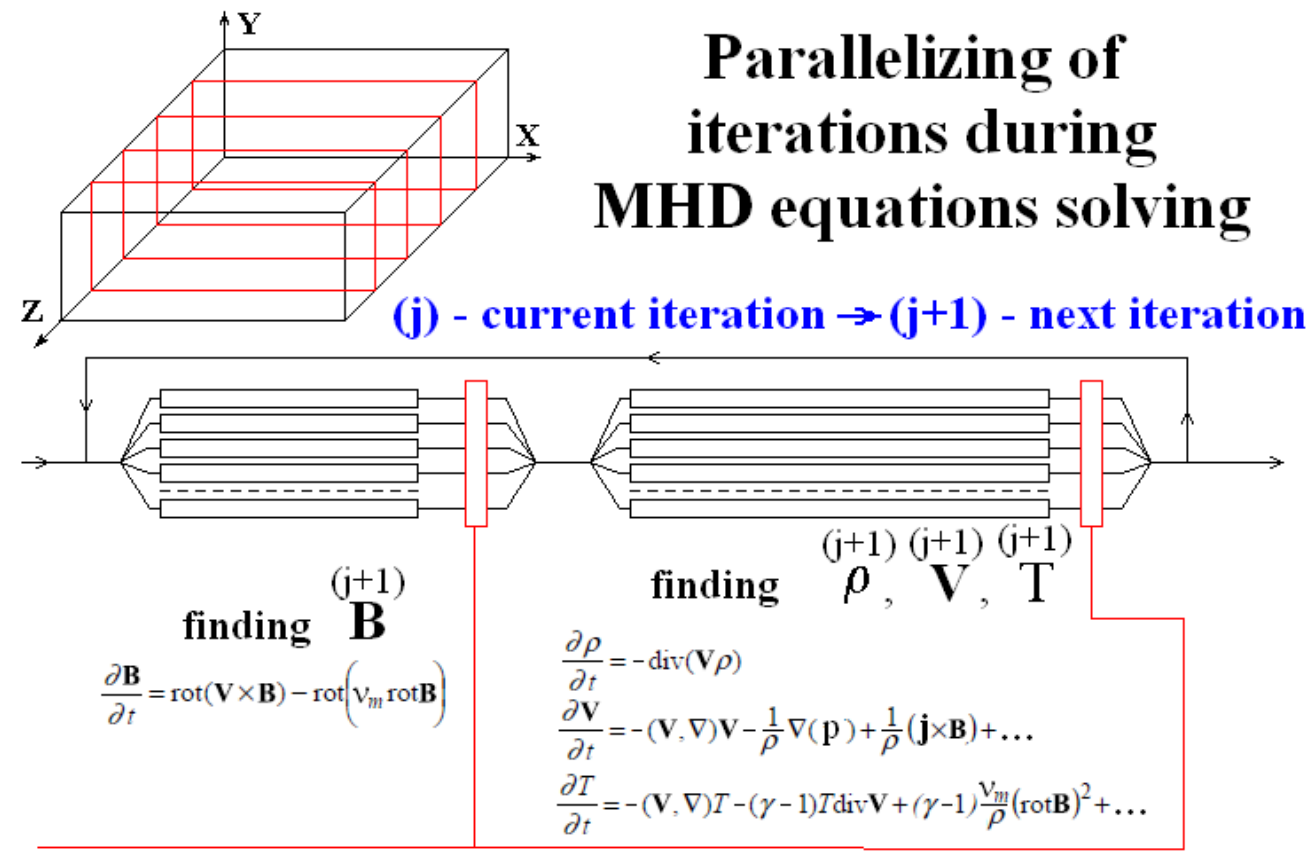

Fig. 2. Parallelization by OpenMP method. Each thread calculates in its own subregion.

The earlier use of the M2050 graphics card made it possible to understand the procedure for parallelizing computations for such difference schemes as the developed scheme for the numerical solution of MHD equations solved by the iteration method using the example of solving the Poisson equation (iterations according to the same principle as for MHD equations, but very much simplified). However, parallel computing using 3D blocks of threads and passing a large number of parameters to the parallelization routines is not possible for the M2050 graphics card. To solve the three-dimensional Poisson equation, parallelization was carried out using one-dimensional blocks, for this purpose a single numbering of all points of the computational grid was introduced. The use of this method did not make it possible to numerically solve a complex system of MHD equations on the M2050 graphics card, and on the Titan-V, V100 and P100 graphics cards it slows down the solution by $\sim 20 \%$.

Parallelization of the numerical solution of MHD equations by the OpenMP method using the computational threads of the main processor is carried out. Intel Visual Fortran was used. The computational domain was divided into subdomains, so that each thread calculates in its subdomain (Fig. 2). Computations on multiprocessor clusters in the clouds showed a slowdown in computation by a factor of several compared to parallel computations on a GPU using CUDA technology. On graphics cards, parallelization is also carried out, similar to the OpenMP method, when each GPU thread computes in its own subdomain (Fig. 2). As a result of such parallelization on a graphics card, the calculation speed becomes $\sim 15$ times slower than the 
calculation speed, according to a program that performs parallel calculations at the points of sequentially selected blocks.

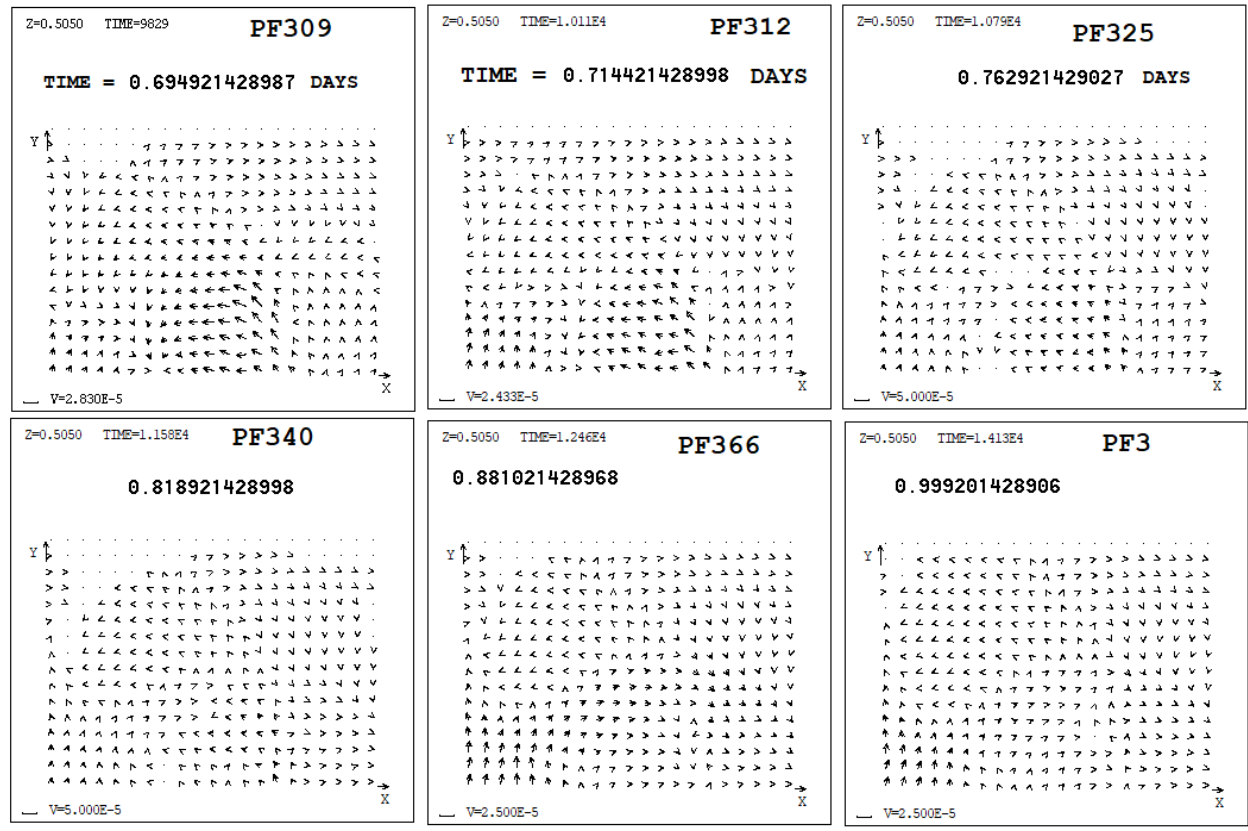

Fig. 4. Evolution of field of plasma velocity in the central plane of computational domain.

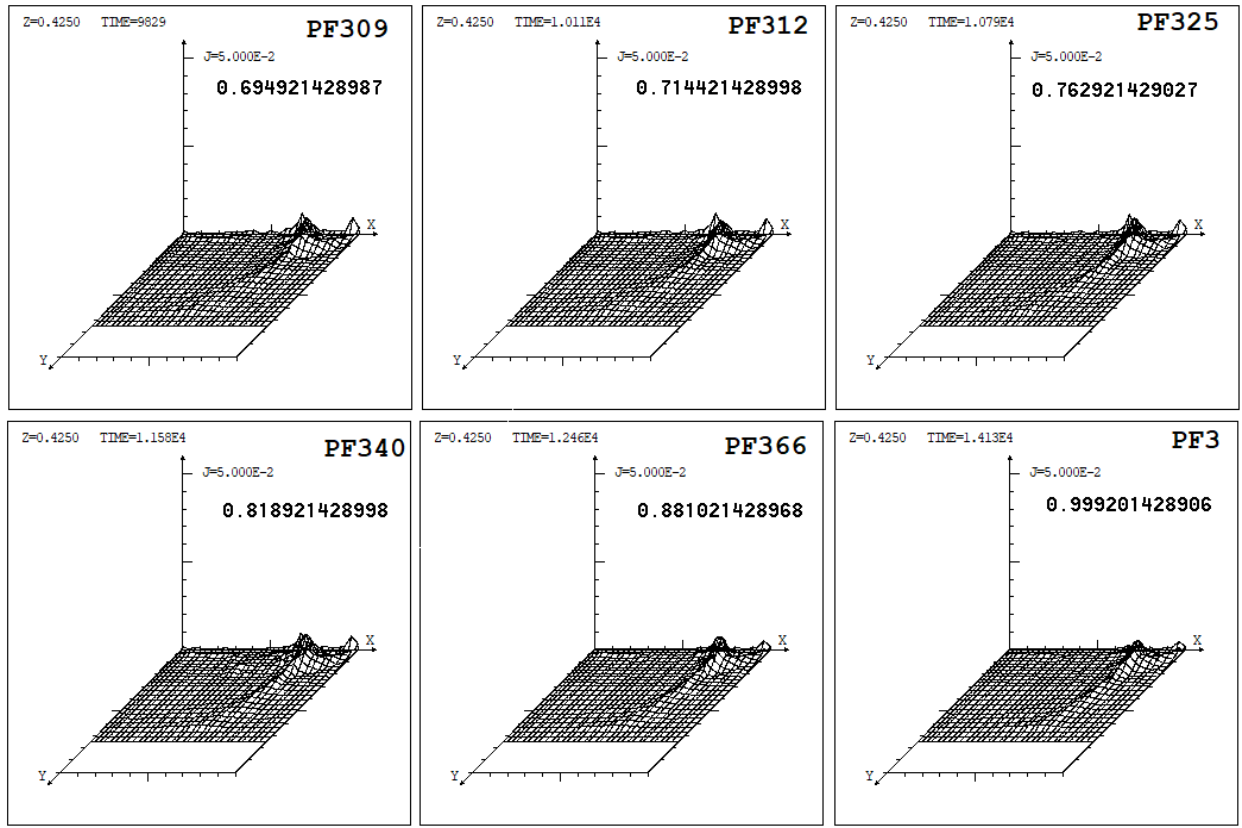

Fig. 5. Evolution of current density distribution.

\section{The presented parallelization methods were used for:}

- subroutines that calculate the values at the given iteration at the points of the threedimensional grid of the computational domain according to the difference scheme used

- calculation at the given iteration (or immediately the final calculation, when possible) of values at the points of the boundary according to the set boundary conditions

- calculating arrays and working with the calculated arrays, necessary to calculate the calculation error at a given iteration in order to determine the end of the iterative process 
- additional calculations using arrays of values in the computational domain (in the corners and on the edges of the computational domain and other additional computations) that do not require parallelization and in previous were calculated by the main computer processor (in order not to send arrays on the graphics card to the main memory, calculations are performed by one computational thread on the graphic card).
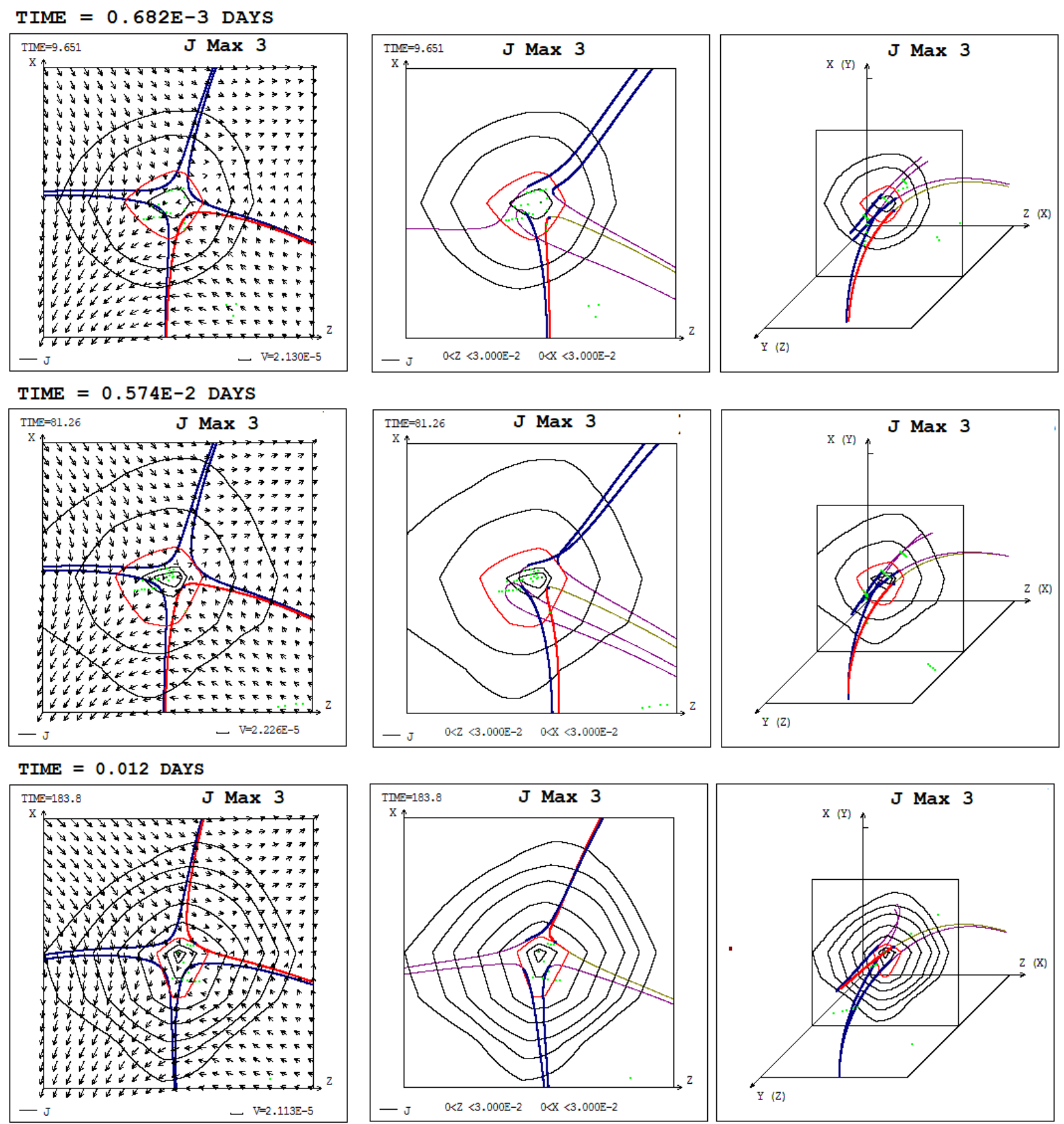

Fig. 6. 2D and 3D magnetic field configurations and plasma flow in current density maximums.

Using the capabilities of the selected computing equipment and software, as well as minimizing the exchange between the GPU memory and the main computer memory [Podgorny et al., 2020], reduced the calculation time of iteration to $0.0282-0.0302 \mathrm{~s}$. Under these conditions, the optimal choice of the parameters of the difference scheme [Podgorny et $a l ., 2020]$ made it possible to calculate the evolution during one day in $\sim 21$ days. For the prognosis, this time should be less than a day, it is necessary to work on further optimization, the reserves are existing. 


\section{Some results of MHD simulation in the real scale of time above AR 10365 for evolution during the day}

The evolution of the plasma velocity and current density in the central plane of computational domain for MHD simulation in the corona above AR 10365 during the first 1.2 days of evolution, when there were no flares yet, is presented in Fig. 4, 5. In some current density maximums, founded by the graphical system of search the magnetic field have X-type configuration and plasma flow should cause the current sheet appearance (Fig. 6).

\section{Conclusion}

1. MHD simulation in the corona above the AR in the real scale of time to study the physical mechanism of a solar flare is possible only using parallel computations.

2. The graphics cards and software which perform fast parallel computing, in particular through the use of three-dimensional blocks of computational threads, have been selected.

3. As a result of the application of parallelization methods, which are feasible for the selected computing equipment and software, and optimization of the parallelization algorithm [Podgorny et al., 2020], the GPU calculation speed exceeded more 120 times the calculation speed on a serial CPU computer (Intel(R) Core(TM) i7 CPU 920 @ 2.67GHz). Calculation in the real scale of time of the evolution of the field and plasma in the corona within one day became possible in 21 days.

4. MHD simulation in the corona above AR 10365 during the first 1.2 days of evolution showed the appearance of X-type configurations with plasma flow, which should cause the formation of a current sheet.

5. Trial calculations showed the possibility of further increasing the speed of calculations using the latest equipment.

\section{Acknowledgments}

The authors are grateful to the SOHO/MDI team for the scientific data provided at $\mathrm{http}: / /$ soi.stanford.edu/magnetic/index5.html, as well as to the many professional cloud service specialists, which made it relatively easy for us to configurate rented remote computers for GPU computing.

\section{References}

Podgorny A.I., Podgorny I.M. (2004) MHD Simulation of Phenomena in the Solar Corona by Using an Absolutely Implicit Scheme. Computational Mathematics and Mathematical Physics. 44, 1784-1806.

Podgorny A.I., I.M. Podgorny I.M. (2008) Formation of Several Current Sheets Preceding a Series of Flares above the Active Region AR 0365. Astronomy Reports. 52, 666-675.

Podgorny A.I, I. M. Podgorny I.M. (2012). Magnetohydrodynamic Simulation of a Solar Flare: 1. Current Sheet in the Corona. Geomagnetism and Aeronomy, 52, 150-161.

Podgorny A.I., Podgorny I.M., Meshalkina N.S. (2017) Magnetic field configuration in corona and X-ray sources for the flare from May 27, 2003 at 02:53. Sun and Geosphere. 12, 85-92.

Podgorny A.I., Podgorny I.M., Meshalkina N. S. (2018) Current sheets in corona and X-ray sources for flares above the active region 10365. Journal of Atmospheric and Solar-Terrestrial Physics. 180, 16-25.

Podgorny A.I., Podgorny I.M., Borisenko A.V. (2020). Study of the solar flare mechanism by MHD simulation in the corona above the active region in real time scale. Proc. 12-th Workshop "Solar Influences on the Magnetosphere, Ionosphere and Atmosphere", Primorsko, Bulgaria, June, 2020. This Issue. 


\title{
Thermospheric Wind Variability at High Latitudes - the Wind Wall
}

\author{
Shepherd, M.G., Shepherd, G.G., Barreto, C.S., Cho, Y.M., Chen, Y.
}

Centre for Research in Earth and Space Science, York University, Canada E-mail: mshepher@yorku.ca

\begin{abstract}
The report provides an overview of previously unrecognized neutral wind reversals in the daytime thermosphere at high latitudes, $50^{\circ}-70^{\circ}$, employing satellite observations by the WINDII experiment on the UARS satellite and the WACCM-X model simulations. The seasonal and local time variability with longitude and height is examined for a number of parameters characterizing the neutral thermosphere and ionosphere. A distinct and very persistent pattern of sharp zonal wind reversals from eastward to westward was observed at $\sim 50^{\circ} \mathrm{E}-200 \mathrm{E}^{\circ}$ in the Southern hemisphere and $280^{\circ} \mathrm{E}-360^{\circ} \mathrm{E}$ in the Northern hemisphere at altitudes from $\sim 150 \mathrm{~km}$ to $300 \mathrm{~km}$, independently of season. The observed westward wind speed reached $-450 \mathrm{~ms}^{-1}$.
\end{abstract}

\section{Introduction}

There have been extensive studies of the neutral thermospheric dynamics at high latitudes but more remains to be explored, in part because of the lack of observations. Richmond et al. (2003) investigated high-latitude winds observed by the Wind Imaging Interferometer (WINDII) on board the Upper Atmosphere Research Satellite (UARS) [Shepherd et al., 2012] and found winds exceeding $300 \mathrm{~ms}^{-1}$ with well-defined correlation with the interplanetary magnetic field $\mathrm{B}_{\mathrm{y}}$ and $\mathrm{B}_{\mathrm{z}}$ components. Thayer et al. (1995) investigated ion and neutral winds observed at Thule, Greenland at $77.5^{\circ} \mathrm{N}$ geographic latitude and found that during disturbed conditions the sunward ion winds reached velocities of $1000 \mathrm{~ms}^{-1}$, while the neutral winds marked velocities of the order of $500 \mathrm{~ms}^{-1}$. Dhadly et al. (2017) combined wind data from many sources to generate neutral wind maps for quiet geomagnetic conditions. A second study by Dhadly et al. (2018) provided such maps for disturbed conditions. Those presentations were polar projections of magnetic latitude as a function of magnetic local time, but did not contain any longitude information.

Recently a series of studies were conducted employing daytime WINDII observations of oxygen airglow emission rates, Doppler temperature and neutral (zonal and meridional) winds and the Whole Atmosphere Community Climate Model including the thermosphere and ionosphere extension (WACCM-X) simulation [Shepherd and Shepherd, 2018; M. Shepherd et al., 2019; Liu et al., 2019] which revealed localized zonal wind reversals from eastward to westward that occurred frequently in the high-latitude thermosphere with strong westward winds reaching velocities of $-450 \mathrm{~ms}^{-1}$ and greater, always at the same longitudes of $50^{\circ} \mathrm{E}-$ $200^{\circ} \mathrm{E}$ in the Southern hemisphere and $280^{\circ} \mathrm{E}-360^{\circ} \mathrm{E}$ in the Northern hemisphere at the same latitude of $\sim 60^{\circ}-70^{\circ}$ in both hemispheres. The zonal wind observations derived from Doppler shifts of the $\mathrm{O}\left({ }^{1} \mathrm{~S}\right)$ green line of atomic oxygen at $557.7 \mathrm{~nm}$ reversed direction from eastward (positive) to westward (negative) so abruptly that Shepherd and Shepherd (2018) suggested the name "wind wall" for this sharp reversal. M. Shepherd et al. (2019) extended the study of those first unique observations by making use of the $\mathrm{O}\left({ }^{1} \mathrm{D}\right)$ red line of atomic oxygen at $630 \mathrm{~nm}$, which occurs at higher altitudes (150-310 km for the WINDII observations) and from which Doppler temperatures can also be obtained. The thermospheric $\mathrm{O}\left({ }^{1} \mathrm{D}\right) 630 \mathrm{~nm}$ airglow emission was chosen because of the information it could provide on the electron density and neutral winds in the F-region of the ionosphere. Data for two Southern Hemisphere seasons, summer solstice and fall equinox were examined.

The present report is intended to present some highlights from these studies to describe the wind wall and together with some new WACCM-X model results to illustrate its dynamics. 
More detailed information on the wind wall morphology can be found in the above mentioned references.

In describing the results that follow and in particular those pertinent to the neutral winds the terms 'peak/maximum' are used to describe the maximum eastward/northward (positive) directions of the zonal/meridional winds. Accordingly, a 'trough/minimum' refers to westward/southward (negative) directions of the zonal/meridional winds.

\section{Results}

\section{a) WINDII thermospheric observations}

The nature of the wind wall in illustrated in Fig. 1 where the observed WINDII zonal winds obtained from the $\mathrm{O}\left({ }^{1} \mathrm{~S}\right)$ emission are shown as a function of longitude and height over a period of several consecutive days. The left panel (A) shows the zonal wind during DoY (day of year) 73 - 79 (March $13-19$ ), 1996 at $60^{\circ} \mathrm{S}-70^{\circ} \mathrm{S}$, while the right panel (B) shows the same for DoY $275-280$ (October $2-7$ ), 1995 at $60^{\circ} \mathrm{N}-70^{\circ} \mathrm{N}$.
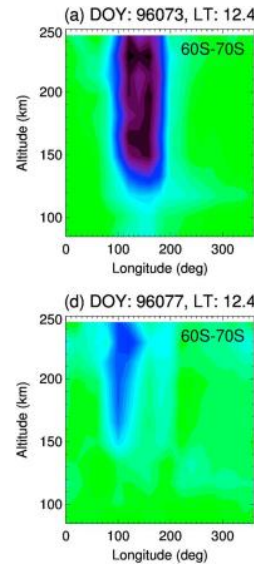

A)
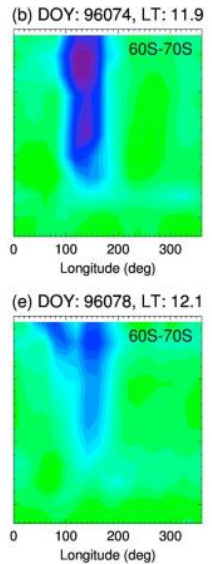

$100 \quad 200$
Longitude (deg)

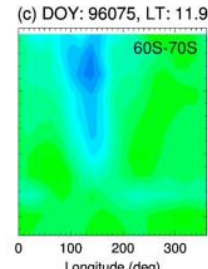

(f) DOY: 96079, LT: 12.5

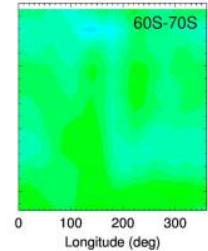

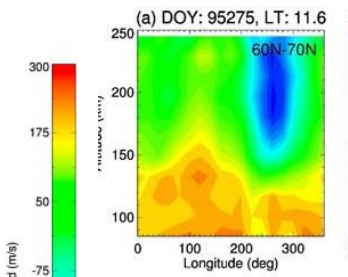

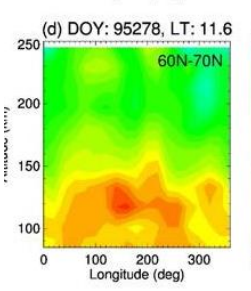

B)
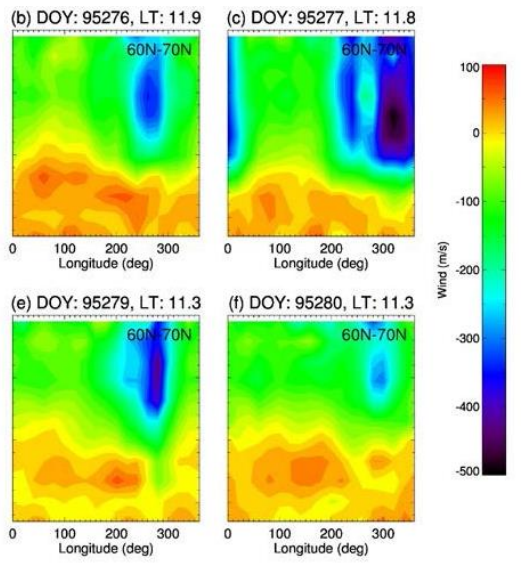

Fig. 1: WINDII zonal wind from the $O\left({ }^{1} S\right)$ emission as a function of longitude and altitude for: $(A) D o Y$ 73 - 79 (March 13 - 19), 1996 (left panel) at 60' $\mathrm{S}-70^{\circ} \mathrm{S}$, and (B) DoY 275 - 280 (Oct 2 -7), 1995 at $60^{\circ} \mathrm{N}-70^{\circ} \mathrm{N}$.

Here and elsewhere the convention is that eastward zonal wind is positive, while westward zonal wind is negative. As can be seen the reversal of the zonal wind from predominantly eastward to westward persistently occurs around $50^{\circ} \mathrm{E}-200^{\circ} \mathrm{E}$ in the Southern hemisphere and at around $280^{\circ} \mathrm{E}-360^{\circ} \mathrm{E}$ in the Northern hemisphere. Although it varies in magnitude and altitude extension from day to day, its boundaries are sharp and extend from $\sim 150 \mathrm{~km}$ to at least $250 \mathrm{~km}$ height, based on the $\mathrm{O}\left({ }^{1} \mathrm{~S}\right)$ emission and wind observations. The local times of these zonal wind reversals for this period are around noon, 12 LT.

Fig. 2 shows the WINDII meridional wind for the period of DoY 96073 - 96079 and latitude band of $60^{\circ} \mathrm{S}-70^{\circ} \mathrm{S}$. Following the convention, here the positive values correspond to northward direction, while the negative values are for southward direction. Similar to the zonal wind, the meridional wind also shows sharp reversal of direction over the same longitude range of $\sim 50^{\circ} \mathrm{E}$ to $200^{\circ} \mathrm{E}$. Its longitudinal extension is broader than that of the zonal wind and appears accompanied by a northward wind peak east of the southward (negative) peak. The meridional wind is weaker than the zonal wind, ranging within $\pm 200 \mathrm{~ms}^{-1}$, compared to $-450 \mathrm{~ms}^{-1}$ to 300 $\mathrm{ms}^{-1}$. Examining a series of zonal wind vertical profiles along a WINDII orbit traversing the $100^{\circ} \mathrm{E}-200^{\circ} \mathrm{E}$ region at $60^{\circ} \mathrm{S}-70^{\circ} \mathrm{S}$ on DoY 96073 for example (not shown here), showed that while profiles on the edge of the zonal wind reversals were almost vertical with wind velocity 
of $\sim 0 \mathrm{~ms}^{-1}$, within the reversal zone individual wind profiles indicated westward wind speeds of up to $-650 \mathrm{~ms}^{-1}$, with most of the observations of $-450 \mathrm{~ms}^{-1}$.

WINDII observations of the upper thermosphere daytime fields of $\mathrm{O}\left({ }^{1} \mathrm{D}\right)$ volume emission rate (VER), neutral zonal and meridional winds and Doppler temperature from $170 \mathrm{~km}$ to 310 $\mathrm{km}$ were also available providing an opportunity to examine the morphology of the wind wall to higher altitude. Fig. 3 shows some of these observations as a function of longitude and altitude in the Southern hemisphere at $50^{\circ} \mathrm{S}-70^{\circ} \mathrm{S}$. The two days shown are representative of a series of consecutive days of observations during March/April 1994 (fall equinox), (left column) and January 1995 (summer solstice), (right column) corresponding to local time of 8 $-13 \mathrm{LT}$ and $12-16 \mathrm{LT}$, respectively.
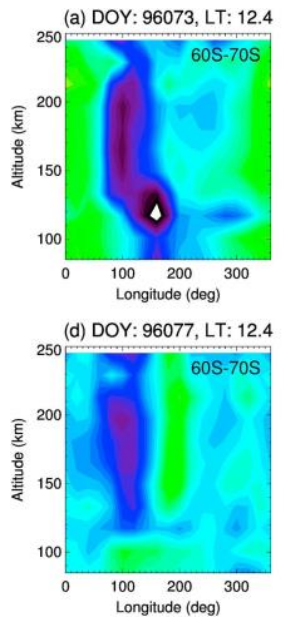
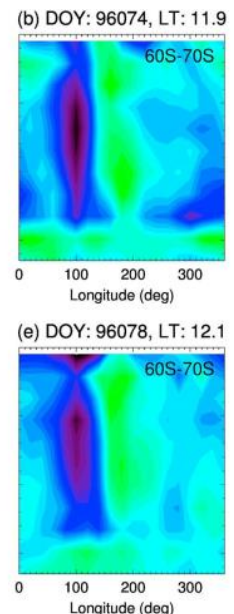
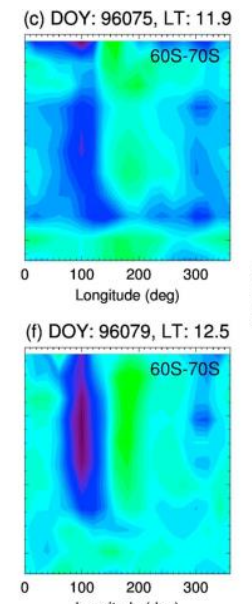

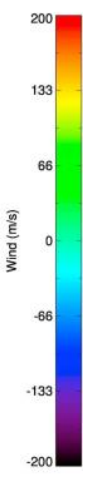

Fig. 2: Meridional wind for the same period as in Fig. 1. Note that for day 79 in panel $2 f$ there is a strong wind wall signature although it does not exist for the zonal wind in Fig. If.

What is remarkable in these plots is the fact that all fields considered reach peak at the same longitude range of about $50^{\circ} \mathrm{E}$ to $200^{\circ} \mathrm{E}$ independently of season (fall equinox or summer solstice) or local time (morning vs afternoon). At the time of the peak $\mathrm{O}\left({ }^{1} \mathrm{D}\right)$ VER used as a proxy for electron density, around $220-240 \mathrm{~km}$ height the zonal wind reversed direction from prevailing eastward to westward. During fall equinox the westward zonal wind reached velocity of $-330 \mathrm{~ms}^{-1}$. The meridional wind also showed an abatement or reversal of direction to southward around $100^{\circ} \mathrm{E}$, accompanied with an increase/reversal to poleward around $200^{\circ} \mathrm{E}$. This pattern is very similar to the $\mathrm{O}\left({ }^{1} \mathrm{~S}\right)$ meridional wind observations in Fig.2. Finally, the $\mathrm{O}\left({ }^{1} \mathrm{D}\right)$ temperature showed a peak/trough pattern with a broad peak centred around $100^{\circ} \mathrm{E}$, increasing in magnitude with height and a trough between $200^{\circ} \mathrm{E}$ and $300^{\circ} \mathrm{E}$, here more pronounced in the DoY 95004 observations.

\section{b) WACCM-X simulations of the wind wall}

WACCM-X [Liu et al., 2018, and references therein] is a comprehensive numerical model, which produces neutral atmosphere 3-D temperature, density, winds, composition, ionospheric parameters and electric potential fields. It is a part of the NCAR Community Earth System Model and extends the description of the neutral atmosphere up to $500-700 \mathrm{~km}$ altitude. The thermospheric and ionospheric components of WACCM-X add neutral species composition, electron and ion density, as well as ion and electron temperatures. The most notable additions are the fully coupled low and middle latitude electrodynamics, dynamical transport of $\mathrm{O}^{+}$and high latitude forcing by the magnetospheric electric fields and specific heat [Liu et al., 2018]. The effects of geomagnetic activity are incorporated in WACCM-X by applying the Heelis empirical convection pattern, based on the geomagnetic Kp index. Fig. 4 shows maps of 
WACCM-X simulations of electron density, neutral temperature, zonal and meridional winds at $200 \mathrm{~km}$ height for the latitude band of $50^{\circ} \mathrm{N}-80^{\circ} \mathrm{N}$, on DoY 95278 (October 5, 1995). The results are for the "specified dynamics" version of the model constrained by the meteorology from the Modern-era Retrospective Analysis for Research and Application 2 (MERRA2) [Liu et al., 2018 and references therein]. For the simulations presented here the model was run on the Niagara supercomputer SciNet (hhtp://www.scinethpc.ca/niagara/).

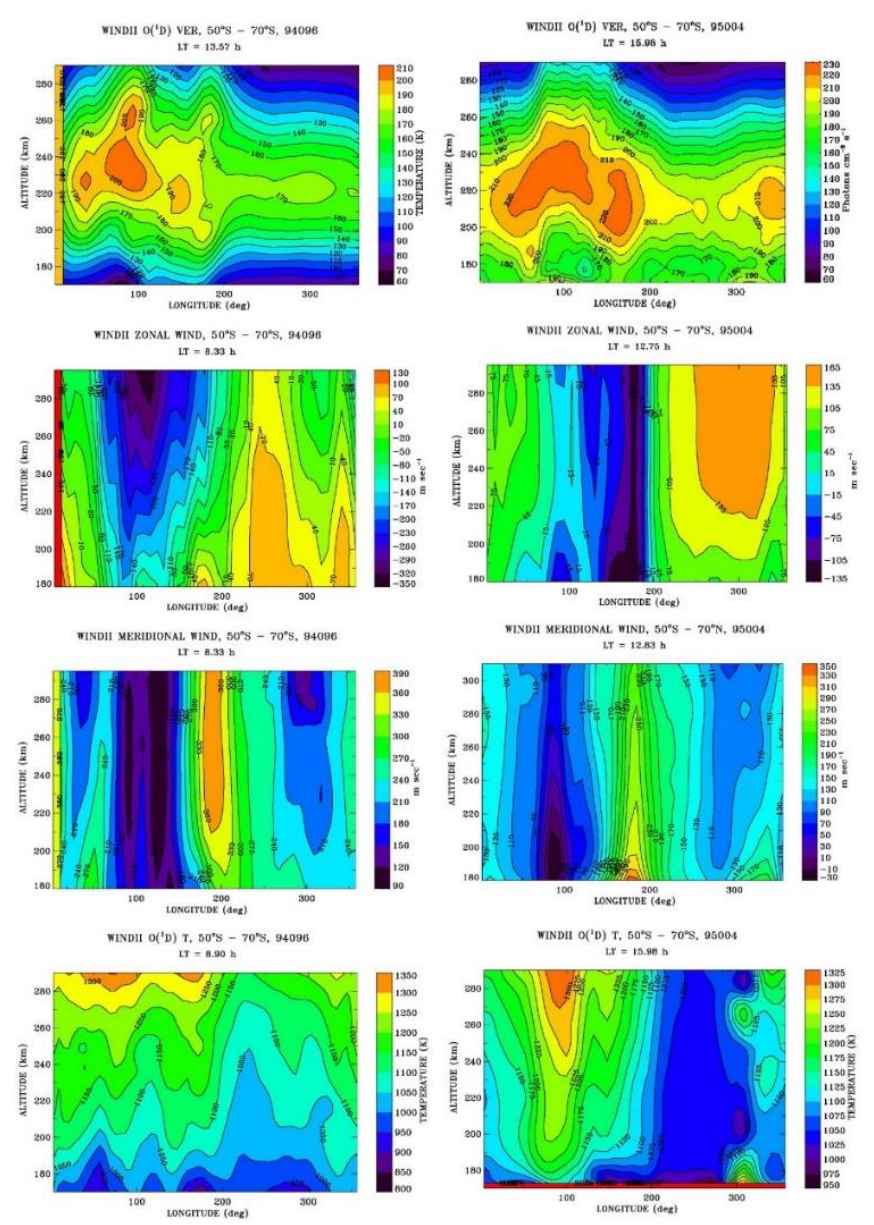

Fig. 3: WINDII $O\left({ }^{1} D\right)$ VERs, neutral winds and Doppler temperature at $50^{\circ} \mathrm{S}-70^{\circ} \mathrm{S}$ as a function of longitude and altitude for April 6, 1994 (DoY 94096) (left column) and January 4, 1995 (DoY 95004) (right column).

From Fig. 4 it is easy to see that the westward zonal wind follows closely the crest of the electron density along the auroral oval with the geomagnetic pole in 1995 at $79.4^{\circ} \mathrm{N}$ and $288.6^{\circ} \mathrm{E}$, and appears to be on the poleward side of the electron density crest, which in turn outlines the auroral oval. DoY 95277 (October 4, 1995) shown here is the same, shown in Fig 1c, right panel (B), with Ap of 51. The reader is reminded that while the plots in Figs. $1 \& 2$ are composites of all observations within the $\pm 60^{\circ}-70^{\circ}$ latitude band, as a function of longitude and height, Fig. 4 shows the mapping of the respective parameters in longitude and latitude at a selected height, e.g. $200 \mathrm{~km}$. The westward wind reached velocity of $-550 \mathrm{~ms}^{-1}$ at its peak around $350^{\circ} \mathrm{E}-50^{\circ} \mathrm{E}$ and $\sim 300-350 \mathrm{~ms}^{-1}$ at $\sim 250^{\circ} \mathrm{E}-330^{\circ} \mathrm{E}$, in agreement with the WINDII observations. There is about a $200 \mathrm{~K}$ temperature variation throughout the latitude band considered, with a cold temperature anomaly around $100^{\circ} \mathrm{E}$ extending poleward to $\sim 67^{\circ} \mathrm{N}$ that is similar to the anomaly seen in Fig. 3 (the bottom row). The meridional wind peaks around $150^{\circ} \mathrm{E}-300^{\circ} \mathrm{E}$ poleward of the auroral oval. 

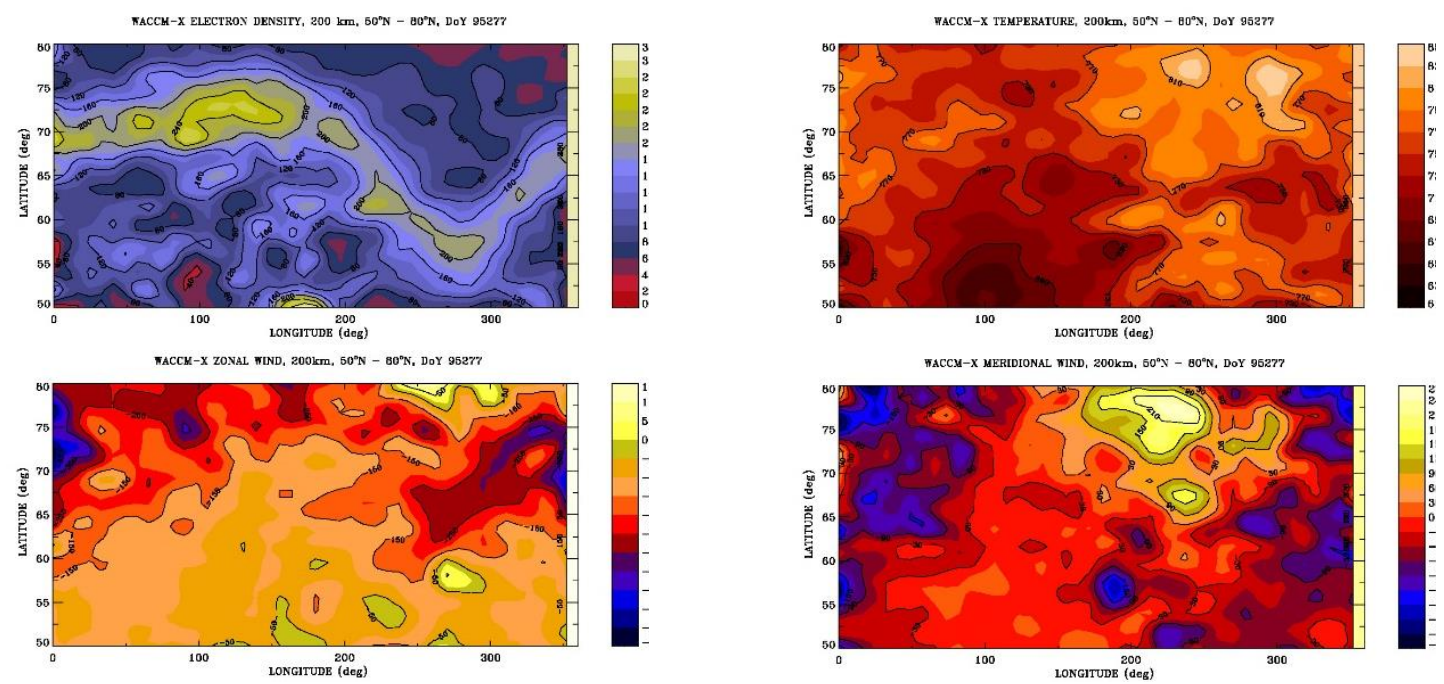

Fig. 4: WACCM-X model simulations of electron density, temperature and neutral (zonal and meridional) winds at $50^{\circ} \mathrm{N}-80^{\circ} \mathrm{N}$ as a function of longitude at $200 \mathrm{~km}$ height for October 4,1995 (DoY 95277).

\section{Discussion}

One of the first reports on the longitudinal variability of the neutral atmosphere at Southern middle and high latitudes was by Laux and von Zahn (1979) employing monopole mass spectrometer data from the Esro 4 satellite. They reported drastic changes in the $[\mathrm{O}] /\left[\mathrm{N}_{2}\right]$ ratio observed between $50^{\circ} \mathrm{S}-80^{\circ} \mathrm{S}$ and $110^{\circ} \mathrm{E}$ to $180^{\circ} \mathrm{E}$ geographic, within the vicinity of the geomagnetic pole, but shifted by $\sim 10^{\circ}$ in latitude toward the equator. As is presented in the current report the $\mathrm{N}_{2}$ and $\mathrm{O}$ extreme variations occurred at the same longitude range independently of local time, remaining fixed to a limited longitude band. The persistence of the observed phenomenon and the lack of strong correlation with geomagnetic activity led Laux and von Zahn (1979) to the conclusion that this is a regular feature associated with thermospheric air motion.

Various studies on the seasonal and longitudinal variability of ionospheric daytime electron density have shown that it exhibits variability, related to changes in the thermo-spheric composition, in particular of the $[\mathrm{O}] /\left[\mathrm{N}_{2}\right]$ ratio. Millward et al (1996) proposed a theory to explain the observed seasonal and semi-annual variations of electron density only through the longitudinal dependence of the interface between the solar-driven low/midlatitude neutral atmosphere circulation and the geomagnetically driven high latitude circulation, where the latitude of the interface depends on longitude. In the vicinity of the magnetic pole the magnetically driven circulation extends to relatively low geographic latitudes, and on the dayside its interaction with the neutral atmosphere circulation occurs at a geographic latitude of about $\sim 50^{\circ}-65^{\circ}$. As was shown there is a longitudinal "wind wall" at about $\pm 63^{\circ}-66^{\circ}$ in both hemispheres where the neutral wind velocity decreases and/or reverses direction affecting the variability of the oxygen airglow $\left(\mathrm{O}\left({ }^{1} \mathrm{~S}\right), \mathrm{O}\left({ }^{1} \mathrm{D}\right)\right)$, temperature and $[\mathrm{O}]$ observed. An interesting feature in the observed variability was the fact that the peaks of all parameters considered (the $\mathrm{O}\left({ }^{1} \mathrm{~S}\right)$ and $\mathrm{O}\left({ }^{1} \mathrm{D}\right)$ VER, temperature, zonal and meridional winds) in the vicinity of the magnetic pole were a persistent longitudinal feature independent of season and daytime local time. This is even more striking considering the fact that the observations are for different although consecutive days and different local times determined by the precession of the WINDII/UARS orbit. Given the fact that the geomagnetic pols in 1995 were at $79^{\circ} \mathrm{S}$ and $109^{\circ} \mathrm{E}$ in the Southern hemisphere and $79^{\circ} \mathrm{N}, 289^{\circ} \mathrm{E}$, in the Northern hemisphere, they are very closely 
positioned to the regions where the reversal of the zonal wind and the wind wall are observed. The connection of the thermospheric wind reversals to the geomagnetic field and further coupling to the magnetosphere requires further investigation at latitudes higher than are available from WINDII, and so this will be a subject of future studies.

\section{Conclusions}

1. Between longitudes of $50^{\circ} \mathrm{E}-200^{\circ} \mathrm{E}$ in the Southern hemisphere and $280^{\circ} \mathrm{E}-360^{\circ} \mathrm{E}$ in the Northern hemisphere and at $60^{\circ}-70^{\circ}$ latitudes there is a wind wall of extreme velocity reversals to westward zonal and southward meridional winds regularly $-450 \mathrm{~ms}$ ${ }^{1}$ and $-200 \mathrm{~ms}^{-1}$, respectively. Zonal winds could reach velocities of up to $-650 \mathrm{~ms}^{-1}$, observed when WINDII's most poleward orbit (at $72^{\circ}$ geographic, $82^{\circ}$ geomagnetic), cuts through the polar cap extreme wind field. It was suggested that the wall is the boundary between the strong westward polar cap winds and the weaker eastward winds equatorward of that.

2. Comparisons between the WINDII observations and WACCM-X simulations are in generally good agreement in describing the wind wall. The model simulations have shown that the appearance of the wind wall is modulated by the solar and geomagnetic activity. For high solar and geomagnetic active periods the wind wall is very pronounced and the westward zonal winds within the wall region are very strong.

\section{References}

Dhadly, M. S., Emmert, J. T., Drob, P. D., et al. (2017). Seasonal dependence of northern high latitude upper thermospheric winds: A quiet time climatological study based on ground-based and space-based measurements. J. Geophys. Res.: Space Phys., 122, 2619-2644. https://doi.org/10.1002/2016JA023688

Dhadly, M. S., Emmert, J. T., Drob, P. D., et al. (2018). Seasonal dependence of geomagnetic active-time northern high-latitude upper thermospheric winds. J. Geophys. Res.: Space Phys., 123(1), 739-754. https://doi.org/10.1002/2017JA024715

Laux, U., \& von Zahn, U. (1979). Longitudinal Variations in Thermospheric Composition Under Geomagnetically Quiet Conditions. J. Geophys. Res., 84 (A5), 1942 - 1946.

Liu, H.-L., Bardeen, C. G., Foster, B. T., et al. (2018). Development and validation of the whole atmosphere community climate model with thermosphere and ionosphere extension (WACCM-X 2.0). J. Adv. Model. Earth System, 10, 381- 402. https://doi.org/10.1002/2017MS001232

Liu, S., Shepherd, G. G., Chen, Y., Shepherd, M. G., \& Bhutia, S. (2019). WINDII observations and WACCM-X simulations of high-latitude winds under different solar radio flux and geomagnetic disturbance conditions. J. Geophys. Res.: Space Physics, 124, 6087-6096. https://doi.org/10.1029/2019JA026864

Millward, G. H., Moffett, R.J., Quegan, S., \& Fuller-Rowell, T.J. (1996). Ionospheric F2 layer seasonal and semiannual variations. J. Geophys. Res., 101, 5149 - 5156, https://doi.org/10.1029/95JA03343.

Richmond, A. D., Lathuillère, C., \& Vennerstroem, S. (2003). Winds in the high-latitude lower thermosphere: Dependence on the interplanetary magnetic field. J. Geophys. Res., 108(A2), 1066. https://doi.org/ 10.1029/2002JA009493

Shepherd, G.G., Thuillier, G., Cho, Y.-M., et al. (2012). The wind imaging interferometer (WINDII) on the upper atmosphere research satellite: A 20 year perspective. Rev. Geophys., 50, RG2007 https://doi.org/ 10.1029/2012RG000390.

Shepherd, G. G., \& Shepherd, M. G. (2018). High-latitude observations of a localized wind wall and its coupling to the lower thermosphere. Geophys. Res. Lett., 45, 4586-4593. https://doi.org/10.1029/2018GL077722

Shepherd, M., Shepherd, G., \& Codrescu, M. (2019). Perturbations of O $\left({ }^{1} \mathrm{D}\right)$ VER, temperature, winds, atomic oxygen, and TEC at high southern latitudes. J. Geophys. Res.: Space Phys., 124. https://doi.org/10.1029/ 2019JA026480

Thayer, J. P., Crowley, G., Niciejewski, R. J., et al. (1995). Ground-based observations of ion/neutral coupling at Thule and Qânâq, Greenland. J. Geophys. Res., 100, 12,189 - 12,199. https://doi.org/10.1029/95JA00131 


\title{
Study of the Solar Flare Mechanism by MHD Simulation in the Corona Above the Active Region in Real Time Scale
}

\author{
Podgorny A.I. ${ }^{1}$, Podgorny I.M. ${ }^{2}$, Borisenko A.V. ${ }^{1}$ \\ ${ }^{1}$ Lebedev Physical Institute of the RAS, Moscow, Russia \\ ${ }^{2}$ Institute of Astronomy of the RAS, Moscow, Russia \\ E-mail: podgorny@ lebedev.ru
}

\begin{abstract}
.
Observations of soft X-ray emission and of highly ionized iron lines indicate the appearance of a solar flare in the corona at altitudes of $15000-30000 \mathrm{~km}$. The slow accumulation of magnetic energy in the corona above the active region and its subsequent fast release during the flare is explained by the accumulation of energy in the magnetic field of the current sheet formed in the vicinity of X-type singular line. Since the configuration of the magnetic field in the corona cannot be determined from observations, to study the flare situation above the active region, a numerical magnetohydrodynamic (MHD) simulation is carried out in which the magnetic field observed on the photosphere is used as the boundary condition. Despite specially developed numerical methods, MHD simulation takes a rather long time, and therefore it was previously performed only on a greatly reduced (10 000 times) time scale. The results were obtained on the study of the solar flare mechanism, but the magnetic field configuration was distorted due to the unnaturally rapid change in the field on the photosphere. For a more accurate study of the solar flare mechanism, simulation in the real scale of time was performed, which was made possible through the use of parallel calculations on the 'home supercomputer'. The first results of MHD simulation in the real scale of time above the AR 10365 showed the appearance of a plasma flow near singular X-type lines, which have to cause to the formation of a current sheet.
\end{abstract}

\section{Introduction. Solar flare mechanism and its observational manifestations.}

Solar flares are the most powerful manifestations of solar activity, during which energy of $10^{32} \mathrm{erg}$ is released in a few tens of minutes. Since flares occur above active regions (ARs) with a large magnetic field (the value of the field in the active region on the solar surface reaches several thousand $\mathrm{G}$ ), there is now no doubt that magnetic energy is released during flares. The primordial energy release from the flare occurs high in the solar atmosphere (in the lower corona) at altitudes of $15,000 \mathrm{~km}-30,000 \mathrm{~km}$, which is $\sim 1 / 40-1 / 20$ of the solar radius. First of all, this has been proven by direct measurements of the thermal X-ray emission of flares on the limb [Lin et al., 2003]. Evidence of the appearance of a flare in the corona is also the invariability of the magnetic field on the solar surface [Podgorny et al., 2015], and the observation of ultraviolet radiation in the lines of multiply ionized iron ions, which can be used to judge the change in plasma temperature in time at the site of the flare [Podgorny and Podgorny 2018].

The main flare process high in the corona can be explained by the mechanism of S.I. Syrovatskii [Syrovatskii 1966]: the accumulation of magnetic energy in the field of a current sheet, which is formed in the vicinity of a X-type singular line of magnetic field. As a result of quasi-stationary evolution, the current sheet transfers into an unstable state. Instability causes a flare release of energy with all the observed manifestations of a flare, which are explained by the electrodynamic model of a flare proposed by I.M. Podgorny [Podgorny et al., 2010]. The model was developed based on the results of observations and numerical MHD simulation and uses analogies with the electrodynamic substorm model proposed earlier by the author on the basis of Intercosmos-Bulgaria-1300 satellite data [Podgorny et al., 1988]. The hard X-ray beam radiation on the surface of the sun during a flare is explained by the deceleration in the lower 
dense layers of the solar atmosphere of electron fluxes accelerated in field aligned currents caused by the Hall electric field in the current sheet.

Since the configuration of the magnetic field in the corona cannot be obtained from observations, in order to study the physical mechanism of the flare, as well as improve the prognosis of flares, it is necessary to carry out magnetohydrodynamic (MHD) simulation in the corona above the active region (AR), in which all conditions are taken from observations. When performing MHD simulations, no assumptions about the flare mechanism were made at setting of the problem [Podgorny and Podgorny 2008], the purpose of the simulation was to determine the mechanism of the solar flare. For setting the conditions, we used the magnetic field distribution observed in the photosphere; the other conditions were approximated by the free exit conditions. In order to speed up the calculation, a finite-difference scheme was specially developed, which had to remain stable for the largest possible time step [Podgorny and Podgorny 2007, 2008]. The scheme was realized in the PERESVET program. The scheme is upwind, absolutely implicit, and conservative with respect to the magnetic flux, it is solved by the iteration method. Despite the use of specially developed methods, it was possible to carry out MHD simulations in the corona on a usual computer only on a greatly reduced (by a factor of $10^{4}$ ) time scale. At the same time, instability arose at the photospheric boundary, caused by an unnaturally rapid change in the magnetic field, however, thanks to the application of the developed methods, it did not propagate into the calculated region of the corona and did not increase to infinity. In order to get rid of this instability, as well as to obtain the correct development of processes in time, it is necessary to carry out MHD simulations in real scale of time.

In view of the impossibility of MHD simulation in the real scale of time in the corona in the foreseeable time [Borisenko et al., 2020], it is necessary to carry out parallel computations.

\section{Choosing the numerical method for solving MHD equations and optimization of the parallelization algorithm}

To increase the computation speed when carrying out parallel computations, the choice of the numerical method should be such as to maximize the time step at which the difference scheme remains stable and the number of iterations at each time step was minimal. In our case, the choice of the numerical method means the choice of the type of the difference scheme within a given type of schemes, which are absolutely implicit and conservative with respect to the magnetic flux, and the choice of the parameters of the difference scheme, first of all, the usual and magnetic artificial viscosity, which is used mainly near the boundary (where difficulties always arise with the correct setting of all boundary conditions). In addition, the parallelization algorithm has been optimized for these purposes. Also, to increase the computation speed, the computation time of one iteration was reduced [Borisenko et al., 2020] due to the correct choice of computing equipment and software and the use of the capabilities of the selected equipment for parallelizing the computation.

For implicit finite-difference scheme time step $\tau$ in principle can be larger than the time from Courant condition $\tau_{\mathrm{K}}: \tau<\mathrm{h} /\left(\mathrm{V}_{\mathrm{MV}}+\mathrm{V}_{\mathrm{MA}}\right)$. Here $\mathrm{h}$ is space step, $\mathrm{V}_{\mathrm{MV}}$ is the maximal of absolute value of velocity and $\mathrm{V}_{\mathrm{MA}}$ is the maximum of absolute values of magnetosonic and Alven velocities.

Calculations showed, that time for proposed difference scheme step $\tau$ must be less then $\tau_{K}$, in spite of that the absolute implicit scheme is used. Apparently, it is due to that the system of equations with cross-terms. Also it can be due to solving of implicit scheme by the iteration method, and the first iteration is in fact the solution of explicit scheme. To solve the proposed implicit difference scheme without using the iteration method, the alternating direction method should be applied. However, with a sufficiently large acceleration by the magnetic tension force 
(which appears at the place of plasma outflow from the current sheet), the use of the alternating direction method leads to numerical instabilities even for the Courant time step.

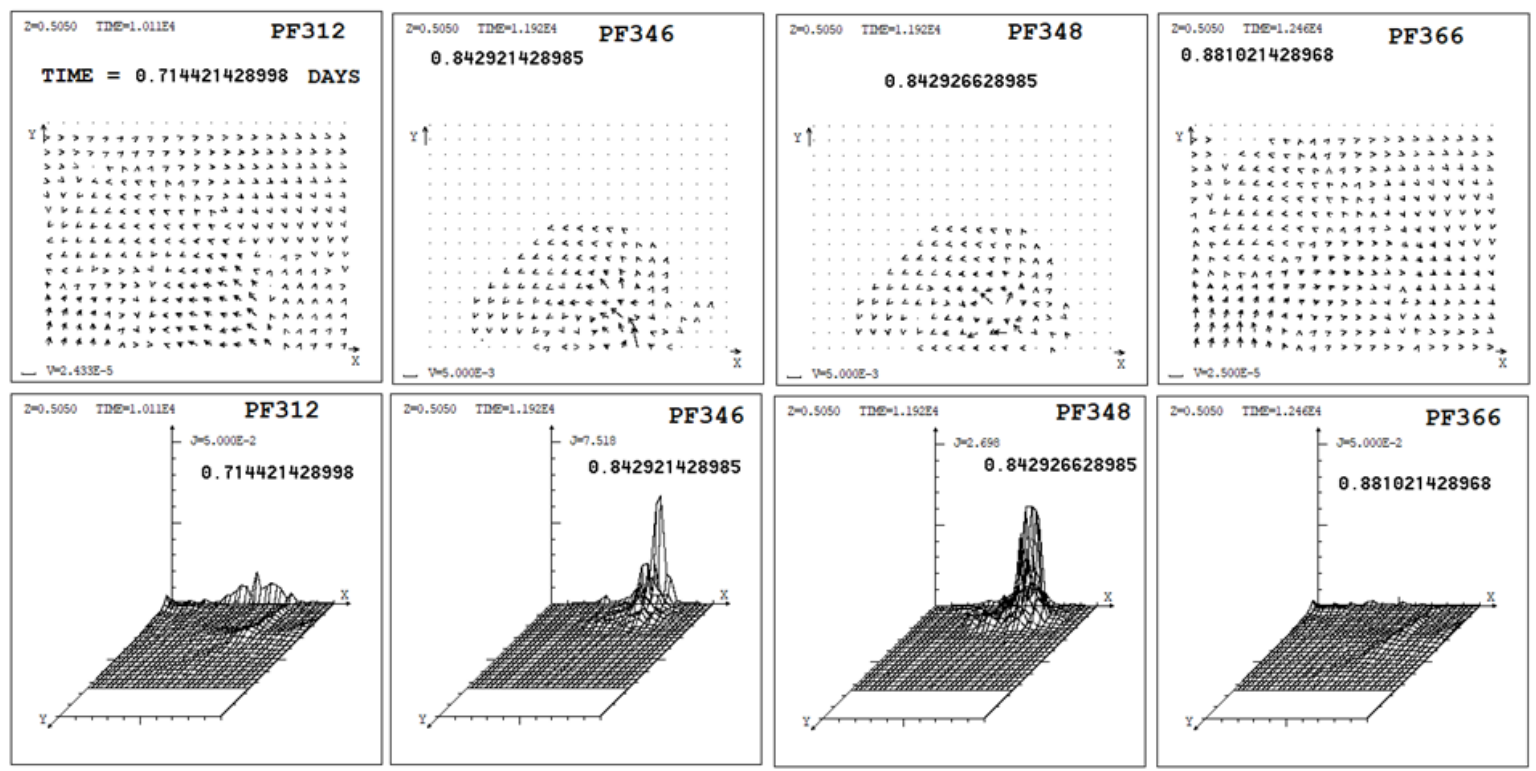

Fig. 1. The appearance and disappearance of instability near the photospheric boundary

It is difficult to choose the most optimal parameters during the calculation, because there are several parameters and the step $\tau_{\mathrm{K}}$ changes during the calculation. Calculations have shown that the currently used set of parameters is quite optimal:

- Time step $\tau=0.410^{-7}$ days (Courant step $\tau_{\mathrm{K}}$ varies from $0.45 \times 10^{-7}$ to $0.79 \times 10^{-7}$ in the process of calculation, and depending on the details of the rules for its determination near the boundary)

- Artificial viscosity (usual and magnetic) $v=310^{-3}$.

- Precision of solution of implicit scheme $\varepsilon=10^{-7}$, at which 3 iterations are performed. In this case, in order to avoid instability, from time to time it is necessary to set for a short interval $\left(\sim 100\right.$ time steps) $\varepsilon=10^{-10}$, for which $\sim 60$ iterations will be performed.
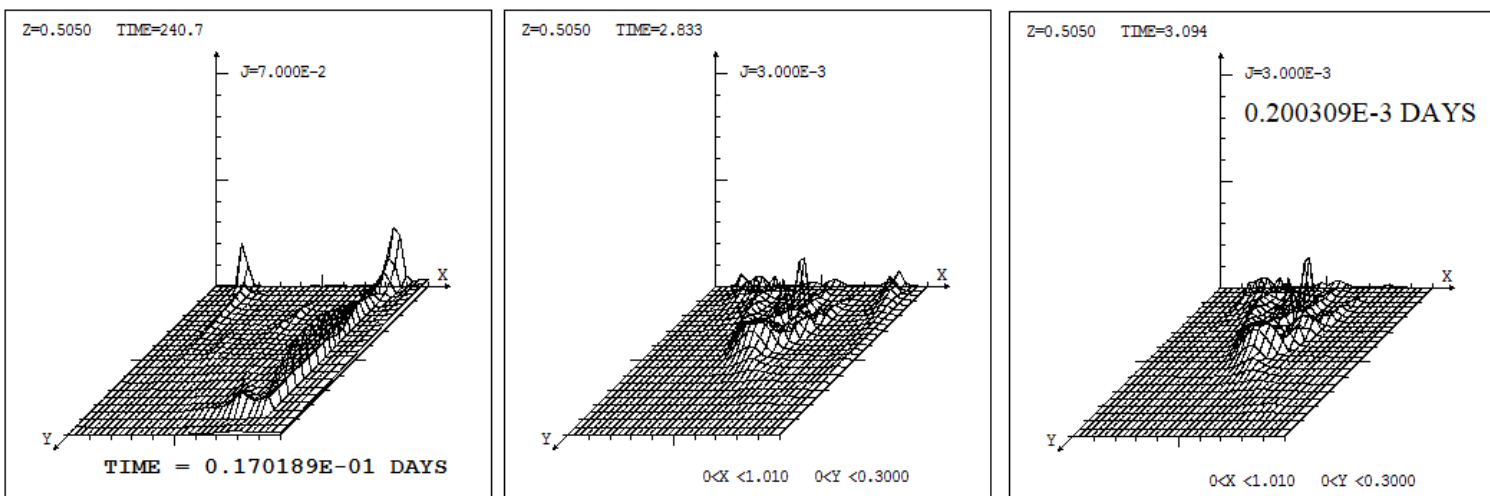

Fig. 2. Current density in the central plane of computational domain. Propagation, initialization and stabilizing instability on the non-photospheric boundary

In order to optimize of the parallelization algorithm minimization of transfers of arrays of distributions of all values in the computational domain and auxiliary arrays, between the memory on the graphics card (arrays with the DEVICE attribute) and the main computer 
memory was performed. After all the upgrades, during the entire numerical solution of the MHD equations, there is no transfer between the memory of the graphic card and the main memory of the computer (such transfer cannot be avoided only when the calculation results are written to a file, it can be carried out only from the main memory, and occurs every several thousand steps, so it almost does not take calculation time).
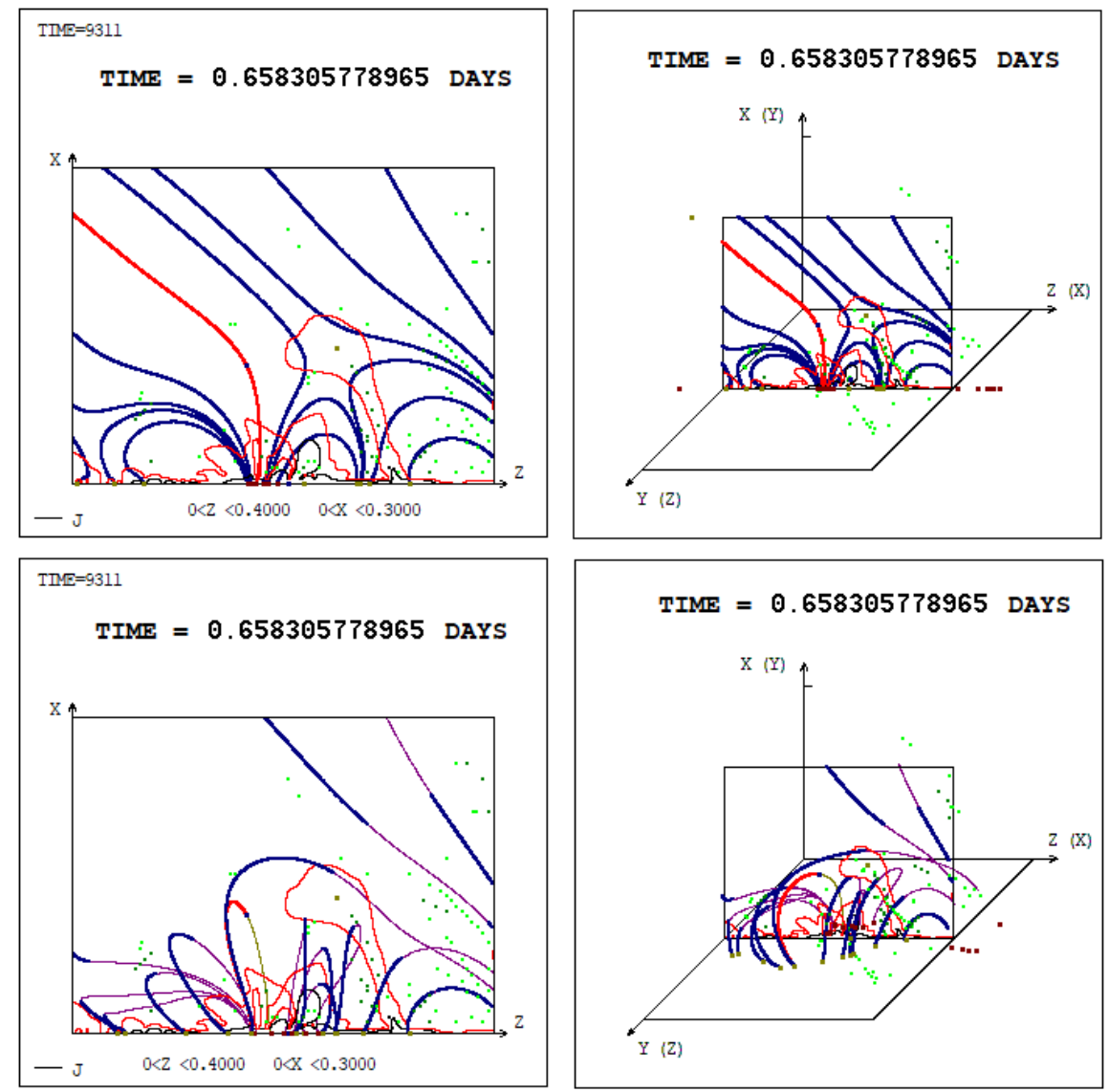

Fig. 3. Magnetic configuration in main part of computational domain of corona in $3 D$ space and in the central plane. Local current density maximums, which are the candidates on the places of flares are shown as the green points.

As a result of choosing the numerical method for solving MHD equations and optimization of the parallelization algorithm and also due to the correct choice of computing equipment and software [Borisenko et al., 2020] and the use of the capabilities of the selected equipment for parallelizing the calculation, now we can get the time for calculation the evolution during the day above the AR, equal to 21 days. Under less favorable conditions it can be increased by $7 \%$ $-10 \%$. For the prognosis, this time should be less than a day, it is necessary to work on further optimization, the reserves are existing.

If the conditions on the grid parameters are not fulfilled (first of all, if the time step is too large), a numerical instability arises near the photospheric boundary, as a result of which an unnaturally large disturbance propagates into the corona (Fig. 1). However, if, after the onset of instability, we return to the selected parameters of the difference scheme, then the instability stabilizes and the strong disturbance in the corona caused by the instability will disappear. This indicates the quality of the proposed difference scheme.

\section{Stabilization of the instability arising at the non-photospheric boundary}

Calculations in the real scale of time have shown appearance of sufficiently strong disturbances caused by instabilities at the non-photospheric boundary. Such instabilities (Fig. 
2) can lead to a halt in the calculation due to a strong increase in the values or to an obviously incorrect solution due to the appearance of strong nonphysical perturbations.

This problem was solved by using the following methods:

1. Limiting the velocity of plasma inflow into the computational domain.

2. Application of artificial viscosity (usual and magnetic) near the non-photospheric boundary.

3. The invariability of the magnetic field at the edges of the boundary of the computational domain, relative to the potential field used to set the boundary conditions.
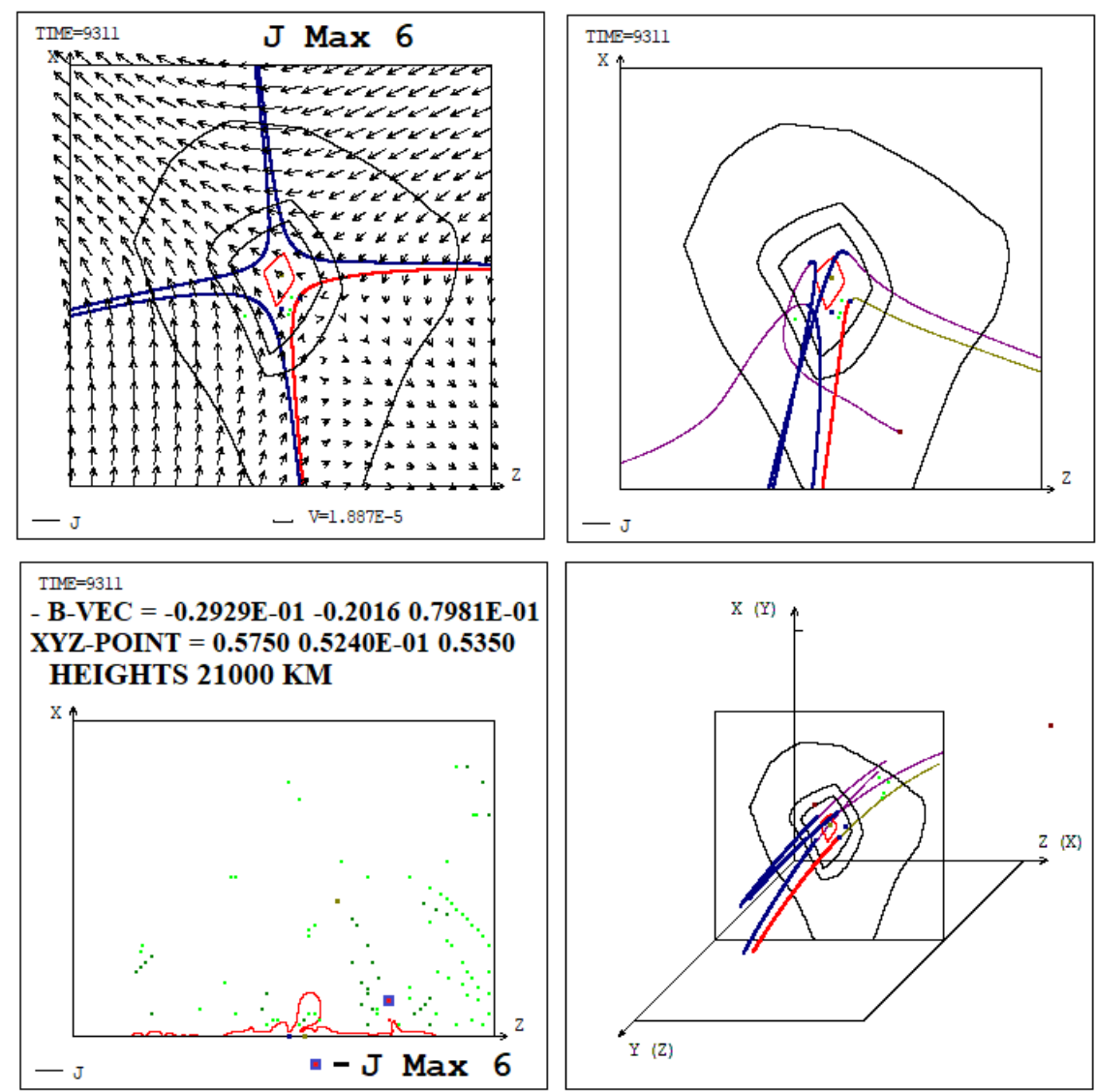

Fig. 4. Magnetic field configuration and plasma flow near the 6-th maximum of current density.

Formation of singular $X$-type lines with the plasma flow, contributing to the accumulation of flare energy.

Magnetic field configuration in corona obtained by MHD simulation in the real scale of time is shown in Fig 3. During the evolution of magnetic field and plasma, described by the results of MHD simulation in the real scale of time, from time to time the current density maximums appear with X-type configuration and plasma flow as for 6-th maximum at the moment 0.658 days presented in Fig. 4. The plasma flow near such maximums in principle can cause to the current sheet creation. Some of such configurations later disappear, possible, the microflares occur in such configurations.

\section{Conclusion}

1. Solar flares occur as a result of the release of magnetic energy stored in the magnetic field of the current sheet of the solar corona. To study the physical mechanism of the flare and improve the quality of the flares prognosis, it is necessary to carry out MHD simulation in the corona above the AR in real scale of time. 
2. Parallelization of the calculation for solving a specially developed finite-difference scheme of MHD equations is carried out. The optimal parameters of the difference scheme were selected for calculations in the real scale of time.

3. The methods have been developed to stabilize the numerical instability arising at the non-photospheric boundary.

4. When the selected parameters of the difference scheme change (first of all, an increase in the time step), numerical instability can appear at the photospheric boundary, causing to the propagation of a strong nonphysical disturbance into the computational domain of the corona. When returning to the selected optimal parameters of the difference scheme without performing a new calculation from an earlier time instant before the onset of instability, the instability stabilizes, and the disturbance propagating from it into the corona disappears, which confirms the quality of the developed difference scheme.

5. The optimization of the parallelization algorithm has been carried out, first of all, the data exchange (values in the computational domain of the corona) between the main memory of the computer and the memory of the graphics card on which the computation is parallelized is minimized. As a result of the optimization performed, the calculation speed increased 7.5 times.

6. The calculation of the evolution of the plasma and the field in the corona above AR 10365 at the initial stage did not show the appearance of pronounced current sheets even with sufficiently strong disturbances on the photosphere (exceeding the real disturbances that appeared due to numerical instabilities in the process of choosing the optimal parameters of the difference scheme). At several current density maxima, an X-type configuration was found, with a plasma flow, which should cause to the formation of a current sheet; however, in the course of further evolution, such configurations disappeared (possibly, microflares appeared).

7. The work performed has shown the possibility of further optimization of the methods in order to further reduce the calculation time.

\section{References}

Borisenko A.V., Podgorny I.M., Podgorny A. I. (2020). Using of parallel computing on GPUs for MHD modeling of solar flare behavior in real time scale. Proc. 12-th Workshop "Solar Influences on the Magnetosphere, Ionosphere and Atmosphere", Primorsko, Bulgaria, June, 2020. This Issue.

Lin R.P., Krucker S., Hurford G.J. et al. (2003) RHESSI observations of particles acceleration and energy release in an intense gamma-ray line flare. Astrophys. J., 595, L69-L76.

Podgorny I.M., Dubinin E.M., Israilevich P.L., Nicolaeva N.S. (1988) Large-scale structure of the electric field and field-aligned currents in the auroral oval from the Intercosmos-Bulgaria satellite data. GRL. 15, 1538-1540.

Podgorny A.I., Podgorny I.M. (2004) MHD Simulation of Phenomena in the Solar Corona by Using an Absolutely Implicit Scheme. Computational Mathematics and Mathematical Physics. 44, 1784-1806.

Podgorny A.I., I.M. Podgorny I.M. (2008) Formation of Several Current Sheets Preceding a Series of Flares above the Active Region AR 0365. Astronomy Reports. 52, 666-675.

Podgorny I.M., Balabin Yu.V., Vashenuk E.M., Podgorny A.I. (2010) The generation of hard X-rays and relativistic protons observed during solar flares. Astronomy Reports. 54, 645-656.

Podgorny A.I., Podgorny I.M., Meshalkina N.S., (2015) Dynamics of Magnetic Fields of Active Regions in Preflare States and during Solar Flares. Astronomy Reports. 59, 795-805.

Podgorny I.M., A.I. Podgorny A.I. (2018) Diagnostic of a Solar Flare via Analyses of Emission in Spectral Lines of Highly Ionized Iron. Astronomy Reports. 62, 696-704.

Syrovatskii, S.I. (1966) Dynamic Dissipation of Energy in the Vicinity of the Magnetic Field Neutral Line. Zh. Eksp. Teor. Fiz. 50, 1133-1147. 


\title{
A Comparison of the Neutron Dose Measurements Completed by the Bulgarian and Russian Instruments Outside the Zvezda Module of the ISS
}

\author{
Dachev $^{1}$ T., Semkoval J., Tomov ${ }^{1}$ B., Matviichuk ${ }^{1} Y$., Dimitrov ${ }^{1} P .$, Koleva $^{1}$ R., \\ Jordanova ${ }^{1}$ M., Bankov ${ }^{1}$ N., Litvak ${ }^{2}$ M., Mitrofanov ${ }^{2}$ I., Golovin ${ }^{2}$ D., Kozyrev ${ }^{2}$ A., \\ Malakhov ${ }^{2}$ A., Mokrousov ${ }^{2}$ M., Sanin ${ }^{2}$ A., Tretyakov ${ }^{2}$ V., \\ Fedosov ${ }^{2}$ F., Ploc ${ }^{3}$ O., Shurshakov ${ }^{4}$ V., Benghin ${ }^{4}$ V.
}

${ }^{1}$ Space Research and Technology Institute, Bulgarian Academy of Sciences, Sofia, Bulgaria

E-mail: tdachev@bas.bg

${ }^{2}$ Space Research Institute, Russian Academy of Sciences, Moscow, Russia

${ }^{3}$ Nuclear Physics Institute, Czech Academy of Sciences, Prague, Czech Republic

${ }^{4}$ State Research Center Institute of Biomedical Problems, Russian Academy of Science, Moscow, Russia

\begin{abstract}
The aim of this article is to compare the neutron dose measurements completed by the Bulgarian and Russian instruments outside the Zvezda module of the International Space Station (ISS). The data from two instruments are used. The Bulgarian Liulin type spectrometer R3DR [Dachev et al., 2015] and the Russian BTN device [Litvak et al. 2017]. Both instruments were mounted outside the Zvezda module of the ISS.

The first part of the paper describes both instruments and illustrates that they are situated at a 4-5 meters apart from each other. A detailed description of the procedure for the evaluation of the ambient dose equivalent $\left(H^{*}(10)\right)$ is presented.

The assessment of the equivalent dose rates of neutron radiation, measured by BTN and R3DR instruments, reveals that R3DR and BTN values are comparable in both equatorial and South Atlantic Anomaly (SAA) regions, while the R3DR values are higher that the BTN values in the high latitude regions.
\end{abstract}

\section{Introduction}

Neutrons at the height of the board the International Space Station (ISS) are produced in three physical processes [Tret'yakov et al., 2010]. First, energetic protons and ions from the galactic cosmic rays and from the radiation belts of the Earth's magnetosphere generate neutrons with energies of about 10-20 MeV due to the nuclear reactions in the upper atmosphere of the Earth. The produced secondary neutrons are slowed in the atmosphere matter. Part of them are absorbed in the nuclear reactions of capture to form new nuclei. Another part is decayed, but a substantial fraction leaves the atmosphere [Nymmik, 1993], forming in the near-terrestrial space the so-called neutron albedo of the Earth.

Second, around the ISS the proper induced neutron emission is produced due to the interactions of the energetic charged particles of the magnetosphere with the matter of the station itself [Sevast'yanov et al., 1997]. These local neutrons fluxes are present around the station and they are variable along the station orbit due to irregularity of the flux of charged particles.

The third cause of the existence of neutrons in the near-terrestrial space is the solar activity. It is well known that during some powerful solar energetic particles (SEPs) fluxes of high-energy neutrons are generated [Share et al., 2007], [Xiao et al. 2016] reports a total of 12 solar events in which neutrons even reached the Earth ground between 1982 and 2015.

\section{Instrumentation}

\section{BTN instruments description}


The BTN-MD detection block (Fig. 1) is a spectrometer with four individual neutron detectors. BTN-MD is a spare flight unit of the HEND experiment, installed onboard NASA spacecraft Mars Odyssey, intended for the exploration and cartography of neutron radiation of Mars surface in different energy ranges [Mitrofanov et al., 2002]. After several years of successful operation of HEND, it was decided to use a spare flight unit for the near Earth measurements onboard the ISS [Tret'yakov et al., 2010]. The Russian Zvezda module was chosen for this. Some preparations were made, such as development of the BTN-ME inner control block, the Block-MF (a system for providing temperature conditions) and a special mounting pylon for the BTN-MD outside the station [Tret'yakov et al., 2010].

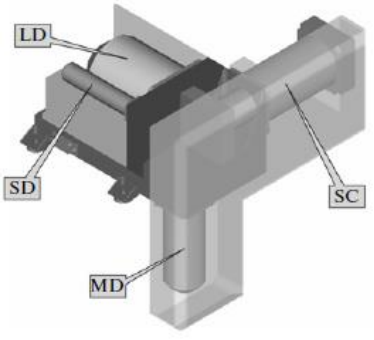

Fig. 1. Structure chart of the BTN-MD unit.

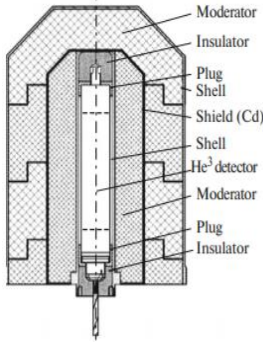

Fig. 2 Design features of $B T N-M D$ instrument

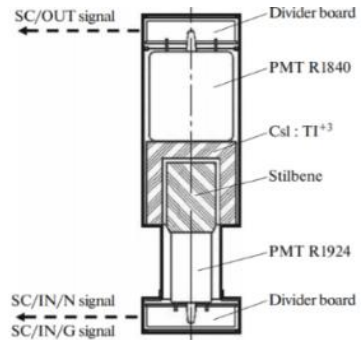

Fig. 3. Scintillation block configuration.

Three detectors (SD, MD, LD (pls. see Fig. 2.) are based on helium-filled $\left({ }^{3} \mathrm{He}\right.$ ) proportional neutron counters, covered in cadmium shields and polyethylene spheres of different thickness [Litvak et al. 2017]. The proportional ${ }^{3} \mathrm{He}$ counters are detectors of epithermal neutrons. In this case, having given a good account (also as a part of the HEND experiment flight unit), the LND2517 industrial counters (LND Inc. Company, United States) were used, filled with ${ }^{3} \mathrm{He}$ and a small addition of $\mathrm{CO}_{2}$ under an atmosphere pressure of 6 . The registration concept in the proportional counter is based on the capture of a thermal or epithermal neutron in a reaction of that type as follows:

$$
\mathrm{n}+{ }^{3} \mathrm{He} \rightarrow{ }^{3} \mathrm{H}+\mathrm{p}+764 \mathrm{keV}
$$

The total energy output in that reaction is distributed between the proton and the triton in the ratio of $191 \mathrm{keV}$ (triton) and $573 \mathrm{keV}$ (proton) in inverse proportion to their masses.

The counter itself is a metallic cylinder (cylinder jacket serves as a cathode) with a thin (thickness about the fraction of a millimeter) tungsten wire along the cylinder axis, which serves as the anode. A current of about $1 \mathrm{kV}$ is applied between the cathode and the anode. This allows the counter to work in the socalled proportional mode, when the applied current is high enough for the primary electrons formed during the ionization of gas molecules by a nuclear reaction of $n+{ }^{3} \mathrm{He} \rightarrow{ }^{3} \mathrm{H}+\mathrm{p}$ in order to perform a secondary ionization with the generation of a large number of ion pairs. The collected charge on the electrodes of the counter and the corresponding current impulse is proportional to the full energy output in the volume of the counter. The digitalization of the signal received, during one neutron registration act, allows the amplitude spectrum to be obtained.

The fourth detector (SC (pls. see Fig. 3.) is intended to register only high-energy neutrons in the energy range of $350 \mathrm{keV}$ to $15 \mathrm{MeV}$. It was manufactured from a stilbene crystal (C14H12) and covered with the anticoincidence shield from the crystal.

Figure 4 shows the sensitivity functions of all detectors in the BTN-MD instrument. It is clearly seen that, due to various thicknesses of the moderators, in the proportional counters, we managed to succeed in registering neutrons in a wide range of energies of from $0.4 \mathrm{eV}$ up to 15 $\mathrm{MeV}$.

\section{R3DR instruments description}

The EXPOSE-R missions on the ISS was performed by the European Space Agency (ESA) for astrobiological studies under space conditions as provided in low-Earth orbit outside the ISS. [Rabbow et al., 2015]. The radiation fields encountered in this environment is of pivotal interest to astrobiology [Ferrari and Szuszkiewicz, 2009]. To provide information about the 
diurnal variation of this radiation, the EXPOSE-R platform accommodated the Radiation Risk Radiometer-Dosimeter (R3D) for the Russian segment (R) R3DR (Fig. 5). It is a low mass and small-dimension automatic device measuring solar radiation in four channels and cosmic ionizing radiation in 256 channels during the EXPOSE-R mission in 2008-2009. R3DR instrument was developed and build in cooperation by Bulgarian and German scientists after a successful participation in the ESA announcements of opportunity in 1996, 2001 and 2003 [Dachev et al., 2015 and 2017].

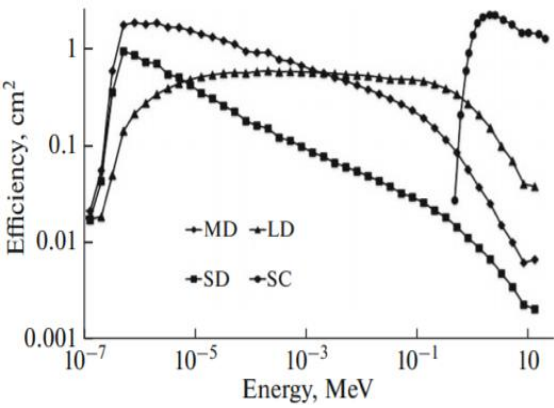

Fig. 4. Sensitivity functions for all BTN-MD instrument detectors.

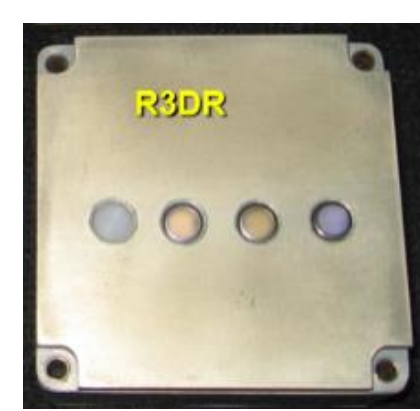

Fig. 5. R3DR instrument.

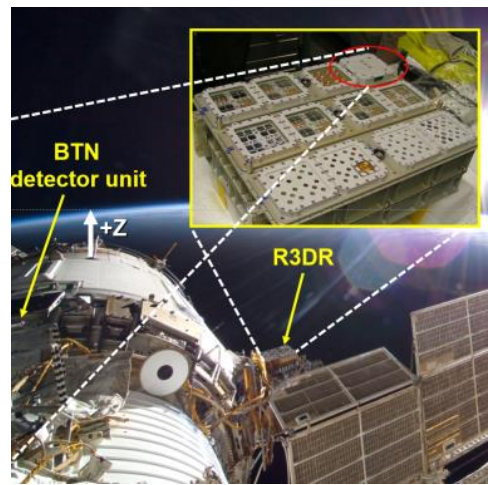

Fig. 6 Position of the BTN detector unit and R3DR instrument outside ISS Zvezda module.

The R3DR instrument is single detector Liulin type spectrometer. Its block diagram and dose calculation procedure are described in the paper by Dachev et al., named "Registration of neutrons with a single detector Liulin type spectrometer" in this issue.

Recently, Dachev et al., (2017) published a comprehensive description of the R3DR2 instrument and its radiation sources selection procedures. Therefore, we will skip those details, as R3DR unit is identical to the R3DR2.

Fig. 6 shows the mounting position of the EXPOSE-R platform with the mounted inside R3DR instrument and the BTN detector at the left side of the Russian Zvezda module of the ISS. The BTN detector unit is seen in the zenith area of the module. Both units, BTN detector and R3DR instrument, are at a distance of about 4-5 meters.

\section{Energy deposition spectra variations by source and location}

Fig. 3 of the paper by Dachev et al., named "Registration of neutrons with a single detector Liulin type spectrometer" in this issue presents the shapes of the galactic cosmic ray (GCR) spectra only.

Fig. 7 here illustrates the shapes of all three-radiation sources for the period May 1, 2010July 11, 2010. There are two spectra for the GCR, one for the high latitudes (HL) and another for the equatorial (Eq) latitudes. Two more spectra are included - one for the outer radiation belt (ORB) and one for the inner radiation belt (IRB).

Fig. 8 visualizes the geographic locations of the different areas and sources, mentioned in Fig. 7. The isolines of the L-shell values from 1 to 5 for the 2005 epoch [Mcllwain, 1961; Heynderickx et al., 1996] at the altitude of the ISS are presented with pink dashed lines in Fig. 8. The equatorial GCR region is seen with red ellipse where the effective cutoff rigidity $-R_{c}$ values are greater than $15.4 \mathrm{GV}$. This region is in the middle of the $\mathrm{L}$, equal to one isoline.

The south and north high latitude (HL) regions are plotted with blue areas. They are characterized by $R_{c}$ less than $0.85 \mathrm{GV}$ in north and $0.62 \mathrm{GV}$ in the south hemispheres respectively. These definitions of the GCR source locations was used by Litvak et al., (2017). Here we apply the same definitions to make the comparisons presented in Table 1 . $\left(R_{c}\right.$ values were calculated using the Shea et al., (1985) relations between L-shell parameter and $R_{c}$ values.) 
The IRB region is seen with light green area, where the total Earth magnetic field strength is less than 0.24 Gauss.

The light brown points show the positions where the ORB particles were observed in 2015 . The maximum at about $20^{\circ}$ East longitude with $\mathrm{L}=2.5$ gives the $\mathrm{ORB}$ position during the magnetic storms [Dachev, 2018 see Fig. 2 therein].

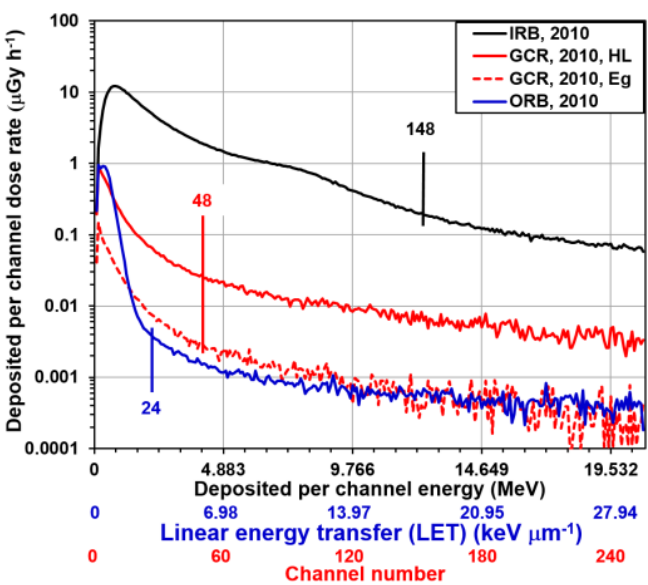

Fig. 7. A comparison of the spectra shapes by different radiation sources.

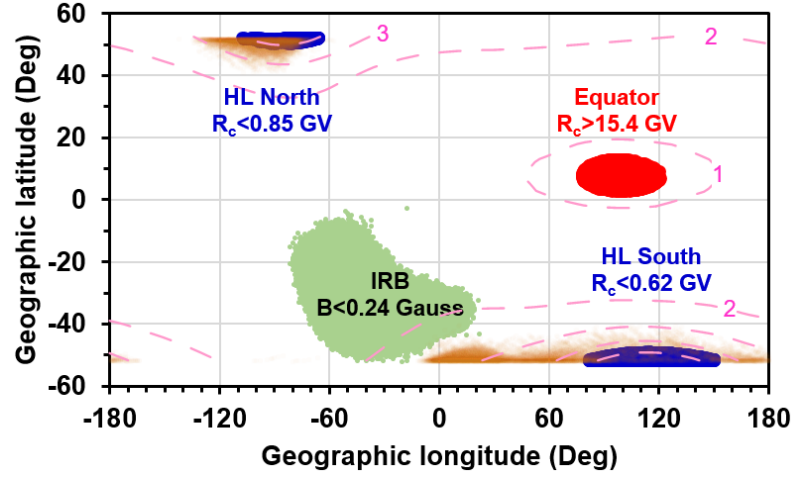

Fig. 8. The global distribution of places in Fig. 7.

In Fig., 7 the deposited per channel dose rates in $\mu \mathrm{Gy} \mathrm{h} \mathrm{h}^{-1}$ are plotted against the deposited per channel energies in MeV. For a better understanding of the Fig. 7, two additional horizontal

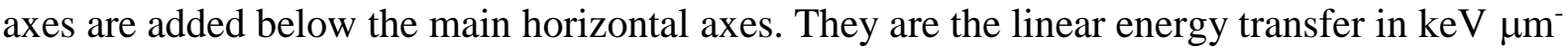
1 and the channel number.

Fig. 7 clarifies the selection procedure of the high and low LET parts of the spectra. The ORB and GCR spectra curves shapes are similar, showing a part with a high slope in the low LET energy deposition region and a smaller slope part in the high LET. We consider that the end of the low LET part of the spectra is at the point of the slope changes. There the low LET part finishes and the high LET part of the spectrum begins. From Fig. 7 it is seen that for the GCR source, the selection point is at channel 48, while for the ORB source it is at channel 24.

Fig. 7 depicts that the GCR low LET source spectra part shows a maximum up to $0.1 \mu \mathrm{Gy}$ $\mathrm{h}^{-1}$, which has to be populated by high-energy protons from a galactic origin. Above channel number 48, the spectra continues with a small slope part that contains mainly secondary neutrons protons penetrating trough the side walls of the detector and neglectable amount of $\mathrm{He}^{+}$ions.

There are two GCR spectra in Fig. 7. The higher dose rate spectrum presents the averaged dose rates in the high latitude (HL) regions, while small dose rate spectrum presents the equatorial region (Eq). The explanation is that the high latitude spectrum contains larger amount of primary GCR particles, being in smaller vertical cutoff rigidity [Smart \& Shea, 2005].

The IRB spectra in Fig. 7 is obtained inside of the inner radiation belt at a total magnetic field strength B less than 0.24 Gauss. It is populated mainly by protons in the energy range 10$200 \mathrm{MeV}$ [Nealy et al., 2007]. The two main peculiarities seen in the shape of this spectrum are:

(a) The maximum in the deposited dose is close to $0.9 \mathrm{MeV}$ deposited energy. It is generated by the SAA mean energy protons of about $40 \mathrm{MeV}$ [Dachev et al., 2017a];

(b) There is a knee at about $9.1 \mathrm{MeV}$ deposited energy.

Usually the change of the slope of the IRB spectrum appears close to $6 \mathrm{MeV}$ deposited energy at channel 78. This corresponds to the stopping energy of the normally falling protons to the detector with $0.3 \mathrm{~mm}$ thickness [Dachev, 2009]. The exact value for $0.3 \mathrm{~mm}$ silicon is 6.2 $\mathrm{MeV}$ as seen in the tables provided by Berger et al. [2020] at 
http://physics.nist.gov/PhysRefData/Star/Text/contents.html. Similar result was obtained during the calibrations of Liulin 4 type instrument with $7.8 \mathrm{MeV}$ incident energy protons, falling normally to the detector (Fig. 4 of Dachev et al., 2017a). 6.2 MeV deposited energy is registered in the channel 78. This means that all other channels up to 256 of the spectrometer are populated by long path length low LET protons or by heavier ions. In the specific case, the R3DR spectrum is moved to the right and is registered in channel 93. The reason is the place where R3DR spectrometer is located (Fig. 6). The latter is mounted very close to the body of Zvezda module, which partially shielded the SAA protons, drifting almost horizontally from West to East. In order to keep the same requirements for the selection procedure of the high and low LET parts of the spectrum, we decided to choose as a point of change of the slope of the IRB spectrum to channel 148.

The ORB spectrum (Fig. 7) shows huge maximum up to $1.5 \mu \mathrm{Gy} \mathrm{h} \mathrm{h}^{-1}$ in the channel numbers 0 to 24 . This part of the spectrum is populated by relativistic electrons, trapped in the ORB. The spectrum slope, registered between channels 24 and 256, is very similar to the GCR spectrum slope. This is reasonable because the relativistic ORB electrons are observed at high latitude regions (please look Fig. 8), where the GCR particles are the main permanently detected source.

\section{Evaluation of the ambient dose equivalent $(H *(10))$ with SDLTS}

Bartlett et al., [2006] wrote, "Secondary neutrons generated from the primary galactic cosmic radiation and trapped particles can be a major contributor to dose equivalent and effective dose on a spacecraft and ISS. The amount of shielding of the primary radiation manage the exact proportion, which lie in the range of $10-60 \%$."

Benton et al., [2001] wrote, "Dose equivalent rate measurements of neutrons with energies $>1 \mathrm{MeV}$ ranged from $4.5 \mu \mathrm{Sv} \mathrm{d}^{-1}$ on the low altitude STS-3 mission to $172 \mu \mathrm{Sv} \mathrm{d}^{-1}$ on the $\sim 6$ year LDEF mission. Thermal neutrons $(<0.3 \mathrm{eV})$ were observed to make a negligible contribution to neutron dose equivalent in all cases. The major fraction of neutron dose equivalent was found to be from neutrons $>1 \mathrm{MeV}$ and, on LDEF, neutrons $>1 \mathrm{MeV}$ are responsible for over $98 \%$ of the total neutron dose equivalent. Estimates of the neutron contribution to the total dose equivalent are somewhat lower than model estimates, ranging from $5.7 \%$ at a location under low shielding on LDEF to $18.4 \%$ on the highly inclined $\left(82.3^{\circ}\right)$ Biocosmos-2044 mission". https://doi.org/10.1016/S1350-4487(01)00047-6

Our assumption is that the R3DR instrument has the sensitivity to detect neutrons is based on the:

(1) Calibration results, presented in the paper Dachev et al., "Registration of neutrons with a single detector Liulin type spectrometer" in this issue.

(2) The simulations by the computer codes FLUCA, GEANT4 and PHITS, performed by Luszik-Bhadra et al. [2010] to investigate the "Response calculations for silicon-based directreading dosimeters for use at the International Space Station". This paper has a section, which uses the LULIN type geometry. It estimates the expected dose equivalent. The highest effective dose equivalent is obtained from protons $\left(178 \mu \mathrm{Sv} \mathrm{d} \mathrm{d}^{-1}\right)$, followed by Fe ions $\left(44 \mu \mathrm{Sv} \mathrm{d} \mathrm{d}^{-1}\right)$, neutrons $\left(23 \mu \mathrm{Sv} \mathrm{d}^{-1}\right)$ and He ions $\left(13 \mu \mathrm{Sv} \mathrm{d}{ }^{-1}\right)$. The mean dose equivalent as calculated for the DOS-2005 and the LIULIN devices agrees with the calculated values of effective dose equivalent better than a factor of two.

(3) The calibrations performed in CERN by Zhang et al., [2011] to investigate the fast neutron induced nuclear counter effect in Hamamatsu S2744-08 silicon PIN diodes that are the same as the diodes used by us in the R3DR instrument.

According to [Benton et al., 2001] and the results shown in Fig. 4 of [Dachev et al., Registration of neutrons with ..., this issue], we expect that we may detect by the single detector Liulin type spectrometers (SDLTS) neutrons with energies between 1 and $50 \mathrm{MeV}$. 
Historically, two attempts for the calculation of the SDLTS ambient dose equivalent $\left(H^{*}(10)\right)$ at spacecraft altitudes were developed. Both are based on the similarity of the neutron spectra at aircraft and spacecraft altitudes [Bartlett et al., 2006], http://wrmiss.org/workshops/tenth/pdf/ex01_bartlett.pdf (pls. see slide No 11 therein).

We calculate the ambient dose equivalents from the measurements using the quality factor (Q) as defined in the [ICRP Report No. 60].

In the first attempt [Spurny et al., 2006, 2007, 2009] $H^{*}(10)$ is calculated from the deposited energy spectra, supposing that events with deposited energy less than $1 \mathrm{MeV}$ correspond to low LET radiation and events with deposited energy higher than $1 \mathrm{MeV}$ correspond to the sum of the neutron and heavier charged particles (HECP) of the GCR. The data were treated in the following way:

a) For the low LET component $D(\mathrm{Si}) \rightarrow D$ (tissue) $\rightarrow H^{*}(10)$;

b) For the neutron contribution in the region of $E \mathrm{dep}>1 \mathrm{MeV}$. This is the same procedure as on board aircraft [Spurny and Dachev, 2002] that was adopted, considering that the neutron spectra are in both cases similar [Bartlett et al., 2006, pls. see slide No 9 therein]. The supposed average quality factor for this component of galactic cosmic radiation is equal to about 5;

c) For the HECP $H^{*}(10)$ is obtained as: $D(\mathrm{Si}) \rightarrow D($ tissue); $5 \times D$ (tissue $)=H^{*}(10)$; i.e., supposing that the average quality factor for this component of the galactic cosmic radiation is equal to about 5 ;

d) For the flights inside the IRB, is supposed that all events are due to protons with average $\mathrm{QF} \sim 1.3$, calculated from the energy deposition spectra in Si detector of MDU-Liulin. Finally, the following equations were used:

$$
\begin{aligned}
& H^{*}(10)=Q_{1} * D_{\text {Low LET }}+Q_{2} * D_{\text {High LET }} \\
& H_{\mathrm{GCC}}{ }^{*}(10)=1 * \sum_{1}^{14}(\mathrm{Ei} * \mathrm{Ai}) / \mathrm{MD}+5 * \sum_{15}^{256}(\mathrm{Ei} * \mathrm{Ai}) / \mathrm{MD} \\
& H_{I R B}{ }^{*}(10)=1.3 * \sum_{1}^{14}(\mathrm{Ei} * \mathrm{Ai}) / \mathrm{MD}+1.3 * \sum_{15}^{256}(E i * A i) / M D
\end{aligned}
$$

In the above preliminary equations, the deposited energy in channel number 14 is equal to 1 $\mathrm{MeV}$ deposited energy. Equation number (4) was used for a calculation of the ambient dose equivalent $\left(H^{*}(10)\right)$ at aircraft altitudes.

The equations above were used for a comparison of the calculated $H^{*}(10)$, obtained with Liulin-E094 in 2001 and R3DE instruments in 2008 with NASA TEPC at the ISS (https://srag.jsc.nasa.gov/SpaceRadiation/How/How.cfm). The obtained satisfactory results were published in 2010 [Dachev et al., 2010, http://wrmiss.org/workshops/fifteenth/Dachev.pdf].

Fig. 9 shows in details the comparison of absorbed and equivalent doses obtained by R3DE instrument and NASA TEPC in 2008.

The second attempt for the calculation of the SDLTS and particularly of the R3DR ambient dose equivalents $\left(H^{*}(10)\right.$ from neutrons is described in the next paragraphs of this paper, i.e.:

It is assumed that the end of the low LET part of the deposited energy spectra for the different sources of radiation as GCR, IRB and ORB is at different channels numbers, i.e. 48 for the GCR, 148 channel for the IRB and 24 for the ORB. This is well illustrated in Fig. 7 as well as on Fig. 5d and Fig. 6a in the paper by Dachev et al. "Registration of neutrons with a single detector Liulin type spectrometer" in this issue.

The first attempt for the calculation of the ambient dose equivalents $\left(H^{*}(10)\right.$ assumes that the deposited energy in channel number 14 (equal to $1 \mathrm{MeV}$ deposited energy) is the last channel of the low LET part of the deposited energy. It is fixed for all radiation sources.

As our goal is the comparison of the neutron dose equivalents, measured with the BTN instrument, only the high LET part of the following equations are used.

$$
\begin{aligned}
& H_{G C R}{ }^{*}(10)=3.16 * \sum_{48}^{256}(\mathrm{Ei} * \mathrm{Ai}) / \mathrm{MD} \\
& H_{I R B \text { and } S E P}{ }^{*}(10)=1.7 * \sum_{148}^{256}(\mathrm{Ei} * \mathrm{Ai}) / \mathrm{MD}
\end{aligned}
$$




$$
H_{O R B}{ }^{*}(10)=1 * \sum_{24}^{256}(\mathrm{Ei} * \mathrm{Ai}) / \mathrm{MD}
$$

The conversion quality factors for GCR $(\mathrm{Q}=3.16)$ and IRB $(\mathrm{Q}=1.7)$ sources in the equations (6) and (7) was obtained by averaging of 923,114 measurements in the CGR source and 60,973 in the "Trapped" source performed by the NASA TEPC in the period from Mach 1, 2008 till March 31 ,

[https://www.nasa.gov/mission_pages/station/science/experiments/TEPC.html]. The TEPC data were provided by [Zapp, 2013] and by the Coordinated Data Analysis Web' at the Goddard Space Flight Center [http://cdaweb.gsfc.nasa.gov/].

Luszik-Bhadra et al. [2010] in Fig 2 therein limited the list of the heavier ions to Helium and Iron. Iron ions are out of the range of the SDLTS [Uchihori et al. 2002]. The amount of $\mathrm{He}^{+}$ ions in the spectral fluence rate in the cited above Fig. 2 of Luszik-Bhadra et al. [2010] in the energy range 10-100 $\mathrm{MeV}$ are two orders of magnitude below that of the neutrons fluence rate

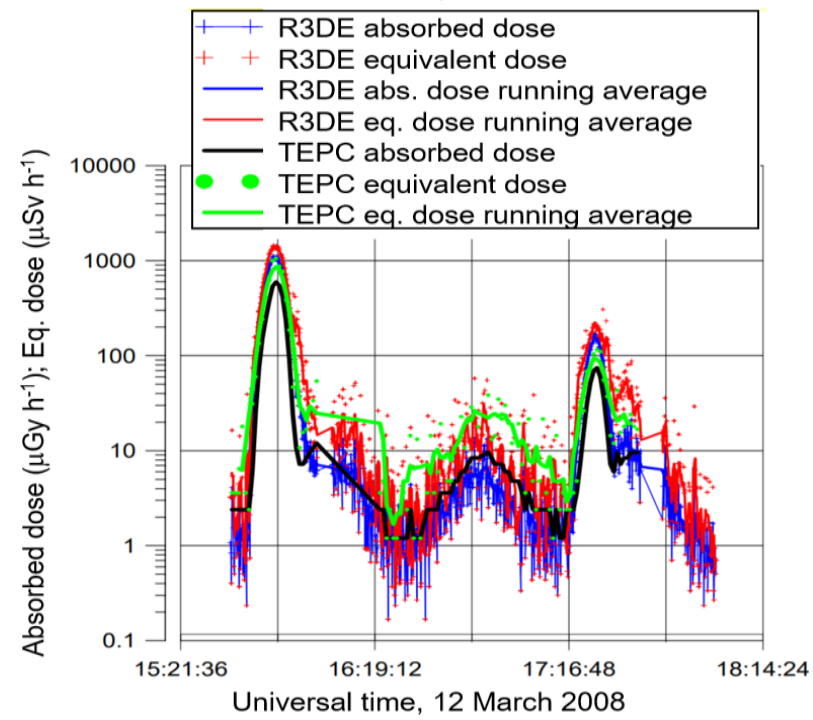

Fig. 9. A comparison of absorbed and equivalent doses obtained by R3DE instrument and NASA TEPC.

outside the ISS. That is why; we decided to neglect this very low fluence rate of $\mathrm{He}^{+}$ions and to consider that the completely high LET parts of the GCR, IRB and ORB spectra are populated only by neutrons.

\section{Comparisons of the averaged neutron equivalent dose rates}

The equations 6-8, described in the previous section, were used to calculate the equivalent dose rates measured by R3DR instrument in the period May 1, 2010- July 11, 2010.

Table 1 presents the results from the comparison of the equivalent dose measurements at various segments of the orbit. Three experiments are listed: BBND inside the ISS [Koga et al., 2011], BTN outside the ISS [Tret'yakov et al., 2010], [Litvak et al. 2017] and R3DR outside the ISS [Dachev et al., 2015].

Table 1 .

\begin{tabular}{|c|c|c|c|c|c|}
\hline \multirow[t]{3}{*}{$\begin{array}{c}\text { Experiment, } \\
\text { Dates of measurements, } \\
\text { Solar activity level }\end{array}$} & \multirow[t]{3}{*}{$\begin{array}{c}\text { Measurement condit., } \\
\text { Detectors, } \\
\text { Range of measurements }\end{array}$} & \multicolumn{4}{|c|}{$\begin{array}{l}\text { Estimation of the average neutron equivalent dose } \\
\text { rate accumulation at various segments of the orbit, } \\
\qquad S v h^{-1}\end{array}$} \\
\hline & & \multicolumn{2}{|c|}{ GCR } & \multirow{2}{*}{ IRB (SAA) } & \multirow{2}{*}{ ORB } \\
\hline & & Equator & High lat. (HL) & & \\
\hline $\begin{array}{c}\text { BBND [Koshishi et al., } \\
\text { 2007], } \\
\text { Mar 23, 2001-Nov 15, } \\
2001, \\
\text { Max. of } 23 \text { rd cycle }\end{array}$ & $\begin{array}{c}\text { Inside the ISS, } 6 \text { sensors } \\
\text { 3He and one scintillation } \\
\text { detector, } \\
0.03 \mathrm{eV}-15.0 \mathrm{MeV}\end{array}$ & $0.3-1$ & $2-6$ & $10-100$ & \\
\hline
\end{tabular}




\begin{tabular}{|c|c|c|c|c|c|}
\hline $\begin{array}{c}\text { BTN, [Tret'yakov et al., } \\
\text { 2010], } \\
\text { Feb 26-present time, } \\
\text { A half of the solar activity } \\
\text { cycle }\end{array}$ & $\begin{array}{c}\text { Outside the ISS } \\
3 \text { sensors }{ }^{3} \mathrm{He} \text { and one } \\
\text { scintillation detector, } \\
0.4 \mathrm{eV}-10.0 \mathrm{MeV}\end{array}$ & 0.2 & 0.8 & 5 & \\
\hline $\begin{array}{c}\text { BTN, [Litvak et al., 2017], } \\
\text { Feb 26-present time, } \\
\text { A half of the solar activity } \\
\text { cycle }\end{array}$ & $\begin{array}{c}\text { Outside the ISS } \\
3 \text { sensors }{ }^{3} \mathrm{He} \text { and one } \\
\text { scintillation detector, } \\
0.4 \mathrm{eV}-10.0 \mathrm{MeV}\end{array}$ & $0.1-0.4$ & $1-5$ & $>50$ & \\
\hline $\begin{array}{c}\text { R3DR, [Dachev et al., 2015] } \\
\text { May 1, 2010- July } 11,2010 \\
\text { Minimum of the solar } \\
\text { activity cycle }\end{array}$ & $\begin{array}{c}\text { Outside the ISS, } \\
\text { One PIN diode detector, } \\
1-50 \mathrm{MeV}\end{array}$ & 0.43 & 6.3 & 21 & 4 \\
\hline
\end{tabular}

Table 1 reveals that the both the BBND and R3DR average equivalent dose rates are similar in the high latitudes and the SAA region. Close to the equator, the BBND dose values [Koga et al., 2011] are about 8 times higher than the R3DR values.

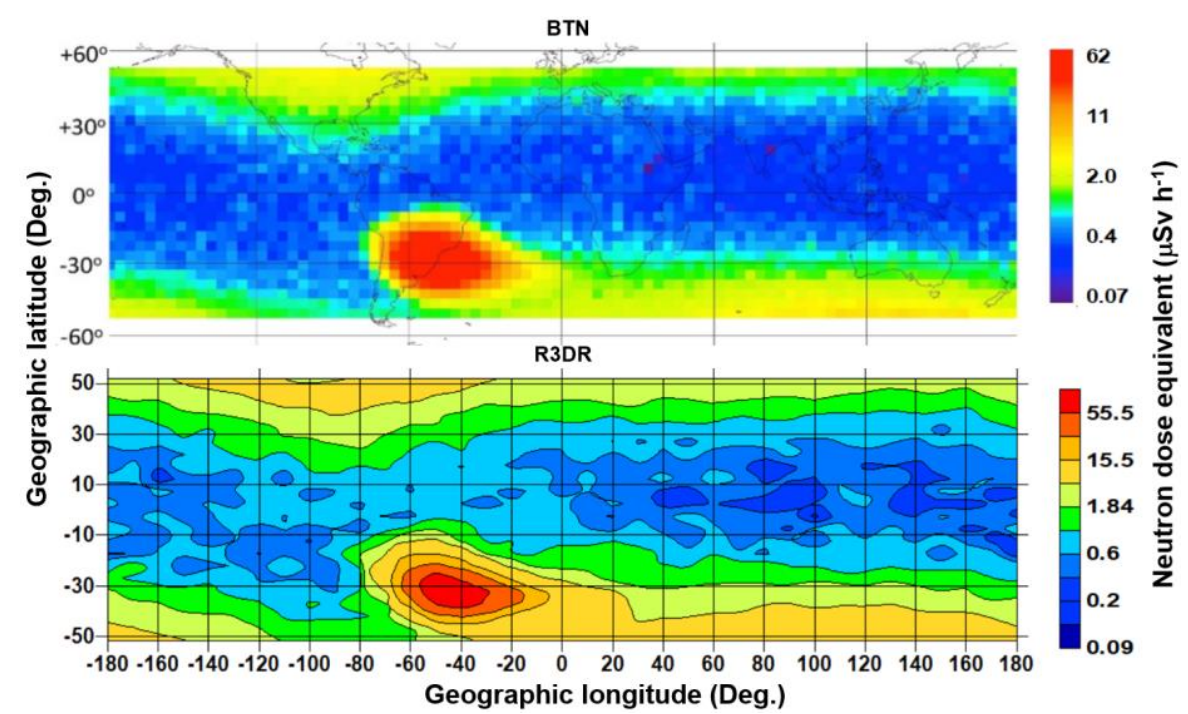

Fig. 10. Equivalent dose rate of neutron radiation measured by BTN and R3DR spectrometer.

The comparison of the equivalent dose rate values obtained with R3DR instrument and BTN shows the following:

a) The BTN values close to the equator are comparable with the R3DR values;

b). The probable reason is that the energy range of the measured neutrons of the R3DR instrument is from 1 to $50 \mathrm{MeV}$, while the BTN range is up to $16 \mathrm{MeV}$.

c) In the SAA region the average equivalent dose rates, registered with BTN and R3DR are comparable.

d) Only R3DR has measured the average equivalent dose rates in the ORB region.

Fig. 10 illustrates the comparison of the global distribution of the equivalent dose rate of neutron radiation measured by BTN and R3DR spectrometer. At first glance, both distributions are quite similar. The distributions demonstrate the dependence from the Earth magnetic field and follow the global distribution of the L-values.

Fig. 10 confirms the results listed in Table 1, i.e.: R3DR values are higher that the BTN values in the high latitudes; R3DR and BTN values are comparable in both equatorial and SAA regions.

\section{Conclusions}

This paper compares the neutron dose measurements, completed by the Bulgarian R3DR spectrometer and Russian BTN instrument. They both were mounted outside the Zvezda module of the International Space Station. 
The assessment of the equivalent dose rates of neutron radiation, measured by BTN and R3DR instruments, reveals that R3DR and BTN values are comparable in both equatorial and South Atlantic Anomaly (SAA) regions, while the R3DR values are higher that the BTN values in the high latitude regions.

\section{Acknowledgements}

This work is supported by: Agreement between RAS and BAS on "Fundamental space research", Contract No. 4000117692/16/NL/NDe Funded by the Government of Bulgaria through an ESA Contract under the PECS (Plan for European Cooperating States), Contract with National Science Fund -Bulgaria on Project N 129 FOR BILATERAL PROJECTS - BULGARIA - RUSSIA 2018 -2019. In IKI-RAS the work is supported by the Russian Science Foundation under grant 19-52-18009. NPI work was funded by the European Structural and Investment Funds under the Operational Program Research, Development and Education (project CRREAT CZ.02.1.01/0.0/0.0/15_003/0000481).

Most of the data used in this paper are part of the "Unified web-based database with Liulintype instruments", which are available online, free of charge at the following URL: http://esapro.space.bas.bg/database. The database was created under the ESA contract mentioned above.

\section{References}

Bartlett D.T. and Goldhagen P. (2005), private communication of Prof. Spurny.

Benton, E. R., \& Benton, E. V. (2001), Space radiation dosimetry in low-Earth orbit and beyond. Nuclear Instruments and Methods in Physics Research B, 184(1-2), 255-294.

Dachev, Ts.P., (2009), Characterization of near Earth radiation environment by Liulin type instruments, Adv. Space Res., 44, 1441-1449. http://dx.doi.org/10.1016/j.asr.2009.08.007

Dachev, T.P., J.V. Semkova, B.T. Tomov, Yu.N. Matviichuk, Pl.G. S. Maltchev, R. Koleva, Pl., Dimitrov, N.G. Bankov, V.V., Shurshakov, V.V., Benghin, E.N., Yarmanova, O.A. Ivanova, D.-P. Häder, M.T. Schuster, G. Reitz, G. Horneck, Y. Uchihori, H. Kitamura, O. Ploc, J. Kubancak, I. Nikolaev, (2015), Overview of the Liulin type instruments for space radiation measurement and their scientific results, 92-114. http://dx.doi.org/10.1016/j.1ssr.2015.01.005.

Dachev, Ts., G. Horneck, D.-P. Häder, M. Schuster, and M. Lebert, (2015), EXPOSE-R cosmic radiation time profile, Journal of Astrobiology, 14, 17-25. http://dx.doi.org/10.1017/S1473550414000093.

Dachev, T. P., Bankov, N. G., Tomov, B. T., Matviichuk, Y. N., Dimitrov, P. G., Häder, D.-P. \& Horneck, G., (2017), Overview of the ISS radiation environment observed during the ESA EXPOSE-R2 mission in 20142016. Space Weather, 15, 1475-1489. https://doi.org/10.1002/2016SW001580.

Dachev, T.P., (2018). Relativistic Electron Precipitation Bands in the Outside Radiation Environment of the International Space Station, Journal of Atmospheric and Solar-Terrestrial Physics, 177, 247-256. https://doi.org/10.1016/j.jastp.2017.11.008

Ferrari, F. \& Szuszkiewicz, E. (2009). Cosmic rays: a review for astrobiologists. Astrobiology 9, 413-136.

ICRU, 2000. Nuclear Data for Neutron and Proton Radiotherapy and for Radiation Protection, Report 63. ICRU Publication, Bethesda, MD.

Koshiishi, H., Matsumoto, H., Chishiki, A., Goka, T. and Omodaka, T., (2007). Evaluation of the neutron radiation environment inside the International Space Station based on the Bonner Ball Neutron Detector experiment. Radiation measurements, 42(9), 1510-1520.

Litvak, M.L., Mitrofanov, I.G., Nuzhdin, I.O. et al., (2017), Monitoring of the time and spatial distribution of neutron-flux spectral density outside the Russian segment of the International Space Station based on data from the BTN-Neutron space experiment. Cosmic Res 55, 110-123. https://doi.org/10.1134/S001095251702006X

Luszik-Bhadra, M., Beck, P., Berger, T., Jaksic, A., Latocha, M., Rollet, S., Vuotila, M., Zechner, A. and Reitz, G., 2010. Response calculations for silicon-based direct-reading dosimeters for use at the international space station (ISS). Radiation measurements, 45(10), 1548-1552.

McIlwain, C. E., (1961). Coordinates for mapping the distribution of magnetically trapped particles. Journal of Geophysical Research, 66, 3681-3691. https://doi.org/10.1029/JZ066i011p03681.

Mitrofanov, I.G., Litvak, M.L., Kozyrev, A.S., et al., (2003). Search for water in Martian soil using global neutron mapping by the Russian HEND instrument onboard the US 2001 Mars Odyssey spacecraft, Sol. Syst. Res., vol. $37,5,366-377$.

Nealy, J.E. et al. (2007). Pre-engineering spaceflight validation of environmental models and the 2005 HZETRN simulation code. Adv. Space Res. 40, 1593-1610. doi: 10.1016/j.asr.2006.12.030.

Nymmik, R.A., (1993). A Model of Particle Fluxes and Energy Spectra of Solar Cosmic Rays, Kosm. Issled., vol. 31 , no. 6, pp. 51-59. 
Rabbow, E., Rettberg, P., Barczyk, S., Bohmeier, M., Parpart, A., Panitz, C., ... Reitz, G. (2015). The astrobiological mission EXPOSE-R on board of the International Space Station. International Journal of Astrobiology, 14, 3-16. https://doi.org/10.1017/S1473550414000202

Shavrin, P.I., Kuzhevskij, B.M., Kuznetsov, S.N., et al., (2002). Measurements of neutron fluxes with energies from thermal to several MeV in near-Earth space: SINP results, Radiat. Meas., 35, 5, 531-538.

Sevast'yanov, V.D., Tarnovskii, G.B., and Lyagushin, V.I., (1997). Measurement of the Neutron Energy Spectrum on the Mir Orbital Station, Kosm. Issled., 35, 2, 216-220. [Cosmic Research, pp. 201-205].

Spurný, F., (2005). Response of a Si-diode-based device to fast neutrons, Radiation Measurements, 39, 219 - 223. Spurný, F., Ploc O., Dachev, T., (2007). On the Neutron Contribution to the Exposure Level onboard Space Vehicles, Radiation Prot. Dosimetry, 126, 519 - 523. http://rpd.oxfordjournals.org/content/126/1-4/519

Spurný, F., Ploc, O., \& Jadrníčková, I., (2009). Spectrometry of linear energy transfer and dosimetry measurements onboard spacecraft and aircrafts. Physics of Particles and Nuclei Letters, 6(1), 70-77.Tret'yakov, V.I., Mitrofanov, I.G., Bobronitskii, Yu.I., et al., (2010). The first stage of the "BTN-Neutron" space experiment onboard the Russian segment of the International Space Station, Cosmic Res., 48, 4, 285-299.

Xiao, X.Y.; Hong, L.; Guan, T.Ch.; Xin, Q.L.; Jian, K.S.; Cheng, M.T., 2016. Detection of solar neutron events and their theoretical approach, New Astronomy, 39, 2

Zapp, N., (2013). SRAG group at NASA GSFC, by 'Coordinated data analysis web' at Goddard Space Flight Center, http://cdaweb.gsfc.nasa.gov/.

Zhang, L., Mao, R. and Zhu, R.Y., 2011. Fast neutron induced nuclear counter effect in Hamamatsu silicon PIN diodes and APDs. IEEE Transactions on Nuclear Science, 58(3), pp.1249-1256. 


\title{
Registration of Neutrons with a Single Detector Liulin Type Spectrometer
}

\author{
Dachev $^{l}$ T., Tomov ${ }^{l}$ B., Matviichuk ${ }^{l}$ Y., Dimitrov ${ }^{l}$ P., Semkova ${ }^{1}$ J., Jordanova ${ }^{1}$ M., Bankov ${ }^{1}$ \\ N., Ploc ${ }^{2}$ O., Litvak ${ }^{3}$ M., Tretyakov ${ }^{3}$ V., Shurshakov ${ }^{4}$ V., Benghin ${ }^{4}$ V. \\ ${ }^{1}$ Space Research and Technology Institute, Bulgarian Academy of Sciences, Sofia, Bulgaria \\ E-mail: tdachev@bas.bg \\ ${ }^{2}$ Nuclear Physics Institute, Check Academy of Sciences, Prague, Czech Republic \\ ${ }^{3}$ Space Research Institute, Russian Academy of Sciences, Moscow, Russia \\ ${ }^{4}$ State Research Center Institute of Biomedical Problems, Russian Academy of Science, \\ Moscow, Russia
}

\begin{abstract}
The analysis of the experimental energy deposition spectra, obtained with a single detector Liulin type spectrometer (SDLTS), reveal that in the high-energy deposition channels of the spectra exist count events that cannot be explained only with the depositions from charged particles. These events are well seen in the spectra from the calibrations with protons and radionuclide neutron sources as well as in the CERN radiation field and aircraft, balloon and spacecraft spectra. If the high-energy deposition events are from protons, their energy must be mainly in the range 1-15 MeV [Simpson, 1983]. The paper reviews all mentioned above spectra and concludes that these events could be explained with the registration of neutrons in the energy range 1-100 $\mathrm{MeV}$.
\end{abstract}

\section{Introduction}

Onboard space vehicle radiation fields are composed primary of galactic cosmic rays (GCR) as well as of secondary particles, created during the nuclear interactions of the primary radiation with the matter of the spacecraft and the detector. As far as the contribution of these secondary particles to the exposure level is concerned, relatively the most important is that of neutrons. [Spurny et al., 2007].

Around the International space station (ISS), a neutron emission is produced due to the interactions of energetic charged particles of the magnetosphere with the matter of the station itself [Sevast'yanov et al., 1997]. These local neutron fluxes are present around the station. They vary along the station orbit due to the irregularity of the flux of charged particles. At balloon and aircraft altitudes, neutrons are produced also by the interaction of the primary GCR protons with the atmosphere.

\section{Instruments description}

Fig. 1 presents a generalized block diagram of a single detector Liulin type spectrometer (SDLTS) [Dachev et al., 2015]. The SDLTS usually contains one semiconductor detector, one charge-sensitive preamplifier, a fast 256-channel analogue-to-digital converter (ADC), a discriminator, a real time clock, two or more microcontrollers and a flash memory. The different modifications of Liulin spectrometers use additional modules such as visible and UV sensitive photodiodes, a temperature sensor, a Global Positioning System (GPS) module with an antenna and receiver, LED or LCD display, multimedia card (MMC) or SD card.

The measurement cycles of the SDLTS instruments is between 10 and $600 \mathrm{~s}$. During this time, one energy deposition spectrum (EDS) from the cosmic ionizing radiation is accumulated and further used for the calculation of the absorbed dose and flux in the silicon detector [Dachev, 2009]. A system international (SI) determination of the dose is used to calculate the absorbed dose in the silicon detector. The dose in SI is the energy in Joules deposited in one kilogram of a matter. The following equation relates the dose to energy loss and detector mass: 
$D=K * \sum_{1}^{256}(E i * A i) / M D$

where $M D$ is the mass of the detector (in $\mathrm{kg}$ ), $E i$ is the energy loss (in $\mathrm{J}$ ) in the channel $i, A i$ is the number of events in it, and $K$ is a coefficient. Dachev et al [2017] have already published a more comprehensive descriptions of the dose calculation procedures and the radiation sources selection procedures. Therefore, we will skip those details here.

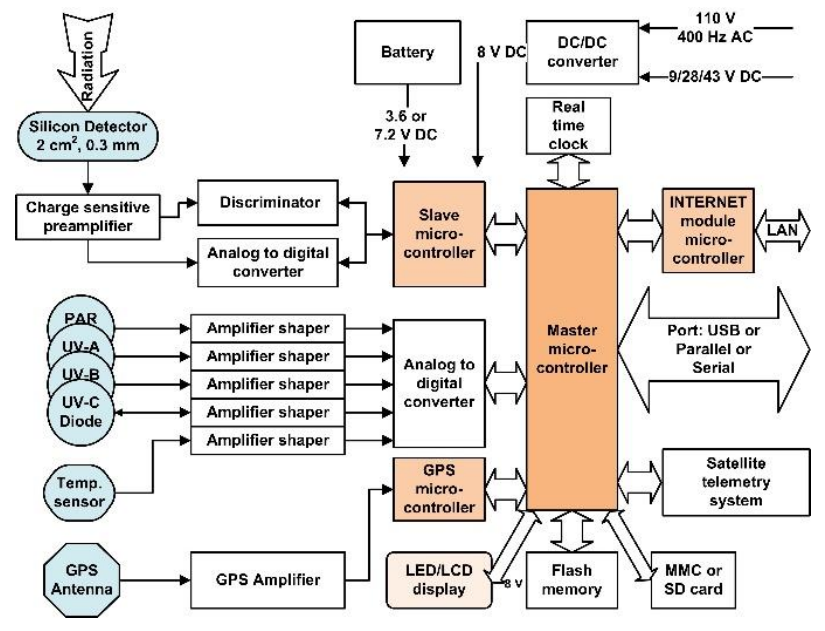

Fig. 1. Generalized block diagram of single detector Liulin type spectrometer (SDLTS).

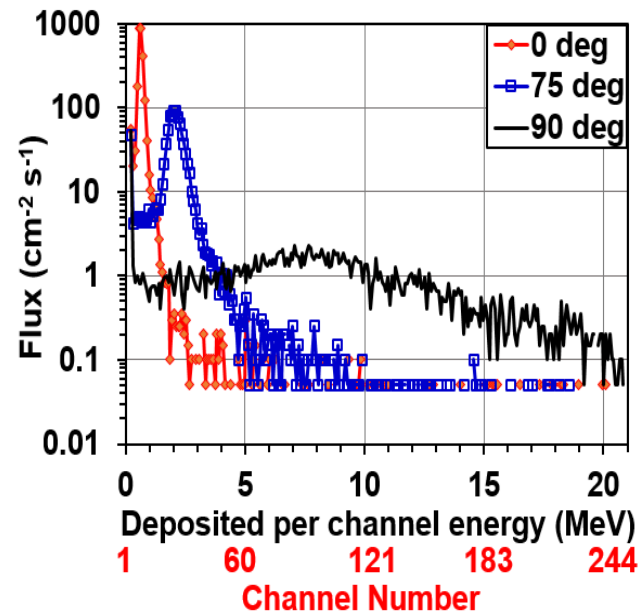

Fig. 2 Energy deposition spectra obtained during the calibrations with $32 \mathrm{MeV}$ proton flux.

\section{Methodic of neutron flux and dose recognition in the Liulin data}

\section{Recognition of the neutron events in the EDS during calibration}

Fig. 2 presents 3 energy deposition spectra obtained in the end of 1999 during the calibration of the mobile dosimetry unit number 1 (MDU-1) of Liulin-E094 instrument with a proton beam at the cyclotron facility in the Universite Catholique de Louvain, Louvain-la-Neuve, Belgium [Dachev et al., 2002]. The $65 \mathrm{MeV}$ protons pencil beam delivered by the accelerator passes through collimators, $14 \mathrm{~mm}$ graphite absorber and $1 \mathrm{~mm}$ thick scintillator.

The final proton beam was with $3-\mathrm{cm}^{2}$ area and $32 \mathrm{MeV}$ energy. The detector of the MDU was irradiated from three different incidence angles of $0^{\circ}, 75^{\circ}$ and $90^{\circ}$ against the detector's plane. The $0^{\circ} \mathrm{EDS}$ (red curve) maximum is at about $1 \mathrm{MeV}$ deposited energy, which is the smallest one because in this case the protons cross the detector with a minimal path length. The maximum of the $75^{\circ}$ spectrum is seen at $2 \mathrm{MeV}$ energy deposition. In the $0^{\circ}$ and $75^{\circ}$ spectra in the channels numbers greater than 120 single events are observed. Low energy protons or secondary neutrons could produce them, while interacting with the surrounding experimental matter including the Silicon of the detector. The same mechanism is observed in the South Atlantic Anomaly (SAA). The high-energy depositions registered in this region are produced by the long path protons and neutrons interactions with the detector. The $90^{\circ}$ spectrum is spread over the whole energy spectra of the EDS because both the primary and the secondary particles pass the longest side $(20 \mathrm{~mm})$ of the detector and produce very high-energy depositions.

To investigate more precisely the neutron sensitivity of the Si (silicium) detector in the Liulin instruments, Professor Frantisek Spurny performed series of calibrations with different neutron sources [Spurny, 2005].

Fig. 3 presents some of the results together with experimental GCR spectra obtained at aircraft and at the ISS. Seven different averaged spectra are plotted. The shortest EDS are those obtained from the ${ }^{137} \mathrm{Cs}$ gamma and the $\mathrm{AmF}$ radionuclide sources with an average energy of the neutrons of $1.5 \mathrm{MeV}$. The $2.1 \mathrm{MeV}{ }^{252} \mathrm{Cf}$ spectrum extends up to about 3.7 MeV. The average $4.2 \mathrm{MeV}$ AmBe spectrum goes up to $8 \mathrm{MeV}$ deposited energy. The longest neutron source spectrum is the CERN $50 \mathrm{MeV}$ spectrum, which covers the entire energy range. It is 
received behind a concrete shield [Mitaroff and Silari, 2002]. The region below $2 \mathrm{MeV}$ is increased because of the low energy transfer (LET) - muon particles.

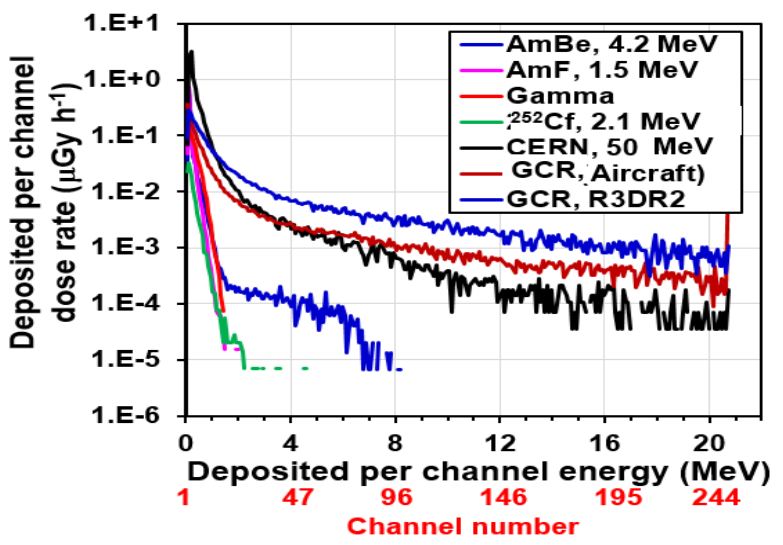

Fig. 3. Neutron sources spectra comparison.

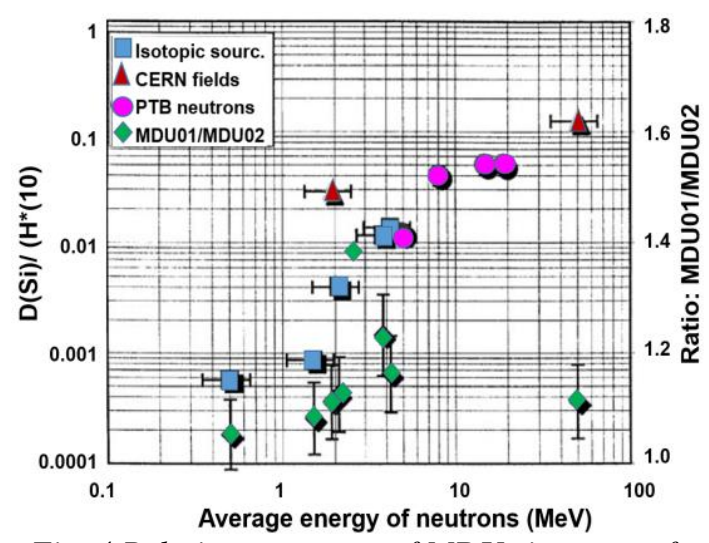

Fig. 4 Relative responses of MDUs in terms of ambient dose equivalent $(\mathrm{D}(\mathrm{Si}) / \mathrm{H} *(10)$.

The first conclusion from Fig. 3 is that the length of the neutron spectrum depends on the average energy of the neutrons in it. The second conclusion is that the spectrum above 1-1.5 $\mathrm{MeV}$ deposited energy is generated by high LET particles with a predominance of neutrons. This is confirmed from the similarities with the CERN, GCR aircraft and spacecraft spectra.

Fig 4 summarizes the results of the calibrations obtained by Prof. Spurny with two MDU units in the following three neutron fields: Isotopic neutron sources, CEC-EC high-energy reference fields [Mitaroff and Silari, 2002] and PTB Braunschweig neutrons reference beams [Guldbakke et al., 1994]. To increase the response toward neutrons, an additional polyethylene layer with a thickness of $1 \mathrm{~mm}$ was inserted before the MDU01 detector.

The total values of the doses in Si in the MDUs were calculated by means of Eq. (1). It was consider that the part of the spectrum above $1 \mathrm{MeV}$ deposited energy is populated by neutrons and neutron-like particles that is why the dose rate of this part is denoted as ambient dose equivalents $H^{*}(10)$.

The following conclusions could be drawn from Fig. 4 [Spurny, 2005]:

1. The additional polyethylene layer actually increases the response (see ratio MDU01/MDU02 in Fig. 4) maximum about 20\% for the AmBe and PuBe sources. Perhaps this is due to the direct contact of the epoxy resin layer with the Si detector. When the neutrons reach the MDU02 recoil protons are created.

2. The ratio $D(S i) / H^{*}(10)$ increases very rapidly with neutron energy. This ratio is 200 times higher for the CERN $50 \mathrm{MeV}$ field behind a concrete shield (most right red triangle point) compared with the AmLi neutron source (the most left blue quadrat point). The latter is the field with the lowest average energy of $1.5 \mathrm{MeV}$. Such behavior is expected, because the both the total neutron interaction cross section and as well as the energy transferred to high LET secondary charged particles increase substantially with neutron energy [ICRU, 2000].

The last, known yet tests of SDLTS in ${ }^{252} \mathrm{Cf}$ neutron field were performed by [Straume et al. 2016] prior to the NASA high-altitude balloon flight, the RaD-X mission [Mertens, 2016., Mertens et al. 2016]. Four radiation dosimeters were on RaD-X board: a Far West HAWK tissue equivalent proportional counter (TEPC version 3) (https://www.fwt.com/detector/fwad1ds.htm), a Teledyne dosimeter (UDOS001), a Liulin dosimeter (MDU 6SA1), and a RaySure dosimeter (version $3 b$ ). For ${ }^{252} \mathrm{Cf}$ neutron radiation, Liulin provides absorbed dose rate of $3.14 \pm 0.28 \mu \mathrm{Gy} \mathrm{h} \mathrm{h}^{-1}$ in ICRU (International Commission on Radiation Units and Measurements) soft tissue. This is lower than the observed with the TEPC dose of $5.09 \pm \mu \mathrm{Gy}$ $\mathrm{h}^{-1}$, measured on the same date. 


\section{Recognition of the neutron events in the EDS at aircraft, balloon and spacecraft}

Fig. 3 reveals that the shapes of the average CERN, aircraft and spacecraft spectra are very similar. The aircraft spectrum averages long-term data obtained between 2001 and 2005 by colleagues from Nuclear Physics Institute, Academy of Sciences of the Czech Republic [Spurny et al., 2007]. The ISS GCR spectrum is obtained between November 2014 and January 2016 by radiation risk radiometer-dosimeter (R3D)-R2 (R3DR2) instrument [Dachev et al. 2017]. The shapes of the GCR spectra curves at aircraft and spacecraft show a part with a high slope in the low energy deposition region and a smaller slope part, with an almost linear form in the energy deposition part above channel number 47 . We consider that the high slope parts of the aircraft and spacecraft spectra contain mainly high-energy charged particles, while the small slope parts are build up mainly by neutrons, sporadic low energy protons with a long path in the detector and rare hi $\mathrm{Z}$ energy (HZE) particles. The paper "A comparison of the neutron dose measurements completed by the Bulgarian and Russian instruments outside the Zvezda module of the ISS" (Dachev et al. 2020), in this issue, more comprehensively develop these ideas.

Fig. 5 and 6 visualize the single energy deposition events in the SDLTS instruments spectra at aircraft, balloon and spacecraft that are interpreted as registrations of neutron particles. These events are illustrated in Fig. 5a, 5b, 5d and Fig. 6a with single points above the dashed lines at $15^{\text {th }}$ or $48^{\text {th }}$ channels in the GCR spectra; at the $148^{\text {th }}$ channel in the inner radiation belt (IRB) and solar energetic particle (SEP) spectra and at the $24^{\text {th }}$ channel in the outer radiation belt (ORB) spectra.

Fig. 5a presents the SDLTS 600 seconds resolution parameters' measurements along the aircraft path from Prague to New York during an Intense Solar Flare, Ground Level Event 60, on April 15 $5^{\text {th }}, 2001$ [Spurny and Dachev, 2001]. Fig. 5c illustrates how the solar event produced an excess in the 'normal' exposure level due to the galactic component (as given by CARI 6) that was a little more than twice the dose rate in the silicon diode. The apparent effective dose, recalculated based on the CERN calibration, is about $80 \%$, from the event rate.
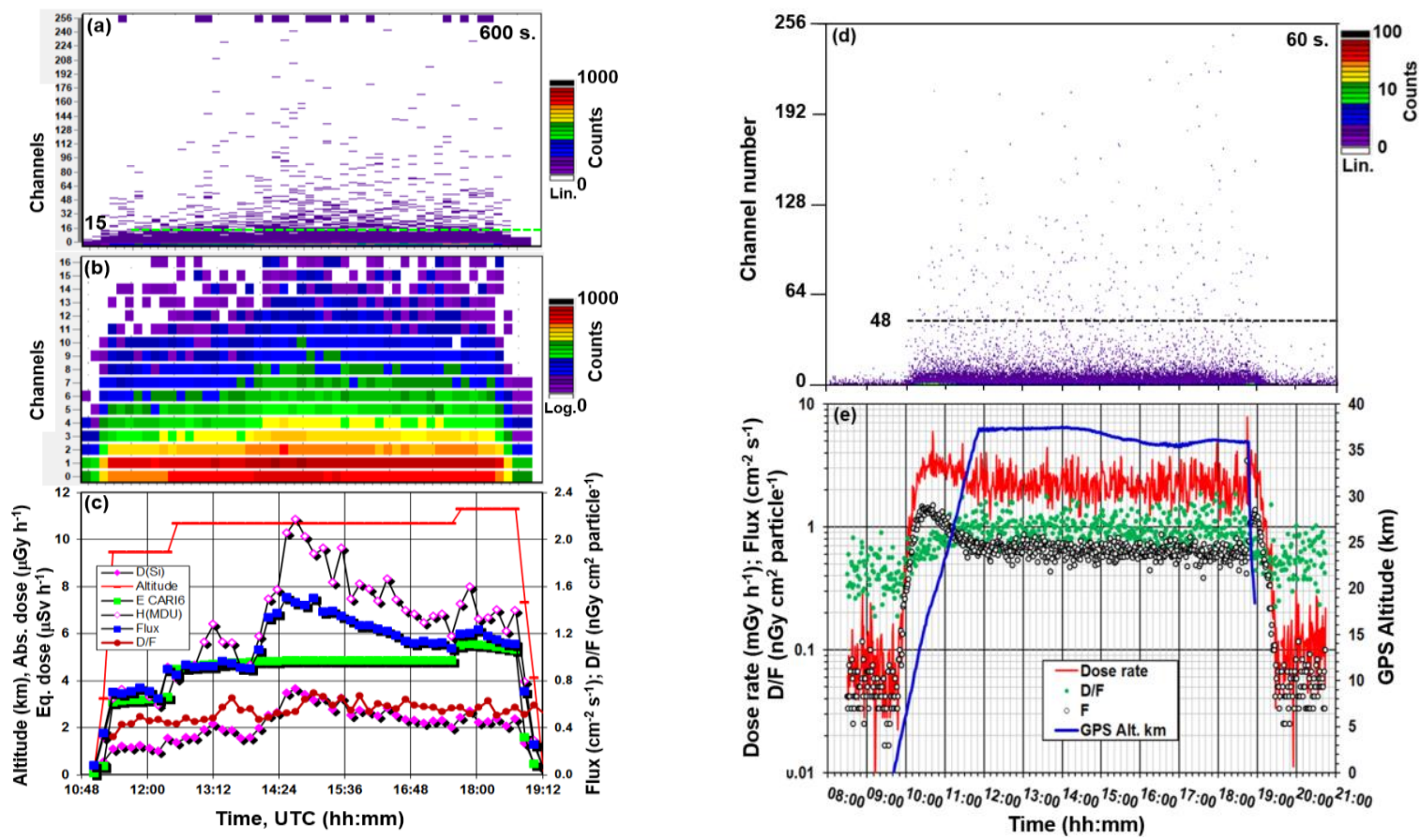

Fig. 5. Fig. 5a: 3D color-coded graphic showing the spectra channels population in the GCR radiation source in the channels range 0-256; Fig. 5b: 3D color-coded graphic showing the spectra channels population in the GCR radiation source in the channels range 0-16; Fig. 5c Analysis of the variations of the: altitude, absorbed and equivalent dose rate, flux and D/F ratio values along the aircraft trajectory on April 15, 2001; Fig. $5 d$ and $5 e$ are similar to the Fig. $5 b$ and Fig. 5c but for the balloon flight. 
The counts in all-256 channels of the spectrometer are shown on Fig. 5a. Well seen is that in the channels numbers above 15 or 48 energy deposition events (counts) are registered. Charged particles cannot explain these events. We interpret these events as neutrons. This is confirmed by the fact that these events are not observed at the altitudes below $7 \mathrm{~km}$. The same effect is even better illustrated in Fig. 5d during a balloon flight. The above assumption is also supported and is in line with Friedberg and Copeland publication (2011). The authors demonstrated that the contribution of the neutrons to the mean effective dose rate starts to dominate over the muons and other charged components at a high geographic latitude at $7 \mathrm{~km}$ altitude.

The analysis of Fig 5a and 5b reveal that enhanced fluxes and doses are registered in the neutrons channels (Fig. 5a) and in the charged particle channels (Fig. 5b) during the maximum of the solar proton flux at about 14:30 UT. The crossings of the Photzer maximum, seen in Fig. $5 \mathrm{~d}$ and $5 \mathrm{e}$, does not affect the neutron count rate.

Fig. 6 visualizes the energy deposition events in the R3DR2 instrument data that are interpret as neutron particles registrations. These events are marked in Fig. 6a with blue points above the dashed lines at $48^{\text {th }}$ channel in the GCR spectra; at $148^{\text {th }}$ channel in the IRB and SEP spectra and at $24^{\text {th }}$ channel in the ORB spectra.

Fig. $6 \mathrm{a}$ is a 3D presentation of the counts in each channel of the spectrometer from one to 256. The amount of counts is encoded with the color bar at the right-upper corner of the figure. The linear organization of the data in Fig. 6a allows the visualization of the single particles, seen as blue points in the channels of the spectrum. The dotted lines and the numbers near them denoted the upper boundary of the spectra, where the low LET particles are observed. We assume that the parts of the spectra above the dotted lines contain high-energy deposition (high LET) particles, mainly neutrons, long path low energy protons and ions heavier than $\mathrm{H}^{+}$. As well as protons penetrating trough the side walls of the detector.

Fig. 6b studies what are the absorbed and neutron dose equivalent rates and the dose to flux ratio (D/F) distribution in four typical radiation sources along the 640 spectra of the ISS orbits in May-June 2015. The data from R3DR2 instrument are used. The neutron dose equivalent rate is calculated for each source, using the methodic described in the paper by Dachev et al., "A comparison of the neutron dose measurements completed by the Bulgarian and Russian instruments outside the Zvezda module of the ISS" in this issue. The dashed black line in the bottom of Fig. $6 \mathrm{~b}$ shows the value of the dose to flux ratio D/F equal to $1-\mathrm{nGy} \mathrm{cm}^{2}$ particle ${ }^{-1}$.

The first part of measurements numbered from zero to 270 in Fig. $6 \mathrm{~b}$ shows data obtained on May 5, 2015 between 17:00:07 and 17:46:56 UT. The station crosses a region of a GCR radiation. Fig. $6 \mathrm{~b}$ illustrates that the absorbed dose rate has a maximum up to about $15 \mu \mathrm{Gy} \mathrm{h}^{-}$ ${ }^{1}$, connected with the passing of the south high latitude region with a maximal L-value of 5.33 [McIlwain, 1961]. The measured neutron dose equivalent rate (black lines and dots) follows, with larger values, the absorbed dose curve (blue). The spectra with numbers larger than 180 are with a smaller absorbed dose rate because of the crossings of the magnetic equatorial region with a minimal value of $\mathrm{L}=1.02$. In the equatorial region, the absorbed dose rate curve shows a minimum with an average value of $0.66 \mu \mathrm{Gy} \mathrm{h}^{-1}$. The equivalent dose rate curve disappears in this region because there are no counts above channel number 48 (Fig. 6a). When all of these channels are empty, the equivalent dose is zero.

The dose to flux ratio in the first 240 measurements (Fig. 6b, red line), has a values close 1$\mathrm{nGy} \mathrm{cm}^{2}$ particle $\mathrm{e}^{-1}$ because the GCR source is mainly from high-energy protons. Their energy depositions are relatively small. This is expected from the Haffner's formulae [Haffner, 1967]. A bit smaller $\mathrm{D} / \mathrm{F}$ values in the equatorial region are generated by the proton spectra with a higher average energy than in the high latitudes.

The geomagnetic shielding, measured by the vertical cutoff rigidity [Smart \& Shea, 2005], is the reason for the reducing of the GCR count rates at low L values. At the higher L values 
the vertical cutoff rigidity decreases and the major amount of the low-energy GCR particles penetrates down to the station's orbit and produces the high latitude maximum.

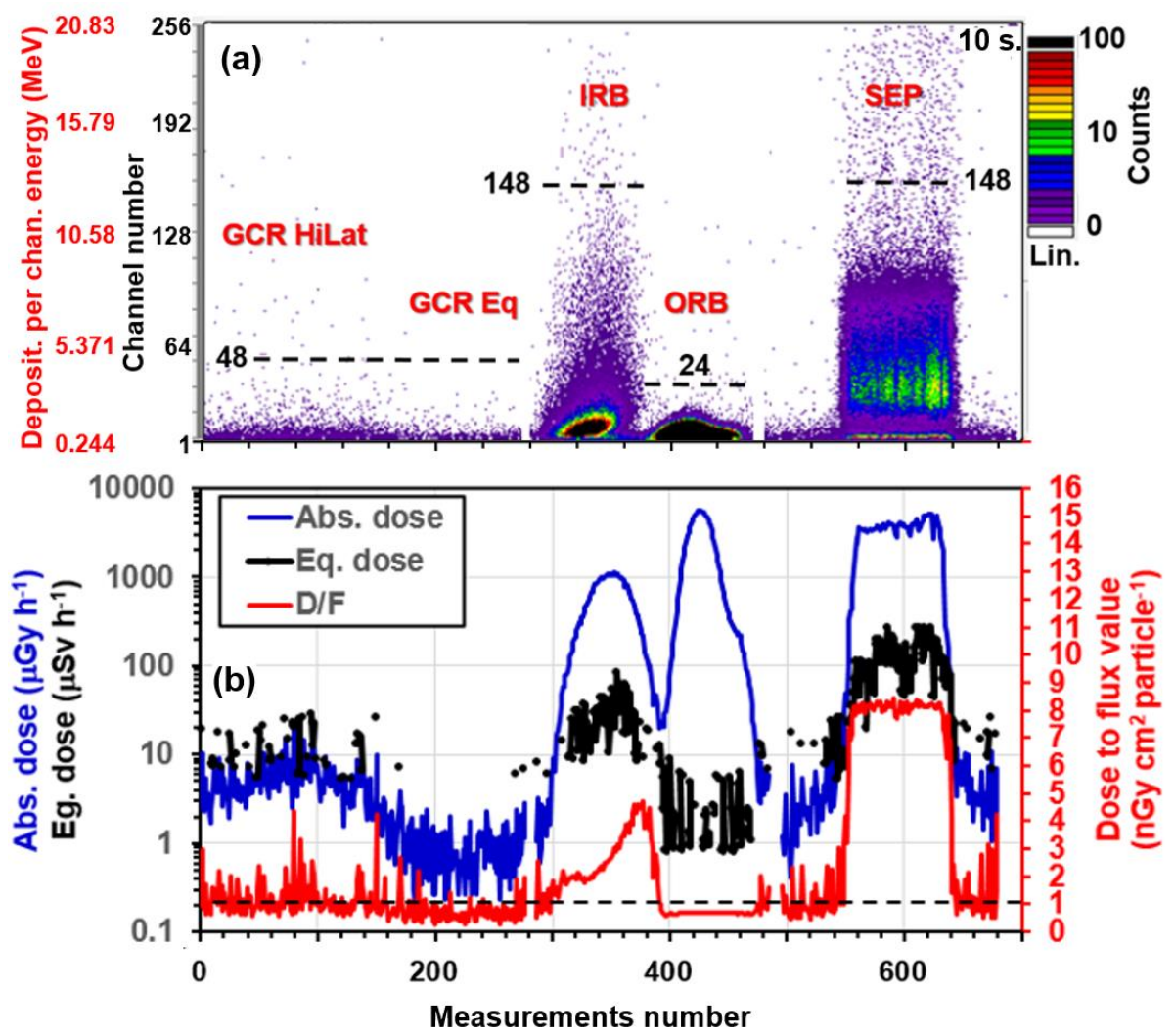

Fig. 6. Fig. 6a: 3D color-coded graphic showing the spectra channels population in different radiation sources. Fig. $6 b$ Analysis of the variations of the absorbed and equivalent dose rate values and D/F ratio along the different radiation sources seen with red labels in Fig. $6 a$.

The measurements from 280 to 480 (Fig. 6) contain data from the descending ISS orbit, which first crosses the region of the SAA and immediately after that - the outer radiation belt (ORB) region. The absorbed dose rate curve in Fig. 6b shows two maxima. The IRB maximum is smaller than the ORB. The neutron dose equivalent rate generally follows the absorbed dose rate in the region of the IRB maximum. It has about two order of magnitude smaller values. In the region of the ORB maximum the equivalent dose rate curve anti correlates the absorbed dose rate. The behavior of the $\mathrm{D} / \mathrm{F}$ ratio (red curve) confirms the findings for the dose rates. In the IRB region, the $\mathrm{D} / \mathrm{F}$ ratio is all above $1-\mathrm{nGy} \mathrm{cm}^{2}$ particle ${ }^{-1}$ because the protons, with energies 30-400 MeV, deposit higher dose than the protons from the GCR source. The latter are with much higher energies and smaller energy depositions. We calculate the values of the proton energy in the equatorial edge of the SAA maximum and receive an average value of $49 \mathrm{MeV}$, using the Haffner's formulae [Haffner, 1967]. After crossings of the SAA maximum at coordinates of $\left(44.98^{\circ} \mathrm{W}, 28.09^{\circ} \mathrm{S}\right)$ in the poleward edge of the SAA maximum, the D/F vales

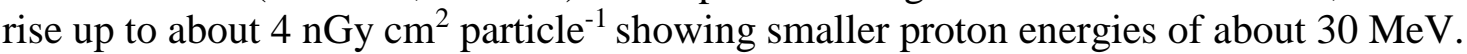

The part of the 3D graphic in Fig. 6a, which represents the SAA maximum confirms the findings above. The spectrum maximum position against the red energy deposition axis in the left part of Fig. 6a is the first with smaller energy depositions per channel, which means a higher proton energy. Further, with the movement of the ISS across the SAA the energy depositions in the maximum rise up to higher value and respectively higher channel number, meaning that the energy of the impinging protons falls down.

The large amount of blue points above the IRB maximum in Fig. 6a is generated mainly by the long path low-energy protons, populating these channels in the same way as the high-energy 
points in the inclined spectra with $75^{\circ}$ and $90^{\circ}$ in Fig. 3. We consider that mainly the secondary neutrons generate the counts above the $148^{\text {th }}$ channel.

The analysis of the spectra in the ORB maximum in Fig 6a shows that they are with a single maximum at the low energy depositions as expected from the relativistic electrons. Above the channel 24 there are very small number of counts, probably generated by sporadically GCR protons. All D/F values in the ORB region (Fig. 6b) are smaller than 1-nGy $\mathrm{cm}^{2}$ particle $^{-1}$, as expected from the Haffner's formulae [Haffner, 1967].

The SEP maximum (last part of data in Fig. 6) is obtained between 20:02:34 and 20:16:14 UT on 22 June 2015. It is the highest maximum of this SEP event with a maximal dose rate of 5,242- $\mu \mathrm{Gy} \mathrm{h} \mathrm{h}^{-1}$ at 20:13:44 UT. The spectra in Fig. 6a shows small maximum in the first channels generated by the GCR particles. Further, there is a broad maximum in channels 30 90, generated by the $7 \mathrm{MeV}$ SEP protons at the detector surface (Dachev et al., 2016).

\section{Conclusions}

The purpose of this paper is to demonstrate that a single detector Liulin type spectrometer is able to register neutrons in the energy range 1-100 MeV.

The presented evidences from the calibrations with protons and radionuclide neutron sources as well as from the CERN radiation field and aircraft, balloon and spacecraft spectra confirm that the Liulin type instruments do have the sensitivity to detect neutrons with energies above $1 \mathrm{MeV}$. The SDLTS is used for a neutron dose equivalent rate calculation, measured with the R3DR instrument on the ISS, and for a comparison with the Russian BTN-NEUTRON instrument data. The results are presented in the paper by Dachev et al., named "A comparison of the neutron dose measurements completed by the Bulgarian and Russian instruments outside the Zvezda module of the ISS" in this issue.

\section{Acknowledgements}

This work is supported by: Agreement between RAS and BAS on "Fundamental space research", Contract No. 4000117692/16/NL/NDe Funded by the Government of Bulgaria through an ESA Contract under the PECS (Plan for European Cooperating States), Contract with National Science Fund -Bulgaria on Project N 129 FOR BILATERAL PROJECTS BULGARIA - RUSSIA 2018 -2019. In IKI-RAS the work is supported by the Russian Science Foundation under grant 19-52-18009._NPI work was funded by the European Structural and Investment Funds under the Operational Program Research, Development and Education (project CRREAT CZ.02.1.01/0.0/0.0/15_003/0000481).

Most of the data used in this paper are part of the "Unified web-based database with Liulintype instruments", which are available online, free of charge at the following URL: http://esapro.space.bas.bg/database. The database was created under the ESA contract mentioned above.

\section{References}

Dachev Ts., B. Tomov, Yu. Matviichuk, Pl. Dimitrov, J. Lemaire, Gh. Gregoire, M. Cyamukungu, H. Schmitz, K. Fujitaka, Y. Uchihori, H. Kitamura, G. Reitz, R. Beaujean, V. Petrov, V. Shurshakov, V. Benghin, F. Spurny, (2002), Calibration Results Obtained With Liulin-4 Type Dosimeters. Adv. Space Res. 30, 4, 917-925. http://dx.doi.org/10.1016/S0273-1177(02)00411-8.

Dachev, Ts.P., (2009), Characterization of near Earth radiation environment by Liulin type instruments, Adv. Space Res., 44, 1441-1449. http://dx.doi.org/10.1016/j.asr.2009.08.007

Dachev, T.P., J.V. Semkova, B.T. Tomov, Yu.N. Matviichuk, Pl.G. S. Maltchev, R. Koleva, Pl., Dimitrov, N.G. Bankov, V.V., Shurshakov, V.V., Benghin, E.N., Yarmanova, O.A. Ivanova, D.-P. Häder, M.T. Schuster, G. Reitz, G. Horneck, Y. Uchihori, H. Kitamura, O. Ploc, J. Kubancak, I. Nikolaev, (2015), Overview of the Liulin type instruments for space radiation measurement and their scientific results, 92-114. http://dx.doi.org/10.1016/j.1ssr.2015.01.005.

Dachev, T. P., Bankov, N. G., Tomov, B. T., Matviichuk, Y. N., Dimitrov, P. G., Häder, D.-P. \& Horneck, G., (2017), Overview of the ISS radiation environment observed during the ESA EXPOSE-R2 mission in 20142016. Space Weather, 15, 1475-1489. https://doi.org/10.1002/2016SW001580. 
Friedberg, W. and Copeland, K., (2011). Ionizing Radiation in Earth's Atmosphere and in Space Near Earth, Oklahoma City, OK: Federal Aviation Administration, Civil Aerospace Medical Institute DOT Report No. DOT/FAA/AM-11/9.

Guldbakke, S., Dietz, E., Kluge, H., Schlegel, D., (1994). PTB Neutron Fields for the Calibration of Neutron Sensitive Devices, Vol. I. Physik und Messtechnik, Strahlenschutz, pp. 240-247 (Koln, Verlag TUV Rheinland).

Haffner, J. W., (1971). Yadernoe izluchenie i zashchita v kosmose (Nuclear Radiation and Protection in Space), pp 115, Atomizdat, Moscow. (book in Russian)

ICRU, (2000). Nuclear Data for Neutron and Proton Radiotherapy and for Radiation Protection, Report 63. ICRU Publication, Bethesda, MD.

McIlwain, C.E., (1961). Coordinates for mapping the distribution of magnetically trapped particles. Journal of Geophysical Research, 66,3681-3691.

Mertens, C.J., (2016). Overview of the radiation dosimetry experiment (RaD-X) flight mission. Space Weather, 14(11), 921-934.

Mertens, C.J., et al. (2016). Cosmic radiation dose measurements from the RaD-X flight campaign, Space Weather, 14, 874-898, https://doi.org/10.1002/2016SW001407.

Mitaroff, A., Silari, M., (2002). The CERN-EU high-energy reference field (CERF) facility for dosimetry at commercial flight altitudes and in space. Radiat. Prot. Dosim. 102, 7-22.

Sevast'yanov, V.D., Tarnovskii, G.B., and Lyagushin, V.I., (1997). Measurement of the Neutron Energy Spectrum on the Mir Orbital Station, Kosm. Issled., 35, 2, 216-220. [Cosmic Research, pp. 201-205].

Simpson, J. A. (1983). Composition and origin of cosmic rays. In M. M. Shapiro (Ed.), NATO ASI Series, Series C Mathematical and Physical Sciences (Vol. 107, pp. 1-24). Dordrecht: Reidel.

Smart, D.F. and Shea, M.A., (2005). A review of geomagnetic cutoff rigidities for earth-orbiting spacecraft. Advances in Space Research, 36(10), 2012-2020.

Spurný, F., (2005). Response of a Si-diode-based device to fast neutrons, Radiation Measurements, 39, 219 - 223. Spurný, F., Ploc O., Dachev, T., (2007). On the Neutron Contribution to the Exposure Level onboard Space Vehicles, Radiation Prot. Dosimetry, 126, 519 - 523. http://rpd.oxfordjournals.org/content/126/1-4/519

Straume, T., C. J. Mertens, T. C. Lusby, B. Gersey, W. K. Tobiska, R. B. Norman, G. P. Gronoff, and A. Hands (2016), Ground-based evaluation of dosimeters for NASA high-altitude balloon flight, Space Weather, 14, https://doi.org/10.1002/2016SW001406. 


\title{
Giant Ukrainian Radio Telescope as an Instrument for Interplanetary Scintillation Observations
}

\author{
Kalinichenko ${ }^{1,2,}$ M.M., Kuhai ${ }^{2,1}$ N.V., Konovalenko ${ }^{1}$ O.O., \\ Bubnov ${ }^{l}$ I.M., Erin ${ }^{l}$ S.M., Romanchuk ${ }^{l}$ O.I. \\ ${ }^{1}$ Institute of Radio Astronomy of NASU, Kharkiv, Ukraine; \\ ${ }^{2}$ Hlukhiv national pedagogical university, Hlukhiv, Ukraine \\ E-mail: kalinich@rian.kharkov.ua
}

The potential of the radio telescope GURT in its present state for interplanetary scintillation (IPS) observations is discussed. We estimated the minimum detectable flux density in the case when two subsections of the radio telescope GURT are used. Corresponding test experiments have been also carried out. This work is interesting from point of view of expanding the global IPS network.

\section{Introduction}

The beginning of the new millennium is marked by a sharp increase in interest in low-frequency radio astronomy. This is due, among other things, to the importance of the effects and cosmic objects that radio astronomy studies. So it is at low frequencies there are strong interaction of emission and space plasma; significant excess of non-thermal over thermal radio emission intensity, formation of many types of non-thermal radio emission with steep spectra. Some types of sporadic solar and planetary radio emission also have maximum at decameter - meter wavelengths. The increased interest is accompanied by the construction of a number of new unique radio telescopes such as Low Frequency Array (LOFAR), the Netherlands, 30 to 80 and 110 to $240 \mathrm{MHz}$ [van Haarlem et al., 2013], the Long Wavelength Array (LWA), USA, 10 to $88 \mathrm{MHz}$ [Taylor et al., 2012], the Murchison Wide-field Array (MWA), Australia, 80 to 300 $\mathrm{MHz}$, new extension in Nançay Upgrading LOFAR (Nenu-FAR), France, 10 to 85MHz [Zarka et al., 2012]. A similar radio telescope GURT is being created in Ukraine [Konovalenko et al., $2016 \mathrm{a}, \mathrm{b}$ ]. These instruments are already being used for investigations of the Sun, the Earth's ionosphere, and solar-terrestrial connections.

The aim of this paper is to estimate the perspectives of using the radio telescope GURT for interplanetary scintillation studies.

\section{The radio telescope GURT}

GURT is being built at S. Y. Braude radio astronomical observatory in Kharkiv region, Ukraine (Fig.1). It is a phased array composed of identical subarrays consisting of 25 active antenna elements. Each antenna element is a crossed dipole (Fig.2a). The distance between antenna elements is $3.75 \mathrm{~m}$, their height above the ground is $1.6 \mathrm{~m}$. The subarray design provides a wide operational frequency range from 8 to $80 \mathrm{MHz}$, high sensitivity (the galactic background level exceeds their self-noise by more than $7 \mathrm{~dB}$ ), and high interference immunity (achieved due to the high dynamic range of the dipole amplifier: input IP3 of $30 \mathrm{dBm}$ ). Subarrays are currently being added, with the finished array planned to incorporate 100 subarrays for a total of 2500 elements. As number of subarrays and effective area increase the number of astrophysical problems to be solved with GURT will increase significantly too. For receiving and postprocessing signals each GURT subarray is equipped with a wideband digital receiver (Advanced Digital Receiver, Fig. 2b) [Vasilyev et al., 2014]. The receiver parameters are well matched with the parameters of the GURT subarray and allow us to receive the cosmic signals in whole frequency band (from 8 to $80 \mathrm{MHz}$ ) with high time-frequency resolution and high dynamic range. Receivers have the high sensitivity and low level of intrinsic noise. The radio telescope GURT is planned to be equipped with additional facilities aimed at participation in 
joint international observation campaigns using other large radio telescopes located worldwide. As at the end of 2020, there is no phasing system for subarrays yet, it is possible to use either one subarray or two subarrays with multiplying signals of one polarization.

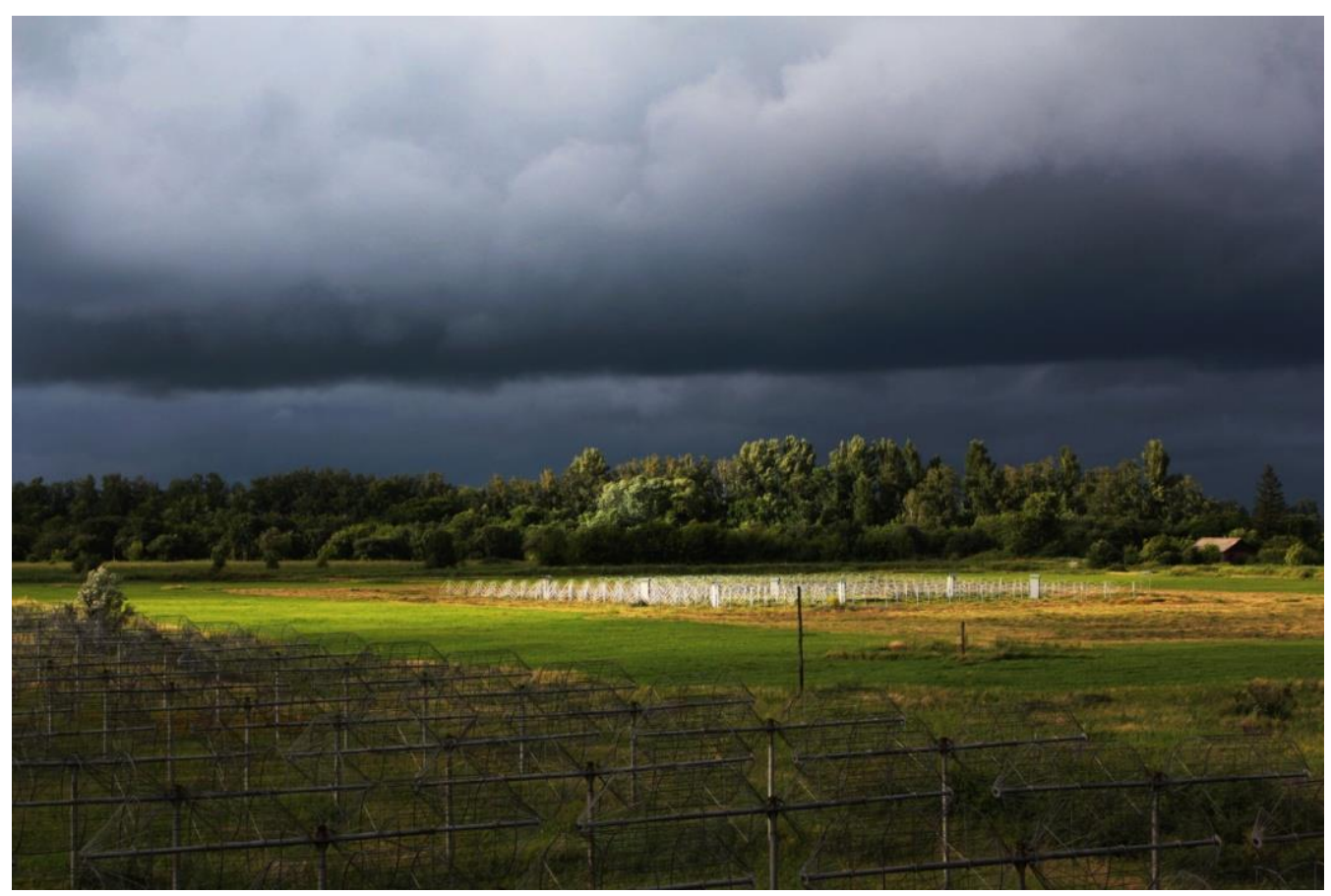

Fig.1. North arm of the radio telescope UTR-2 (foreground) and the radio telescope GURT behind it (white color). S. Y. Braude radio astronomical observatory

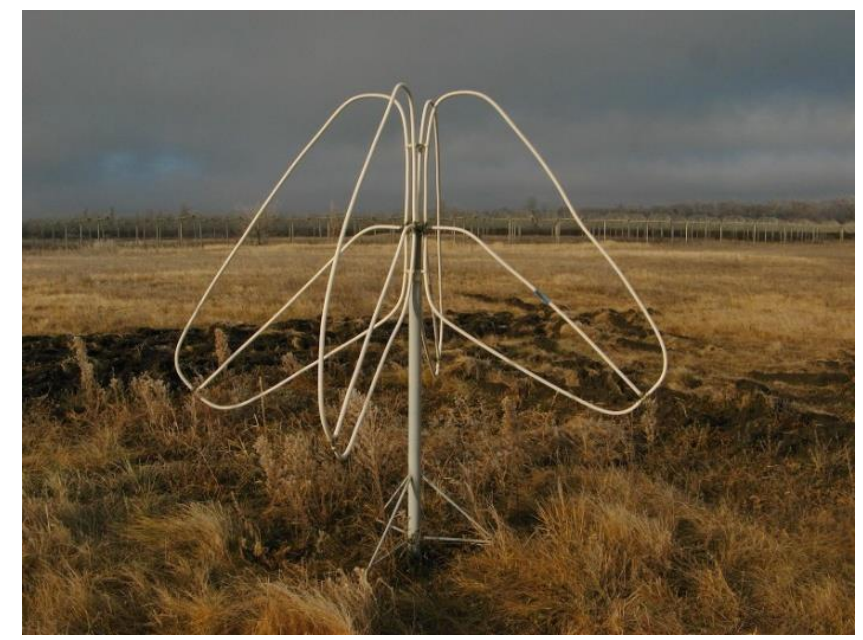

a)

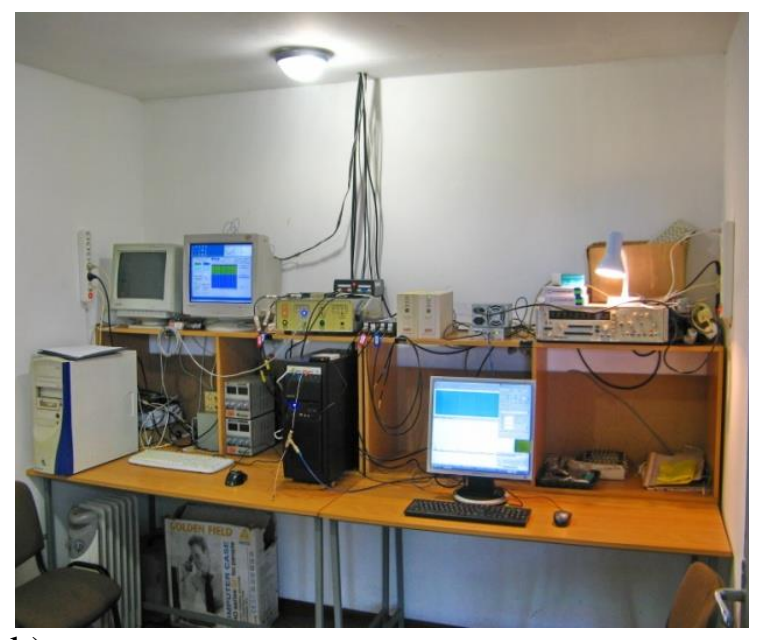

b)

Fig.2. a) An antenna element of the radio telescope GURT, b) laboratory room with receiving equipment

Possibilities of electronic scanning in wide sectors, high sensitivity in a wide frequency range and high interference immunity have allowed experiments to be carried out by using only one or two subarrays (observations of sporadic solar and planetary radio emission or observations of radio pulsars) [Konovalenko et al., $2016 \mathrm{a}, \mathrm{b}$ ]. The aim of this paper is to estimate the perspectives of using the radio telescope GURT for the study of the interplanetary scintillations.

\section{Interplanetary scintillations}


InterPlanetary Scintillations (IPS) is the random intensity fluctuations of the radio emission of cosmic radio sources (quasars, radio galaxies, pulsars) caused by diffraction of the wave front in the interplanetary plasma. A. Hewish was the first who observed the interplanetary scintillations [Hewish et al., 1964]. Observations of the interplanetary scintillations allow us to measure the interplanetary turbulence parameters as well as to estimate the angular structure of radio sources [Kojima et al., 1982, Manoharan et al., 1990, Breen et al., 1996, Fallows et al., 2008]. IPS observation also is a powerful tool for studying interplanetary coronal mass ejections (ICME) and other solar wind disturbances connected with solar activity. At decameter wavelengths IPS observations are regularly carried out in Institute of Radio Astronomy of NAS of Ukraine by using UTR-2 - URAN radio telescopes [Konovalenko et al., 2016 a] for the last 20 years [Kalinichenko, 2009; Falkovich et al., 2010; Kalinichenko et al., 2017; Kalinichenko et al., 2019].

Now for IPS observations it is possible to use two subarrays of the radio telescope GURT with multiplying signals of one polarization. Let us estimate the minimum flux that a source should have in this case. It can be done in the following way.

The power spectra of the IPS signal and the noise are expressed by a power law:

$$
P_{s, n}(f)=\frac{1}{1+\left(f / F_{s, n}\right)^{\alpha_{s, n}}},
$$

where the indices "s" and " $\mathrm{n}$ " refer to the signal and the noise, respectively. $\alpha_{n}$ is equal to 2 and $F_{n}=1 / 2 \pi \tau$ ( $\tau$ is the time constant). $\alpha_{s}$ varies from 2 to $4, F_{s} \approx 0.3 \mathrm{~Hz}$. The expression for the dynamic range of $P_{s}(f)$ can be written as:

$$
\frac{P_{s}(0)}{P_{n}(0)}=\gamma \frac{\sigma_{s}^{2}}{\sigma_{n}^{2}} \frac{F_{n}}{F_{s}}=\frac{\gamma}{2 \pi \tau F_{s}} \frac{\sigma_{s}^{2}}{\sigma_{n}^{2}},
$$

where $\sigma_{s}^{2}=\frac{m^{2} S^{2} A_{e f}^{2}}{4 k^{2}}, \sigma_{n}^{2}=\frac{T_{s y s}^{2}}{\Delta f \tau}, S$ is the flux density, $A_{e f}$ is the effective area, $T_{s y s}$ is the system noise temperature (it is equal to the galactic background temperature), $\Delta f$ is the observing bandwidth, $k$ is Boltzmann's constant $\left(k=1.38 \cdot 10^{-23}\right.$ Дж/K). The coefficient $\gamma$ ranges from 1 to 1.4 and takes into account the variations of $\alpha_{s}$. The variance of the periodogram estimate at any frequency is known to be equal to the square of its expectation value at that frequency (or the standard deviation is 100 percent of the value). The variance of the averaged estimate is smaller than the estimate itself by a factor of $1 / N$, where $\mathrm{N}$ is the number of the averaged spectra. As we usually use $\mathrm{N}=10, P_{s}(0) / P_{n}(0)$ has to be larger than 100 for interplanetary plasma parameters can be effectively estimated. The minimum detectable flux density $S_{\text {min }}$ (in Jy) for $P_{s}(0) / P_{n}(0)=100$ and $\gamma=1.2$ (mean value) can thus be estimated as:

$$
S_{\min }=46 \frac{k T_{\text {sys }}}{m A_{\text {eff }}} \sqrt{\frac{F_{s}}{\Delta f}} .
$$

$S_{\min }$ is equal to about $800 \mathrm{Jy}$ for the parameters: $A_{\text {eff }}=225 \cdot \mathrm{m}^{2}, \mathrm{~m}=0.2, T_{\text {sys }}=8 \cdot 10^{3} \mathrm{~K}$ (for the frequency of $45 \mathrm{MHz}$ in the middle part of the GURT frequency band (10 to $80 \mathrm{MHz}$ ), $\Delta f=70 \mathrm{MHz}$.

\section{Observations}


Only one compact radio source has such a powerful flow. This is a compact radio source in Crab Nebula. Crab Nebula is the most famous and well studied supernova remnant formed from the star that exploded in the year 1054 AD. It is identified as a strong source of x-ray, optical and radio emission. At radio frequencies this supernova remnant is associated with the radio source 3C144. The compact radio source in Crab Nebula is known to be associated with the well-known pulsar PSR B0531 +21. The pulsar flux at decameter wavelengths is about 1000 Jy and the angular size of approximately 2 arcsec.

The observations of Crab Nebula was carried out at hour angles $1.5 \mathrm{hr}$ near the upper culmination with scans lasting 20 min. Records were obtained by using Advanced Digital Receiver which allows us to carry out spectral analysis of a band up to $80 \mathrm{MHz}$ wide (at a sampling frequency of $160 \mathrm{MHz}$ ) with high frequency and time resolutions. Fig. 3 shows examples of dynamic scintillation spectra (intensity in the time/frequency plane) obtained in the process of the observations. It can be seen that the Crab Nebula showed both ionospheric (long-period) and interplanetary (short-period) scintillations.

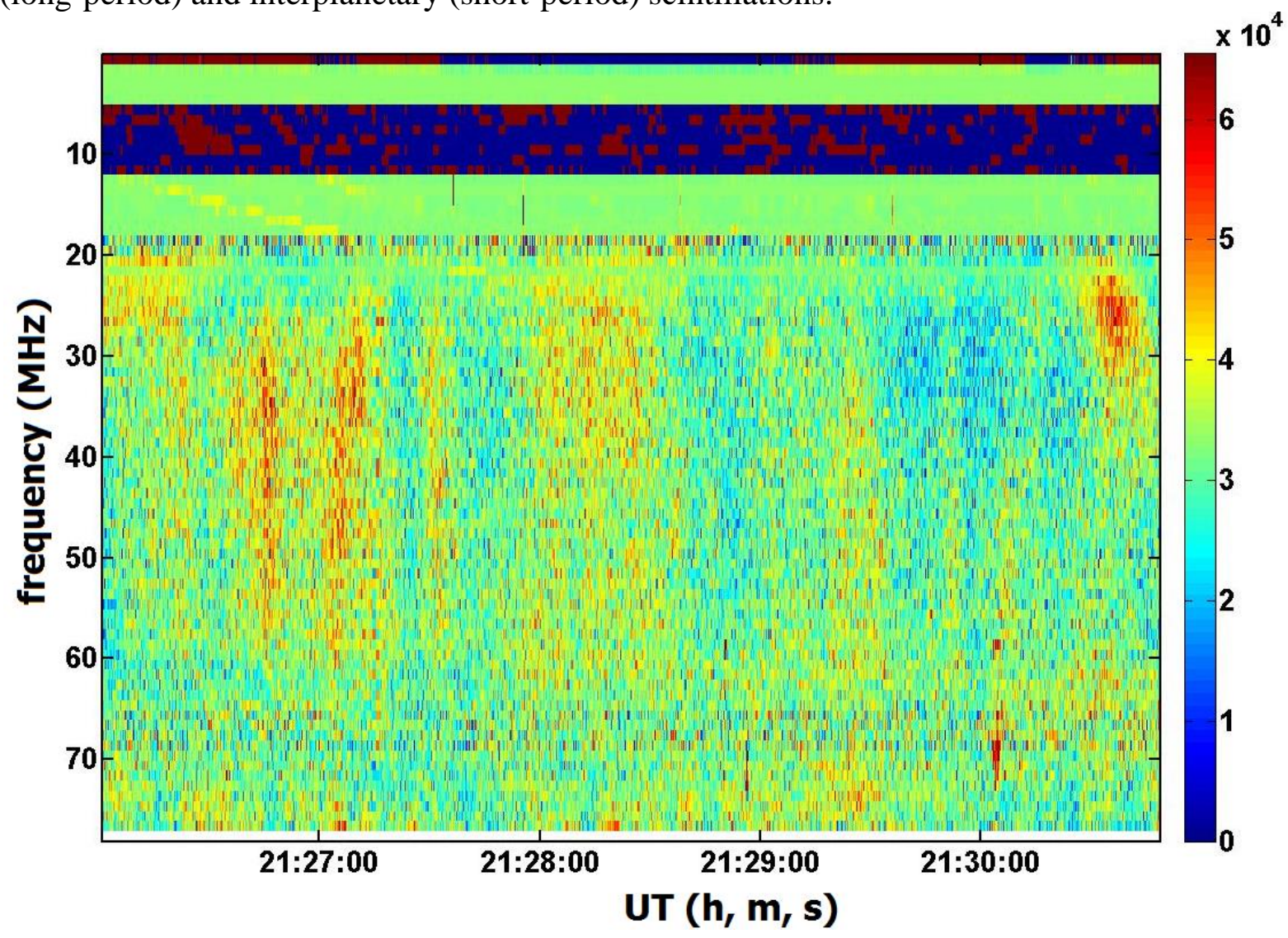

Fig.3. Dynamic scintillation spectrum of Crab Nebula

Let us estimate the scintillation spectrum by:

$$
P(v)=|F(v)|^{2} / T,
$$

where $F(v)$ is Fourier transform of scintillation process, $v$ is the fluctuation frequency, $T$ is the duration of time series $(20 \mathrm{~min})$.

Fig. 4 shows an example of daily averaged scintillation spectrum of Crab Nebula. 


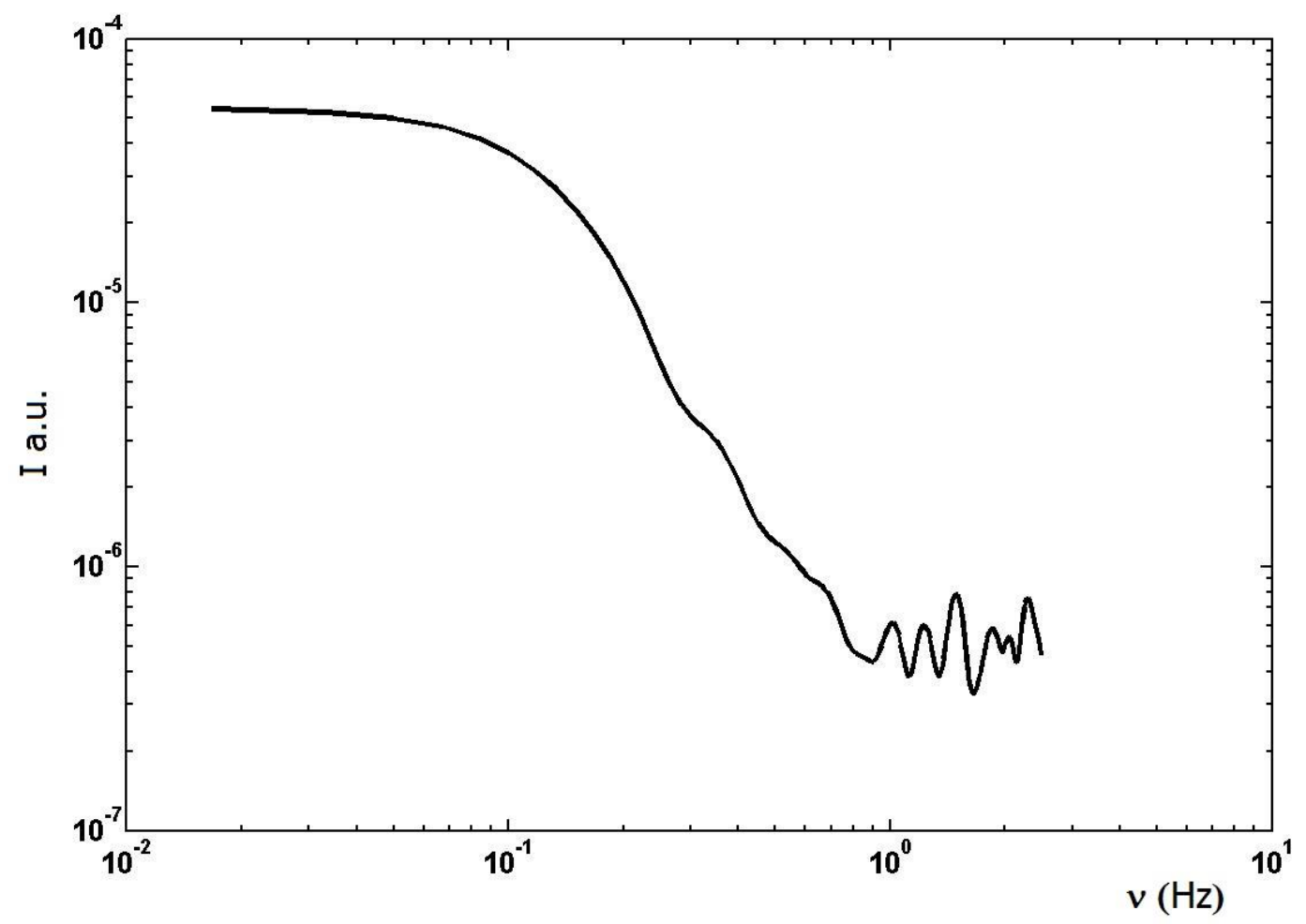

Fig.4. Scintillation spectrum of Crab Nebula

It can be seen that observed scintillation spectrum of Crab Nebula consists of low and highfrequency parts associated with Earth's ionosphere and interplanetary plasma correspondingly.

\section{Conclusions}

The carried out investigations show the possibility to register interplanetary scintillations with only two subarrays of the radio telescope GURT. As the number of GURT subarrays increases, the sensitivity of IPS measurements will increase too. So joint observations of the same radio sources with GURT, UTR-2 - URAN and LOFAR radio telescopes will become possible. Such long - base observations can provide new information on the interplanetary plasma and physical processes in it.

\section{References}

Breen, A.R., Coles, W.A., Gral, R.R.1, Klinglesmith, M.T., Markkanen, J., Moran, P.J., Tegid, P., Williams, P.J.S. (1996). EISCAT measurements of the solar wind", Annales Geophysicae, 14, pp. 1235-1245, 1996.

Falkovich, I.S., Konovalenko, A.A., Kalinichenko, N.N. et al. (2010). Dispersion Analysis of Interplanetary Scintillations at Decameter Wavelengths: First Results, Radio Physics and Radio Astronomy, 1, issue 1, pp. 39, 2010 (in Russian).

Fallows, R.A., Breen, A.R., Dorrian, G.D. (2008). Developments of the use of EISCAT for interplanetary scintillations, Annales Geophysicae, 26, issue 8, pp. 2229-2236, 2008.

van Haarlem, M.P., et al. (2013). LOFAR: The LOw-Frequency ARay. Astronomy and Astrophysics. Astronomical instrumentation, 556, A2, 53p., 2013.

Hewish, P.F. Scott, and D. Wills. (1964). Interplanetary Scintillation of Small Diameter Radio Sources, Nature, 203, 4951, pp. 1214-1217, 1964.

Kojima, J., Ishida, Y., Maruyama, K., Kakinuma, T. (1982). An observation system of interplanetary scintillation at UHF, Proc.Res. Inst. Atmospherics of Nagoya Univ. 29, p. 61, 1982.

Kalinichenko, N.N. (2009). A search for compact decametric radio sources in supernova remnants using the interplanetary scintillation technique, Astrophysics and Space sciences, 319, issue 2-4, pp. 131-138, 2009.

Kalinichenko, N.N., Olyak, M.R., Konovalenko, A.A. et al. (2017). The investigations of the solar wind beyond Earth's orbit by IPS observations at decameter wavelengths: Present state and perspectives ". Planetary Radio Emissions VIII, Proceedings of the 8th International Workshop held at Seggauberg, Austria, October 25-27, 
2016. Edited by G. Fischer, G. Mann, M. Panchenko, and P. Zarka. Austrian Academy of Sciences Press, Vienna, p. 479-486, 2017.

Kalinichenko, N.N., Olyak, M.R.,.Konovalenko, A.A., Brazhenko, A.I., Kuhai, N.V., Romanchuk, A.I. (2019). Large-Scale structure of solar wind beyond the Earth's orbit: reconstruction using the data of two-site measurements of interplanetary scintillations in the decameter radio Range", Kinematics and Physics of Celestial Bodies, 35, issue 1, pp. 17-27, 2019.

Konovalenko, A.A., et al. (2016 a). The modern radio astronomy network in Ukraine: UTR-2, URAN and GURT, Experimental Astronomy, 42, 1, pp. 11-48, 2016.

Konovalenko, A.A., Yerin, S.N., Bubnov, I.N., Tokarsky, P.L., Zakharenko, V.V., Ulyanov, O.M., Sidorchuk, M.A., Stepkin, S.V., Gridin, A.A., Kvasov, G.V., Koliadin, V.L., Melnik, V.N., Dorovskyy, V.V., Kalinichenko, N.N. 1, Litvinenko, G.V., P. Zarka, P., Denis, L., Girard, J., Rucker, H.O., Panchenko, M., Stanislavsky, A.A., Khristenko, O.D., Mukha, D.V.,Reznichenko A.M., Lisachenko, V.M., Bortsov, V.V., Brazhenko, A.I., Vasylieva, I.Y., Skoryk, A.O., Shevtsova, A.I., and Mylostna, K.Y. (2016 b). Astrophysics studies with small low-frequency radio telescopes of new generation. Radio Physics and Radio Astronomy, 21, 2, pp. 83-131, 2016 (in Russian).

Manoharan, P.K., Ananthakrishnan, S. (1990). Determination of solar-wind velocities using single - station measurements of interplanetary scintillations, MNRAS, 244, pp. 691 - 695, 1990.

Taylor, G. B., Ellingson, S. W., Kassim, N. E. et al. (2012). First light for the first station of the long wavelength array, Journal of Astronomical Instrumentation, 01, 01, 2012.

Vasilyev, O.Y., Kuzin, A.I., Kravtsov, A.A., Bulakh, E.V., Vinogradov, V.V. and Vavriv, D.M. (2014). Multifunctional Digital Receiver-Spectrometer. Radio Physics and Radio Astronomy, 19, 3, pp. 276-289, 2014 (in Russian).

Zarka, P., Girard, J. N., Tagger, M., Denis, L. \& the LSS team (2012) LSS/NenuFAR: The LOFAR super station project in Nançay, in Annual Meeting of the French Society of Astronomy and Astrophysics, Boissier, S., de Laverny, P., Nardetto, N., Samadi, R., Valls-Gabaud, D. \& Wozniak, H. (eds.) (Nice, France), p. 687. 2012. 


\title{
Decadal NAO Oscillations Excited by TSI Cycles
}

\author{
Chapanov Ya.
}

Climate, Atmosphere and Water Research Institute, Bulgarian Academy of Sciences, Sofia, Bulgaria

E-mail: yavor.chapanov@gmail.com

\begin{abstract}
.
The solar activity strongly affects climate cycles by the Total Solar Irradiance (TSI) variations. The common solar and climate cycles are studied by means of multi-decadal winter North Atlantic Oscillation (NAO) reconstruction for the period 1049-1995. The winter NAO variations are based on a speleothem-based precipitation proxy from Scotland and a tree-ring based drought proxy from Morocco and reflect precipitation regimes over the Millennium. A part of this reconstruction is compared with the TSI variations after 1610 in different decadal frequency bands. The time series are analyzed by the Method of Partial Fourier Approximation (PFA), and common cycles of TSI and $\mathrm{NAO}$ are determined in 12 frequency bands with periods between 8.5 and 50yr.
\end{abstract}

\section{Introduction}

The solar activity affects various processes on Earth surface, oceans and atmosphere. The solar cycles are connected with significant TSI variations, whose direct effect on the Earth is visible as climate and weather change. The shape of 11-year TSI cycles is not sinusoidal, so the solar-terrestrial influences are driven by various harmonics with interannual, decadal and centennial periodicity.

The North Atlantic Oscillation is a regional process, whose weather and climatic variations have long been detected and studied. The NAO is a complex climatic phenomenon with a wide range of periodicities in meteorological parameters as temperature and pressure fields, atmospheric precipitation, tropical cyclone activity, wind direction, and wind strength. The NAO is the dominant mode for winter climate variability over a vast region that extends from central North America to Europe and also involves a considerable part of northern Asia. The NAO is part of the Arctic Oscillations and is also geographically related to the Atlantic Multidecadal Oscillation (AMO), which is caused by variations in the North Atlantic surface temperature.

In order to predict climatic changes in this region, it is necessary to study the spatial and temporal dynamics of NAO, and to determine the causes of its external excitation. Significant influence of TSI on long-term NAO variations with periodicities above 50 years is determined in (Gorshkov and Chapanov, 2020). Here the TSI influence on NAO cycles with periods below 50 years is studied.

\section{Data}

The TSI and NAO data are presented in Fig.1. The estimated solar irradiances for the last 400 years are based on the NRLTSI2 historical TSI reconstruction model by J. Lean (Fig. 1, bold line), the last version in (Coddington at al. 2016). The Winter NAO reconstruction (Fig. 1 , dashed line) is based on decadal scale variations in a speleothem precipitation proxy from Scotland and a tree-ring drought proxy from Morocco. It reflects quasi-regular occurrences of contrasting precipitation regimes over the Millennium. These proxy records are located centrally in the opposing poles of NAO driven precipitation regimes (Trouet et al., 2009). 


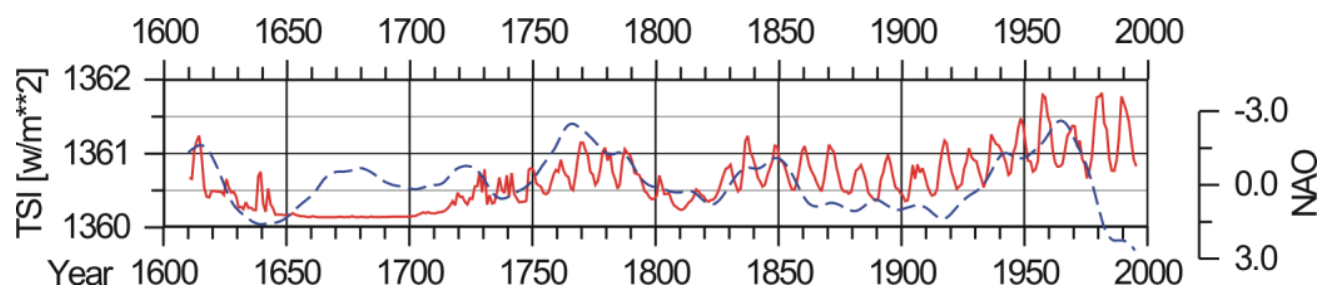

Fig.1. Time series of TSI (solid red line) and NAO (dashed blue line).

\section{Methods}

The time series spectra are determined by the well-known Fast Fourier Transform (FFT). The periodical variations are derived from the data by means of partial Fourier approximation based on the Least-Squares estimation of Fourier coefficients. The Partial Fourier approximation $F(t)$ of time series is given by

$$
F(t)=f_{0}+f_{1}\left(t-t_{0}\right)+\sum_{k=1}^{n} a_{k} \sin k \frac{2 \pi}{P_{0}}\left(t-t_{0}\right)+b_{k} \cos k \frac{2 \pi}{P_{0}}\left(t-t_{0}\right),
$$

where $P_{0}$ is the period of the first harmonic, $t_{0}$ - the mean epoch of observations, $f_{0}, f_{1}, a_{k}$ and $b_{k}$ are unknown coefficients and $n$ is the number of harmonics of the partial sum, which covers all oscillations with periods between $P_{0} / n$ and $P_{0}$. The application of the LS estimation of Fourier coefficients needs at least $2 n+2$ observations, so the number of harmonics $n$ is chosen significantly smaller than the number $N$ of sampled data $f_{i}$. The small number of harmonics $n$ yields to LS estimation of the coefficient errors. This estimation is the first essential difference with the classical Fourier approximation. The second difference is the arbitrary choice of the period of first harmonic $P_{0}$, instead of the observational time span, so the estimated frequencies may cover the desired set of real oscillations. This method allows a flexible and easy separation of harmonic oscillations into different frequency bands by the formula

$$
B(t)=\sum_{k=m_{1}}^{m_{2}} a_{k} \sin k \frac{2 \pi}{P_{0}}\left(t-t_{0}\right)+b_{k} \cos k \frac{2 \pi}{P_{0}}\left(t-t_{0}\right),
$$

where the desired frequencies $\omega_{k}$ are limited by the bandwidth

$$
\frac{2 \pi m_{1}}{P_{0}} \leq \omega_{k} \leq \frac{2 \pi m_{2}}{P_{0}},
$$

After estimating the Fourier coefficients, it is possible to identify a narrow frequency zone presenting significant amplitude, and defining a given cycle. Then this cycle can be reconstructed in time domain as the partial sum limited to the corresponding frequency bandwidth. Doing this for terrestrial and solar time series, we shall identify their respective cycles, isolate and compare the common ones.

This method provides Least Squares estimate of 150 harmonically coefficients with average accuracy of about $8 \mathrm{~mW} / \mathrm{m}^{2}$ for TSI; and 0.002 for NAO.

\section{Results}

\section{a) Time series spectra}

The time series spectra of NAO and TSI are determined by the Fast Fourier Transform. The NAO and TSI spectra are almost coherent for periods between 12 and 200 years, so it is possible to find a lot of narrow frequency bands with common decadal and centennial TSI and NAO cycles. 


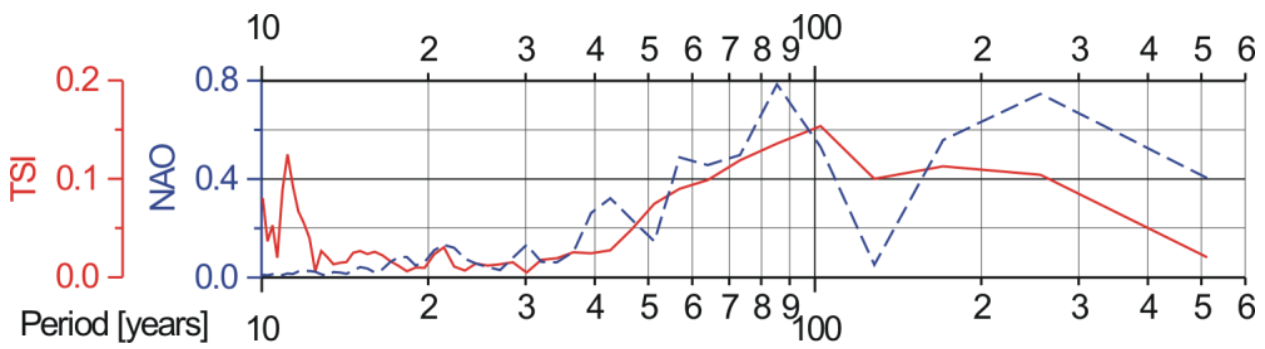

Fig.2. Spectra of NAO and TSI time series, determined by the FFT.

\section{b) TSI influence on NAO cycles}

The TSI influence on NAO variations is detected in 12 narrow frequency bands with interannual and decadal cycles with periods below 50 years (Figs. 3-6). These frequency bands are divided into 3 groups with periodicities 30 - 50yr; 20 - 30yr; and 8-20yr. The common TSI and NAO cycles from the first group of periodicities are shown in Fig.3. Excellent agreement exists between TSI and NAO cycles with periods 42.6 - 47.9 years (Fig.3, a), where is the essential 45-year solar cycle, associated with the N-S solar asymmetry. The other two bands expose relatively good agreement between TSI and NAO variations, where a phase reverse occurs in 1680-1770.

(a)

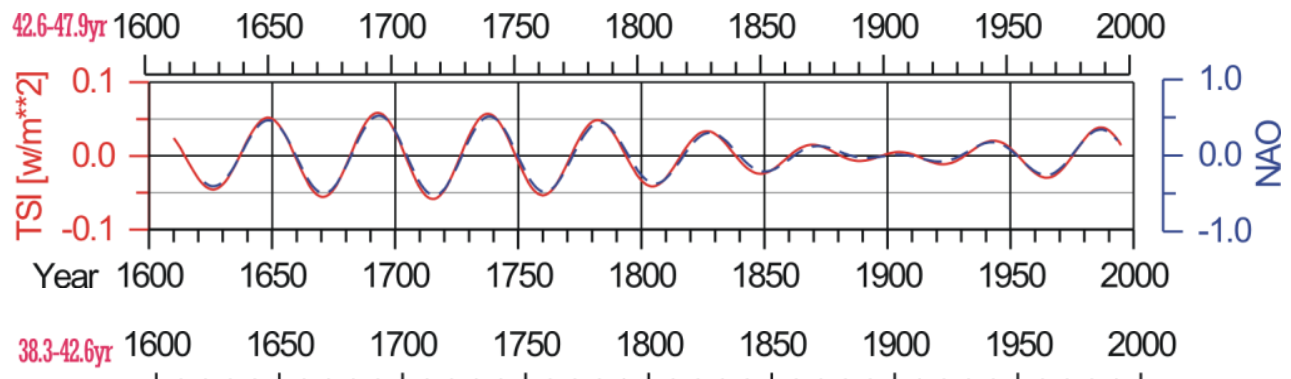

(b)
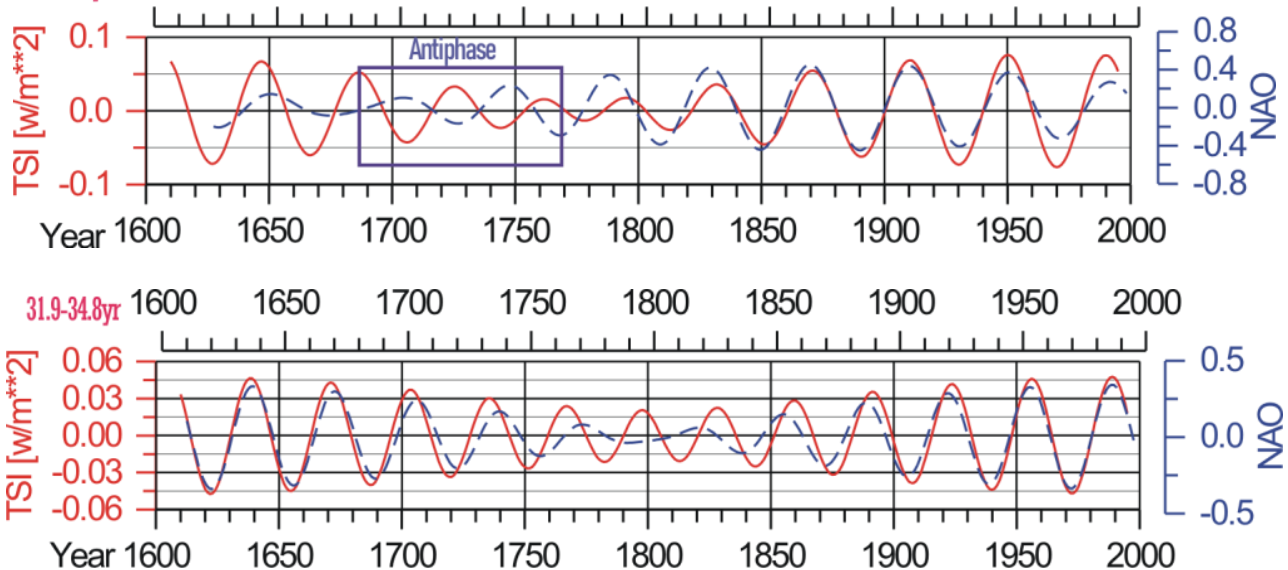

Fig.3. Common TSI and NAO cycles with periods from bands: 42.6 - 47.9 years (a); 38.3 - 42.6 years

(b); and 31.9 - 34.8 years (c).

The second group of frequency bands are presented in Fig.4. It covers periodicities between $29.5-31.9 ; 27.9-29.5$; and $25.5-27.4$ years. The agreement between the solar and climate cycles is relatively good, with two phase reverses. 
(a)

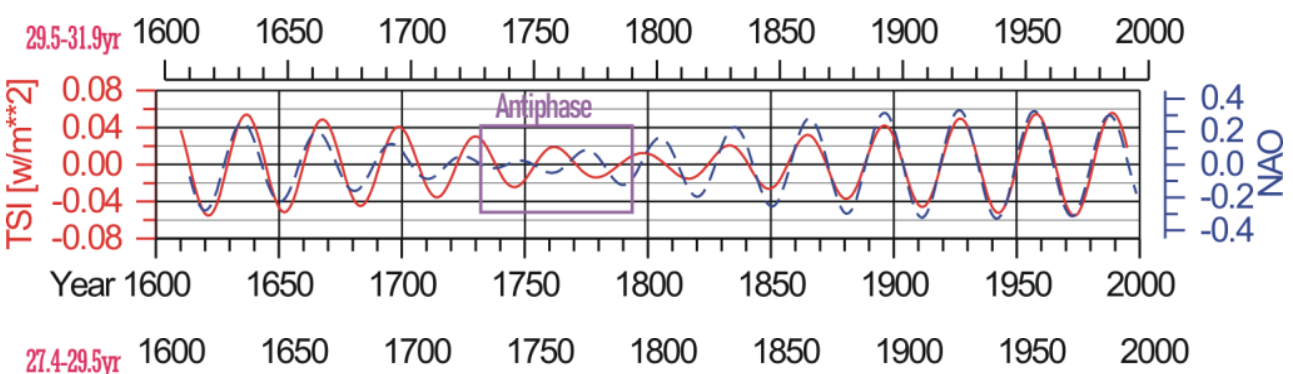

(b)

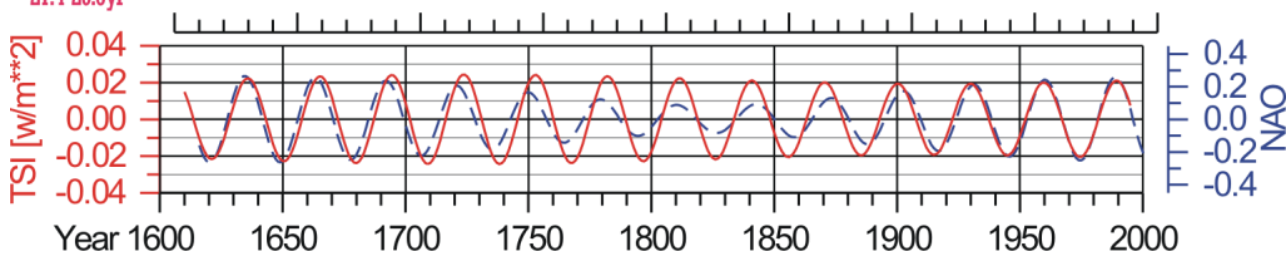

(c)

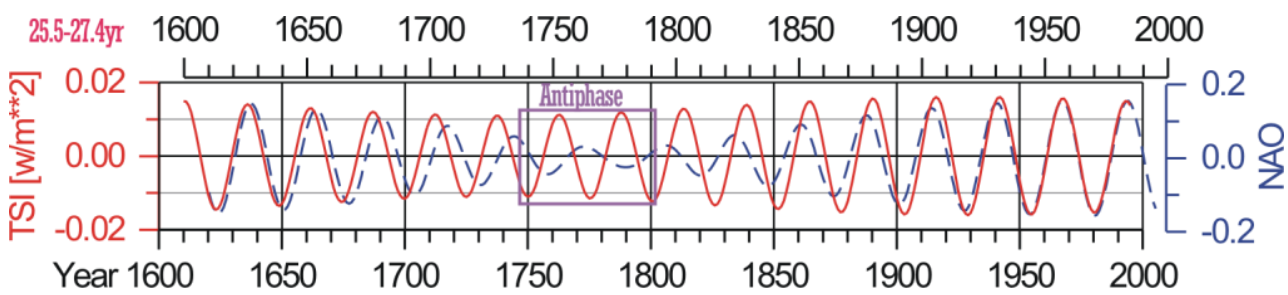

Fig.4. Common TSI and NAO cycles with periods from bands: 29.5 - 31.9 years (a); 27.9 - 29.5 years

(b); and $25.5-27.4$ years $(c)$.

The third group of TSI-NAO periodicities consists of 6 frequency bands, shown in Figs. 5 and 6. These frequency bands cover periodicities between $16.7-17.4 ; 14.2-14.7 ; 13.2-13.7$, 12.4 - 12.8; 10.6 - 11.2; and $8.5-8.7$ years. The TSI and NAO oscillations have common cycles with very good agreement for these periodicities with exception of 11-year Schwabe cycles, whose oscillations agree in 1850-2000 only (Fig.6, b).

(a)

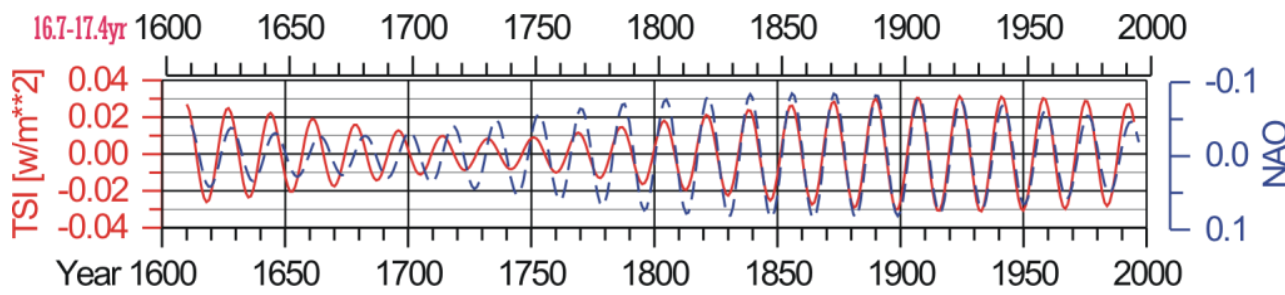

14.2-14.7yr $\begin{array}{lllllllll}1600 & 1650 & 1700 & 1750 & 1800 & 1850 & 1900 & 1950 & 2000\end{array}$

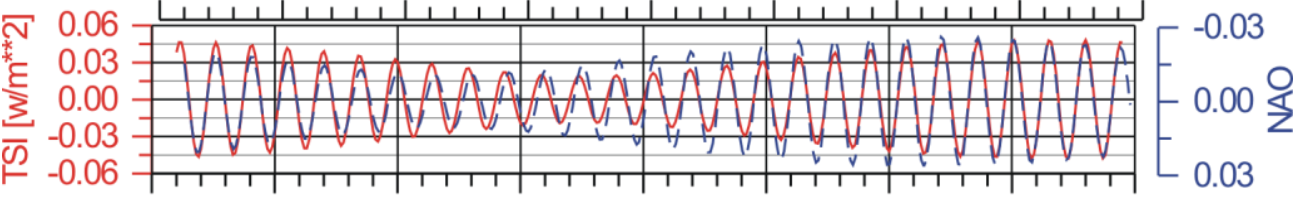

$\begin{array}{lllllllll}\text { Year } 1600 & 1650 & 1700 & 1750 & 1800 & 1850 & 1900 & 1950 & 2000\end{array}$

13.2-13.7y: $1600 \quad 1650 \quad 1700 \quad 1750 \quad 1800 \quad 1850 \quad 1900 \quad 1950 \quad 2000$

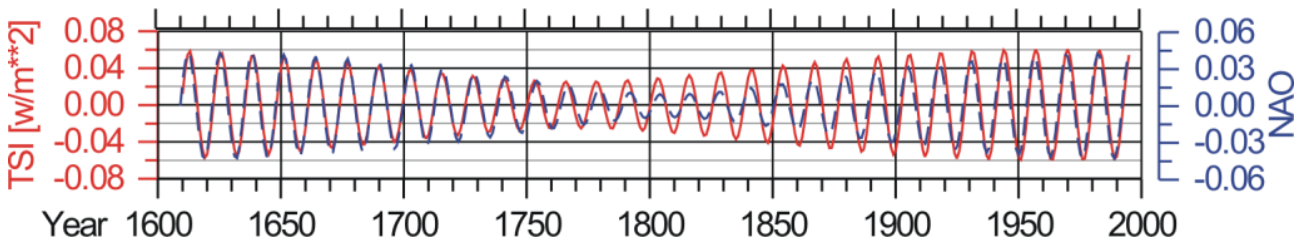

Fig.5. Common TSI and NAO cycles with periods from bands: 16.7 - 17.4 years (a); 14.2 - 14.7 years (b); and $13.2-13.7$ years (c). 
(a)

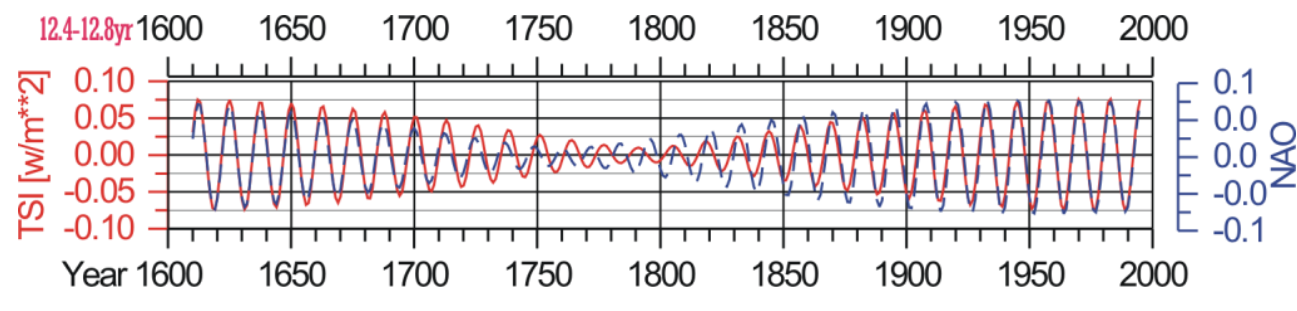

(b)

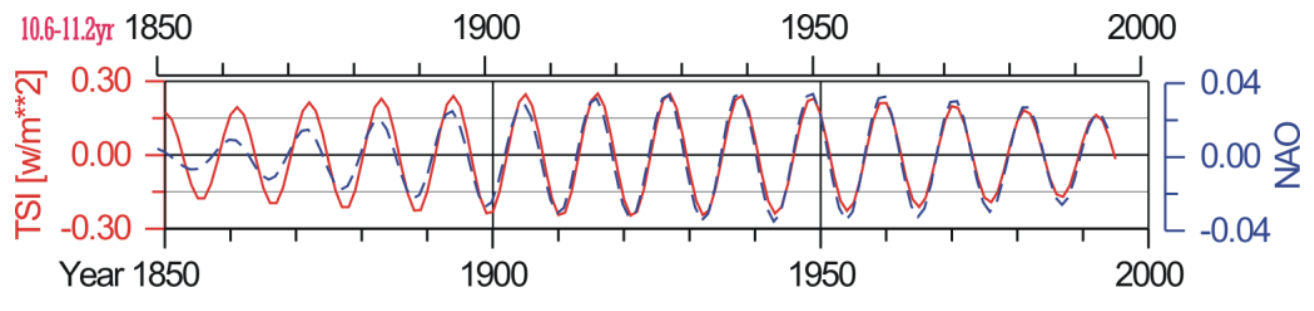

(c)

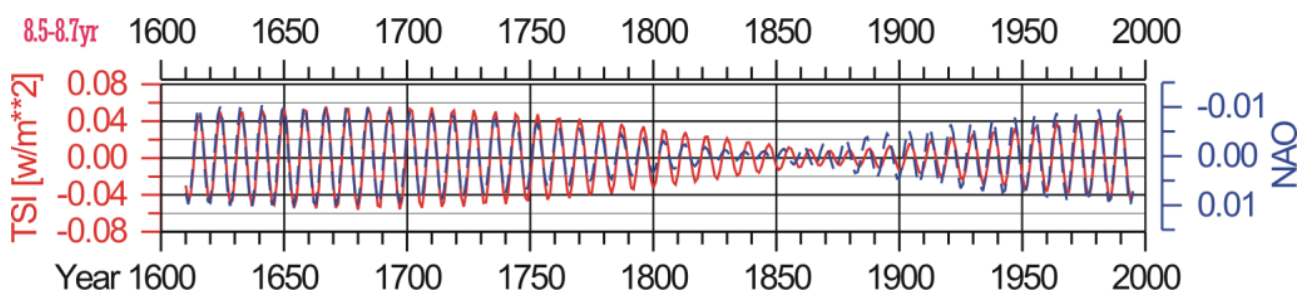

Fig.6. Common TSI and NAO cycles with periods from bands: $12.4-12.8$ years (a); $10.6-11.2$ years (b); and $8.5-8.7$ years $(c)$.

\section{Conclusions}

The common TSI and NAO cycles are determined by the Method of Partial Fourier Approximation. This method provides Least Squares estimate of the harmonically coefficients with average accuracy around $8 \mathrm{~mW} / \mathrm{m}^{2}$ for TSI; and 0.002 for NAO. The common cycles are shown in Figs. 3-6, where the solid lines represent TSI oscillations; and the dashed lines represent the NAO cycles. Very good agreement between TSI and NAO cycles exists in 12 interannual and decadal frequency bands. The common decadal oscillations are represented in bands with decadal periods 42.6 - 47.9yr, 38.3 - 42.6yr, 31.9 - 34.8yr, 29.5 - 31.9yr; $27.9-$ $29.5 \mathrm{yr}, 25.5-34.8 \mathrm{yr} ; 16.7-17.4 \mathrm{yr}, 14.2-14.7 \mathrm{yr}, 13.2-13.7 \mathrm{yr}$ and 12.4-12.8yr. The common interannual cycles are determine in a single band with periods $8.5-8.7 \mathrm{yr}$. The most of these common cycles have positive correlation and 3 of them - negative correlation.

The 11-year Schwabe oscillations are presented in frequency band with periods 10.6-11.2yr. Good agreement between 11-year TSI and NAO cycles exists in time interval 1850-1995 only (Fig.6, b). Before 1850 the TSI and NAO oscillation have different time lag and significant discrepancy of amplitude variations, so these are possible reasons of negative results about solarclimate interconnection in (van Oldenborgh et al., 2013)

The NAO is the dominant mode of winter climate variability in the North Atlantic region ranging from central North America to Europe and much into Northern Asia. The knowledge of common solar and NAO cycles may help better understanding of winter climate variability in North America and Europe. On the base of existing deep solar minimum, it is possible to predict high positive values of NAO index in next decade and corresponding windstorms and wet winters in Europe.

\section{Acknowledgements}

The study is supported by the National Science Fund of Bulgaria, Contract KP-06-N34/1 "Natural and anthropogenic factors of climate change - analyzes of global and local periodical components and long-term forecasts" 


\section{References}

Coddington, O., Lean, J.L., Pilewskie, P., et al. A solar irradiance climate data record // Bull. American Meteorological Soc. 97(7), 1265-1282, 2016.

Gorshkov V. and Y. Chapanov, Winter North Atlantic Oscillations Driven by Variations in Total Solar Irradiance, Geomagnetism and Aeronomy, Springer, 2020 (Accepted).

Trouet, V., J. Esper, N.E. Graham, A. Baker, J.D. Scourse, and D.C. Frank, Persistent Positive North Atlantic Oscillation Mode Dominated the Medieval Climate Anomaly, Science, Vol. 324, 78-80, 2009, DOI: 10.1126/science.1166349

van Oldenborgh, G.J., de Laat, A.T.J., Luterbacher, J., Ingram, W.J., and Osborn, T.J., Claim of solar influence is on thin ice: are 11-year cycle solar minima associated with severe winters in Europe?, Environ. Res. Lett., 2013, vol. 8, no. 2, 024014. doi 10.1088/1748-9326/8/2/024014 


\title{
Solar Influence on Seismic Energy
}

\author{
Chapanov Ya. ${ }^{1}$, Ron $C^{2}$, Vondrák $J^{2}$ \\ ${ }^{1}$ Climate, Atmosphere and Water Research Institute, Bulgarian Academy of Sciences, Sofia, \\ Bulgaria \\ E-mail: chapanov@gmail.com \\ ${ }^{2}$ Astronomical Institute, Czech Academy of Sciences, Boční II 1401, 14100 Prague, Czech \\ Republic
}

\begin{abstract}
.
The solar activity affects all surface geosystems, including weather and climate indices, winds, rains, snow covers, mean sea level, river streamflows and other hydrological cycles. The Total Solar Irradiance (TSI) acts directly on Earth systems variations, while the solar wind affects the variations of geomagnetic field. The solar magnetic field and solar wind determine the properties of the heliosphere up to the heliopause. The variations of geomagnetic field and heliosphere have ability to modulate the charged particles of the cosmic rays. The recent models of ozone production by the cosmic rays in upper atmosphere and its influence on water content, which is the most powerful greenhouse gas, point out to significant effects on local and global temperature and climate processes. The variations of winds, air pressure, rains, snow covers, mean sea level and other hydrological cycles, produce small local and global crust and mantle deformations. These deformations may trigger earthquake and volcanic seismicity with synchronous periodicity of the cosmic ray and solar activity. The solar influence on seismicity is investigated by common cycles of cosmic rays, solar, and Earth data. The solar data consist of centennial time series of TSI and North-South solar asymmetry. The Earth data are represented by geomagnetic index AA since 1870 and time series of earthquakes since 1898 . Relatively good agreement exists between long-term variations of seismicity, cosmic ray, solar and geomagnetic indices.
\end{abstract}

\section{Introduction}

The earthquakes and volcanic eruptions are the results of material circulation in the Earth that appear near the Earth's surface. Volcanoes and earthquakes have a lot in common. They both occur mostly near to the boundaries of tectonic plates. They have potential to cause significant damage. Volcanic eruptions are caused by the tectonic plates shifting and that can cause earthquakes. The seismic activities cause frequent natural disasters in many Earth regions. It is necessary to study not only earthquakes themselves but also all processes affecting them to predict seismic activity. The solar and geomagnetic activity affect significantly seismic variations. Some identical cycles of cosmic rays, solar and geomagnetic indices on the one hand, and earthquake energy on the other hand, are presented in this work.

The solar activity affects terrestrial systems by means of direct radiation over Earth surface, influences charged particles of the solar wind, and the solar magnetic field. The solar wind directly affects Earth magnetic field, ionosphere and atmosphere. The variations of solar magnetic field modulate solar wind and cosmic rays in the frame of the solar system. The cosmic rays near Earth are modulated by Earth magnetic field variations, too. Recently, a new mechanism of climate variations, due to cosmic rays was proposed (Kilifarska and Haight, 2005; Kilifarska, 2008, 2011; Velinov et al., 2005). According to the new models, the cosmic rays produce an ionization of the atmosphere, changes of atmosphere conductivity, and lightning, and an increase of ozone concentration. This mechanism is based on chain processes near tropopause by ozone production, temperature variations, followed by vertical winds and water content change. The last step of this chain affects strongly surface temperature, because the atmospheric water is one of the most powerful greenhouse gas. This model provides an 
explanation for the cascade processes in which cosmic rays, whose total energy is relatively small, cause climatic effects with much more energy. The solar activity cycles modulate the cosmic rays directly by the heliosphere and indirectly by the geomagnetic field changes, whose effect is visible mainly at high latitudes.

We suppose that the solar and space effects on climate variations produce different local deformation of the earth crust and they trigger earthquakes with a certain periodicity, equivalent for the whole seismic events. These periodic variations of seismicity are possible to be determined as seismic energy oscillations, whose cycles are identical with the cycles of cosmic rays, solar and geomagnetic variations. Assuming that the time series of seismic energy variations consist of artificial and natural linear trends and jumps, induced periodic cycles and instrumental random noise, we may separate these components and investigate them.

\section{Data}

The data consist of centennial time series of total solar irradiance, North-South (N-S) solar asymmetry, cosmic rays, geomagnetic index AA, earthquake numbers and magnitudes. The earthquake magnitudes are transformed into periodic variations of energy magnitude.

The TSI and N-S solar asymmetry time series are presented in Fig.1. The last version of estimated solar irradiances for the last 400 years is based on the NRLTSI2 historical TSI reconstruction model by J. Lean (Coddington et al., 2015). The N-S solar asymmetry (N-S SA, Fig. 1) is determined from the relation $\left(S_{n}-S_{s}\right) /\left(S_{n}+S_{s}\right)$, where the $S_{n}$ and $S_{s}$ are areas of sunspots on the Northern and Southern solar hemispheres, respectively.
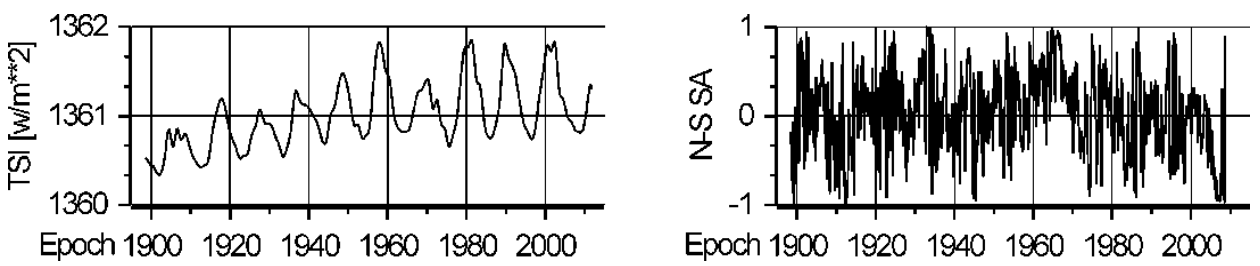

Fig.1. TSI and N-S solar asymmetry time series for the period 1898-.2012.

Usoskin calculates the intensity of galactic cosmic rays (CR) at the Earth's orbit since 1610 (Usoskin, 2002, 2005), see Fig. 2, left. The geomagnetic Index AA since 1868 is available at the International Service of Geomagnetic Indices. The mean variations of AA are determined by average in 27-day window (Fig. 2, right).
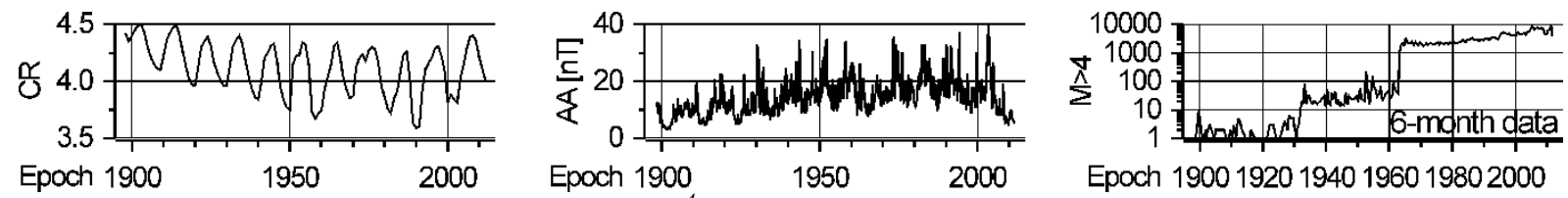

Fig. 2. Time series of Cosmic Rays $C R\left(\times 10^{4}\right.$ counts/hour $)$, geomagnetic Index AA and earthquakes number with magnitude $M>4$.

The number of registered earthquakes significantly vary in time (Fig.2, right panel). Initially, prior to 1930, limited number of short distance earthquakes have been registered, due to insufficient number of seismic stations with low level of sensitivity. The earthquake registration was improved significantly twice - in 1932-1962 and after 1963 by network modernization and involve of new station with higher sensitivity. The earthquake number slightly increase in all three time intervals, but it is difficult to separate effects of network improvement from natural reasons. So, the investigation of whole earthquake data since 1898.5 as a homogenous time series is not possible.

\section{Methods}

A new Method of time series determination of uniformly distributed seismic energy, with some homogeneity of oscillations at given frequency and amplitude variations, is proposed and 
applied here. This Method is based on excluding the piece-wise linear trends from the time series. The released energy $E$ during the earthquake with magnitude $M$ is generally

$E=K 10^{1.5 M}$,

where $K$ is dimension coefficient in Joules. Then the magnitude $M$ is

$M=\frac{1}{1.5} \lg \frac{E}{K}$

The global seismic energy release $E_{0.1}$ at 0.1 -year time interval is a sum of energy from all seismic events in this interval

$E_{0.1}=K \sum_{t \in 0.1 y r} 10^{1.5 M(t)}$.

Let introduce $E_{M}$ - magnitude of the global seismic energy release at $0.1 \mathrm{yr}$. Then we may determine $E_{M}$ analogous to (2)

$E_{M}=\frac{1}{1.5} \lg \frac{E_{0.1}}{K}$

or calculate $E_{M}$ by the expression

$E_{M}=\frac{1}{1.5} \lg \sum_{t \in 0.1 y r} 10^{1.5 M(t)}$.

The time series of calculated seismic energy magnitude are given in Fig. 4. The jumps and linear trends in time series of seismic energy are determined by the method of jump detection, described in (Chapanov et al., 2017). This method is highly sensitive to very small data jumps, because it transforms the initial data to a new series by numerical integration. Any data jump is detectable as linear break in integrated time series, where the linear trends of original data are expressed by parabola, while the constant data variations - by straight lines. The corresponding parts with detected trends and jumps of integrated and original data are marked with red and blue color in Fig. 4. There are 6 parts with linear trends and one constant part in original data, separated by jumps in 1918.5, 1930.0, 1934.0, 1957.0, 1963.0 and 1995.0 (Fig. 4). The detrended time series of energy magnitude are shown in Fig. 5. After the trend remove, the new time series contain periodic variations and random noise, whose value is relatively high in 19281958.
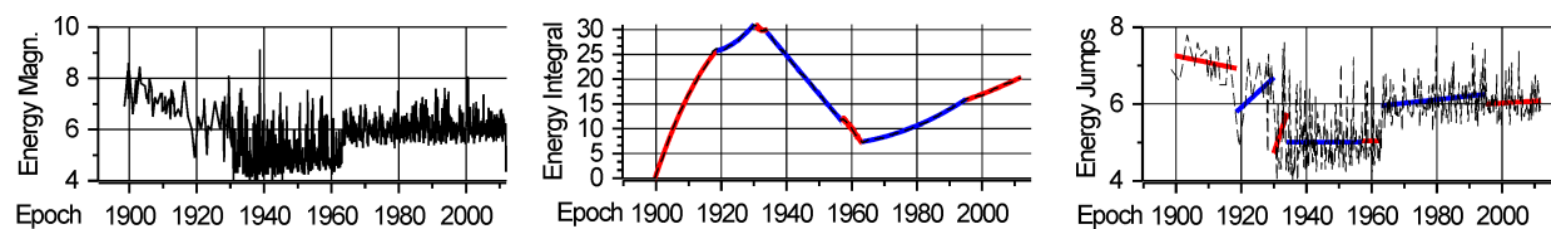

Fig. 4. Determination of linear trends in time series of energy magnitude by the method of jump detection.

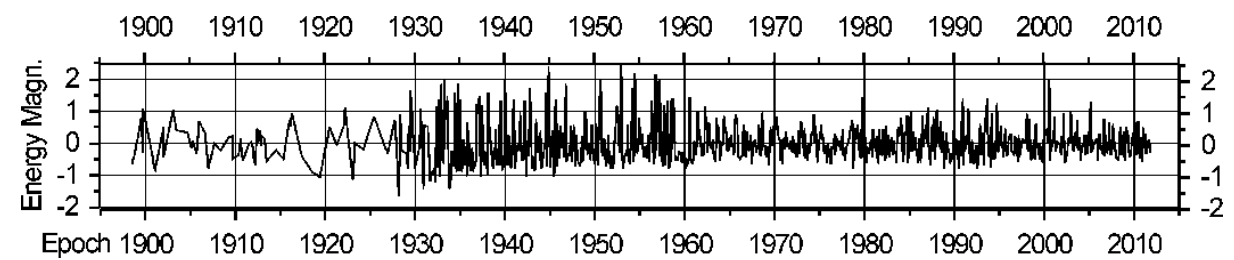

Fig. 5. Periodic variations and random noise in detrended time series of energy magnitude.

The time series of oscillations from a given frequency band are calculated as a superposition of two neighbor Fourier harmonics, whose coefficients are estimated by the Least Squares Method inside the Method of Partial Fourier Approximation (PFA). The details of the last method are described in (Chapanov et al., 2015). 


\section{Solar influence on earthquakes}

All solar and space factors influence seismic activity indirectly. The prime effect of earthquakes is supposed the local crust deformations nearby the faults. The local crust deformations are due to the change of ocean and atmosphere load, precipitation and ground water content, tides, snow and ice shit thickness variations. The solar heat, represented by the TSI, and cosmic rays influence directly the climatic effects on crust deformation. The solar wind and solar magnetic field modulate and form solar plasma inside the whole heliosphere. The interconnection between the cosmic rays and heliosphere modulates the intensity of incoming particles of the cosmic rays. The geomagnetic field, affected by the variations of solar wind and N-S solar asymmetry, plays a role of last focussing lens of incoming charged cosmic rays.

\section{Earthquake energy variations due to cosmic rays}

The cosmic rays have common subdecadal cycles with earthquake energy variations with periods between 3.3-3.4yr; 4.7-4.9yr and 6.3-6.7yr. These cycles have similar phase and amplitude variations with small time delay as can be seen at Fig. 7. The 20-year shift between the minima of CR and $E_{M}$ amplitudes in case of oscillations with 3- and 4-year periods means that these oscillations are disturbed by TSI variations, too. So, the best pattern of CR influences on earthquake energy are 6.3-6.7 cycles.
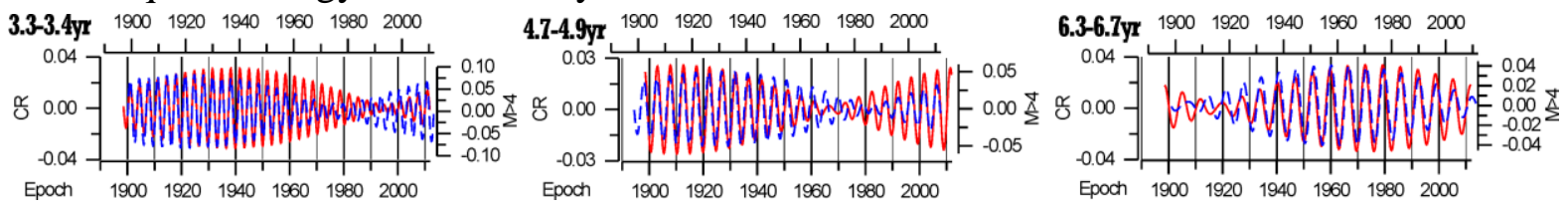

Fig. 7. Common oscillations of cosmic rays CR (solid red line) and earthquake energy (dashed blue line) with periods 3.3-3.4; 4.7-4.9 and 6.3-6.7 years.

\section{Earthquake energy variations due to TSI cycles}

The Fig. 8 shows common cycles of TSI and earthquake energy from different frequency bands. Almost exact match exists between TSI and earthquake energy cycles with periods 7.58.1, 12.6-14.2 and 16.2-18.9 years. The cycles with periods 6.6-7.1 and 8.7-9.4 years have very good agreement. The 14.2-16.2yr cycles are affected by phase reverse during 1930-1960. The subdecadal oscillations with periods 4.9-5.1 are affected by the CR variations, too.

\section{Earthquake energy variations due to solar and terrestrial magnetic fields}

The long term variations of earthquake energy are governed by the solar and terrestrial magnetic fields variations. In Fig. 9 and 10 these variations are represented by indices N-S SA and AA. Their variations cover all long periodical oscillations of earthquake energy between 28 and 113 years with a significant minimum in 1990-2000, and clear increase after that. The negative correlation coefficient between AA and $E_{M}$ variations is a signature of CR influence, too. 

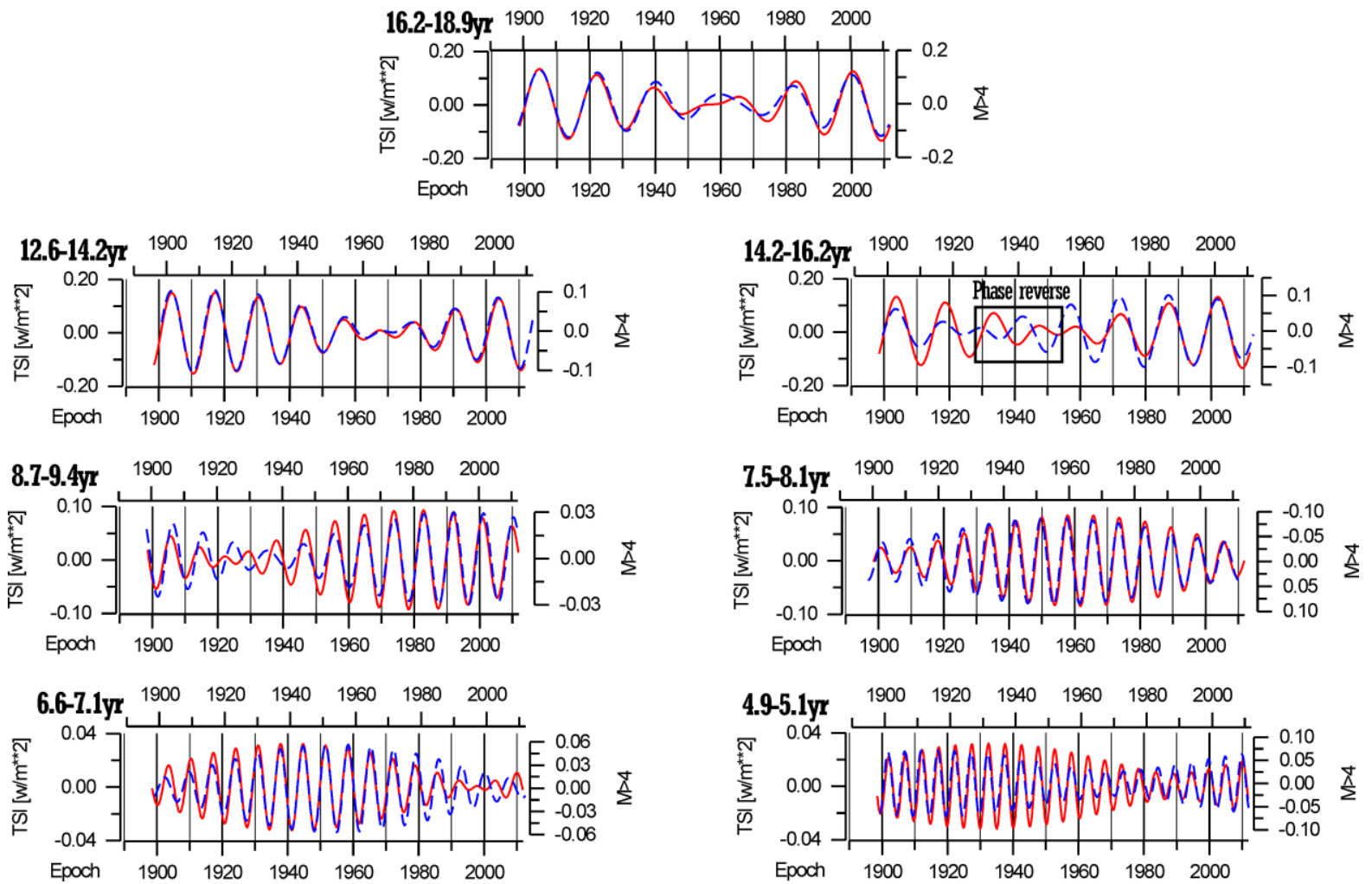

Fig. 8. Common cycles of TSI (solid red line) and earthquake energy (dashed blue line) with periods

4.9-5.1; 6.6-7.1; 7.5-8.1; 8.7-9.4; 12.6-14.2; 14.2-16.2 and 16.2-18.9 years. The curves with negative coefficient of correlation are drawn with reversed $y$-axis.
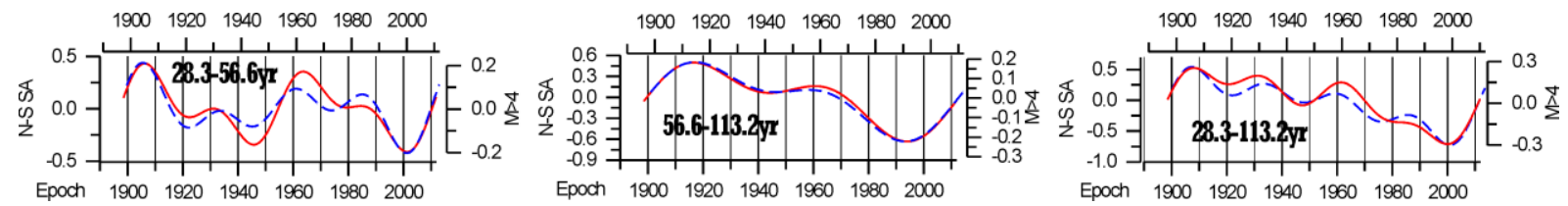

Fig. 9. Long term variations of earthquake energy (dashed blue line) due to $N$-S solar asymmetry

(solid red line).
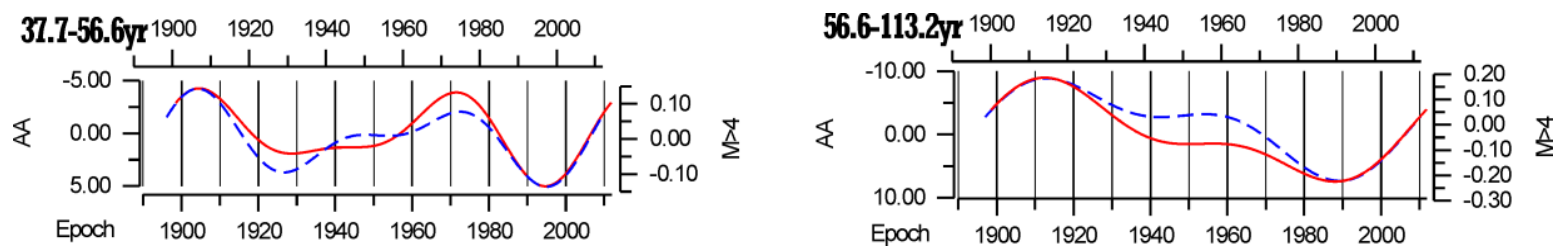

Fig.10. Long term variations of earthquake energy (dashed blue line) due to geomagnetic field

oscillations (solid red line). The curves with negative coefficient of correlation are drawn with

reversed $y$-axis.

\section{Conclusions}

The shapes of decadal and centennial solar cycles are rather different from sinusoidal form, and this is the reason to generate a lot of subdecadal and decadal harmonics. The solar activity affects all geosystems, including climate and weather. The TSI cycles are the main source of climate indices variations. The solar wind and solar magnetic field form heliosphere plasma, whose interaction with the galactic cosmic rays modulates their intensity. The solar wind modulates the shape of geomagnetic field, which plays a role of focusing lens of the falling cosmic rays. The cosmic rays produce a chain process near tropopause by ozone production, temperature variations, followed by vertical winds and water content change. The last step of this chain strongly affects surface temperature, because the atmospheric water is one of the most powerful greenhouse gas. The cosmic rays, whose total energy is relatively small, cause 
climatic effects with much more energy by this cascade process. The climate cycles, induced by direct and indirect effects of solar activity, produce local crustal deformations, that are supposed to trigger seismic activity. The TSI and N-S solar asymmetry have individual spectra in some frequency bands, so they affect differently climate and seismic variations.

The registered earthquakes since 1898.5 are not regular in time and it is difficult to analyze these centennial time series. Supposing that the periodical components of earthquake energy variations depend on intensity and frequency of external forces, we remove all significant linear trends from the time series of earthquake global energy release at 0.1-year steps. The transformed time series consist of random noise and periodical component, synchronized with the oscillations of external forces, and they have tidal, solar and space origin. The individual analyzes of solar and space influences on earthquake energy variations show that the TSI variations have major influence on seismic oscillations. The TSI harmonics with periods between 5 and 20 years affect seismic energy by identical cycles from several frequency bands. The long-term variations of seismic energy are affected by N-S solar asymmetry and geomagnetic field modulation with periodicities between 28 and 113 years. The cosmic ray harmonics affect seismic energy variations with periods below 7 years.

\section{Acknowledgements:}

This research was financially supported by the project RVO:67985815 provided by the Czech Academy of Sciences; and by the National Science Fund of Bulgaria, Contract KP-06N34/1 "Natural and anthropogenic factors of climate change - analyzes of global and local periodical components and long-term forecasts".

\section{References}

Chapanov, Ya., Ron C. and Vondrák, J.: 2015, Millennial cycles of mean sea level excited by Earth's orbital variations. Acta Geodyn. Geomater., 12, 3 (179), 259-266. DOI: 10.13168/AGG.2015.0028

Chapanov, Ya., Ron, C. and Vondrák, J.: 2017, Accuracy and sensitivity of a method of impulse detection, evaluated by simulated time series. Acta Geodyn. Geomater., 14, 1 (185), 73-82, DOI:10.13168/AGG.2016.0029.

Coddington, O., Lean, J.L., Pilewskie, P., Snow, M. and Lindholm, D.: 2015, A solar irradiance climate data record, Bull. American Meteorological Soc. doi: 10.1175/BAMS-D-14-00265.1

Hempel, L., and F. Thyssen. 1992. Deep radio echo soundings in the vicinity of GRIP and GISP2 drill sites, Greenland. Polarforschung 62:11-16.

Kilifarska, N.A. and Haight, J.D.: 2005, The impact of solar variability on the middle atmosphere in present day and pre-industrial atmospheres. J. Atmos. Solar Terr. Phys., 67, 3, 241-249. DOI: 10.1016/j.jastp.2004.10.003

Kilifarska, N.A., Tassev, Y.K. and Tomova, D.Y.: 2008, Cosmic ray showers and their relation to the stratospheric sudden warmings. Sun and Geosphere, 3, 1, 10-17.

Kilifarska, N.A.: 2011, Long -term variations in the stratospheric winter time ozone variability - 22-year cycle. Comptes rendus de l'Académie bulgare des Sciences, 64, 6, 867-874.

Palais, J.M., Germani, M.S. and Zielinski, G.A.: 1992, Interhemispheric transport of volcanic ash from a 1259 A.D. volcanic eruption to the Greenland and Antarctic ice sheets. Geophysical Research Letters 19:801-804. doi:10.1029/92GL00240

Palais, J.M., Taylor, K.C., Mayewski, P.A. and Grootes, P.M.: 1991, Volcanic ash from the 1362 A.D. Oraefajokull eruption (Iceland) in the Greenland ice sheet. Geophysical Research Letters 18:1241-1244, DOI: 10.1029/91GL01557

Usoskin, I.G., Mursula, K., Solanki, S.K., Schüssler, M. and Kovaltsov, G.A.: 2002. A physical reconstruction of cosmic ray intensity since 1610. J. Geophys. Res., 107(A11), 1374. doi:10.1029/2002JA009343

Usoskin, I.G., Alanko-Huotari, K., Kovaltsov, G.A. and Mursula, K.: 2005, Heliospheric modulation of cosmic rays: Monthly reconstruction for 1951-2004. J. Geophys. Res., 110(A12), A12108. doi:10.1029/2005JA011250

Velinov, P.I.Y., Mateev L. and Kilifarska N.A.: 2005, 3-D model for cosmic ray planetary ionisation in the middle atmosphere. Ann. Geophys., 23, 9, 3043-3046. DOI: 10.5194/angeo-23-3043-2005

Wills, R. C. J., Armour, K., Battisti, D. S. and Hartmann, D. L.: 2019, Ocean-atmosphere dynamical coupling fundamental to the Atlantic multidecadal oscillation. J. Climate, 32, 1, 259-272. DOI:10.1175/JCLI-D-180269.1 


\title{
The Volcanic and Solar Activity Relationship During the Last 460 Years. Could a Significant Part of the "Sun-Climate" Relationship Goes Through Lithosphere?
}

\author{
Komitov B. ${ }^{1}$, Kaftan $V .^{2}$ \\ ${ }^{1}$ Institute of Astronomy and NAO - Bulgarian Academy of Sciences \\ ${ }^{2}$ Geophysical Center - Russian Academy of Sciences
}

\begin{abstract}
The volcanic activity behavior and the comparison of the last one to the solar activity during the interval 1550-2014 AD is the subject of present study. For this aim two databases have been used: 1. The Global volcanism program data base of Smithsonian Institute- Museum of natural history; 2 . The extended time series of solar activity indices of Pulkovo observatory. A statistically certain cycles by duration of 11, 19-26, 50- 60, 80-90 and 240-250 years in the annual new-generated volcanic eruption number time series has been established. There are analogues in the solar and the geomagnetic activity. It has been also found that there is a tendency of concentration of the moderate and strong volcanic events (volcanic eruptive index $\mathrm{VEI} \geq 4$ ) around the solar Schwabe-Wolf's cycles extrema. This tendency is very strong for the most powerful volcanic events (VEI $\geq 6$ ). Trigger mechanisms of solar and geomagnetic activity over volcanic events as well as their relation over climate changes (in interaction by galactic cosmic rays (GCR) and/or solar energetic particles (SEP)) are briefly discussed.
\end{abstract}

\section{Introduction}

The problem for the relationship between solar activity and tectonic events is discussed at least from the beginning of $20^{\text {th }}$ century. Good overviews of the first studies in this direction provided before $1920^{\text {th }}$ have been given by Tchijevskii (1976). There are a large number of studies concerning the solar and seismic activity relationships, but the corresponding number for solar-volcanic connections is too small. There are only sporadic papers, concerning the problem (see Stothers, 1989; Strestik, 2003). In summary the anticorrelation between the solar and volcanic activity is the most often suggested conclusion according these publications. As an additional independent argument supporting this conclusion is the "Danjon effect"- the relatively higher frequency of "dark" lunar total eclipses during the solar minima epochs (one to two years after the Schwabe-Wolf sunspot cycle minimum), which has been established by the French astronomer Andre-Louis Danjon about 100 years ago (Danjon, 1921). The dark solar eclipses events are in strong relationship with the volcanic dust concentration in Earth atmosphere. Evidences for presence of possible solar modulated long term volcanic activity cycles of centurial and supercenturial duration (80-90 and 200-215 years) are also given (Strestik,2003). It could say that there is a consensus between the researchers that the possible physical mechanisms of the solar volcanic activity relationship are by trigger-type nature.

As it has been pointed out by author and Stoychev (Komitov, 2011; Komitov and Stoychev, 2011) there are evidences that essential part of the strong volcanic eruptions (volcanic eruptive index VEI $\geq 5$ ) are connected no only to solar minima, but also occur during the sunspot maxima epochs.

This fact leads to many additional questions concerning the solar - volcanic activity relationship in different time scales and epochs as well as the possible physical mechanisms. The possible answers on some of them are the subject of the present work. 


\section{Data and methods}

In this study the volcanic eruption data, which are published in Smithsonian Museum of Natural History -Global Volcanism Program web site (https://volcano.si.edu) are used. On the base of the above mentioned data a relatively reliable scenario about volcanic activity approximately since 1550 AD could be build.
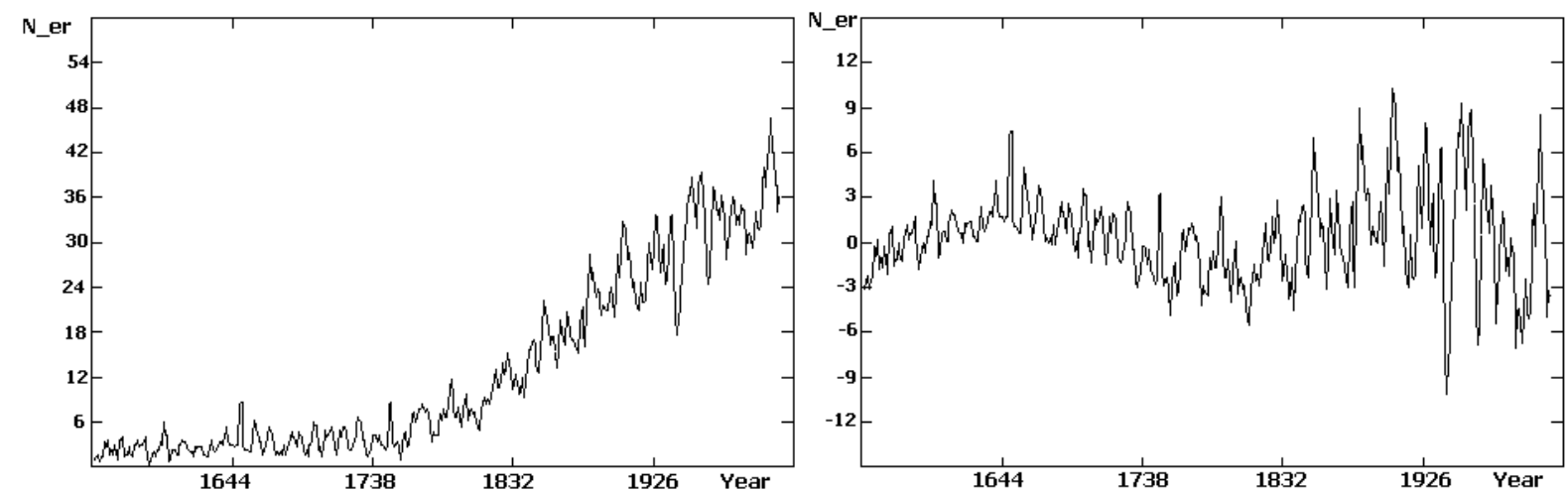

Fig. 1. Left: Smoothed 3-year annual number of volcanic eruptions time series (1550-2014 AD) Right panel ( $N \_$er): The "residual" series after removing of non-linear trend

According to the published data 6016 volcanic eruptions during the period 1550-2014 AD have been occur. Taking into account the trigger mechanism hypothesis a time series of annual numbers for the new generated (i.e. during the corresponding year) volcanic eruptions $\left(N_{-} e r\right)$ was build. Thus the volcanic events, which are starting in some previous calendar year, but are continuing during the corresponding year has been excluded from research process.

The so obtained time series of $N \_e r$ is shown on fig.1. A strong upward trend is visible there. With high confidence it could to suggest that the last one is caused first of all by lack of data during the earlier part of time series ( 1550-1750 AD), but some contribution of a real upward effect is too difficult to take or reject.

After removing of the trend over the "residual" time series a 3-year smoothing procedure has been provided. The smoothed time series has studied for existence of statistical significant cycles by using of two methods: the T-R periodogram algorithm (Komitov, 1986, 1997) and wavelet analysis (Torrence and Combo, 1998).

By using of histograms the absolute number and frequency of the strong volcanic eruptions (VEI $\geq 4$ ) in relation to Schwabe-Wolf's sunspot cycle phase are studied too. For this aim the data for the individual lengths of sunspot cycles during the epoch 1550-2014 AD has been used. It is based on the information from the Schove series (Schove, 1983) and extended sunspot instrumental series since 1705 AD. The obtained results and their analysis are described below.

\section{Results and analysis}

The T-R spectrum (correlogram) of smoothed VEI "residual" time series is shown on the left panel on fig.2. On the right panel of the same figure is the corresponding global wavelet spectrum. Statistically significant cycles by duration of 11, 19-25 (multiplet), 52.5- 61.5 (doublet) and 138 years as well as a very powerful 249.5-year cycle are visible in the T-R spectra. There is weak "trace" of $\sim 90 \mathrm{yr}$ cycle too. These results are in agreement with the global wavelet spectra where cycles buy duration of 11, 25, 60 and 240 years are shown.

There are many solar as well as other space climate analogues of almost all of detected cyclic oscillations in the "residual" VEI time series. The 11yr oscillation corresponds to the mean duration of Schwabe-Wolfs's sunspot activity cycle plus a large number of related to him solar and geophysical activity indexes and events such as solar flares, coronal mass ejections (CME), 
geomagnetic activity, galactic cosmic rays (GCR) etc. The $\sim 22$-year oscillation which is an important component in the quasi bi- decadal multiplet (19-25yr) corresponds to the Hale's solar magnetic dipole cycle. A cycle by duration of $\sim 60$ years has been established in middle latitude aurora activity (MLA) as well as in "cosmogenic" ${ }^{10} \mathrm{Be}$ isotope production by Komitov (2009), while the quasi 90 year variations corresponds to solar 88-90 year Gleissberg's cycle. (Gleissberg, 1944) Approximately 140 year cyclic variations have been detected in "cosmogenic" ${ }^{14} \mathrm{C}$ tree rings data series (Dergachev, 1994, 2004), which is close to $138 \mathrm{yr}$ cycle in T-R spectra on fig.2 (left panel).

The possible origin of quasi 250-year cycle in "residual" VEI will be separately discussed below in the text.
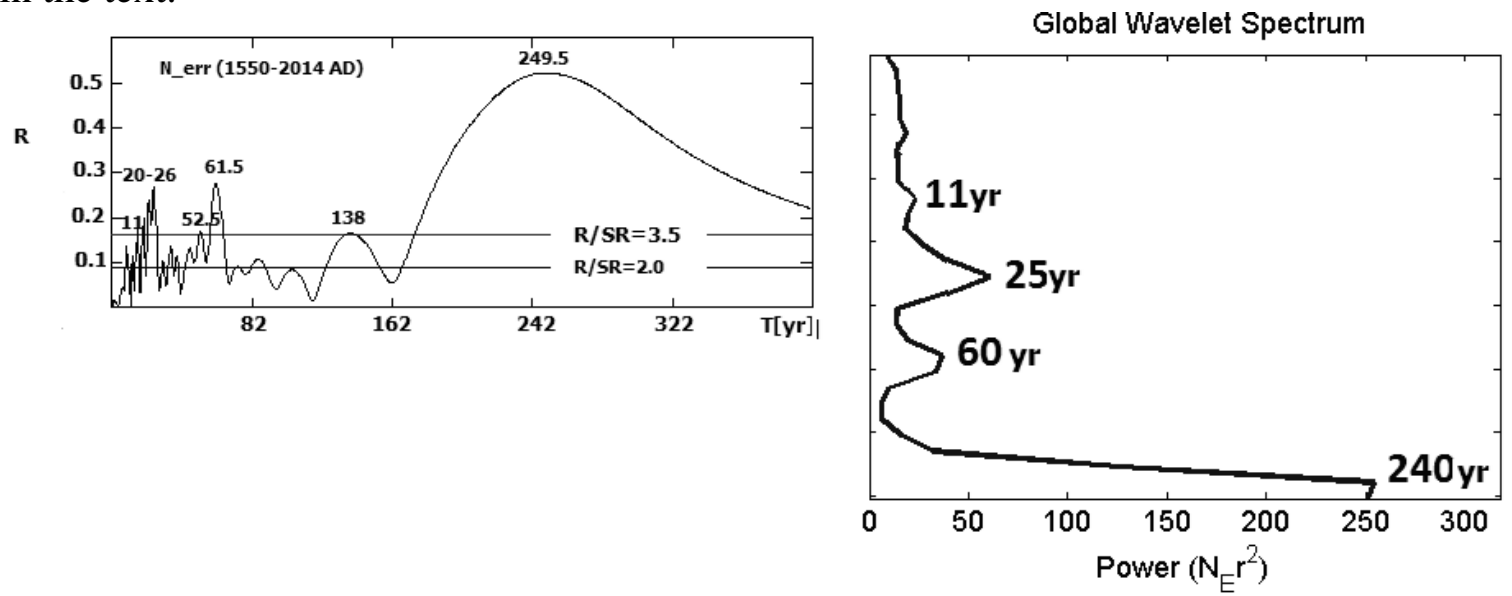

Fig.2 Left: The T-R spectrum of the "residual" volcanic eruptions time series; Right: Global wavelet spectrum of the same series

As it has been already pointed above the study of absolute number and/or frequency of strongest volcanic eruptions (VEI $\geq 4$ ) in relation to Schwabe-Wolf's sunspot cycle phase by using of histograms is the second main purpose of the present study.

The total number of the above mentioned volcanic eruptions during the interval 1550-2014 $\mathrm{AD}$ is 193. For each such event it has been calculated the current Schwabe-Wolf's sunspot cycle phase as $\Phi(t)=\frac{t-t_{0}}{L_{S W}}$, where $t$ is the current calendar year, $t_{0}$ and $L_{S W}$ are the calendar year of minimum and the length of current sunspot cycle correspondingly. The relative number of strong volcanic eruptions in percents for corresponding phase $\Phi$ in width intervals of 0.1 from 0 to 1 are shown in histogram on fig.3 (left-upper panel). Analogous relative number of strong volcanic eruptions in percents for corresponding phase $\Phi$ in width intervals of 0.1 from 0 to 1 are shown in histogram on fig.3 (left-upper panel). Analogous histograms of the volcanic eruptions, for which VEI is equal to 4 and 5 are shown on the right- upper and left-lower panels correspondingly .The histogram for the strongest volcanic eruptions (VEI $\geq 6$ ) (lower right panel) shows not the relative, but the absolute number of these events, because of their very small number- there are only 8 cases since 1550 AD.

As could be seen in these histograms there are two ranges of $\Phi$ were the strong volcanic eruptions most often occur - near to sunspot cycle minima (i.e. $0.9 \leq \Phi \leq 1$ and $0 \leq \Phi \leq 0.2$ ) and near to sunspot maxima $(0.3 \leq \Phi \leq 0.6)$. This is clear remarkable for the strongest eruptions (VEI=5 and VEI $\geq 6$ ), while including of the "moderate strong" eruptions (VEI=4) lead to some damaging of this tendency. On the other hand there is a permanent "hole" in all of these histograms $(0.2 \leq \Phi \leq 0.3)$ where the strong volcanic eruptions number is relative small or even equal to zero. 

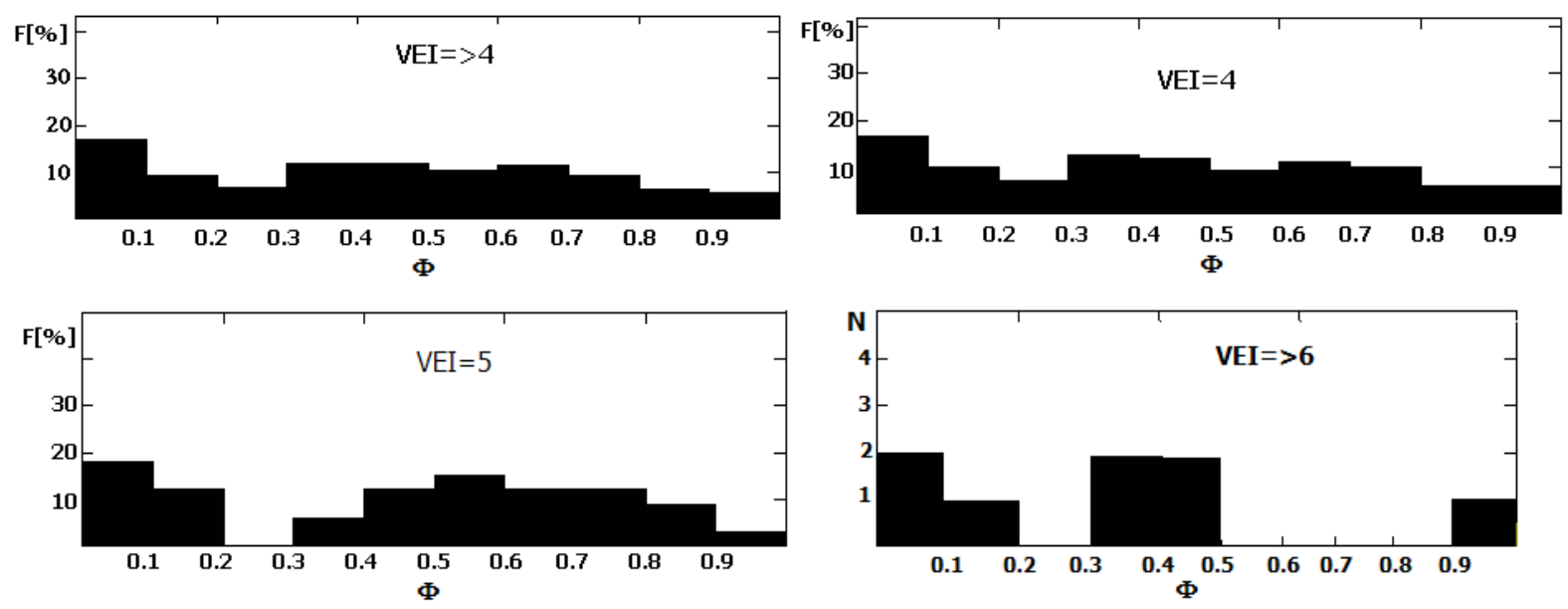

Fig. 3 The relative $(F \%)$ and absolute number $(N)$ of strong volcanic activity vs. sunspot activity phase $(\Phi)$ : (upper left: whole strong eruptions (VEI $\geq 4)$; upper right: moderate strong (VEI=4); very strong (VEI=5); strongest $(V E I \geq 6)$ )

\section{Discussion}

The problem for possible solar generated trigger mechanisms of strong volcanic events is of high importance in interdisciplinary scientific viewpoint at least by two reasons: 1 . It is interesting for the better understanding of conditions, when strong volcanic eruptions could occur with higher probability; 2. The important role of volcanic activity over Earth climate changes is out of doubt. Consequently, it is completely possible that an essential part of "Sunclimate "relation is caused not due to solar electromagnetic radiation changes, but rather by triggering due to solar activity processes over lithosphere.

That's why the physical nature of these trigger mechanisms indeed should be search on the course of solar flare activity and related to him space weather events - solar energetic particles, coronal mass ejections (CME's), geomagnetic storms, ground level enhancements (GLE) etc. Such events could affect different terrestrial electrical phenomena as the electrical potentials between ionosphere and earth surface changes, piezoelectric events in the upper lithosphere of continents, electrostatic interactions between electrical charges in the lower atmosphere and magma chambers. Thus the strong solar eruptive phenomena could be primary sources for triggering no only for volcanic, but also for seismic events as well as for some part of Earth rotation irregularities like the LOD (Length of Day) -index or changes or inner continental water bodies levels like Caspian Sea (Kaftan et al., 2018).

The double peaked volcanic eruptions histograms (fig.3) needs to suggest additional solar triggered mechanisms except of these, which are described above. It is necessary because due to the solar flare activity could explain the volcanic peak distribution near to the sunspot activity maxima, but not the second volcanic peaks near to sunspot activity minima. By our opinion such primary trigger mechanism could be the GCR flux. Due to the heliosphere parameters changes the last one is higher near to $11 \mathrm{yr}$ sunspots minima and lower to sunspots maxima.

The GCR-flux enhances during the sunspot minima lead to higher ion-electron concentrations in the lower troposphere. The last one remains in few orders lower related to ionosphere $\left(10^{-4}\right.$ to $10^{-2} \mathrm{~cm}^{-3}$ in troposphere vs. $10^{5} \mathrm{~cm}^{-3}$ to $10^{6} \mathrm{~cm}^{-3}$ in ionosphere), but this increasing could change serious the near surface atmosphere electric conductivity and as a result to significant changes of atmosphere-lithosphere electric potentials. The last one could produced a trigger effect to produce volcanic eruption if the magma chamber conditions approached to some critical limit.

Swensmark and Friiz-Christensen (Swensmark and Friiz-Christensen, 1997) and few years 
later $\mathrm{Yu}(\mathrm{Yu}, 2002)$ propose the idea that the enhanced ion-electron production in lower atmosphere during sunspot minima epochs could stimulated the aerosols and clouds origin and as a result - more precipitations and climate cooling should be observed in these epochs. As it was pointed by $\mathrm{Yu}$ (2002) the process should be essentially more effective if there are significant quantity of volcanic materials (dust and acid molecules $\mathrm{H}_{2} \mathrm{SO} 4, \mathrm{HCl}, \mathrm{HF}$ ) in the atmosphere.

The last circumstance is both very interesting and important. By the last one follows that an increasing of volcanic activity during the sunspot minima epochs leading to enhancements of dust and acid gases densities in atmosphere should be stimulate aerosols and clouds production much more effective. It is due to combination effect with the enhanced charge particles production by GCR in the troposphere at the same time. That's why "Danjon-effect" occur predominantly during the sunspot minima epoch. The GCR flux and the ionization rates in troposphere are relative low during the sunspot maxima in spite of possible higher volcanic eruptive activity.

Without of doubt the most famous result in our present study is the powerful 240-250-year cycle. By our opinion there are three hypotheses for its explanation. According to the first one it is related to specific solar activity regions like the giant (naked eye visible) sunspot groups. A 250-year cycle has been established for these sunspots on the base of Chinese catalog of eye visible sunspots (Vaguero et al., 1997). It is well corresponding to the solar triggering mechanism and seems to be most probable. According to the second hypothesis the 240-250 yr cycle of volcanic activity is caused by influence of induced magnetosphere of Venus, while according to the third one the primary source of this cycle is a tidal effect of Venus over the Earth lithosphere. The second and third hypotheses are less probable. It follows by these hypotheses that a 243-year cycle should be observed.

As an "extreme" example, which confirm the high probability of solar triggering hypothesis is the Pinatubo volcano activity in 1991 AD. The very close coincidences between the main separate phases of volcanic activation and solar flare activity and its accompanying space weather events are described in details by Komitov and Stoychev (2011).

Even so, it should to note again, that the "solar-volcanic activity" relationship is by triggered nature. It could only to increases the probability for strong solar eruption, while the main primary sources of volcanic activity are the inner magma chamber processes.

The evidences of solar-volcanic triggering could to consider as a base for new stage of the "Sun-climate" debate. Since the volcanic activity as a serious climate factor is considered it could to suggest that important part of whole "Sun-climate" relationship is masked by means of strong volcanic eruptions.

\section{Conclusions}

In this study evidences about solar activity trigger influence over the strong volcanic eruptions (VEI $\geq 4$ ) have been found. They are as follow:

1. Statistically significant cycles by duration of $11,19-26, \sim 50-60,80-90$ and 240-250 years in the annual new-generated volcanic eruption number time series by using of two independent methods (the T-R periodogram and wavelet analysis) has been established. They have analogues in the solar and the geomagnetic activity.

2. There are two peaks in the relative and absolute number of strong volcanic eruptions histograms, which corresponds to sunspot minima and sunspot maxima respectively. These peaks are less expressed for the "moderate" strong volcanic eruptions (VEI=4) and better - for the most powerful ones, for which VEI $\geq 5$.

3. Most probably the primary sources of solar (and space weather at all) triggering over volcanic activity are the solar flares and accompanied them phenomena as coronal mass 
ejections (CME's), solar energetic particles (SEP), ground level enhancements (GLE) and geomagnetic storms.

4. It is very possible that solar-volcanic triggering is a serious indirect component of the total "Sun-climate" relationship.

\section{References}

Danjon A., (1921) "Relation Entre l'Eclairement de la Lune Eclipsee et l'Activite Solaire,

L'Astronomie, 35,261

Dergachev V., (1994), Radiouglerodnii Chronometr, Priroda, 1994,No1 (in Russian)

Kaftan V., Komitov B., Lebedev S., (2018), Analysis of sea level, related to cosmo-geophysical processes, based on satellite and terrestrial data, Geodesy and Geodynamics, 9,6, 449

Komitov B., (1986), Possible influence of solar activity on the climate of Bulgaria, Bul.Solnechnie Dannie, 1986, No5, 73 -78 (in Russian)

Komitov B., (1997), The Schove's series. Centural and Supercentural variations of the solar activity. Relationships between adjacent 11-year cycles, Bulg.Geoph.J, 23, 74

Komitov B., Stoychev K., (2011), Stratospheric ozone, solar activity and volcanism, Bulg.Astron. J., 17, 118

Schove D.J., (1983), Sunspot Cycles (Stroudsburg: Hutchinson Ross, Pennsylvania.)

Stotchers R.B., (1989), Volcanic Eruptions and Solar Activity, Journal of Geophys. Res., 94, B12, 371

Strestik J., (2003), Possible correlation between solar and volcanic activity in a long term scale,

Proc. ISCS 2003 Symposium "Solar Variability as an Input to the Earth Environment", Tatranska Lomnica, Slovacia, 23-28 June 2003 (SP-535, September 2003)

Svensmark, H., and E. Friis-Christensen, 1997, Variation of cosmic ray flux and global cloud coverageA missing link in solar-climate relationships, J. Atmos. Sol. Terr. Phys., 59, 1225- 1232

Tchijevskii A., (1976), Zemnoe echo solnechnih bur, Nauka, Moscow, 1976 (in Russian)

Torrence, C. and Compo, G.P., (1998), A Practical Guide to Wavelet Analysis, Bul. Am. Meteor. Soc. 79, 61-78

Vaquero J.M., Gallego M.C., Garcia J.A., (1997), A 250 -year cycle in naked-eye observations of sunspots Geophys. Res. Lett, 29,20

Yu F., (2002), Altitude variations of cosmic ray induced production of aerosols: Implications for global cloudiness and climate, Geophys. Res. Lett., v107, No A7 


\title{
The Beech Tree Ring Widths, Solar-Climatic Relationships and Solar Dynamo Regime Changes
}

\author{
Komitov B. ${ }^{1}$, Kaftan $V .^{2}$ \\ ${ }^{1}$ Institute of Astronomy and NAO - Bulgarian Academy of Sciences \\ ${ }^{2}$ Geophysical Center - Russian Academy of Sciences
}

\begin{abstract}
.
In this study the results from analysis of annual ring widths $D m$ time series of two "very sensitive" to climate and solar-climate relationships long lived beech samples (age of 209 and 245 years respectively) are discussed. The both series are characterized by very good expressed 20-22 year oscillations, related to the solar magnetic Hale cycle. A good coincidence between the changes of $\mathrm{Dm}$ and the forcing or fading of solar magnetic cycle is found. The transition effects at the beginning and ending of the grand Dalton (1793-1833) and Gleissberg minima (1898-1933) are very clear visible in the annual tree ring width data for the one beech samples. Some of these effects are detected also in the second sample. The problem for the possible "lost" sunspot cycle at the end of 18 th century is also discussed.
\end{abstract}

\section{Introduction}

The idea for investigations of solar activity in the past as well as the "Sun-climate" relationships on the base of tree ring widths measurements are suggested by the American astronomer Edward Douglass (1867-1962), the "father" of dendrochronology. He has provided the pioneer studies in this field on the base of long lived tree ring samples manly from the southwest part of USA [Frittz, 1975]. Few decades later the British scientist Derek Schove partly uses dendrochronological data for reconstruction of solar activity in the past (the so called "Schove's series") [Schove, 1955, 1983]. Recently, in $1970^{\text {th }}$ extended study of solar-climatic relationships based on dendrochronological data from the west part of USA has been made. A relative new dendrochrinological studies, which are oriented to "Sun-climate" problematic has been published by Rasspopov et.al [2008].

However, in many cases there are significant uncertainties relating the tree ring widths data and their analysis. They are due to biological features of the wood samples growth. Participating in photosynthesis, the trees consume water and nutrient resources from the surrounding soil, along with a number of growing representatives of the flora - trees, shrubs, and grasses. The latter are competitors in the use of these resources. Therefore, the growth of a tree at its various stages depends, to a certain extent, on the growth rate of the surrounding plants. In this case, additional external factors, such as industrial or sanitary deforestation, windfall, storm erosion of the upper soil layer, the laying of forest roads near the test sample, etc. can play a role. Landscape features, such as the slope of the terrain and the petrochemical composition and physical properties of the soil, also affect the degree of uncertainty in the estimates of the influence of solar activity and climate on tree growth. Trees growing on negative landforms, e.g., in river floodplains, receive abundant water supply from the moisture-saturated soil. Their growth is too inert with respect to atmospheric climatic changes. The effect of precipitation in short-time scales for these cases may not be noticeable

Otherwise, when the tree grows on steep slopes in soils with a small accumulation of water, a more intense influence of solar and climatic factors on the growth of the tree can be expected. In the best such cases on the base of corresponding data it could found and analyzed some fine effects in solar-climatic relationships evolution and connected with the last one solar dynamo regime changes.

The tree rings widths time series investigation of a long lived (age $209 \pm 1$ years) beech sample in aspect of "Sun-climate" and solar dynamo regime changes during the interval $18^{\text {th }}$ - 
$21^{\text {st }}$ centuries is the subject of this paper. Another long lived beech sample (age $245 \pm 5$ years) time series has been also used for some comparisons.

\section{Data and methods}

The both beech samples (Fagus Silvatica) has been obtained in Central Stara Planina (Balkan Mountain Range). The first one, labeled as Gurkovo-01 was logged in 1983 from a site on the south slope of Stara Planina (in Region of Stara Zagora) and $~ 800 \mathrm{~m}$ above sea level. It is $209 \pm 1$ years old. The second sample Rositsa-01 was logged in 2011 in $\sim 50 \mathrm{~km} \mathrm{NW}$ from Gurkovo-01 on the north slope of Stara Planina (in Region of Gabrovo) and $1000 \mathrm{~m}$ above sea level. The age of this sample is $245 \pm 5$ years.

These samples are used in a series of earlier our works, concerning the studies of solarclimatic relationships, based on dendrochronological data from Bulgaria. The measurement and preliminary data proceedings has been described in our previous works (see [Komitov et al., 2013] and cites therein).

Stara Planina is a significant climatic boundary. On the north side of mountain range where the Rositsa-01 site is, the main air transit comes predominantly directly from North Atlantic. Over South Bulgaria, the air transition from Mediterranean Sea (and connected with it Mediterranean cyclones) play also important role. During the $20^{\text {th }}$ century a statistically significant 20-22-year cycle in variability of precipitations and temperatures instrumental series for the warm part of year (April-October) in $~ 70-75 \%$ of South Bulgaria territory has been detected [Komitov, 1986]. In contrary, in North Bulgaria the 20-22-year climatic cycle the last circumstance only for separate few meteorological stations is valid. The terrain of Rositsa-01 site is featured by significantly higher underground water resources as the Gurkovo-01 site. Taken also into account the above mentioned, related to climatic differences between North and South Bulgaria it could in advance to expect that Rositsa-01 tree rings width series is less sensitive to short - time oscillations like 20-22-year solar-climatic cycle as Gurkovo-01. The validity of this suggestion will be demonstrate below.

As is known the 10-11-year and 20-22 year solar cycles are basic phenomena for the solar dynamo theory [Choudhuri, 2007]. However, a statistical significant 11-year cycle signal in the beech samples time series not exist. This is related to the fact that the beech vegetation season is between April and October, where the 20-22-year, but not 11-year solar-climatic cycle is significant [Komitov, 1986]. That's why our study is focused mainly on the 20-22-year cycle amplitude and statistical significance variations in the both tree rings time series.

The time series analysis is based on T-R periodogram algorithm, which is described in few our previous papers (see [Komitov, 1997] and cites therein).

\section{Results and analysis}

The smoothed by 5 years tree rings width time series of both samples is shown on Fig.1.

One of the most impressive features in Gurkovo-01-time series (left panel) are the clear visible solar modulated quasi-cyclic oscillations by duration of $\sim 20$ years during the larger part of investigated period - since $1830 \mathrm{AD}$. The local minima of quasi-20-year oscillations of $\mathrm{Dm}$ correspond to minima of even numbered in the Zurich series sunspot cycles, while the Dm maxima occur predominantly near to odd Zurich sunspot maxima. This fact is in agreement with our earlier results, based on instrumental data for 20th century about tendency for drier and warmer "summer" half-year seasons (May-October) during the even numbered SchwabeWolf's sunspot minima and more wet and colder ones near to even numbered sunspot cycles maxima. The statistically significant negative coefficient of linear correlation $r=-0.69$ between "summer" half-year precipitation in Stara Zagora meteorological station (in Central South Bulgaria) and Dm of Gurkovo-01 beech sample tree widths series during the epoch (1926-1982 AD) supported this statement. On the other side the statistically significance of correlation 
coefficient between the mean "summer" half-year temperatures are even higher $r=-0.71$. Our analysis points out that leading factor for $D m$ changes is the temperature, while the relationship between precipitations and $D m$ is just effect of a strong correlation between the precipitations and temperature.

The second important feature in the left panel on Fig.1 is the serious drop of 20-22-year cycle amplitude during the epoch 1790-1830 AD, which corresponds to the solar grand Dalton minimum.

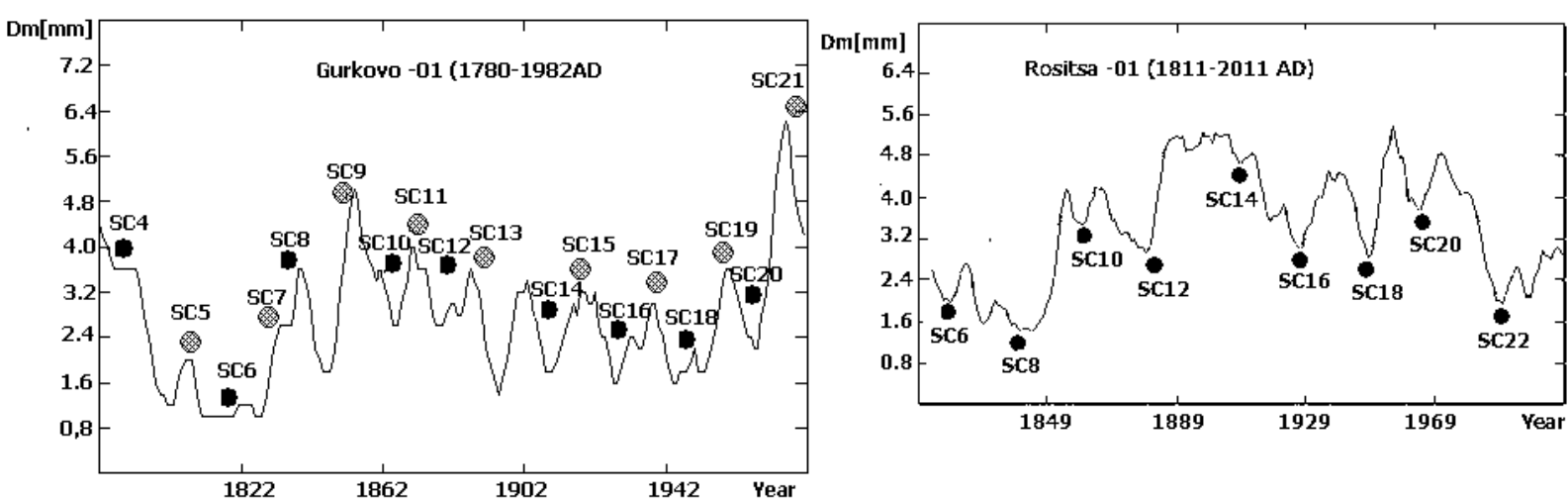

Fig.1. The smoothed 5-year beech annual ring widths series (left: Gurkovo-01; right Rositsa-01). The odd numbered Zurich sunspot cycles maxima are marked by gray spots, the even numbered ones -by black spots.

On the right panel of Fig. 1 the smoothed by 5 years $D m$ time series of Rositsa-01 cycles is shown. There are weak 20-22-year oscillations. They are strongly dominated by quasi-periodic trend by duration of $T=207$ years (see Fig.2, right panel). The both minima of this bi-centurial "hyper-cycle" (also by solar origin [Rasspopov et al., 2008]) are during the Dalton minimum and the present epoch of low solar activity. In contrary, the bi-centurial oscillation in Gurkovo01 series is not visible. May be somewhat it is caused by the fact that this series is ended in 1982 $\mathrm{AD}$, when the new long term solar minimum was not starts yet.

This comparison was useful by the fact that it demonstrates the significance of a large number of conditions, which are strongly necessary to taken into account for more precise analysis of "Sun-climate" relationships and interesting events in solar dynamo regime changes in the past. Gurkovo-01 is the essentially better for studying of the much shorter time scale effects due to the better manifestation of solar magnetic 22-year cycle effects

It has been established in our present study that the statistically significant quasi periodic oscillations in Gurkovo-01 time series by duration of 17, 21, 24, 46, 64 and 108-year are well detectable. All they have analogs in solar and geomagnetic activity, except the 46-year one, which origin is by our opinion unclear on this stage. The 64-year and 108-year are the most featured from all oscillations. Their peak correlation coefficients $R$ are 0.515 and 0.649 respectively. There are also few weaker expressed cycles by duration of 12, 15, 27, 31 and 37 years. The 31-year cycle is equal of $\sim 3$ Schwabe-Wolf's sunspot cycles and could be also considered as resonance related to the 64 -year cycle.

The Rositsa-01 T-R spectra contains statistically significant oscillations by durations of 38 , 54, 84 and 207 years. The first one has analog in geomagnetic activity, while the other three both in sunspots and sunspot activity. As it has been pointed out above the quasi decadal oscillations ( 20-year) in Rositsa-01 are very weak and it is well shown also on the T-R correlogram. That's why the Rositsa-01 time series variations are a good indicator for the longterm solar dynamo changes and long solar-climate tendencies, but essentially less sensitive in short time scales.

A more detailed analysis for the 20-22-year oscillation changes in Gurkovo-01 series has been made by calculating of the integral power index S22 (see [Komitov, 1997] and cites 
therein). For the aims of present study, the possible interval of solar magnetic (Hale) cycle and corresponding to him climate and tree ring widths are choice to be $18-24$ years. The time series of S22 is shown on Fig.2 (left panel), while the corresponding T-R spectra is shown on the right panel. The sharp fading of during the Dalton minimum was follows by epoch of significant higher mean level in the middle of 19th century. Approximately since 1860 AD a sharp downward tendency begins, which reach his minimum at 1910-1912 AD. S22 is going up after that up to 1927-1928 AD. There is a shallow minimum near to 1940 AD. The epoch since 1940 and up to 1980 (Zurich solar cycles No 17-21) is featured by a general increasing of 20-22-year cycle amplitude. As it could to see in T-R correlogram (Fig.2., right) the 20-22-year cycle amplitude is modulated mainly by 64-year cycle and on second place by 107-year one. The third important cycle is by duration of $\sim 32$ years and most probable it is in resonance relationship with the 64-year one.

Quasi-60-year oscillations are detected in many phenomena in space and Earth climate. A 62-year cycle has been obtained in middle latitude aurora (MLA) historical time series and "cosmogenic" isotope ${ }^{10} \mathrm{Be}$ abundances in Greenland continental ice core "Dye-3" [Komitov, 2009]. These facts lead to a very high probability to a hypothesis that the $\sim 60$-year cycle have a solar origin. The problem is very intriguing if taken into account that there is no significant manifestation of 5 or 6 sunspot cycles in sunspot indexes or they are weak if exist.

It needs especially note that a cyclic tendency of $\sim 60-65$-years follows from the study of Georgieva [2011] concerning the possible origin of the Schwabe-Wolf's cycle double peaked structure and the Gnevishev's gap between them. Based on sunspot activity data for 20th century she suggests that the both peaks of the $\sim 11$-year sunspot cycle are caused by two types of plasma transport in Sun's convective zone - advective and diffusive. Every one of these two gives its separate part in sunspot activity, which maxima are shifted meanly on $1.5-2$ years each from other. They correspond to the both main peaks of sunspot activity in Schwabe Wolf's cycle. By our opinion there is an oscillation variable regime of the both types of plasma transport by length of $\sim 6$ Schwabe-Wolf's cycles, i.e. $\sim 60-70$ years. The order reverse of the both sunspot peaks ("advecive - diffusive" to "diffusive - advective" and on the contrary) occurs meanly on 3 sunspot cycles, i.e. approximately $30-35$ years. Thus the $\sim 60-65$-year cycle could exist in solar dynamo without any significant manifestation in sunspot activity. On the other hand, these phenomena seems to play role in manifestations of solar flares, coronal mass ejections (CMS) as well as the solar wind parameters, geomagnetic storms and aurora activity.

We find that the detected the Gurkovo-01 sample series main local extrema of S22 are in good coincidence with the double types plasma transport scheme since the beginning of 20th century thereafter enough certain corresponding data from the sunspot observations are extracted. Due to this 60-year cycle there is an interleaving of 20-22-year cycles by high, moderate and weak cycle magnitude in the tree ring widths data, which is very well expressed in Gurkovo-01 beech sample. A 60-year cycle is detected in many Earth climate parameters, for example in the Northern hemisphere temperatures [Thompson, 1997]. Quasi-60-year variations has been detected in North Atlantic Oscillation behavior [Mazzarella and Scafetta, 2012]. 

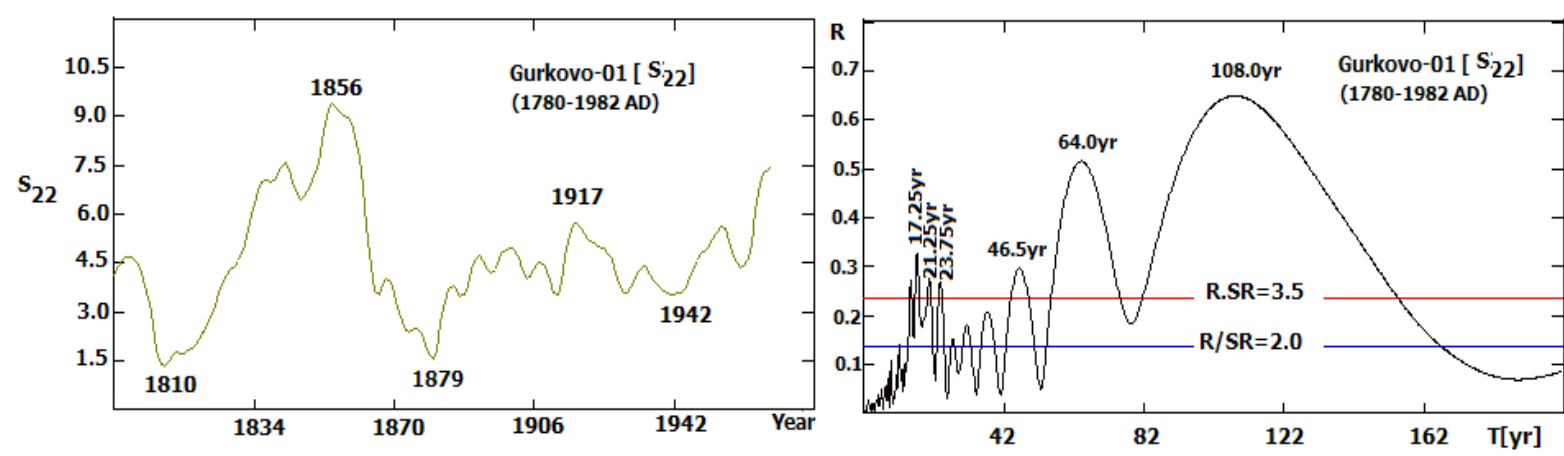

Fig. 2 Left: The amplitude index S22 of Gurkovo-01 beech sample Dm-series. The moving window width is equal to 30 years; right: the corresponding T-R correlogram.

By our opinion the relative high sensitivity of Gurkovo-01 to the short scale solar activity oscillations like 20-22-year Hale cycle opens a possibility for more detailed study of the solar activity long term changes effects such as the transitions from high solar activity epochs to grand solar minima. In the Gurkovo-01 time series such phenomena could to observe in the end of 18th century - the transition epoch between sunspot cycle No 4 (SC4) and the weak next SC5. According almost all researchers it marks the beginning of grand Dalton minimum (17981825 AD). An exclusion of this "consensual" scenario is the suggestion for an additional weak and short "lost" sunspot cycle after SC4 [Usoskin et al, 2001]. This weak sunspot cycle SC4 should be started in 1793 AD and ends approximately in 1799/1800 AD. This hypothesis was very popular in 2000th, but finally it was not appropriated. However, all researchers agree that there was occurs an unusual sunspot activity behavior in 1790th, which indicates for a serious corruption of Schwabe Wolf's cycle during this time.

As it shown on Fig.1 the maximum of the even SC4 sunspot cycle coincided with an inflection on the graphics of $\mathrm{Dm}$, while the smoothed moments of the remained even sunspot maxima correspond to $\mathrm{Dm}$ minima, i.e. local temperature maxima and precipitation minima. The "inflection" near to SC4 is very short and it was followed not by increasing but by an unexpected and even more strong and continuous decreasing.

The smoothed maximum of the next odd numbered SC5 corresponds to weak Dm maximum. Thus a serious destroying of 22-year solar cycle influence over tree ring widths growth is indicated during the last decade of 18th century. The "critical" moment is 1793 AD where by our opinion occurs the real start of Dalton minimum. The typical downward phase of sunspots in SC4 was break near to this calendar year, as it seems suddenly by some new phenomena, which forced the sunspot activity decreasing. Such phenomena potentially could be the Sun's tachocline region sinking. As it is shown on the same panel SC5 maximum corresponds to a weak, but clear visible $\mathrm{Dm}$ maximum. This fact indicates that there is no change in "Dm-22-year solar cycle phase" relationship between SC4 and SC5 maxima. Consequently, there is no indication for some additional Schwabe-Wolf's cycle at the beginning of Dalton minimum.

There is an indication about significant "surplus" $\mathrm{Dm}$ minimum near the end of 19th century ( 1898/1899 AD). It is not in coincidence with even numbered sunspot cycle maximum, but with the minimum of the even SC14. This calendar moment is also estimated as a start of the grand Gleissberg sunspot minimum (1898-1923 AD).

\section{Conclusions}

Evidences about successful using of some tree ring data series for "Sun-climate" relationships and solar dynamo regime investigations on the base of presented their results and 
their analysis are given in this study. Such data series, obtained from long lived (age 200 year) could be a good indicator for reconstruction both of terrestrial and space climate in preinstrumental era.

\section{References}

Frittz, H.G. (1975). Tree Rings and Climate, Acadeic Press, London-New York-San Francisco.

Choudhuri, A.R. (2007). An Elementary Introduction to Solar Dynamo Theory. (Preprint), July 2007, doi: $10.1063 / 1.2756783$

Georgieva K. (2011). Why the sunspot cycle is double peaked? ISRN Astronomy and Astrophysics, V2011, Article ID 437818,2011, doi 10.5402/2011/437838

Komitov, B. (1986). Possible Influence Of Solar Activity on the Climate in Bulgaria, Bul. Solnechnye Dannie, 1986, No3, 73-78, 1986.

Komitov B. (1997). The Schove's series. Centural and Supercentural variations of the solar activity. Relationships between adjacent 11-year cycles, Bulg.Geoph.J, 23, 74, 1997.

Komitov B. (2009). The "Sun-climate" relationship: II. The "cosmogenic" berryylium and the middle latitude aurora. Bulg. Astron. J., 12, 75-90, 2009.

Komitov B., Duchlev P., Bjandov G., Kirilova D. (2013). Trees Annual Rings and "Sun-Climate" Connection, Bulg.Astron. J., 19, 72, 2013.

Mazzarella A., Scafetta N. (2012). Evidences for a quasi-60-year North Atlantic Oscillation since 1700 and its meaning for global climate change, Theoretical and Applied Climatology, 107, iss.3-4, 599-609, 2012, DOI: 10.1007/s00704-011-0499-4

Rasspopov, O.M., Dergachev, V.A., Esper, J., Kozyreva, O., Frank, D., Ogurtsov, M., Kplstrom, T., Shao, X. (2008). The influence of the de Vries ( 200 year) solar cycle on climate variations: Results from Central Asia Mountains and their global link, Paleogeography, Paleoclimatology, Paleoecology, 259, 6-16, 2008, https://doi.org/10.1016/j.palaeo.2006.12.017

Schove, D. J. (1955). The Sunspot Cycle 649 BC to AD 2000, J. Geophys. Res., 60, 127-146, 1955, https://doi.org/10.1029/JZ060i002p00127

Schove D.J. (1983). Sunspot Cycles (Stroudsburg: Hutchinson Ross, Pennsylvania.)

Thompson D. (1997). Dependence of global temperatures on atmospheric CO2 and solar irradiance, Proc. Nat. Acad. Sci. USA, 94, 8370-8377, 1983

Usoskin I., Mursula K., Kovaltsov G. (2001). Was one sunspot cycle lost in the late XVIII century? Astron. Astrophys., 370, L31-L34, 2001, DOI: 10.1051/0004-6361:20010319 


\title{
Observations Supporting Hypothesis for Global Electrical Circuit as Mediator between Solar Events and Weather
}

\author{
Tonev P.T. \\ Space Research and Technology Institute BAS, Sofia, Bulgaria \\ E-mail: ptonev@bas.bg
}

\begin{abstract}
.
Some of the few available experimental studies are discussed which demonstrate peculiarities of the local response of the global atmospheric electrical circuit (GEC) to major solar proton events (SPE), concerning conductivity, the fair-weather electric current $J_{z}$ from ionosphere to ground, and the related field $E_{z}$. For more representative picture, these experiments include auroral and lower latitudes, as well as different altitudes: the ground level, stratosphere and mesosphere. The experimental data show typical peculiarities: although these are not fully understood yet, they can give a key to the global response of GEC to SPE. It is concluded that a relevant 3D dc model of GEC is necessary to determine factors and physical conditions which would lead to the locally observed characteristics.
\end{abstract}

\section{Introduction}

The aim of this work is to demonstrate the specific peculiar response of the global atmospheric electrical circuit (GEC) during major solar proton events (SPEs) seen from the available experimental studies performed in different atmospheric locations. These comprise measurements of the main electrical characteristics (conductivity $\sigma$, electrical field $\mathbf{E}$ and current density $\mathbf{J}$ ) within several groups: a) at high and middle latitudes - balloon experiments at altitudes between $26 \mathrm{~km}$ and $31 \mathrm{~km}$, as well as rocket measurements in stratosphere and mesosphere; b) ground-based measurements at high and low latitude. All these experimental studies demonstrate peculiar and large variations which cannot be predicted theoretically [Farrell and Desch, 2002]. Some factors are formulated in this work which can be responsible for the demonstrated peculiar behavior, together with their further analysis. The main future goal is to clarify the global-scale behavior of GEC during SPEs. Recent studies demonstrate that SEP can affect weather [Veretenenko, 2017], with GEC as hypothetical mediator between solar drivers and weather formation. According to the hypothesis [Tinsley, 2000] weather formation is affected by variations of the electric current $J_{z}$ of few $\mathrm{pA} / \mathrm{m}^{2}$ which flows in GEC from the ionosphere to the surface in fair-weather regions where $J_{z}$ influences creation of cloud and the atmospheric circulation.

\section{Peculiarities demonstrated from experimental results in stratosphere}

\section{a) Measurements in Antarctic stratosphere during SPE'69 (GLE) on 20 January 2005}

Fig.1 demonstrates data from GOES-10 for the solar proton flux during SPE'69 on 20.01.2005 (the upper-left panel), together with the simultaneously obtained balloon-borne data for the conductivity $\sigma$ (the lower-left panel), the vertical electric field $E z$ (the upper-right panel), and the related current density $J z$ (the lower-right panel) in Antarctic stratosphere at coordinates between $\left(70.9^{\circ} \mathrm{S}, 10.9^{\circ} \mathrm{W}\right)$ and $\left(71.4^{\circ} \mathrm{S}, 21.5^{\circ} \mathrm{W}\right)$, and at altitude $30.9-33.2 \mathrm{~km}$ [Kokorowsky et al., 2006; 2012]. Some of the peculiarities of the observed characteristics (not satisfactorily explained yet) are discussed by us here. Before the SPE onset at 06:51 UT $\sigma, E z$, and $J z$ have typical values: $\sigma$ is few $\times 10^{-10} \mathrm{~S} / \mathrm{m}$; the electric current $J z$ is downward, with mean value $\sim 2$ $\mathrm{pA} / \mathrm{m}^{2}$, however $E z$ and $J z$ are unusually dispersed. After SPE onset, till 13:48 UT: $i$ ) the conductivity $\sigma$ increases due to ionization by protons with energies $\sim 100 \mathrm{MeV}$ - initially $\sim 20$ times or more; $i$ ) $E z$ initially suddenly drops almost to zero, then $E z$ increases to its usual values; iii) $\mathrm{J} z$ shows large variations to values about 5 times larger than usually. In the period 
13:48-5:58 UT conductivity exceeds 5-10 times its usual values, and $E z$, as well as $J z$ remain close to zero. After 15:58 UT $E z$ and $J z$ suddenly change their direction from downward to upward for at least 5 hours, yet $J z$ exceeds more than twice its typical value. This occurred together with the sudden increase of protons with energies $<5 \mathrm{MeV}$ which do not reach stratosphere. Also, a geomagnetic storm (12:00-18:00 UT; Kp=4o, 5-), and a strong sub-storm from 15:00 till 18:00 UT (with AE 1200 nT) occurred. The current $J z$ remained reversed to upward for hours, yet exceeds more than twice its typical value (these last features are hard to explain). Although SPE'69 causes dramatic modification of conductivity $\sigma$ in the middle atmosphere at high latitudes due to strong ionization, as demonstrated in Fig.2 [Usoskin et al., 2014], the model results by the well-known oversimplified 1D model presentation of GEC by an equivalent circuit predicts that changes of $J z$ are $<\sim 5 \%$; thus, this model does not predict the large and long-time variations in Fig.1.
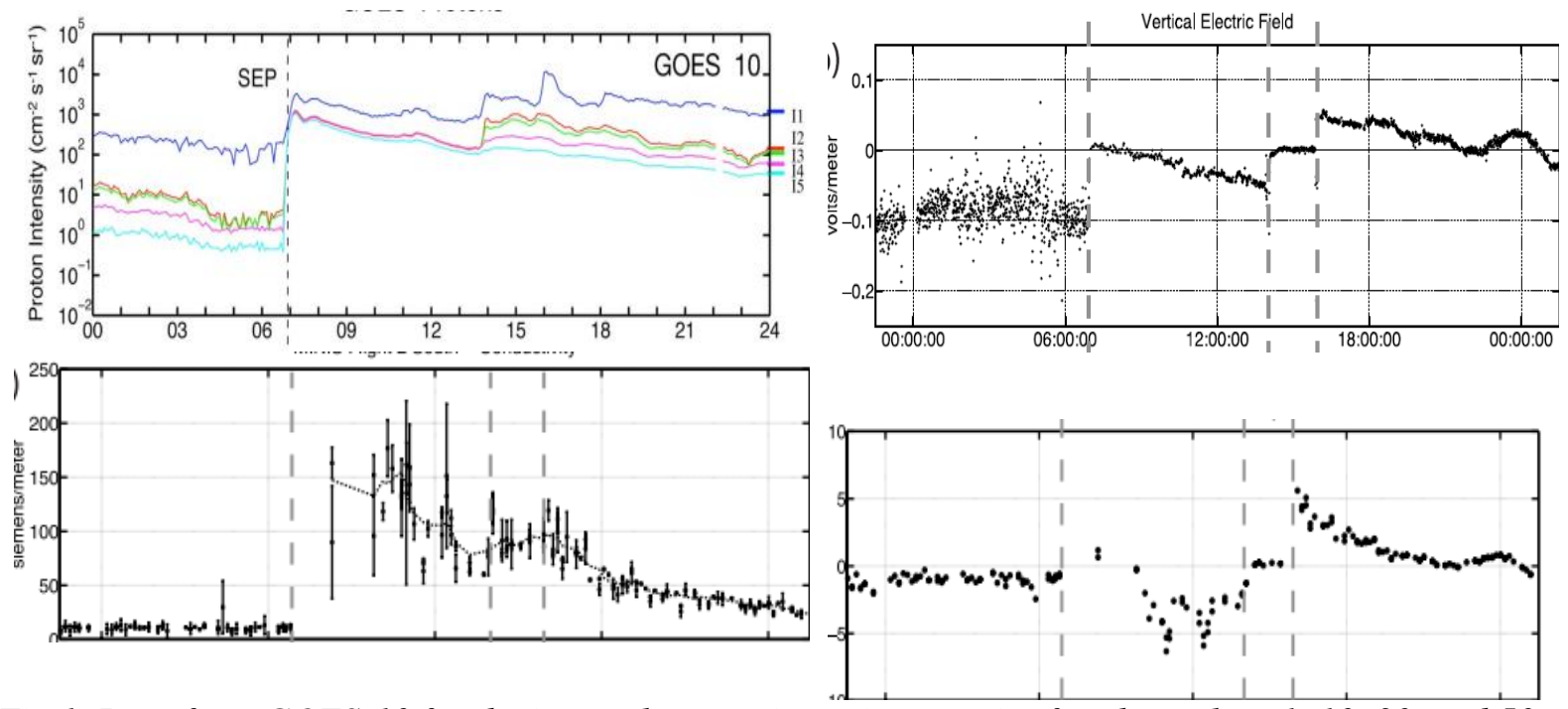

Fig.1. Data from GOES-10 for the integral proton intensity energies for channels $>1,10,30$, and 50 $\mathrm{MeV}$ (the upper-left panel); simultaneous measurements of conductivity $\square$ (the lower-left panel); vertical electric field $E z$ (the upper-right panel); and the vertical current density $\mathrm{J} z$ (the lower-right panel) computed from $\square$ and Ez [Kokorowski et al., 2006; 2012].

Similar model estimations are obtained also by [Farell and Desh, 2002], in contradiction with experimental data for $E_{z}$ and $J_{z}$.

\section{b) Measurements in stratosphere at middle latitudes during SPE on 16 February 1984}

During SPE (with GLE) on 16 Feb.1984 the electric characteristics $\sigma, E z$, and $J z$ were obtained on a balloon payload EMA-8 with coordinates $\left(44.6^{\circ} \mathrm{S}, 142.7^{\circ} \mathrm{E}\right), \Lambda=-56.3^{\circ}$ [Hozlworth et al., 1987]. Data obtained are presented in Fig.2. This SPE occurred in short time period ( one hour). Simultaneously was obtained data from identical payload EMA-7 located on lower latitude $\left(\Lambda=-48.8^{\circ}\right)$ which indicated no effect of SPE on $\sigma, E z$, and $J z$. Both $\sigma$ and $E z$ increased initially ( $\sigma$ enhanced more than twice); $J z$ also increased (2.2 times). Peculiarities of perturbations of $\sigma, J z$, and $E z$, though smaller and in shorter time, resemble those demonstrated in Fig.1. These perturbations are also in contradiction with modeling [Farrell and Desch, 2002], and are even more striking than in the former case, since they take place at latitude with much higher cutoff rigidity (> $1 \mathrm{GV})$.

\section{Features of vertical electric fields in mesosphere and stratosphere}

In a series of rocket experiments above Heiss Island $\left(80^{\circ} 37 \mathrm{~N}, 58^{\circ} 03 \mathrm{E}\right)$ and above southern Indian Ocean [Zadorozhny et al., 1998] found unusually large $(\sim 1 \mathrm{~V} / \mathrm{m})$ vertical electric field 
$E z$ in the mesosphere which still remain unexplained. Fig.3 demonstrates profiles of $E z$ in three cases above Indian Ocean. On 21.10.1989 19:31 UT, at $\left(58.5^{\circ} \mathrm{S}, 51.1^{\circ} \mathrm{E}\right), \Lambda=62.7^{\circ} \mathrm{S}$ the electric field $E z$ (presented by thick solid line) reached much larger peak in mesosphere (12.2 $\mathrm{V} / \mathrm{m}$ ) than in all other cases (the second largest peak $E z$ was for 12.10 .1989 shown in Fig.3 by dashed line). The first case on 21.10 coincided with both strong solar proton event and major geomagnetic storm (with $\mathrm{Kp}=8$ ) which cause the enhancement of $E z$.

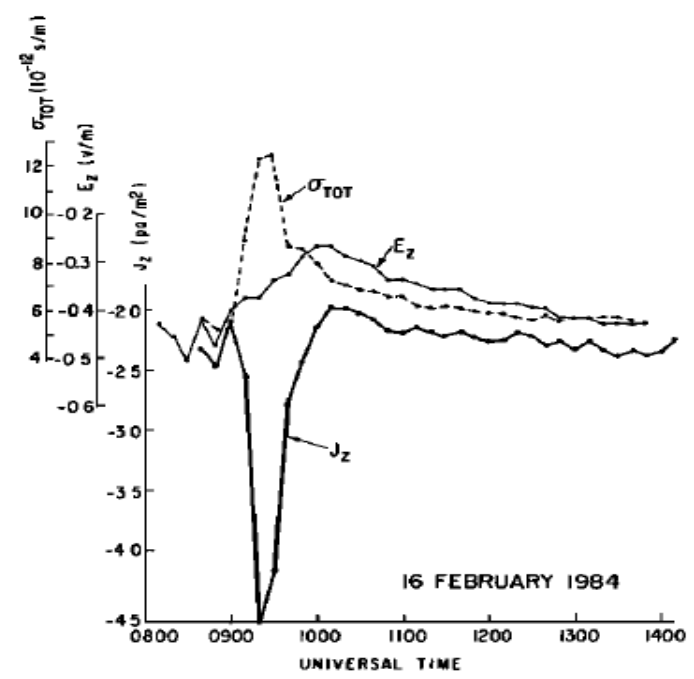

Fig.2. Conductivity $\sigma_{T O T}$, vertical electric field $E_{z}$ and current density $J_{z}=\sigma E_{z}$ during $S P E$ on 16 Feb.1984 from balloon-borne measurements at $26 \mathrm{~km}$ altitude, at (44.6 $\left.{ }^{\circ}, 142.7^{\circ} \mathrm{E}\right), \Lambda=-56.3^{\circ}$.

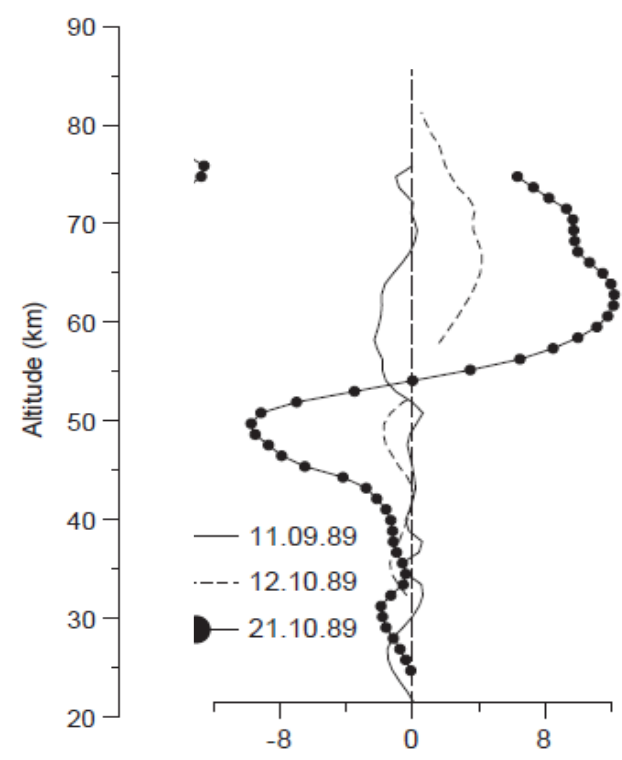

Fig.3. Vertical electric fields Ez measured in Southern Indian Ocean in 1989 on: 11 September 20:15

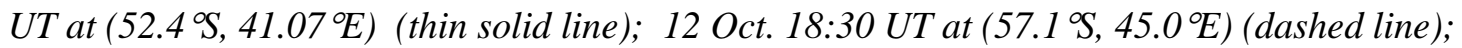
21 Oct., 19:31 UT at (58.5 9 S, $51.1^{\circ} \mathrm{E}$ ) (thick solid line). First two cases are during quite geomagnetic conditions. The last case is during a major geomagnetic storm $(K p=8)$ and GLE.

The following peculiarities are observed: i) Paradoxically large and still unexplained electric fields $E z$ are observed in mesosphere and stratosphere in large number of cases; in Southern hemisphere $E z$ peaks are often much bigger than those in Heiss Island $\left(80.6^{\circ} \mathrm{N}\right)$; ii) usually, direction of the vertical electric field $E z$ changes at $\sim 50 \mathrm{~km}$, but while on $11.09 \mathrm{Ez}$ points to downward above $50 \mathrm{~km}$ and to upward below, in the other two cases it is upward 
above $50 \mathrm{~km}$ and downward below. The positive and the negative $E z$ peaks have comparable values. The source of the large electric fields in mesosphere is under debate. [Zadorozhny et al., 1998], etc. suggested as hypothetical factors effects of charged aerosol particles and decreased conductivity.

\section{Behavior of potential difference $E z$ at surface during SPEs}

\section{a) High latitudes (Kola Peninsula) during two major SPEs in 2001}

Measurements of air-earth current (AEC) $J z$ and the potential difference (PD) $E z$ at ground level at high latitudes have been realized in very few places and for limited periods of time. Fig.4 demonstrates observed variations of $E z$ in Apatity (Kola Peninsula, Russia) at geomagnetic latitude $63.3^{\circ}$ during two major SPE (with GLE) in 2001: SPE-1 on 18 April, and SPE-2 on 4 November [Kasatkina et al., 2009]. In both cases CMEs took place. The following features are valid: 1) Variations of $E z$ strongly enhance after the beginning of SPE; ( $E z$ can enhance up to $\sim 1 \mathrm{kV} / \mathrm{m}$ ); 2) For both SPEs the behavior of $E z$ in the first and the second phase of a SPE is quite different: in the first phase $E z$ varies strongly, yet remains positive; in the second phase $E z$ becomes sometimes negative; its negative peaks can be as large as positive ones.
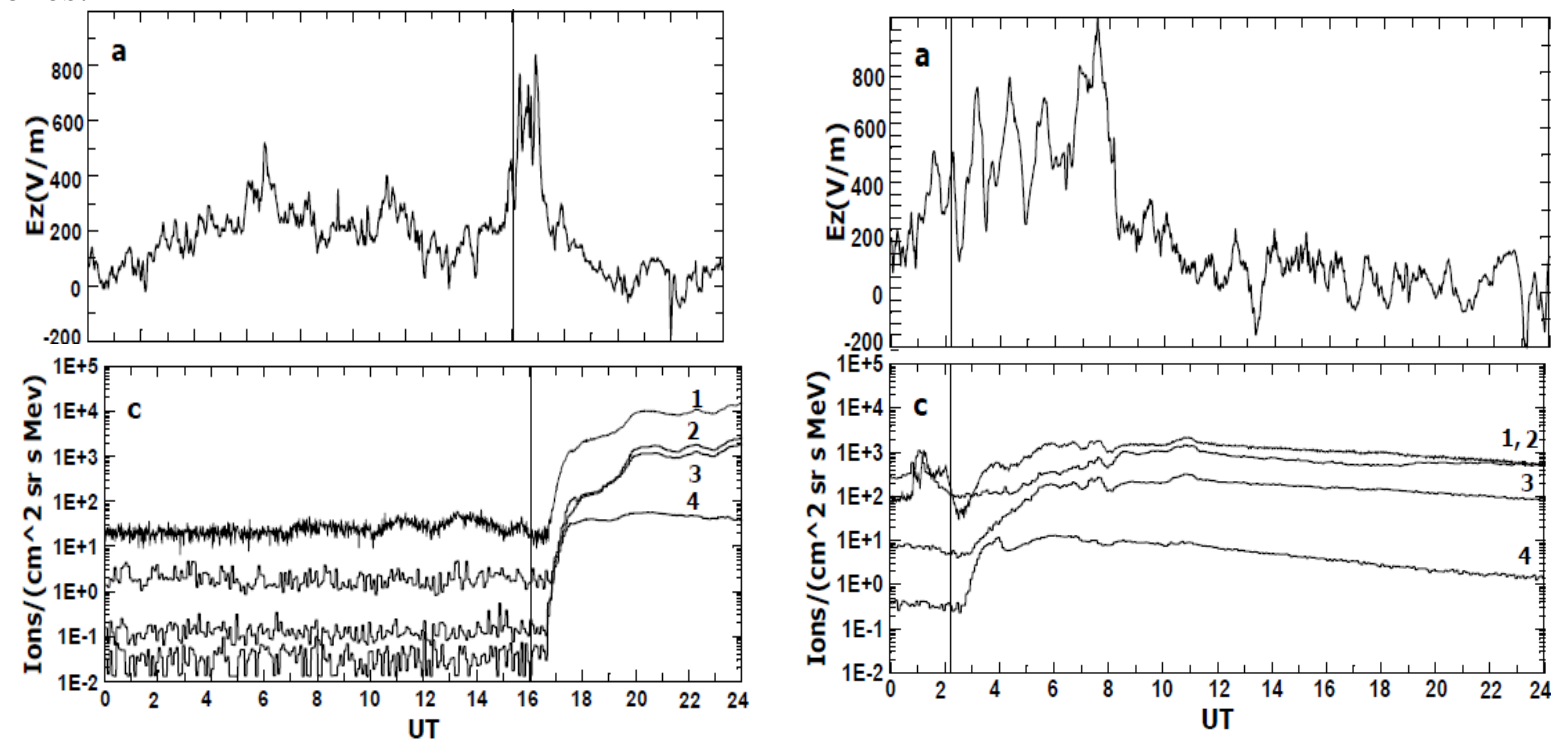

Fig.4. Potential difference Ez at surface in Apatity during SPE-1 on 18.04.2001 (left upper panel) and SPE-2 on 04.11.2001 (right upper panel). The two lower panels show the integral proton intensity energies for channels $>1,10,30$, and $50 \mathrm{MeV}$ (marked by numbers from 1 to 4 for SPE-1 and SPE-2, respectively. The vertical line marks the flare onset.

\section{b) Low latitudes}

Fig.5 demonstrates ground-level variations of the potential difference $E_{z}$, (denoted here as atmospheric electric field, AEF) at low latitudes: in CASLEO, Argentina $\left(31.8^{\circ} \mathrm{S}, 69.3^{\circ} \mathrm{W}\right)$ at $2552 \mathrm{~m}$ altitude during SPE (GLE) on 17.05.2012 [Tacza et al., 2017]. Data for $E_{z}$ in the upper and the lower panels shown by black lines are obtained respectively from measurements at two stations, CAS1 and CAS2. The red lines in both panels show the average electric field $E_{z a}$. Data show significant and typical deviations for a period of $\sim 10$ hours from the SPE onset at 0 UT. During the first phase (for 3.5 - 4h) $E_{z}$ is well smaller than $E_{z a}$; then, for the next 5-6 hours, $E_{z}$ becomes well larger than $E_{z a}$, as indicated by arrows. Similar features take place also for a group of 8 solar proton events: during the first phase of SPE the measured electric field $E_{z}$ is lower than its average value for few hours, and the relation is opposite in the second (somewhat longer) phase of SPE. This pattern of $E_{z}$ during SPE at low latitudes is 'reversed' compared to that high latitudes (although the last is stronger expressed). This indicates that the global-scale reaction of GEC to SPE is of interest. 


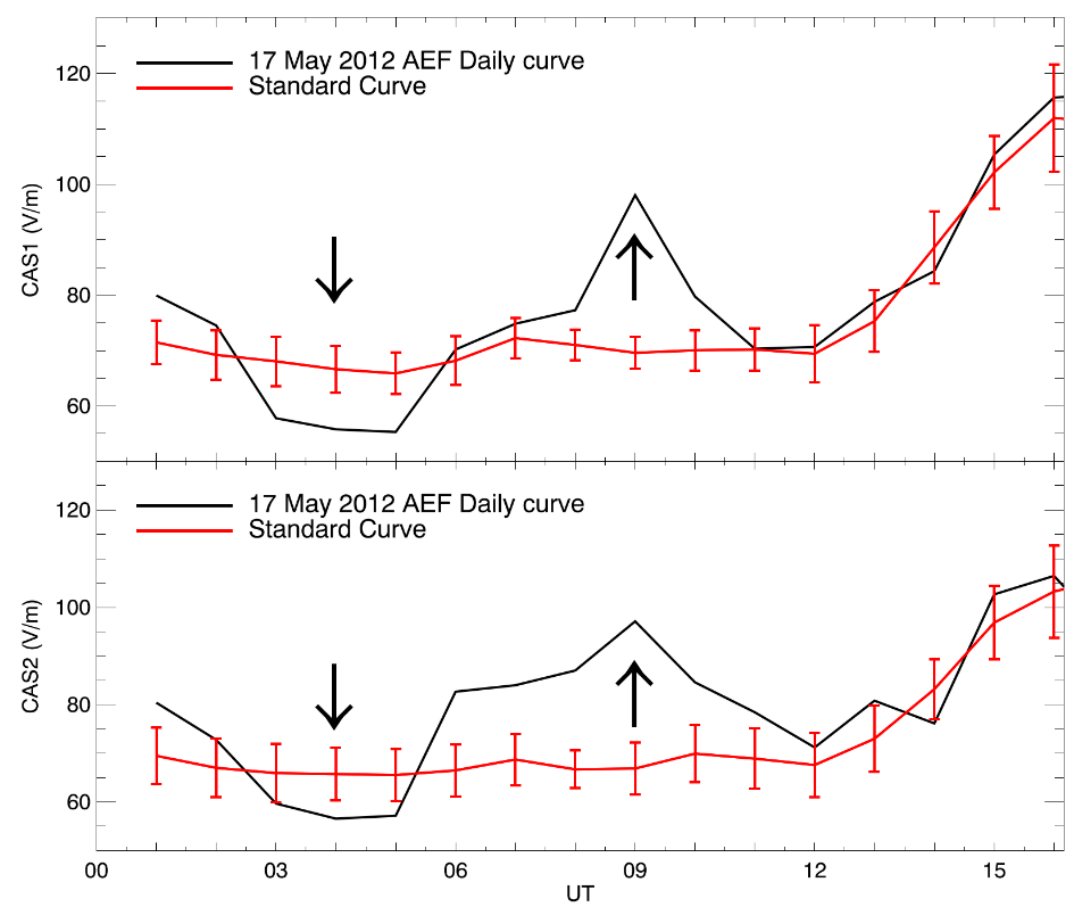

Fig.5. Atmospheric electric field (AEF) measured at CASLEO, Argentina $\left(31.798^{\circ} \mathrm{S}, 69.295^{\circ} \mathrm{W}\right)$ at 2552 m altitude, during GLE on 17 May 2012 [Tacza et al., 2017].

\section{Factors hypothetically possible to enforce effects of SPE on GEC}

All considered here results in Figures 1-5 demonstrate that the response of GEC to a SPE is much stronger and specific than predicted by modeling [Farrell and Desch, 2002]. For a possible explanation, four factors are considered in this work as hypothetically responsible for the specificity of the considered measurements.

Factor 1. Increase of conductivity $\sigma$ in middle atmosphere at high latitudes by enhanced ionization by solar proton flux.

Factor 2. More effective electrical coupling of auroral ionosphere with GEC during SPE due to enhanced conductivity in middle atmosphere at high latitudes. Even penetration of a tiny part $J_{\text {IS }}$ of FAC (say, $\sim 10^{-6}$ ) into the middle atmosphere (due to larger conductivity during SPE) could be significant as superposed current in GEC. The total ionosphere-to-ground current $J z$ would be then $J_{z}=J_{T S}+J_{I S}$ where $J_{\mathrm{TS}}$ is the current from tropospheric sources [Tonev, 2018]. The relation between the geomagnetic activity and the current density $J_{\mathrm{z}}$ in auroral stratosphere has been first supported experimentally by balloon-borne data series [D'Angelo et al., 1982].

Factor 3. A series of studies demonstrate that SPE can produce aerosol particles in the stratosphere [Mironova et al., 2014] and thus can cause decrease of the conductivity.

Factor 4. Penetrating energetic solar protons during SPE could create regions with charged particles, leading to formation of electric fields.

In order to take into account, the possible effects of these factors on the electric characteristics new more adequate models of GEC (2D and 3D, as well as steady-state and quasi-statistic) have to be developed as future tasks.

\section{Conclusions}

1. Peculiar variations of the vertical electric field Ez and related current density Jz are observed during major SPEs at different altitudes and latitudes which are not explained in terms of equivalent electrical circuit. The responsible mechanisms should be searched.

2. Four factors are formulated which can play role in creation of the observed specific and large local variations in GEC during SPE.

3. Development is needed of 2D and/or 3D model of GEC with account to Factors 1-4. 


\section{References}

Farrell, W.M., Desch, M.D. (2002). Solar proton events and the fair weather electric field at ground, Geophys. Res. Lett., 29, no.9, 1323, 10.1029/2001GL013908, 2002.

Hozworth, R.H. Norville, K.W., Williamson, P.R. (1987). Solar flare perturbations in stratospheric current systems, Geophys. Res. Lett., 14, 8, 52-855, 1987.

Kasatkina E.A., Shumilov O.I., Rycroft M.J., Marcz F., Frank-Kamenetsky A. V. (2009). Atmospheric electric field anomalies associated with solar flare/coronal mass ejection events and solar energetic charged particle “Ground Level Events" Atmos. Chem. Phys. Discuss., 9, 21941-21958, 2009.

Kokorovski, M., Sample, J.G., et al. (2006). Rapid fluctuations of stratospheric electric field following a solar energetic particle event, Geophys. Res. Lett., 33, L20105.

Kokorowski, M., Seppälä, A., et al. (2012). Atmosphere-ionosphere conductivity enhancements during a hard solar energetic particle event, J. Geophys. Res., 117, A05319.

Tacza J.C., Raulin, J.-P, and De Juli. M.C. (2017). Solar effects on the Global Atmospheric Electric Circuit, 32nd URSI GASS, Montreal, 19-26 August 2017

Tinsley B.A. (2000). Influence of solar wind on the global electric circuit, and inferred effects on cloud microphysics, temperature and dynamics in the troposphere. Space Sci. Rev., 94, 231-258.

Tonev, P. (2018). Electrical coupling of auroral ionosphere with lower atmospheric regions during SEP, Proc. 10th Workshop "Solar Influences on the Magnetosphere, Ionosphere and Atmosphere", Bulgaria, June 4-8, 2018.

Usoskin, I.G., Kovaltsov, G.A. (2011). Ionization effect of solar particle GLE events in low and middle atmosphere. Atmos. Chem. Phys., 11, 1979-1988.

Veretenenko, S.V. (2017) Особенности прстранственно-временной структуры эффектов солнечной активности и вариаций космических лучей в циркуляции нижней атмосферы, DSc.Thesis, Sanct Pertersburg University, 2017, 327 p.

Zadorozhny, A.M., A.A. Tyutin (1998). Effects of geomagnetic activity on the mesospheric electric fields, Ann. Geophysicae 16, 1544-1551, 1998. 


\title{
Effects of Solar Proton Events of January 2005 on Intensity of the Stratospheric Polar Vortex in the Northern Hemisphere
}

\author{
Veretenenko S.V. \\ Ioffe Institute RAS, St. Petersburg, Russia \\ E-mail: s.veretenenko@mail.ioffe.ru
}

\begin{abstract}
.
Influence of Solar Proton Events (SPEs) of January 2005 on the state of the stratospheric polar vortex in the Northern hemisphere was investigated, the NCEP/NCAR reanalysis data being used. It was revealed that the events under study were accompanied by a noticeable increase of western wind velocities in the latitudinal belt $50-80^{\circ} \mathrm{N}$ (intensification of the polar vortex) at all the stratospheric levels. The obtained results allow suggesting that ionization changes in the stratosphere associated with powerful SPEs may influence the state of the polar vortex on the day-to-day timescale. A possible reason of the vortex intensification may be changes of the thermal-radiative balance of the polar stratosphere resulting from changes of its chemical composition caused by solar proton intrusion.
\end{abstract}

\section{Introduction}

The polar vortex is a large-scale cyclonic circulation forming in the polar atmosphere, with highest values of wind velocity being observed in the upper stratosphere during the cold half a year. The state of the polar vortex influences significantly the development of dynamic processes in the troposphere [Baldwin and Dunkerton, 2001]. The vortex is an important link between solar activity and circulation of the lower atmosphere at extratropical latitudes, since the vortex location, both latitudinal and altitudinal, is favorable for different physical mechanisms of solar-atmospheric relations. First of all, the vortex is formed in the area of low geomagnetic cutoff rigidities allowing energetic charged particles, such as galactic cosmic rays (GCRs) in a broad energy range and solar cosmic rays, to penetrate there producing ionization changes. Precipitations of auroral electrons, as well as high-energy electrons from the radiation belts also take place in this area. Ionization changes associated with variations of these particle fluxes may influence chemical composition and temperature of the polar atmosphere (e.g., [Krivolutsky and Repnev, 2009]). On the other hand, the vortex formation area also includes the area of noticeable changes of ionospheric potential caused by interplanetary magnetic field variations; this provides favorable conditions for electric mechanisms of solar activity influence on atmospheric circulation, which involve variations of vertical electric currents and cloud formation [Tinsley, 2008, 2012]. It was shown [Veretenenko and Ogurtsov, 2019] that variations of the vortex strength play an important part in the formation of GCR effects on the development of extratropical cyclogenesis. So, investigating effects of different helio/geophysical factors on the state of the stratospheric polar vortex is of great importance to clarify the physical mechanisms of solar activity influence on the lower atmosphere circulation, weather and climate. In this connection, the aim of this work is to consider effects of powerful Solar Proton Events of January 2005 on the polar vortex intensity in the stratosphere of the Northern hemisphere.

\section{Solar Proton Events of January 2005}

In January 2005, at the deep descending phase of the $23^{\text {rd }}$ solar cycle a series of strong Solar Proton Events took place (Fig.1a). The events starting on 15, 16 and 17 January were accompanied by the enhancements of particle fluxes in the energy range 165-500 MeV, which can reach stratospheric heights $(\sim 15-30 \mathrm{~km})$ losing their energy to ionization. The most powerful event occurred 20 January. It was accompanied by the enhancement of solar proton flux with energies $>500 \mathrm{MeV}$, which participate in nuclear interactions resulting in the increase 
of the neutron monitor (NM) count rate, so called Ground Level Enhancement (Fig.1b). The SPEs under study were associated with the increase of solar flare activity during the period 1323 January (Fig.1c) and produced significant changes of ionization rate in the stratosphere. Daily mean ionization rates at geomagnetic latitudes $60-90^{\circ}$ are presented in Fig. $1 \mathrm{~d}$ according to the SOLARIS-HEPPA data (https://solarisheppa.geomar.de/).
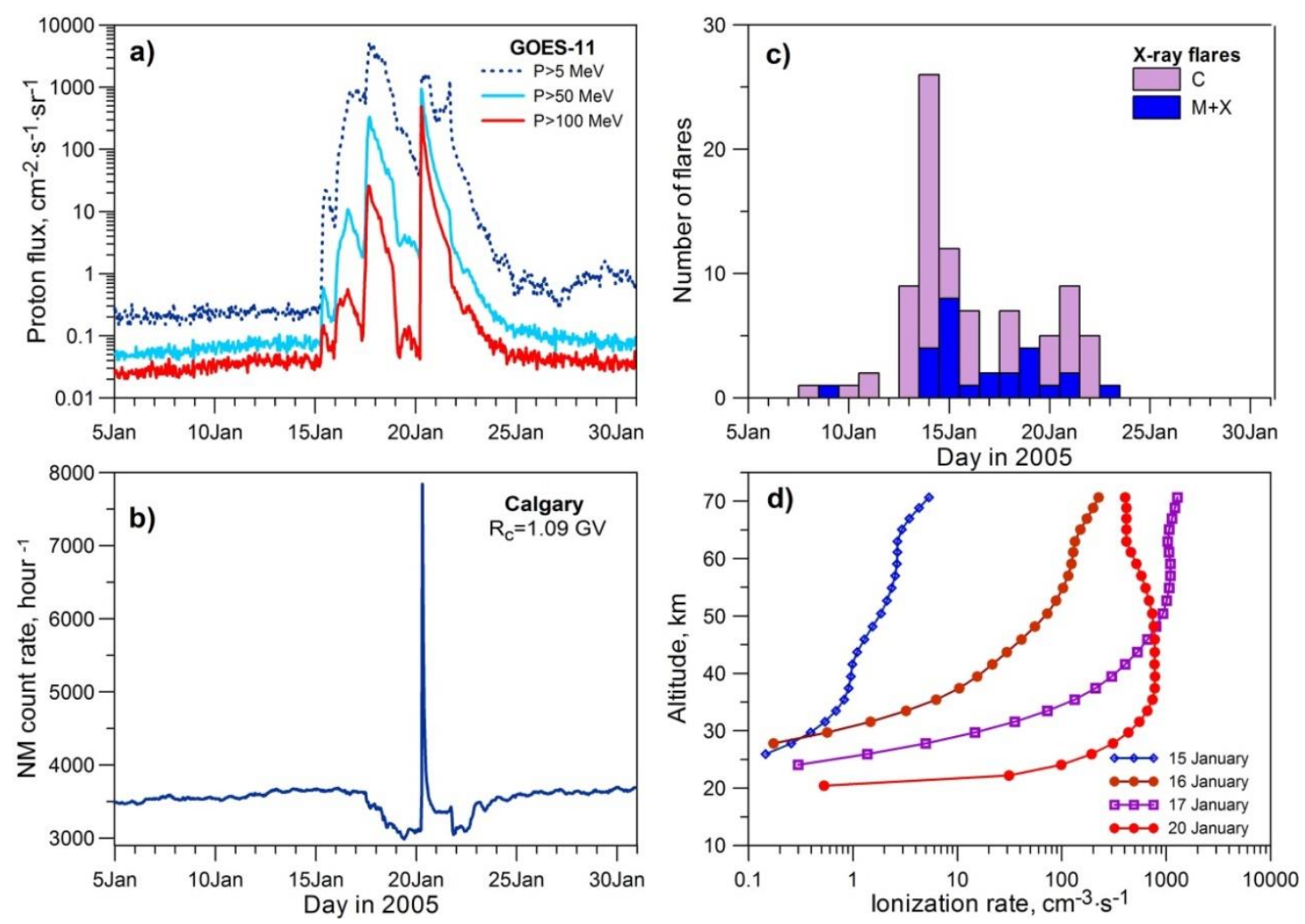

Fig.1. a) Integral solar proton fluxes at different threshold energies ( $>5,>50$ and $>100 \mathrm{MeV})$ according to the GOES-11 data (https://www.swpc.noaa.gov/products/goes-proton-flux/); b) neutron monitor count rate in Calgary $\left(\Phi \sim 58^{\circ}\right.$, geomagnetic cutoff rigidity $\left.R_{c}=1.09 \mathrm{GV}\right)$ (ftp://ftp.ngdc.noaa.gov/STP/SOLAR_DATA/COSMIC_RAYS/STATION_DATA/Calgary);

c) daily number of X-ray solar flares (https://www.ngdc.noaa.gov/space-weather/solardata/solar-features/solar-flares/x-rays/goes/xrs/); d) daily mean ionization rate at geomagnetic latitudes 60-90 (https://solarisheppa.geomar.de/solarprotonfluxes).

\section{Variations of the polar vortex intensity during SPEs of January 2005}

As the polar vortex is seen in the stratosphere as a belt of enhanced western winds, let us use daily mean values of the U-component of wind velocity (directed from west to east) to characterize the vortex intensity. Fig. 2 demonstrates temporal variations of maximal values of wind velocity $\mathrm{U}_{\max }$ observed at different stratospheric levels in the region of the vortex formation in winter months 2004/2005 (the linear trends were removed), the data from the NCEP/NCAR reanalysis archive [Kalnay et al., 1996] being taken. One can see a noticeable strengthening of western winds in the course of the SPEs under study. The deviations from the trends amount to $\sim 20-30 \mathrm{~m} \cdot \mathrm{s}^{-1}$ at the upper stratospheric levels $30-10 \mathrm{hPa}$ (altitudes $\sim 25-35 \mathrm{~km}$ ) and $\sim 15 \mathrm{~m} \cdot \mathrm{s}^{-1}$ at the lower levels $100-50 \mathrm{hPa}(\sim 15-20 \mathrm{~km})$.

In Fig. 3 the charts of daily values of western wind velocity in the Northern hemisphere at the $50 \mathrm{hPa}$ level are presented for the period 13-21 January 2005. The polar vortex is seen as the belt of sharp enhancement of wind velocities at latitudes $50-80^{\circ} \mathrm{N}$. The area covered by western winds of high velocity $\left(\mathrm{U} \geq 40 \mathrm{~m} \cdot \mathrm{s}^{-1}\right)$ is shown in black. One can see that in the course of the studied SPEs this area was enlarging noticeably, with its longitudinal extension 
increasing. It should be also noted that before the event onset, this area was localized mostly over the Arctic coasts of North America, but in the course of the SPEs it extended to the North Atlantic and Scandinavia, with $U_{\max }$ values in it increasing from 55 to $65-67 \mathrm{~m} \cdot \mathrm{s}^{-1}$.
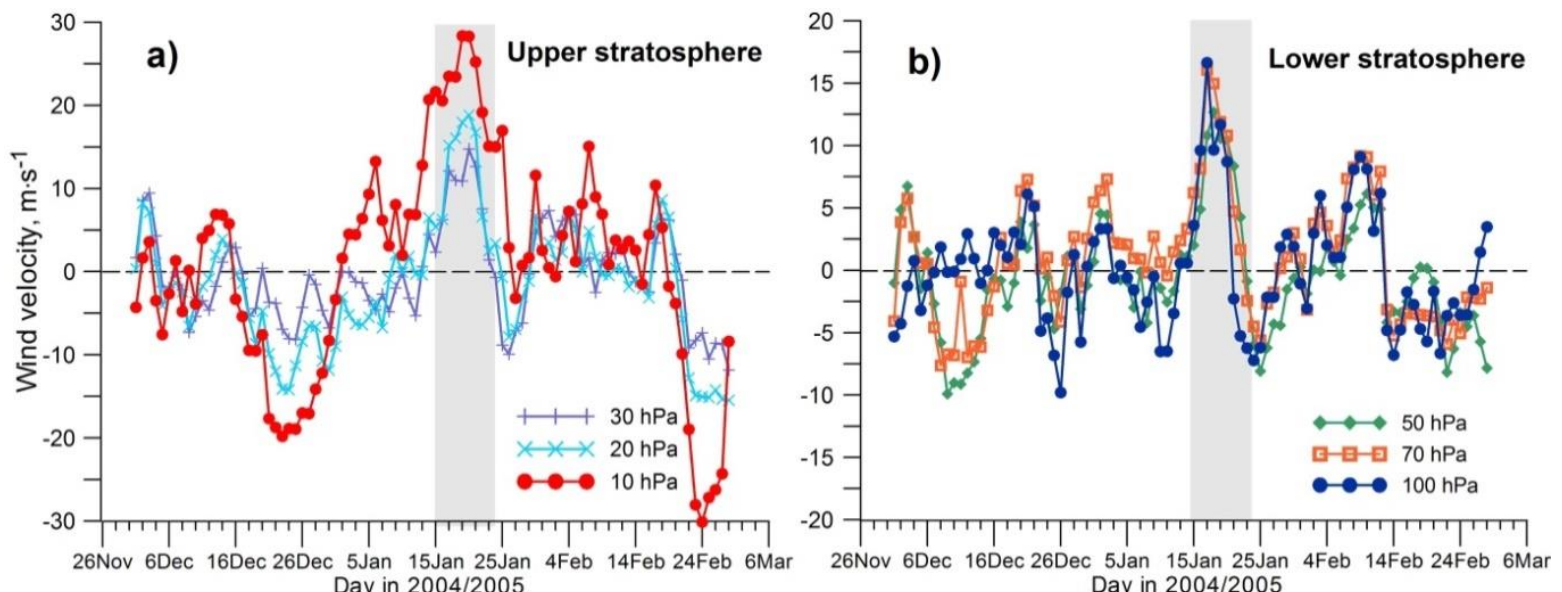

Fig.2. Temporal variations of maximal values (detrended) of western wind velocity $U_{\text {max }}$ in the upper (a) and lower (b) stratosphere. The period 15-23 January is shown with a gray background.
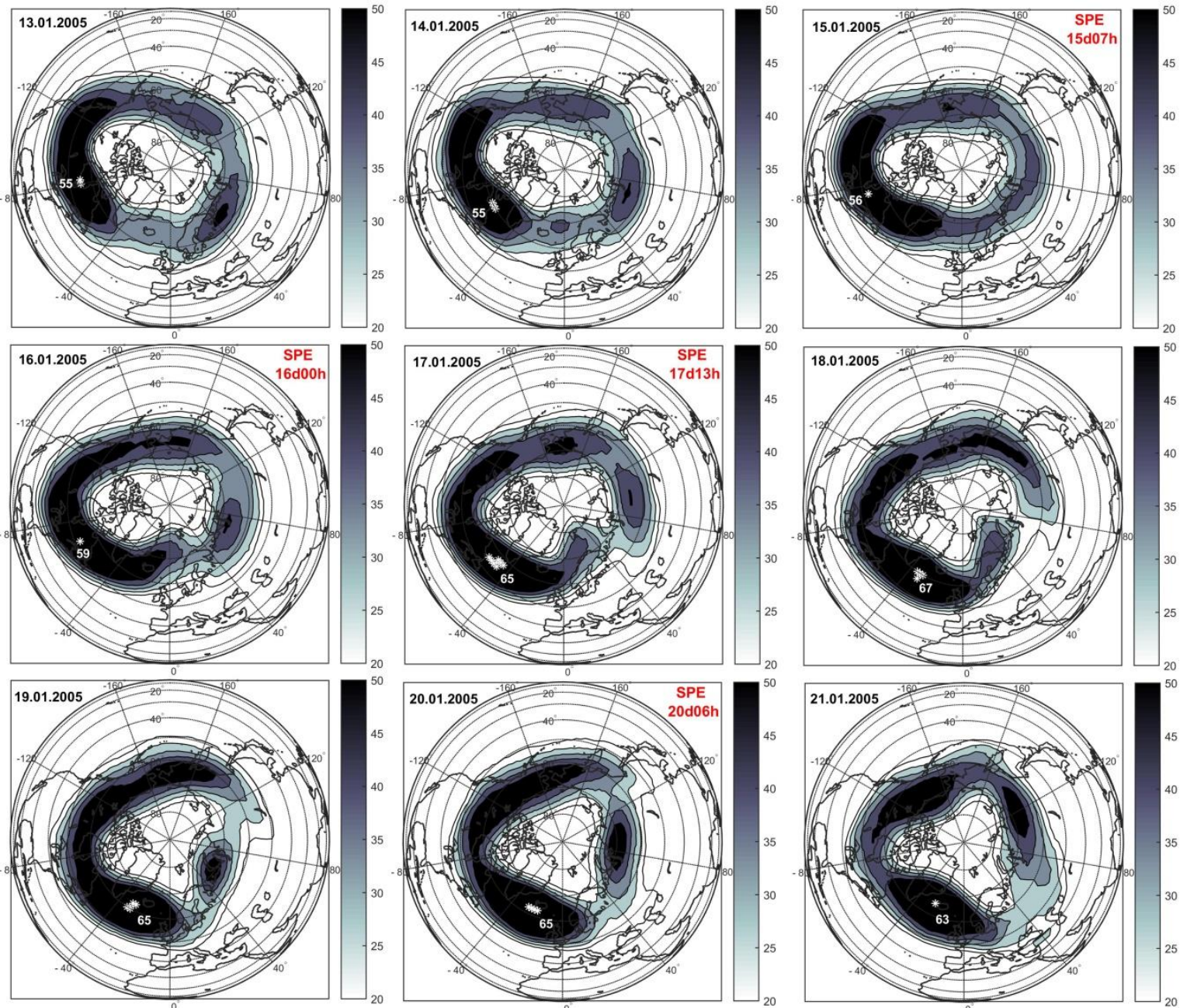

Fig.3. Charts of daily mean values of western wind velocity (in $\left.\mathrm{m} \cdot \mathrm{s}^{-1}\right)$ at the stratospheric level $50 \mathrm{hPa}$ for 13-21 January 2005. The areas of wind velocity $U \geq 40 \mathrm{~m} \cdot \mathrm{s}^{-1}$ are shown in black. Asterisks indicate maximal values of wind velocity. 
The enlargement of the areas of enhanced wind velocity was observed at all the stratospheric levels during the period 15-21 January 2005. Fig.4 demonstrates temporal variations of the areas (in fraction of the entire Earth's surface area) covered by western winds of high velocity (equal or exceeding some threshold value for each stratospheric level). One can see a noticeable enlargement of these areas associated with the SPEs under study (Fig.4a, b). Moreover, new areas of wind velocities which were not observed before the event onset appeared, e.g., the areas with $U \geq 100 \mathrm{~m} \cdot \mathrm{s}^{-1}$ at the $10 \mathrm{hPa}$ level and $\mathrm{U} \geq 60 \mathrm{~m} \cdot \mathrm{s}^{-1}$ at the $50 \mathrm{hPa}$ level (Fig.4c). Thus, the data presented in Figs.2, 3 and 4 provide evidence of a strong intensification of the stratospheric polar vortex during a series of SPEs of January 2005.
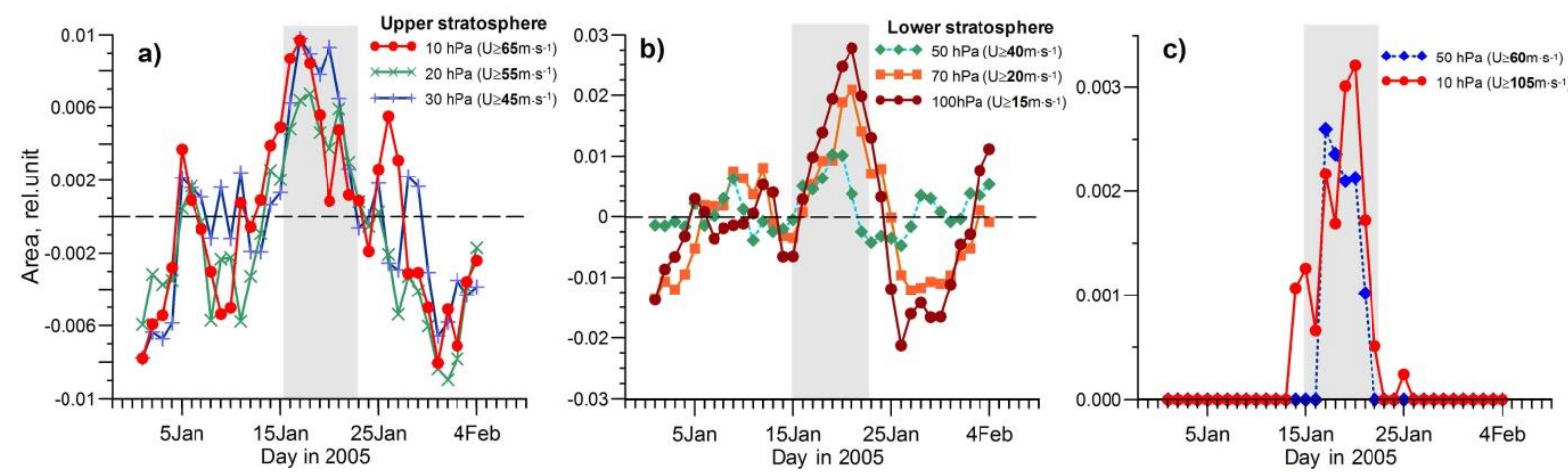

Fig.4. Temporal variations of the areas (in relative units) with enhanced wind velocity (threshold values are indicated in the legend) in the upper (a) and lower (b) stratosphere in January 2005; c) Temporal variations of the areas of extremely high (not observed before the SPE onset) velocities of western wind. The period 15-23 January is shown with a gray background.

\section{Possible factors of the polar vortex intensification}

Let us consider possible factors contributing to the vortex intensification. The vortex formation area is characterized by low geomagnetic cutoff rigidities allowing penetration of charged cosmic particles (cosmic rays) in a wide energy range. This provides higher values of ionization rates in this area compared with those at lower latitudes. Besides, the belt of enhanced wind velocities $60-80^{\circ} \mathrm{N}$ (Fig.3) turns out to be in a zone of maximal occurrence of auroral phenomena (auroral zone). Auroras are caused by precipitations of auroral electrons which lead to excitation of atmospheric constituents during magnetospheric substorms. Though auroral electron energy is absorbed mainly in the lower thermosphere (above $90 \mathrm{~km}$ ), they contribute to generation of bremsstrahlung X-rays which are capable to penetrate into the stratosphere. According to [Jackman, 1991], X-rays with energies $30 \mathrm{keV}$ and $10^{3} \mathrm{keV}$ may reach stratospheric altitudes, respectively, $\sim 40 \mathrm{~km}$ and $\sim 30 \mathrm{~km}$ producing ionization changes.

Temporal variations of maximal values of western wind velocity $U_{\max }$ at the upper stratospheric level $10 \mathrm{hPa}$ are compared with 5-day running averages of geomagnetic AE-index in Fig.5a. AE-index characterizes intensity of electric current (auroral electrojet) which develops in the ionosphere at high latitudes during magnetospheric substorms and is accompanied by precipitation of auroral electrons. One can note that temporal variations of maximal wind velocities $U_{\max }$ in the upper stratosphere and those of AE-index are very similar, with the maxima of $U_{\max }$ following the maxima of AE-index or coinciding with them. The correlation coefficient between $U_{\max }$ and AE-indices in winter months 2004/2005 amounts to $\sim 0.5$ and increases up to $\sim 0.7$ if AE-indices are smoothed over 5-day intervals.

Fig.5b shows a relationship between $\mathrm{U}_{\max }$ at the stratospheric level $10 \mathrm{hPa}$ in January 2005 and daily mean ionization rates in the upper stratosphere $(35 \mathrm{~km})$ at geomagnetic latitudes 60$90^{\circ}$ according to the SOLARIS-HEPPA data. It can be seen that maximal values of wind velocity varies in a wide range (from $\sim 80$ to $\sim 110 \mathrm{~m} \cdot \mathrm{s}^{-1}$ ) when ionization rate is less than $1 \mathrm{~cm}^{-}$ 
3. $\mathrm{s}^{-1}$. However, when ionization rate exceeds $1 \mathrm{~cm}^{-3} \cdot \mathrm{s}^{-1}$, wind velocity starts increasing (up to $\sim 120 \mathrm{~m} \cdot \mathrm{s}^{-1}$ ) with ionization increase. Thus, the increase of stratospheric ionization rate seems to contribute to the intensification of the polar vortex on the day-to-day time scale.
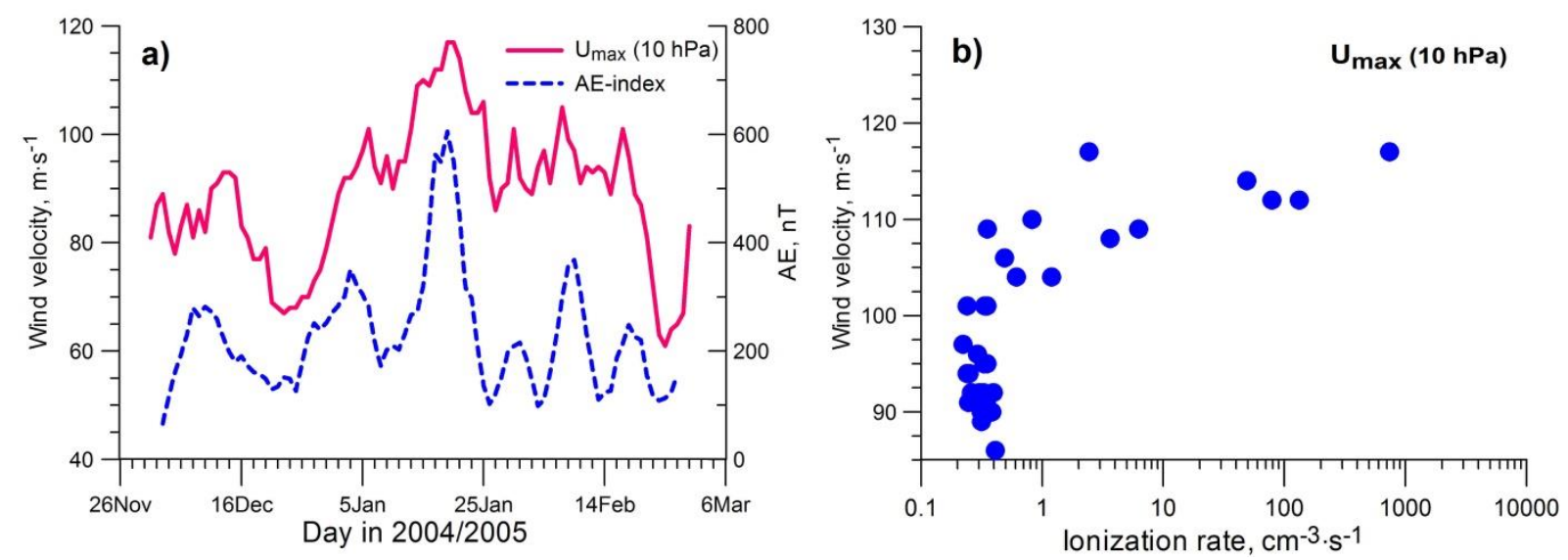

Fig.5. a) Temporal variations of maximal values of western wind velocity $U_{\text {max }}$ in the upper stratosphere (the $10 \mathrm{hPa}$ level) and 5-day running averages of geomagnetic AE-indices (ftp://ftp.ngdc.noaa.gov/STP/GEOMAGNETIC DATA/ INDICES/AURORAL ELECTROJET/) in winter months 2004/2005; b) maximal values of western wind velocity $U_{\max }($ the $10 \mathrm{hPa}$ level) in January 2005 versus daily mean ionization rates in the upper stratosphere (35 km).

The data above provide evidence for influence of phenomena associated with solar activity on the state of the stratospheric polar vortex on the day-to-day scale. An important part seems to be played by stratospheric ionization changes caused by solar proton events, the energy of particles being enough to penetrate into the stratosphere, as well as auroral activity (bremsstrahlung X-ray with energies above $30 \mathrm{keV}$ ). The obtained results suggest that pronounced increases of stratospheric ionization may contribute to the vortex intensification.

As wind velocity in the atmosphere is closely related to temperature gradients, the detected strengthening of the polar vortex indicates that a significant increase of temperature gradients between high and middle latitudes took place during the studied series of SPEs in January 2005. A possible reason for this increase may be changes of thermal-radiative balance of the highlatitudinal stratosphere. Indeed, increases of ionization caused by SPEs lead to noticeable changes of chemical composition of the polar atmosphere, in particular, to enhanced production of nitrogen and hydrogen oxides, which participate in catalytic destruction of ozone (e.g., [Krivolutsky and Repnev, 2009]). As ozone can influence significantly fluxes both of shortwave and longwave radiation, a reduction of its content may result in some changes of temperature regime of the polar atmosphere, namely radiative cooling of the atmosphere under polar night conditions, which contribute to an increase of temperature gradients between polar and middle latitudes and, then, a strengthening of the polar vortex. Thus, the detected effects of strong SPEs on the polar vortex intensity may be related to changes of chemical composition of the polar atmosphere caused by SPE-induced ionization changes and, in turn, influencing its temperature regime.

\section{Conclusions}

1. Intensity of the stratospheric polar vortex may be affected noticeably by phenomena related to solar/geomagnetic activity on the day-to-day time scale.

2. An appreciable strengthening of the polar vortex (an increase of western wind velocity in the latitudinal belt $60-80^{\circ} \mathrm{N}$ ) was revealed at all the stratospheric levels in the course of Solar Proton Events in January 2005, with energies of particles being enough to reach stratospheric heights. 
3. Auroral activity (auroral electron precipitations generating bremsstrahlung X-rays) may be an additional factor contributing to the polar vortex intensification.

4. A possible reason for the polar vortex intensification may be changes of temperature regime of the polar atmosphere resulting from changes of its chemical composition caused by intrusion of energetic solar protons.

\section{References}

Baldwin, M.P., Dunkerton, T.J. (2001) Stratospheric harbingers of anomalous weather regimes. Science, 294, 581584.

Jackman, C.H. (1991) Effects of energetic particles on minor constituents of the middle atmosphere. J. Geomag. Geoelectr., 43, Suppl., 637-646.

Kalnay, E., Kanamitsu, M., Kistler, R. et al. (1996) The NCEP/NCAR 40-year reanalysis project. Bull. Amer. Meteorol. Soc., 77, 437-472.

Krivolutsky, A.A., Repnev, A.I. (2009) Cosmic influences of the ozonosphere of the Earth. Moscow, GEOS, $384 \mathrm{p}$.

Tinsley, B.A. (2008) The global atmospheric electric circuit and its effects on cloud microphysics. Reports on Progress in Physics, 71 (6), 66801-66900.

Tinsley, B.A. (2012) A working hypothesis for connections between electrically-induced changes in cloud microphysics and storm vorticity, with possible effects on circulation. Adv. Space Res., 50, 791-805.

Veretenenko, S., Ogurtsov M., (2019) Manifestation and possible reasons of $\sim 60$-year oscillations in solaratmospheric links. Adv. Space Res., 64 (1), 104-116. doi: 10.1016/j.asr.2019.03.022. 


\title{
The GIC growth in electric power lines during magnetic storm on 7-8 September 2017
}

\author{
Belakhovsky V.B. ${ }^{1}$, Pilipenko V.A. ${ }^{2}$, Sakharov Ya.A. ${ }^{1}$, Selivanov V.N. ${ }^{3}$ \\ ${ }^{1}$ Polar Geophysical Institute, Apatity \\ ${ }^{2}$ Institute of the Physics of the Earth, Moscow \\ ${ }^{3}$ Kola Scientific Center RAS, Apatity
}

\begin{abstract}
It is considered the growth of the geomagnetically induced currents (GIC) in electric power lines at Kola Peninsula and Karelia (Russia) during strong geomagnetic storm 7-8 September 2017. We used the GIC registration system in electric power lines created by the Polar Geophysical Institute and Kola Scientific Center. The IMAGE magnetometer data was used for the analyses of the geomagnetic field variability. The $\mathrm{dY} / \mathrm{dt}$ has comparable contribution to the GIC growth as dX/dt. So these GIC are dangerous for the north-south oriented technological systems. There are two intervals of the strong GIC growth (more than $50 \mathrm{~A})$ was registered during the storm. It is found that one interval the significant contribution have the vortex-like ionosphere current systems, for the another time interval auroral electrojet have dominant contribution in comparison with vortex current systems. The fine structure of the substorm (Pi3 pulsations) produce vortex-like current systems connected with the fieldaligned currents in the magnetosphere.
\end{abstract}

\section{Introduction}

One of the most significant factors of space weather for terrestrial technological systems is geomagnetically induced currents (GICs) in conductor systems caused by abrupt changes of the geomagnetic field [Lanzerotti, 2001]. GICs associated with great magnetic disturbances were found to be dangerous for various technological systems, causing malfunction of railway equipment, disruption of ground and transatlantic communication cables, deleterious impacts on telephone lines, and reduction of the lifetime of pipelines [Pirjola et al. 2005].

GIC are often modeled as fluctuations of intensity of the East-West auroral electrojet producing telluric currents in the longitudinal direction [Boteler et al. 1998]. On the basis of these notions, it is commonly supposed that geomagnetic disturbances are most dangerous for technological systems (like power lines, and oil/gas pipe lines) extended in the longitudinal direction. However, it was found that fast small-scale ionospheric current structures can provide a significant contribution to rapid geomagnetic field variations, responsible for GIC generation [Viljanen et al. 2001; Belakhovsky et al., 2018; Belakhovsky et al., 2017]. Thus, to characterize the geomagnetic field variability one needs finer characteristics than the widely used time derivative of the $X$-component (North-South) of the geomagnetic field $\mathrm{d} X / \mathrm{dt}$ [Belakhovsky et al., 2019]. It is still tempting to find an adequate tool to reveal the temporal-spatial features of geomagnetic field variations most relevant to the GIC generation.

[Dimmock et al., 2019] examined the impact of this storm on GIC in a gas pipeline in southern Finland. Although this storm was moderate as judged by the global Dst index ( - 150 nT), it led to an unexpectedly large GIC burst (> $10 \mathrm{~A})$, which had not previously been observed in this system even under large geomagnetic disturbances.

Here we consider the contribution of geomagnetic disturbances to the rapid growth of the GIC in electric power lines of Kola Peninsula and Karelia for the 7-8 September 2017 strong geomagnetic storm. 


\section{Data and methods}

A system to monitor the impact of GIC on power lines was deployed in 2011 in the Kola Peninsula and Karelia by the Polar Geophysical Institute and the Center for Physical and Technical Problems of North's Energetic. The system consists of 4 stations at $330 \mathrm{kV}$ power line and a station at the $110 \mathrm{kV}$ power line. Each station records a quasi-DC current in the deadgrounded neutral of the transformer.

The variations of the geomagnetic field were measured by IMAGE magnetometers with 10 -sec time resolution. For an array of magnetometers oriented along a geomagnetic meridian, the vector diagram method can be applied. The Finnish Meteorological Institute provides the online (http://space.fmi.fi/image/beta/) capability to compute and visualize 2D ionospheric equivalent current vectors from the IMAGE magnetometers. For the equivalent current modeling, the method of spherical elementary current systems has been used [Amm \& Viljanen, 1999]. The technique determines the divergence-free component of the equivalent ionospheric currents (which roughly describes the distribution of ionospheric Hall currents) from groundbased magnetometer data.

\section{GIC growth during magnetic storm on 7-8 September 2017}

We consider the strong magnetic storm on 7-8 September 2017 that was initiated by an interplanetary shock arrival at $\sim 23: 30$ UT on 7 September. After $~ 20: 00$ UT on 7 September, IMF Bz gradually turned southward $(<0)$ and remained steady at about $-10 \mathrm{nT}$ to $-30 \mathrm{nT}$ until $\sim 04: 00$ UT on 8 September. The solar wind speed reach the high value $870 \mathrm{~km} / \mathrm{s}$. This produced driving of the magnetosphere into a magnetic storm, during which geomagnetic indices reached maximal values of $|\mathrm{Dst}| \sim 150 \mathrm{nT}$ and $\mathrm{AE} 2700 \mathrm{nT}$.

This period coincided with a period of maximum of magnetic bay magnitude at the IMAGE magnetic stations from 20-04 UT (Fig. 1). The magnetic bay was observed only in the $X$ component (more than $3000 \mathrm{nT}$ at SOD station).
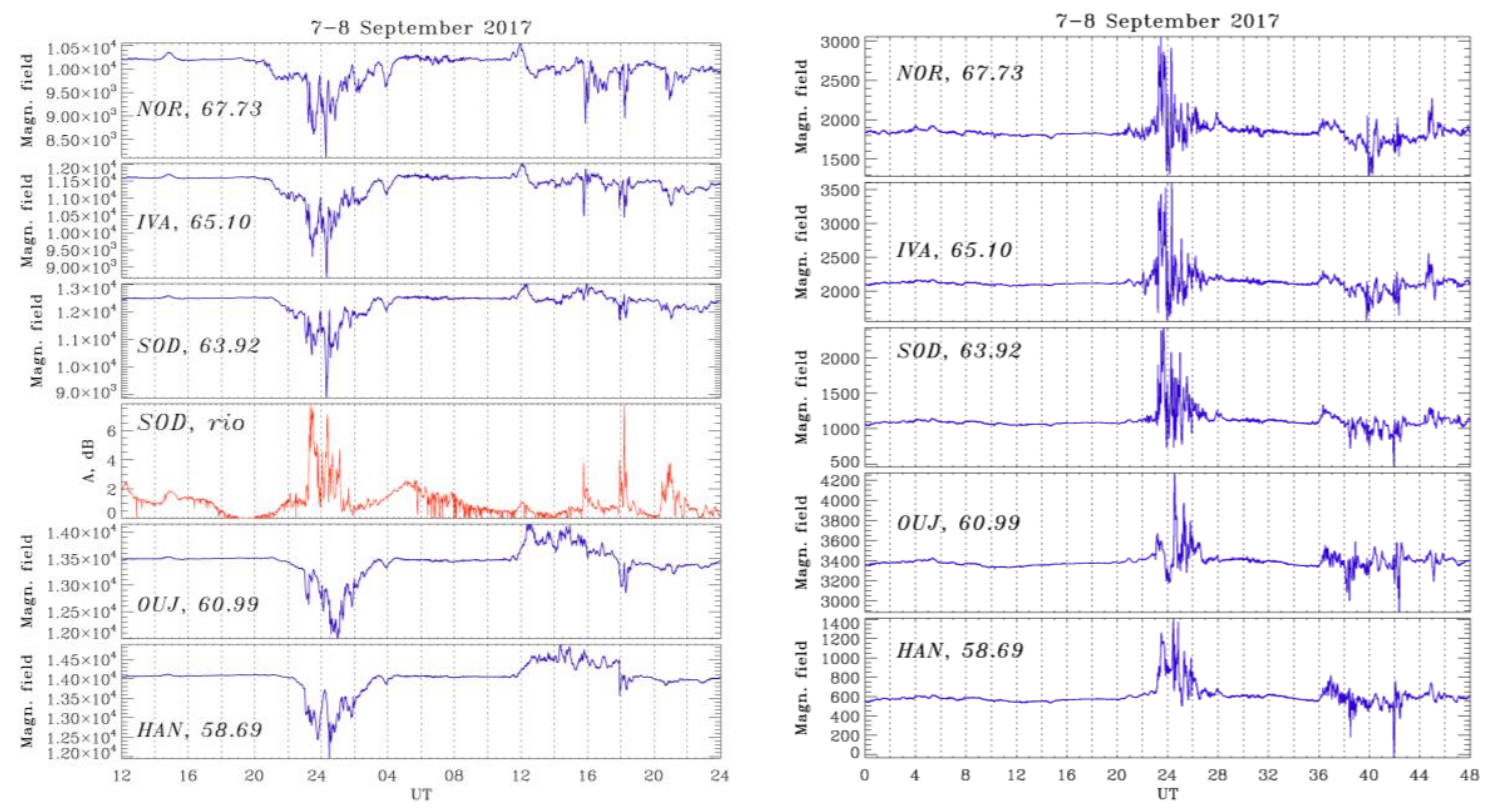

Fig. 1. X-component of geomagnetic fields at the latitudinal array of stations NOR-IVA-SOD-OUJ-

$H A N$ and the cosmic noise absorption at SOD riometer station [dB] (left panel), the $Y$ component of the geomagnetic field(right panel) for the 7-8 September 2017. Geomagnetic latitudes are indicated near the station codes.

During the period of maximal magnetic disturbance, intense Pi3 pulsations were superposed on the magnetic bay. These pulsations are not quasi-sinusoidal waves like typical Pc5 
pulsations; they are rather quasi-periodic sequences of magnetic impulses. The time scale of these oscillations varies from $\sim 20 \mathrm{~min}$ at lower latitudes up to $\sim 10 \mathrm{~min}$ at higher latitudes (Fig. 1).

There are two peaks of the significant GIC growth (more than $50 \mathrm{~A}$ ) was observed during this magnetic storm. During first interval extremely high values of GIC were recorded (up to $\sim 85$ A per node) at station VKH, from 22:00 to 02:00 UT on 7-8 Sept 2013 (Fig. 2). At lower latitude KND station the value of the GIC was about $15 \mathrm{~A}$. The data for another GIC stations was not available for this event. The second peak of GIC was observed at 20 UT on 8 September 2017, it was short time increase (5-10 minutes).

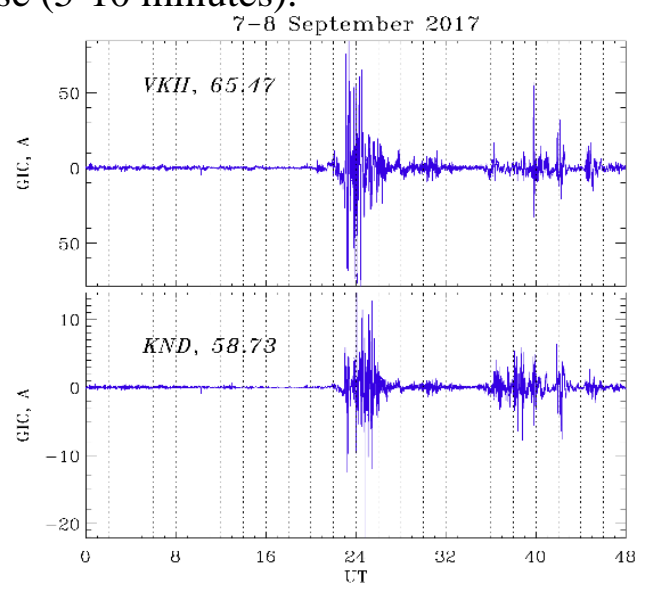

Fig. 2. GIC data at stations VKH, KND for the 7-8 September 2017. Geomagnetic coordinates are shown near station codes.

The substorm at 23-02 UT was accompanied by the strong increase of the cosmic noise absorption (CNA) with amplitude more than $7 \mathrm{~dB}$ as seen from the SOD riometer, the second disturbance was accompanied by the growth of the CNA $(>7 \mathrm{~dB})$ as well. It testifies about the presence of the powerful electron precipitations into the ionosphere.

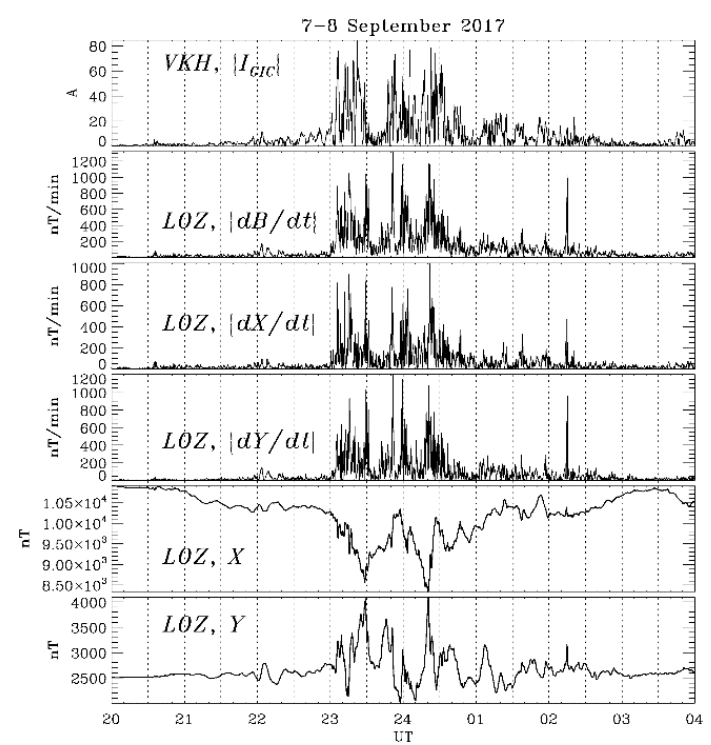

Fig. 3. Comparison between GIC amplitudes, time derivatives $|d X / d t|$ and $|d Y / d t|$ [nT/min], and $\Delta X$ and $\triangle Y$ components of geomagnetic field $\left[10^{4} \cdot n T\right]$ at nearby stations VKH and LOZ for the 7-8 September 2017.

During the magnetic storm the magnetic disturbance gradually increased and then slowly decayed, and was mainly oriented in the X-direction. However, during the maximal disturbance magnetic variations became more chaotic. Comparison of the magnitude of magnetic disturbances $\Delta \mathrm{X}$ and $\Delta \mathrm{Y}$ with amplitudes of time derivatives $|\mathrm{d} X / \mathrm{dt}|,|\mathrm{d} Y / \mathrm{dt}|$ (Fig. 3.) shows that 
though the magnetic disturbance was much larger in the $\mathrm{X}$-component than in the $\mathrm{Y}$-component, $|\Delta X|>>|\Delta Y|$, the time derivative $|\mathrm{d} Y / \mathrm{dt}| \geq 1200 \mathrm{nT} / \mathrm{min}$ was larger than the time derivative $|\mathrm{d} X / \mathrm{dt}| \geq 1000 \mathrm{nT} / \mathrm{min}$. Therefore, variations of both horizontal components provided a similar contribution to the increase of $|\mathrm{d} \mathbf{B} / \mathrm{dt}|$. Magnetic field variations are composed from time variations and variations caused by fast azimuthal drift of Pi3 structures.

To present the picture of the dynamics of ionospheric currents along the meridional profile, geomagnetic disturbances $\mathrm{B}=\{\mathrm{X}, \mathrm{Y}\}$ for each station of the profile were drawn on one graph in the form of sequentially time-shifted disturbance vectors. The perturbation of the magnetic field $\mathrm{B}$ at the observation point is associated with the equivalent ionospheric current $\mathrm{J}$ above it as follows $B=(2 \pi / c)[J \times n]$, where $n$ is the normal to the plane. Vector $J$ is rotated by $\pi / 2$ clockwise with respect to $\mathrm{B}$ (but its value will be indicated in $\mathrm{nT}$ ).

The vector diagrams of the equivalent ionospheric current variations (Fig. 4.) with time cadence 1 min show that the Pi3 pulsations were a sequence of localized vortex-like structures for the interval 15-22 UT. But for the interval 20-04 UT it is seen the dominant contribution of the auroral electrojet.
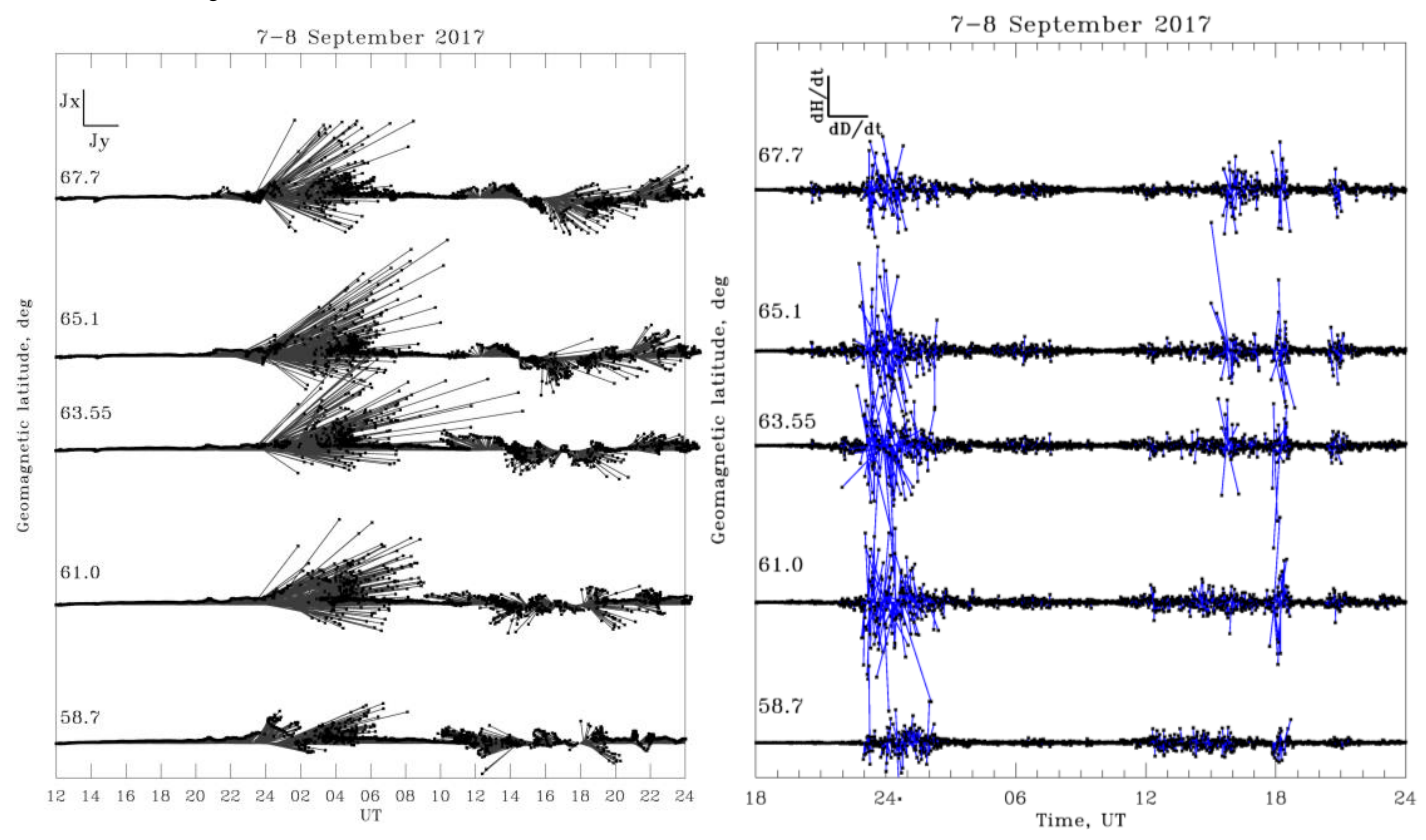

Fig. 4. Vector diagrams of equivalent ionosphere currents (left panel) and the vector diagram of the $d B / d t$ (right panel) for the 7-8 September 2017 with time cadence 1 min.

The spherical elementary current method (SECS) [Amm and Viljanen, 1999] is used to calculate the field of equivalent currents. The method is based on the division of horizontal currents integrated over the height of the ionosphere into vortex (divergence-free) and potential components. Horizontal irregular currents close the field-aligned currents connecting the upper atmosphere with magnetospheric processes. From the ground data, a divergence-free (vortex) component is calculated, which approximately describes the distribution of the ionospheric Hall currents. These method of 2D equivalent currents reveals the formation of the vortex-like intensifications during the growth of GIC with epicenter at $66^{\circ}-67^{\circ}$ geomagnetic latitudes, i.e. under the Kola Peninsula (Fig. 5.). It is seen that for some moments the ionosphere currents have vortex-like structure (left panel), for another moment the structure of the ionosphere currents is complicate. It seems like a mixture of the auroral electrojet and vortex like currents connected with the field-aligned currents in the magnetosphere. 

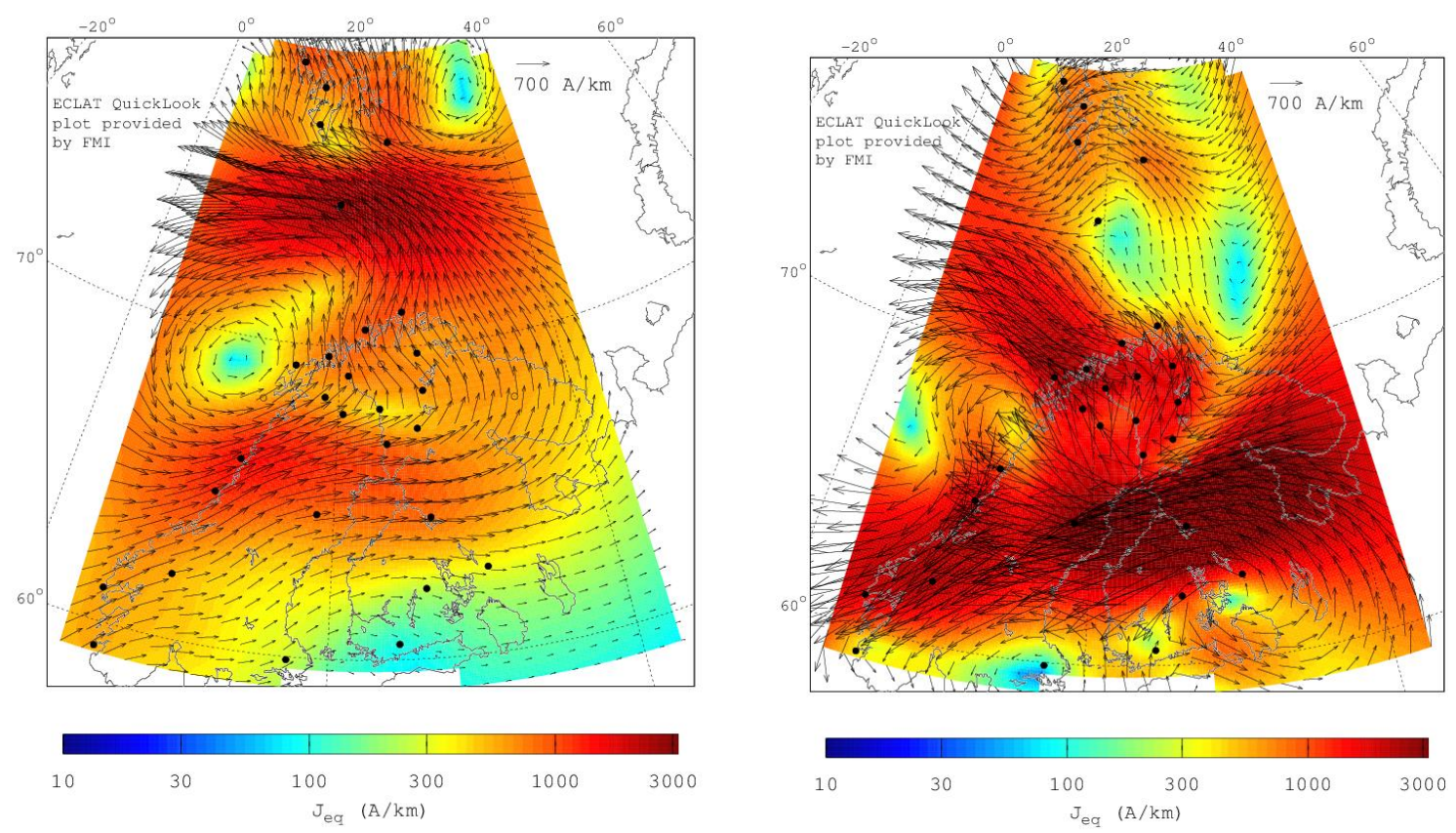

Fig. 5. The 2D model of equivalent ionosphere currents constructed from the IMAGE magnetometer data for the 7-8 September 2017 at 16.05 UT (left) and 23.57 UT (right).

A comparison of the instantaneous amplitudes of the GIC at VKH and KND stations with the "magnetic keogram", showing the time variation of the latitudinal distribution along the profile of the IMAGE stations of the amplitude of the geomagnetic disturbance (X-component) and the field variability $\mathrm{dX} / \mathrm{dt}$, is presented in Fig. 6. Six activations are accompanied by a burst of $\mathrm{dB} / \mathrm{dt}$ and GIC. Disturbances of the magnetic field occur at latitudes $58^{\circ}-67^{\circ}$. For all activations, a general tendency is visible - the disturbance first arises at high latitudes, and then spreads to lower ones.

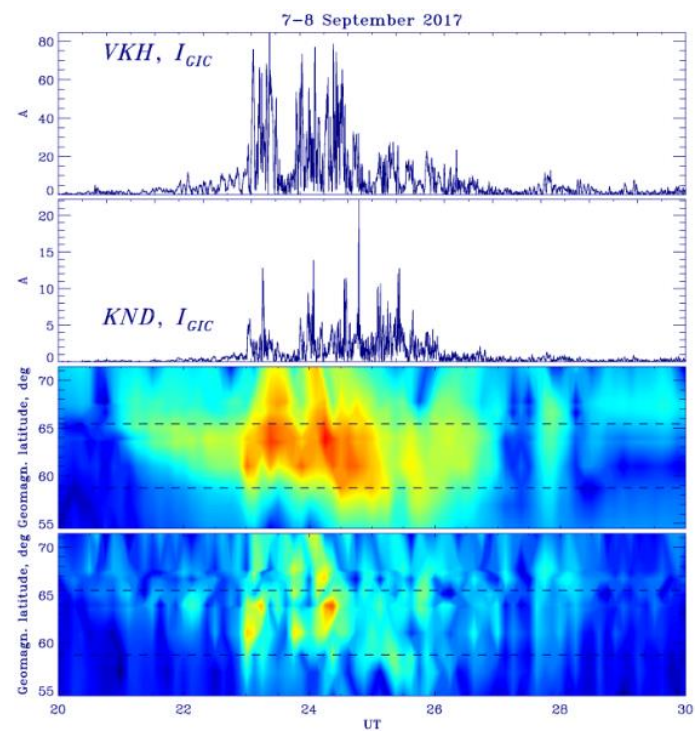

Fig. 6. The GIC at VKH, KND stations; latitude-UT distribution of the geomagnetic field variations (X-component) according to the IMAGE magnetometer data; latitude-UT distribution of the $d X / d t$. 
The epicenter of the field variability $\mathrm{dX} / \mathrm{dt}$ localized in latitude in the range of $60^{\circ}-65^{\circ}$. The transmission lines from VKH to KND cover an overlapping area of latitudes, from $62^{\circ}$ to $68^{\circ}$. Thus, during this magnetic storm, the "epicenter" of the geomagnetic disturbance was within the area covered by the power transmission line. The analysis of the spatial-temporal distribution of the geomagnetic field variations are does not coincide with the spatial-temporal distribution of its derivation during the strong GIC growth at VKH and KND stations. So the strong GIC is not always associated with the strong geomagnetic disturbance but it associated with fast geomagnetic disturbances embedded into strong magnetic bay.

\section{Conclusions}

The analysis of the geomagnetic disturbances and GIC variations for the strong magnetic storm 7-8 September 2017 shows that the large-scale structure of the X-component of the disturbed geomagnetic field is mainly determined by the ionospheric East-West electrojet. In smaller regional scales, weaker but rapidly varying localized vortex-like current systems are superposed on the electrojet. These current structures produce intense GICs, as observed by the GIC recording system of the power lines in the Kola Peninsula.

A quasi-periodic sequence of localized vortex-like structures observed by magnetometers produces very high values of GIC (up to $\sim 85 \mathrm{~A}$ ). The night-side solitary vortices observed as magnetic pulses with large amplitudes superposed on the substorm-related magnetic bay have been observed to be accompanied by very intense GICs. These results have confirmed that GIC cannot be modeled by a simple model of the extended ionospheric current and dictate the necessity to take into account superposed localized vortex-like current systems.

\section{Reference}

Amm O., A. Viljanen. Ionospheric disturbance magnetic field continuation from the ground to the ionosphere using spherical elementary current systems // Earth Planets Space, 51, 431-440, 1999.

Belakhovsky V.B., V.A. Pilipenko, Ya.A. Sakharov, D.L. Lorentzen, S.N. Samsonov. Geomagnetic and ionospheric response to the interplanetary shock on January 24, 2012 // Earth, Planets and Space, 69:105, doi:10.1186/s40623-017-0696-1, 2017.

Belakhovsky V.B., V.A. Pilipenko, Ya.A. Sakharov, V.N. Selivanov. Characteristics of the geomagnetic field variability for the study of the magnetic storm and substorm impact on electrical power systems // Physics of Solid State, №1, 173-185, 2018.

Belakhovsky V., Pilipenko V., Engebretson M., Sakharov Ya. and Selivanov V. Impulsive disturbances of the geomagnetic field as a cause of induced currents of electric power lines // Journal of Space Weather and Space Climate. 9. A18. 2019

Boteler D.H., R.J. Pirjola, H. Nevanlinna. The effects of geomagnetic disturbances on electrical systems at the Earth's surface // Adv. Space Res. 22, 17-27, 1998.

Dimmock A.P., Rosenqvist L., Hall J.-O., Viljanen A., Yordanova E., Honkonen I., André M., Sjöberg E.C. The GIC and geomagnetic response over Fennoscandia to the 7-8 September 2017 geomagnetic storm // Space Weather, 17. 2019.

Lanzerotti L.J. Space weather effects on technologies // Space Weather, Geophys. Monogr. Ser. AGU. 125, p. 11, 2001.

Pirjola R., K. Kauristie, H. Lappalainen, A. Viljanen, A. Pulkkinen. Space weather risk // Space Weather. 3. S02A02. 2005.

Viljanen A., H. Nevanlinna, K. Pajunpaa, A. Pulkkinen. Time derivative of the geomagnetic field as an activity indicator. Ann. Geophys. 19. 1107-1118. 2001.

Acknowledgements. We thank the national institutes that support the IMAGE magnetic observatories (http://www.ava.fmi.fi/image). The interplanetary parameters were taken from the OMNI database (https://omniweb.gsfc.nasa.gov). We thank to the Sodankyla Geophysical Observatory (https://www.sgo.fi) for the possibility to use riometer data. Data from the PGI system are available at EURISGIC (European Risk for Geomagnetically Induced Currents) website http://eurisgic.org. 